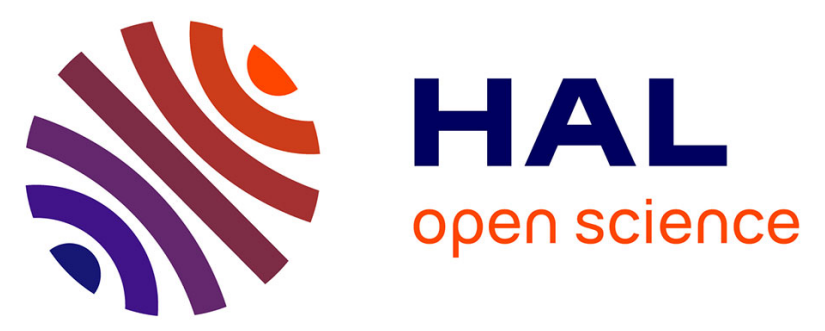

\title{
Novel IQCE variations confirm its role in postaxial polydactyly and cause ciliary defect phenotype in zebrafish
}

Alejandro Estrada-Cuzcano, Christelle Etard, Clarisse Delvallée, Corinne Stoetzel, Elise Schaefer, Sophie Scheidecker, Véronique Geoffroy, Aline

Schneider, Fouzia Studer, Francesca Mattioli, et al.

\section{To cite this version:}

Alejandro Estrada-Cuzcano, Christelle Etard, Clarisse Delvallée, Corinne Stoetzel, Elise Schaefer, et al. Novel IQCE variations confirm its role in postaxial polydactyly and cause ciliary defect phenotype in zebrafish. Human Mutation, 2019, 10.1002/humu.23924 . hal-02304111

\author{
HAL Id: hal-02304111 \\ https://hal.science/hal-02304111
}

Submitted on 2 Oct 2019

HAL is a multi-disciplinary open access archive for the deposit and dissemination of scientific research documents, whether they are published or not. The documents may come from teaching and research institutions in France or abroad, or from public or private research centers.
L'archive ouverte pluridisciplinaire HAL, est destinée au dépôt et à la diffusion de documents scientifiques de niveau recherche, publiés ou non, émanant des établissements d'enseignement et de recherche français ou étrangers, des laboratoires publics ou privés. 


\section{Novel IQCE variations confirm its role in postaxial polydactyly and cause ciliary defect phenotype in zebrafish.}

Alejandro Estrada-Cuzcano ${ }^{1 *}$, Christelle Etard ${ }^{2 *}$, Clarisse Delvallée ${ }^{1 *}$, Corinne Stoetzel ${ }^{1}$, Elise Schaefer ${ }^{1,3}$, Sophie Scheidecker ${ }^{1,4}$, Véronique Geoffroy ${ }^{1}$, Aline Schneider ${ }^{1}$, Fouzia Studer ${ }^{5}$, Francesca Mattioli ${ }^{6}$, Kirsley Chennen ${ }^{1,7}$, Sabine Sigaudy ${ }^{8}$, Damien Plassard ${ }^{9}$, Olivier Poch ${ }^{7}$, Amélie Piton ${ }^{4,6}$, Uwe Strahle ${ }^{2}$, Jean Muller ${ }^{1,4 *}$ and Hélène Dollfus ${ }^{1,3,5 *}$

1. Laboratoire de Génétique médicale, UMR_S INSERM U1112, IGMA, Faculté de Médecine FMTS, Université de Strasbourg, Strasbourg, France.

2. Karlsruhe Institute of Technology (KIT), Institute of Toxicology and Genetics (ITG), Eggenstein-Leopoldshafen, Germany.

3. Service de Génétique Médicale, Institut de Génétique Médicale d'Alsace, Hôpitaux Universitaires de Strasbourg, Strasbourg, France.

4. Laboratoires de Diagnostic Génétique, Hôpitaux Universitaires de Strasbourg, Strasbourg, France.

5. Centre de Référence pour les affections rares en génétique ophtalmologique, CARGO, Filière SENSGENE, Hôpitaux Universitaires de Strasbourg, 67091 Strasbourg, France.

6. Institut de Génétique et de Biologie Moléculaire et Cellulaire, 67400 Illkirch-Graffenstaden, France; INSERM U1258, 67400 Illkirch-Graffenstaden, France; CNRS UMR 7104, 67400 Illkirch-Graffenstaden, France; Université de Strasbourg, 67400 Illkirch, France.

7. Complex Systems and Translational Bioinformatics, ICube UMR 7357, Université de Strasbourg, Fédération de Médecine Translationnelle, Strasbourg, France.

8. Département de Génétique Médicale, Hôpital de la Timone, Marseille, France.

9. Plateforme GenomEast, IGBMC, Illkirch, France.

*equal contributor

Corresponding Author: Jean Muller/Hélène Dollfus

Email address: jeanmuller@unistra.fr / dollfus@unistra.fr 


\section{ABSTRACT}

Polydactyly is one of the most frequent inherited defects of the limbs characterized by supernumerary digits and high genetic heterogeneity. Among the many genes involved, either in isolated or syndromic forms, 8 have been implicated in postaxial polydactyly (PAP). Among those IQCE has been recently identified in a single consanguineous family. Using whole-exome sequencing in patients with uncharacterized ciliopathies including PAP, we identified 3 families with biallelic pathogenic variations in IQCE. Interestingly, the c.895_904del (p.Val301Serfs*8) was found in all families without sharing a common haplotype, suggesting a recurrent mechanism. Moreover, in 2 families, the systemic phenotype could be explained by additional pathogenic variants in known genes (TULP1, ATP6V1B1). RNA expression analysis on patients' fibroblasts confirms that dysfunction of IQCE leads to dysregulation of genes associated with the hedgehogsignaling pathway and zebrafish experiments demonstrate a full spectrum of phenotypes linked to defective cilia: body curvature, kidney cysts, left right asymmetry, misdirected cilia in pronephric duct and retinal defects. In conclusion, we identified 3 additional families confirming IQCE as a non-syndromic PAP gene. Our data emphasize, the importance of taking into account the complete set of variations of each individual as each clinical presentation could finally be explained by multiple genes.

Keywords: IQCE, cilia, polydactyly, hedgehog signaling, zebrafish, RNA-seq 


\section{INTRODUCTION}

Polydactyly is one of the most frequent inherited defects of the limb and has a prevalence of 0.33.6/1000 in live-births (Castilla et al., 1973; Malik, Ullah, Afzal, Lal, \& Haque, 2014). It is characterized by supernumerary digits, ranging from extra additive soft tissue without bone structure to an integral and fully developed digit (Umair, Ahmad, Bilal, Ahmad, \& Alfadhel, 2018). According to the position of the extra digit, polydactyly has been classified into postaxial (PAP), mesoaxial and preaxial (Malik, 2014). This defect is also associated with at least 221 syndromes according to the London Dysmorphology Database (Phadke \& Sankar, 2010) covering 146 genes (Umair et al., 2018) and can be a hallmark for a group of syndromes such as for ciliopathies. For this group of diseases, in addition to skeletal malformations such as PAP, the phenotypic spectrum can associate retinal degeneration, obesity, kidney dysfunction and sometimes intellectual disability as observed in the classical Bardet-Biedl syndrome (BBS; MIM\# 209900) for which 22 genes have been identified. In such heterogeneous diseases, high throughput genetic testing has made possible the diagnosis for many individuals (Shaheen et al., 2016) but also revealed more complex cases. Indeed, multi loci bearing pathogenic variations have been identified in several known disease related loci in single patients with apparent syndromic presentation (Karaca et al., 2018).

To date, eight genes FAM92A1, GLI1, GLI3, IQCE, KIAA0825, MIPOL1, PITX1 and ZNF141 (Ullah et al., 2019; Umair et al., 2018) have been implicated in non-syndromic polydactyly accounting for 286 disease causing variations according to HGMD (2018.1). Among those genes, IQCE (IQ domain-containing protein E) encodes a ciliary protein located at the base of the primary cilia and is linked to the Hedgehog $(\mathrm{Hh})$ signaling pathway (Pusapati et al., 2014). Hh signaling is one of many major pathways that controls key steps of embryonic development (Ingham, Nakano, \& Seger, 2011), tissue homeostasis and regeneration (Petrova \& Joyner, 2014) and related to human cancers (Barakat, Humke, \& Scott, 2010). Human hand and feet development is based on 
a highly conserved pentadactyl pattern (Abbasi, 2011), the conserved Hh signaling pathway regulates the precise digital shape and pattern in the limbs. IQCE together with EFCAB7 have an important regulatory role in the EvC complex (EVC-EVC2), a positive tissue regulator of $\mathrm{Hh}$ signaling. Recently, a homozygous splice acceptor variation in IQCE has been described in a single consanguineous family with PAP (Umair et al., 2017).

In our study, we report three families with biallelic pathogenic variations in IQCE identified by whole exome sequencing. Interestingly, these families with PAP were initially recruited as syndromic ciliopathies and two have additional pathogenic variations in other genes explaining their apparent syndromic phenotype. Functional studies based on the patient's cells or zebrafish (Danio rerio) assays confirm the ciliary role of IQCE.

\section{MATERIALS AND METHODS}

\section{Subjects}

The patients were recruited by the Strasbourg University Hospital Medical Genetics Department as well as by the Center for Rare Genetic Ophthalmologic Diseases (CARGO, Hôpitaux Universitaires de Strasbourg, Strasbourg) because they were suspected to have a ciliopathy. Study protocols used in each cohort have been approved by the corresponding Institutional Review Board or equivalent committees (in Strasbourg, "Comité de Protection des Personnes" EST IV, NDC-20142222), and written informed consent was given by each participant or parents. Our research complies with the Declaration of Helsinki. Written informed consent for open-access publication was provided by the participants or their parents. 


\section{Whole-exome sequencing}

Whole exome sequencing was performed by IntegraGen (Evry, France) for family A, B and C in 2011, 2013 and 2018 respectively. After shearing of the genomic DNA, library preparation was performed using either the Agilent Human All Exon Kits v2 (family A and B) or the Twist Human Core Exome Enrichment System (Twist Bioscience) and IntegraGen Custom additions (family C). Sequencing was performed on an Illumina HiSeq 2000/4000 to generate 75-bp paired-end reads following the manufacturer's protocols. Image analysis and base calling were performed using Illumina Real Time Analysis (1.14/2.7.7) with default parameters.

\section{Bioinformatics analysis}

The reads were mapped to the reference human genome (GRCh37/hg19) using BWA v0.7.12 (Etard, Roostalu, \& Strahle, 2010). GATK UG v3.4-46 was used to call SNV and indel variations (DePristo et al., 2011). Annotation and ranking of SNV and indel were performed by VaRank 1.4.3 (Geoffroy et al., 2015) in combination with the Alamut Batch software (1.11, Interactive Biosoftware, Rouen, France). Very stringent filtering criteria were applied to filter out nonpathogenic variants (Supp. Table 1): (i) variants represented with an allele frequency of more than $1 \%$ in public variation databases including the 1000 Genomes (Altshuler et al., 2015), the gnomAD database (Lek et al., 2016), the DGV (MacDonald, Ziman, Yuen, Feuk, \& Scherer, 2014) or our internal exome database, (ii) variants in $5^{\prime}$ and $3^{\prime}$ UTR, downstream, upstream, intronic and synonymous locations without pathogenic prediction of local splice effect. Structural variants were predicted using CANOES (Backenroth et al., 2014) and annotated by AnnotSV 2.0 (Geoffroy, Herenger, et al., 2018). Our analysis considered all inheritance modes but given that 2 families were consanguineous we focused on compound heterozygous and homozygous variants consistent with a recessive transmission. Variations have been classified according to the ACMG 
guidelines (Richards et al., 2015) and only class 3 (likely pathogenic) and class 4 (pathogenic) have been considered.

\section{Homozygosity mapping}

Based on the WES data, homozygosity mapping has been performed using PLINK 1.9 (Chang et al., 2015). We followed the parameters described by Kancheva et al (Kancheva et al., 2016). The results are available in Supp. Table 2.

\section{Cell culture}

Skin fibroblasts from patient A.IV-1 and age-matched healthy control fibroblasts cells were grown in DMEM supplemented with $10 \%$ fetal calf serum (FCS) and 1\% PSG (penicillin-streptomycinglutamin). To induce primary cilium formation, the cells were deprived of serum by growth for $24 \mathrm{~h}$ in DMEM with $1 \%$ PSG but only $0.1 \%$ FCS. In order to activate the Hh pathway, the cells were incubated in a DMEM medium with 1\% PSG without serum (-FCS) and with SAG (100nM). SAG (Smoothened Agonist) has been resuspended in DMSO at a stock concentration of $4 \mathrm{mM}$.

\section{RNA extraction, cDNA synthesis, q-PCR and Taqman}

For RNA seq validation, cells have been cultured in normal condition DMEM 1\% PSG, 10\% FCS. Total RNA was prepared from patient A.IV-1 fibroblast pellets (technical triplicate) and three controls pellets (biological triplicate) using QIAshredder Kit (Qiagen Ref 79654) and RNeasy Kit (Qiagen Ref 74104) followed by a DNAse treatment with the TURBO DNA-free ${ }^{T M}$. Experiments have been repeated 3 times. For the Hh genes expression analysis (qRT-PCR), cells have been cultured in DMEM 1\% PSG (-FCS) + SAG (100nM). Total RNA was prepared as described above and experiments have been repeated twice with 2 different control pellets (biological duplicate) 
and one patient pellet (technical triplicate). RNA integrity was assessed by gel electrophoresis and RNA concentration by Eppendorf Biophotometer Plus with the Hellma® Tray Cell. Reverse transcription of $1 \mu \mathrm{g}$ total RNA to cDNA was performed using the BioRad iScript ${ }^{\mathrm{TM}}$ CDNA synthesis kit. Real-time quantitative polymerase chain reaction amplification was performed in a BioRad CFX96 TM Real-Time System using the $\mathrm{iQ}^{\mathrm{TM}}$ SYBR® Green Supermix and primer sets (Supp. Table 3) optimized for tested targets for SYBR Green-based real-time PCR for the real-time PCR. The normalized fold expression of the target gene was calculated using the comparative cycle threshold $\left(\mathrm{C}_{\mathrm{t}}\right)$ method by normalizing target mRNA $\mathrm{C}_{\mathrm{t}}$ to those for both GAPDH and HPRT reference gene using the CFX Manager Software Version 1.5 and excel calculation. Statistical analysis have been done using the "Graphpad prism" software.

\section{Immunocytochemistry and fluorescence microscopy}

A.IV-1 and control ciliated fibroblasts were seeded on $12 \mathrm{~mm}$ diameter cover slips. Ciliogenesis has been induced by $24 \mathrm{~h}$ incubation in a DMEM, 1\% PSG medium, without serum (-FCS). Then, cells have been fixed with PFA-PBS 4\%. Subsequently, the cells were stained with the ciliary markers anti-acetylated alpha tubulin (Lys40) mouse monoclonal primary antibody (1:200, \# 322700 Invitrogen) and anti-EVC2 rabbit polyclonal primary antibody (1:250, \# ab198930, Abcam). As secondary antibody, donkey anti-mouse Alexa 594 (1:1000, Invitrogen) and donkey anti-rabbit DyLight 488 (1:1000, \#611-741-127, Rockland - Tebu-bio) were used. Ciliated cells count between A.IV-1 and control was performed manually using $~ 100$ cells per genotype (exact Fisher test was applied). Counts of EVC2 colocalization at the base of the cilia was performed manually using also $\sim 100$ cells per genotype in triplicate (Student t-test has been applied). Pictures were taken with a Zeiss axio imager Z2 and the analysis with the ZEN2012 software (Carl Zeiss Inc., Oberkochen, Germany). 


\section{Transcriptome analysis}

RNA samples were extracted from two fibroblast replicates of A.IV-1 (A.IV-1.1 and A.IV-1.2), and replicates from 6 individuals affected by other ciliopathies or intellectual disabilities of known molecular origin (ARN1.1, ARN1.2, ARN2.1, ARN2.2, ARN3.1, ARN3.2, ARN4.1, ARN4.2, ARN5.1, ARN6.1, ARN6.2) using TRI reagent $₫$ (Molecular Research Center) or using the RNeasy Mini Kit Qiagen®. Both protocols included an additional step of DNAse I recombinant treatment (Sigma-Aldrich $®$ ). The integrity of the RNA was visualized on a $1 \%$ bleach agarose gel by electrophoresis (Aranda et al., 2012). Quantification and further quality analysis were performed using the Nanodrop $®$. Samples should have a 260/280 ratios around 2 and a 260/230 ratio above 1.7. The integrity and quality of the RNA were also evaluated by running samples on a RNA 6000 Nano Chip on the Bioanalyzer (Agilent Technologies) and samples should have a RNA integrity number (RIN) equal or above 8. Library preparation was performed at the GenomEast platform at the Institute of Genetics and Molecular and Cellular Biology (Strasbourg, France), using the TruSeq ${ }^{\circledR}$ stranded mRNA sample preparation kit (Illumina) starting from $1 \mu \mathrm{g}$ of extracted total RNA. Libraries were then $100+75 b p$ paired-end sequenced, with 2 samples per lane on an Illumina Hiseq4000 sequencer. The corresponding bioinformatics pipeline is described in detail in the supplementary information.

\section{Zebrafish stocks}

Fish were bred and raised at $28.5^{\circ} \mathrm{C}$ as described previously (Westerfield, 1993). The AB wildtype line (European Zebrafish Resource Centre (EZRC), Karlsruhe) was used for all the experiments. Zebrafish husbandry and experimental procedures were performed in accordance with German animal protection regulations (Regierungspräsidium Karlsruhe, Germany, AZ35- 
9185.81/G-137/10). Fish were crossed pairwise. For experiments, fertilized eggs were raised in 1× Instant Ocean salt solution (Aquarium Systems, Inc.) supplemented with 200 M 1-phenyl 2thiourea (PTU) to suppress melanogenesis. For visualization of situs inversus, we used a transgenic line expressing GFP under the control of the skeletal and cardiac muscle specific promoter unc45b (Roostalu \& Strahle, 2012).

\section{In situ hybridization}

Whole-mount in situ hybridization was performed as previously described (Costa, Escaleira, Rodrigues, Manasfi, \& Mermelstein, 2002; Oxtoby \& Jowett, 1993). Ptc1 probe was obtained from (Concordet et al., 1996). wt1a probe was obtained from (Armant et al., 2013).

\section{Morpholinos and microinjections}

A search of the zebrafish genome (GRCz11) revealed a single orthologue of the human iqce gene (RefSeq: NM_001287204.1) encoding a protein sharing 58\% amino acid similarity with the human IQCE protein. Injections were performed as described previously (F. Muller et al., 1999). In brief, zebrafish eggs were collected shortly after being laid. Cleaned eggs were transferred to a petri dish with a minimal amount of water. Embryos were injected (FemtoJet; Eppendorf) through the chorion into the yolk at the one-cell stage. Injection needles were pulled from borosilicate glass capillary tubes with filament (Warner Instruments) using a micropipette puller (Sutter Instrument Co). Morpholinos were designed against the start codon of iqce (iqce-mo). Morpholino controls consist of the $5 \mathrm{bp}$ mismatch iqce-mo (iqce-mocont). Morpholinos (Gene Tools, LLC) were injected at the following concentrations: iqce-Mo: CAAGTTCTCCAGCTACCACAGACAT (0.6 $\mu \mathrm{M})$; iqcemocont; CAAcTTgTCgAGCTACCAgA cACAT $(0.6 \mu \mathrm{M})$. All dilutions were made in distilled water. Phenol red was added to the samples before injection ( $0.1 \%$ final concentration). Iqce-gfp plasmid was injected at the final concentration of $40 \mathrm{ng} / \mu \mathrm{l}$. When injected at $0.6 \mu \mathrm{M}$, neither the anti iqce 
nor the iqce-mocont (control) morpholino elicited necrosis, an unspecific effect of morpholino injections occasionally observed with some morpholinos (Robu et al., 2007). The efficiency of iqce-mo to block translation was assessed by co-injection of iqce-mo with a plasmid containing the iqce cDNA in-frame with gfp (iqce-gfp). As expected the embryos were depleted of GFP expression, indicating that the morpholinos indeed target efficiently iqce. In contrast, the 5mismatch iqce-mocont was unable to abrogate the translation of the fusion gene (Supp. Figure $3 A)$.

\section{PCR amplification}

Total RNA was isolated from 24 to $72 \mathrm{hpf}$ (hours post fertilization) embryos using Tri-reagent (Invitrogen, Carlsbad, CA). RT-PCR was carried out following standard protocols. For iqce in situ hybridization probe, a fragment of 580 bp was amplified by PCR with the following primers iqcewm5: GCTGCATCATGTAGGACGATG iqcewm3: CACTCTGAAGCTGACTCAAG, and cloned into pgem-t-easy vector (Promega). lqce full-length sequence was amplified from zebrafish cDNA with the following primers: iqce5: tcgGAATTCATGTCTGTGGTAGCTGGAGAAC; iqce3': agactcgagggTAAATCAAGTCA TCGGCACTG and cloned into pcs2+GFP with EcoRI-Xhol. For the rescue experiment iqce5'modif: tcgGAATTCATGTCGGTTGTAGCGGGCGAACTAG was used in combination with iqce3', and cloned into $p C S 2+G F P$ Morpholino-resistant iqce mRNA was synthesized with the SP6kit (Promega).

\section{Immunohistochemistry}

Fish embryos were fixed in 4\% paraformaldehyde/1x PBS one hour at room temperature. Embryos were permeabilized 7 min at $-20^{\circ} \mathrm{C}$ with cold acetone, rinsed 5 times, 5 min with $1 x$ PBS and blocked $4 \mathrm{~h}$ with BDP (1\% DMSO, 5\% BSA, 1x PBS). Incubation with primary antibody occurred 
over night at $4^{\circ} \mathrm{C}$, followed by thorough washing with PTW (1x PBS, $0.1 \%$ Tween 20$)$. Embryos were incubated with secondary antibody two hours at room temperature, and washed with PTW. Antibodies dilutions: Acetylated-tubulin (Sigma-Aldrich) 1/500; engrailed (Developmental Studies Hybridoma Bank, The University of lowa): 1/50; goat anti-rabbit alexa 488 (Invitrogen): 1/1000, goat anti-mouse alexa 488 (Invitrogen): 1/1000. For nucleus staining, we used Draq5 at 1/1000 dilution. Staining was analyzed by confocal microscopy (TCS SP2, Leica). For sectioning of the eyes, embryos were fixed in 4\% paraformaldehyde/PBS, progressively dehydrated in ethanol and stepwise transferred into Epon resin. Five $\mu \mathrm{m}$ sections were cut with a Leica microtome and stained with toluidine blue.

\section{RESULTS}

\section{Clinical features}

Three families with ciliopathy like phenotypes were referred to our laboratory as they remained unsolved following standard genetic testing. They all shared postaxial polydactyly (PAP) as a common feature but each presented other symptoms as described below (Figure 1A and Table 1). The affected patient of family A (A.IV-1) was born after an unremarkable pregnancy and is the only child of related Algerian parents. Bilateral polydactyly and brachydactyly of hands and feet were noted at birth associated with a syndactyly of the $2^{\text {nd }}$ and $3^{\text {rd }}$ digits of the feet. At the age of six years old, he was diagnosed with a decreased visual acuity and his ophthalmologic evaluation revealed retinal degeneration. He had hypogonadism and overweight was noted during his last clinical evaluation (15 years old). Family B is composed of three children presenting with PAP of hands and feet (bilateral for B.II-1 and unilateral for B.II-2 and B.II-3). The older one and the twin sister presented overweight respectively at the age of 7 and 5 years old in a context of obese parents. The ophthalmologic evaluation, including electroretinography, was normal for all the 
siblings. In family C, two affected children were reported. Individual C.IV-3 is the last child of related Moroccan parents and presented with PAP of both hands and one foot, learning disabilities and overweight. Her two sisters and parents share also the two last features. Her cousin, patient C.IV-4, born also from related parents is more severe. He had also PAP of the four limbs associated with renal anomalies, deafness and speech delay. The observed kidney disease consisted of a tubular nephropathy with nephrocalcinosis diagnosed at one year old evocative of a distal renal tubular acidosis. Deafness was detected at the age of two years old. His development was in the normal range, except for a language delay, in the context of hearing loss, which improved with speech therapy (Table 1).

The association of PAP and the other clinical features of each family member was assumed to be compatible with a syndromic ciliopathy (Wolff et al., 2004) and in particular to the Bardet-Biedl syndrome (BBS; MIM\# 209900). In line with the described phenotypes, the three families have been screened without success for the known BBS genes at that time (BBS1-BBS18) using either Sanger or targeted exome sequencing (J. Muller et al., 2010; Redin et al., 2012).

\section{Identification of IQCE pathogenic variations}

Whole exome sequencing (WES) was applied to all three families, generating on average a total of 81000 single nucleotide variants (SNVs), 11400 insertions-deletions (indels) and 21 structural variants (SV) (Supp. Table 1). In family A, as the proband (A.IV-1) was supposedly born from consanguineous parents, we identified 39 sizeable homozygous regions encompassing $238 \mathrm{Mb}$ (Supp. Table 2); supporting the assumption of consanguinity. In this case, autosomal recessive inheritance is more likely and we thus focused on homozygous variants, reducing the number of variants to 7 in 6 genes (DNAAF1, IQCE, MADD, SHANK2, TULP1 and ZDHHC2) (Supp. Table 1). A.IV-1 is affected by polydactyly, retinal dystrophy and hypogonadism, which made us, 
concentrate on 2 genes. The first one is IQCE (NM_152558.5), a gene that was just described as associated to PAP (Umair et al., 2017), with a homozygous 10 bp deletion in exon 12 c.895_904del (p.Val301Serfs ${ }^{\star}$ ) (Figure 1B) located in a conspicuous homozygous region of $2.4 \mathrm{Mb}$. This variant is present in gnomAD at a very low combined allele frequency $(0.0009534)$ with two homozygous carriers, which is compatible with the frequency of the disease. Subsequently, we analyzed IQCE exons 12 in 96 matched controls of Algerian ethnicity and could not find this variant (supplementary methods). Interestingly, the second gene is TULP1 (NM_001289395.1) a gene reported in Leber congenital amaurosis or retinitis pigmentosa (Hagstrom, North, Nishina, Berson, \& Dryja, 1998; Hanein et al., 2004) for which we identified a known homozygous pathogenic (class 5) variation, c.1198C>T (p.Arg400Trp), also located in a homozygous region of $8.5 \mathrm{Mb}$. This variant does not appear in public databases (gnomAD, 1000Genomes). The variants in both genes (IQCE and TULP1) segregated with the phenotypes within the family (Figure 1B) and in combination explained most of the clinical features of this individual. In the non-consanguineous family $\mathrm{B}$ (Figure 1A), WES revealed only 2 class-4 variations in IQCE at compound heterozygous state: c.895_904del (p.Val301Serfs*8) in exon 12 and c.1350_1353delAGAG, (p.Glu451Argfs*15) in exon 16 (Figure 1B). Both variations segregated in all affected members (B.II-1, B.II-2 and B.II3). The second variation (c.1350_1353delAGAG) was found in gnomAD with a frequency of 0.0002381 with 66 heterozygous carriers. In family C, WES analysis of the most severe case, C.IV-4 (Figure 1A), revealed a known homozygous variant in ATP6V1B1 (NM_001692.3), a gene that is associated with distal renal tubular acidosis with deafness (Vargas-Poussou et al., 2006) (dRTA; MIM\# 267300). This class-4 variant, c.175-1G>C, is located in a canonical splice site and is predicted to cause the skipping of exon 3, this variation was not found in public databases (gnomAD, 1000Genomes). Interestingly, the c.895_904del (p.Val301Serfs8) variation in IQCE that has been already observed in family A and B (respectively at the homozygous and compound heterozygous state) was only found in C.IV-4 at the heterozygous state (whereas no second pathogenic allele could be identified by cDNA sequencing and quantification, data not shown). 
The WES did not reveal any other variations in known PAP genes for this individual. Given that patient C.IV-3 had only PAP and given the C.IV-4 variation in IQCE, we sequenced the IQCE gene in C.IV-3 and surprisingly found the c.895_904del (p.Val301Serfs*8) at the homozygous state (Figure 1).

Given the recurrence of the c.895_904del (p.Val301Serfs $\left.{ }^{*} 8\right)$ variant in our cohort, we investigated a possible founder effect. Nevertheless, no common haplotype could be observed in the corresponding region in family $A$ and $B$ (Supp. Table 4). Analysis of the gnomAD record of this variation revealed its presence in different ethnical groups at a very low allele frequency: European (221/126532 alleles), Ashkenazi (12/10134 alleles), Latino (17/34392 alleles), African (6/23966 alleles), Finish (2/25788 alleles), south Asian (1/30782 alleles). The diverse origin of individuals with this IQCE variant and the differences in the haplotype suggested a recurrent mutational mechanism.

In order to better understand the effect of IQCE variants, we investigated the expression and subcellular localization of IQCE interacting partners in patient cells. IQCE is ubiquitously expressed in human adult tissues with higher levels of mRNA expression in lung, brain and hippocampus (Supp. Figure 1A). Endogenous IQCE is located at the base of the cilia and forms a complex with EFCAB7, to anchor the EVC-EVC2 sub-complex at the base of the cilia (EvC zone) for propagation of $\mathrm{Hh}$ signaling (Pusapati et al., 2014). We investigated the impact of IQCE deficiency in the formation of the primary cilia and localization of EVC-EVC2 sub-complex. Immunohistochemical studies on ciliated fibroblasts from individual A.IV-1 showed no differences in the number of ciliated cells (Supp. Figure 1B). Nevertheless, for $77 \%$ of patient's cells, we did not observe colocalization of EVC2 at the base of the cilia (Figure 2A and B), that presumably results in mislocalization of the EVC-EVC2 sub-complex and may compromise the Hh signaling.

\section{Transcriptome analysis and Hh signaling effect}


In order to get insight into the mechanistic impact of IQCE, we explored the transcriptome variation of individual A.IV-1 by RNA sequencing. We considered the possible impact of TULP1 variation. However, under physiological conditions, TULP1 is expressed exclusively in the retina, where it is involved in protein trafficking which is essential for the transport of rhodopsin (North, Naggert, Yan, Noben-Trauth, \& Nishina, 1997). This was confirmed as no TULP1 expression could be observed in the patient's fibroblasts. Given the IQCE genotype (frameshift) for A.IV-1 no IQCE expression could be detected. RNA differential expression analysis resulted in 98 significantly differentially expressed genes (Figure 2C and Supp. Table 5). None of the known IQCE interactors - EVC, EVC2, EFCAB7 and TCC23 (Breslow et al., 2018; Pusapati et al., 2018) - showed significant variations in their expression levels neither did the downstream effectors of the $\mathrm{Hh}$ signaling pathway GL/1, GL/2 and GL/3. We identified among the most significantly up and downregulated genes SLC35D3, SLC30A8 and GSTT1 respectively. SLC35D3 has been shown to be implicated in metabolic syndrome and obesity (Z. Zhang et al., 2014) and SLC30A8 is associated with a protective role against diabetes type2 (Flannick et al., 2014).

Regarding GSTT1, the protein catalyzes the conjugation of glutathione to a wide range of potential toxins as the first step in detoxification and is identified as risk factor for open angle glaucoma or ventricular diastolic dysfunction (Singh, Kumar, Tewari, \& Agarwal, 2018; Stamenkovic et al., 2018). In order to obtain functional clues about the IQCE protein modules, we applied functional enrichment analysis (WebGestalt 2019) (Wang, Vasaikar, Shi, Greer, \& Zhang, 2017); the overrepresentation analysis identified 10 significant clusters involving tissue development, ossification and skeletal system development among others (Figure 2D). A number of genes associated with Hh signaling and limb formation are included in these protein clusters (ALPL, ALDH1A1, FGF9, HOXC10, IGF2, IGFBP1, MECOM, MLLT3, NDP, SFRP1 and SMOC1) (Supp. Table 6). Selected genes with significant | log2 fold-change | > 0.5 were tested compared to controls by RT-qPCR and we confirmed the expression change of ALPL, GSTT1, IGF2, IQCE, MECOM, RSPO1 and SLC30A8 but we did not detect significant differences in FGF9 and SFRP1 (Figure 2E). 
In order to assess whether the Hh signaling is affected, we determined the expression levels of key genes in the patient's cells both under ciliary conditions and Hh activation (-FCS + SAG treatment). qRT-PCR revealed a significant under-expression of genes involved in the EVC-zone complex including EFCAB7, EVC, EVC2 and also GL/2 and GLI3 (Supp. Figure 2).

\section{Lack of iqce causes ciliary defects in zebrafish embryos}

Considering the ciliary localization of the human IQCE and encouraged by the previous studies, where genetic screens in zebrafish identified numerous defects of subpopulations of cilia (Zhao \& Malicki, 2007), we investigated expression and function of iqce in zebrafish (Danio rerio). We first assessed the iqce expression in the developing embryo. In situ hybridisation of iqce mRNA showed ubiquitous expression at the 10-somites and $20 \mathrm{hpf}$ stages with higher expression in the pronephric ducts at $20 \mathrm{hpf}$. At $48 \mathrm{hpf}$, higher levels of iqce mRNA were found in the pronephric ducts and in the mid- and hindbrain (Supp. Figure 3B).

A morpholino directed against the iqce start codon (iqce-mo) and a morpholino with a 5 mismatch control sequence (iqce-mocont) were used to elucidate the function of iqce in zebrafish embryos. Embryos injected with iqce-mo $(77 \%, \mathrm{n}=126)$ but not with the iqce-mocont control $(2 \%, \mathrm{n}=221)$ showed ventral curvature of the body axis (Figure 3A-B) which is often found in cilia mutants (Supp. Figure 3C). Occasionally we observed hydrocephalus (Figure 3B). In order to prove that the observed phenotype is due to the specific knock-down of iqce, we co-injected iqce-mo together with iqce mRNA resistant to iqce-mo. $54 \%$ of embryos exhibited a characteristic curved phenotype compared to $77 \%$ for iqce-mo alone (Figure 3D, $n=193$ ). We conclude from this experiment that the iqce-mo effectively abrogate the iqce expression. 
Bilaterally dilated kidney cyst were present in 37\% of iqce-mo morphants, but neither in uninjected nor in iqce-mocont injected embryos (Figure 3C and Supp. Figure 3D). These cysts are localized within the glomerulus as indicated by wt1a staining (Supp. Figure 3E).

We next examined the cilia of the pronephric duct. Embryos at $48 \mathrm{hpf}$ were stained with acetylatedtubulin antibody. In iqce-mocont and uninjected embryos, cilia are aligned along the duct forming a thin line (Figure 3E, G). In contrast, the cilia organization in iqce morphants was severely perturbed showing cilia oriented in all directions (Figure 3F). The misorientation and shortening of pronephric duct cilia can be due to dilation of the tubules and suggest that pronephric fluid flow may be affected (Zhao \& Malicki, 2007). We next tested whether other cilia are also affected, the dense ring of cilia on the epithelium surrounding the olfactory placode of 48-hpf wild-type larvae was strongly reduced in morphants (Figure $3 \mathrm{H}-\mathrm{J}$ ). In addition, the photoreceptor outer segment connects to the cell body via a narrow constriction that forms around a ciliary axoneme, and is known as the connecting cilium. Defects of photoreceptor cilia are associated with outer segment abnormalities and degeneration (Zhao \& Malicki, 2007). Therefore, we analyzed eye sections of 4 day old morphants. In non-injected embryos or control morphants all the layers are properly formed (Figure 3K). In contrast in iqce morphants, the lamination is disrupted and the retinal layer altered with no visible photoreceptor outer segment (Figure 3L).

The perturbation of motile cilia often results in a problem of left-right asymmetry. We thus examined the percentage of situs inversus in iqce morphants. iqce-mo or iqce-mocont were injected into embryos carrying a transgene that expresses GFP in the heart (-1.8 unc45b-TFP, (Roostalu \& Strahle, 2012). This allowed us to observe the looping of the heart. $44 \%$ of morphant embryos exhibited situs inversus (with the ventricle on the right and atrium on the left) or heterotaxia demonstrating that cilia from the Kupffer's vesicle, the fish equivalent of the mouse node, were affected by the iqce knock-down. In contrast only $3 \%$ and $6 \%$ of uninjected and iqcemocont injected embryos, respectively, showed reverse heart jogging (Figure 3M). 
In mutants with defective cilia (iguana) or ciliary motility (Irrc50), otoliths are frequently found ectopically, untethered or fused (Stooke-Vaughan, Huang, Hammond, Schier, \& Whitfield, 2012). We thus examined the number of otoliths in iqce morphants at $2 \mathrm{dpf}$. At $48 \mathrm{dpf}$ each ear should contains 2 otoliths and it is the case for $86 \%$ of the morphant. However, for $14 \%$ of them one of the two ears presented either one extra otolith ( 3 instead of 2 ) or only one ( 1 instead of 2 ) (data not shown, $\mathrm{n}=262$ embryos).

Cilia are necessary for normal activation as well as repression of the hedgehog $(\mathrm{Hh})$ signaling pathway (Goetz \& Anderson, 2010; Huang \& Schier, 2009; Wolff et al., 2004). It was shown that in the absence of the ciliary protein BBS1 and under stimulation of Hh, the ciliary localization of Smoothened ( $\mathrm{Smo}$ ) was further enhanced compared to wild-type cells (Nozaki, Katoh, Kobayashi, \& Nakayama, 2018). We thus examined the expression of the Hh receptor Patch1 (Ptc1) and the downstream gene engrailed (eng) in the developing somites in iqce morphants. At 10 somites stages, transcription of ptc1 was expanded to the myotome, whereas it is exclusively restricted to the adaxial cells in control or uninjected embryos (Figure 3N-PO and Supp. Figure 3F). Similarly, the domain of eng expression was significantly expanded among the fast muscle fibres by $24 \mathrm{hpf}$, indicative of an increased number of Hh-dependent muscle fast fibers (Figure $3 Q-S$ ). These results indicate that the Hh pathway is perturbed by the loss of iqce function.

\section{DISCUSSION}

An IQCE homozygous variant affecting the splicing of the gene was recently identified by whole exome sequencing in a single Pakistani family presenting with postaxial polydactyly type A limited to lower limb (Umair et al., 2017). Here, we report 3 additional families with pathogenic variations in IQCE referred initially as ciliopathies with postaxial polydactyly involving the upper and lower limbs. Interestingly, the 3 families shared the same c.895_904del (p.Val301Serfs $\left.{ }^{*} 8\right)$ variation in 
IQCE exon 12 in a homozygous or in a heterozygous state with another truncating variant. These two IQCE variations have already been observed in the largest public databases. The c.1350_1353del (p.Glu451Argfs*15) was described in Exome Variant Server (EVS) (Tennessen et al., 2012) with an average allele frequency $>1.5 \%$ with 90 homozygous calls. Initially, this overestimated allele frequency prevented its identification as candidate pathogenic variation. The higher false positive rate for indel calls was a known issue solved while integrating in ExAC/gnomAD (0.024\% and no homozygous calls).

The index case A.IV-1 had a suspected ciliopathy-like phenotype, due to the presence of retinitis pigmentosa (RP), hypogonadism, polydactyly and speech delay. Although the proband was assumed as a patient with one syndrome, the occurrence of pathogenic variations in both IQCE and TULP1 can explain independently the polydactyly and the RP. In the family C, the phenotypes of the patients were different; C.IV-3 has only PAP and overweight compared to her cousin who has PAP, deafness and renal anomalies. Phenotypic variations in a single family is not unusual in ciliopathies even with a single gene involved (Abdelhamed et al., 2013; Gonzalez-Del Pozo et al., 2014; Katsanis et al., 2001; Ware, Aygun, \& Hildebrandt, 2011; W. Zhang et al., 2018). The variations in IQCE, can clearly explain the isolated PAP in C.IV-3. However, in the C.IV-4 individual, the association of deafness and nephropathy could finally be explained by the identification of variations in the ATP6V1B1 gene (dRTA; MIM\# 267300), known to be responsible for renal tubular acidosis associated with deafness. Nevertheless, the identification of only a single pathogenic allele in IQCE and the absence of any other variation in known polydactyly genes do not provide any explanation to date for the PAP for this patient. Although, gene dosage and RNA expression have been performed for this individual revealing still $50 \%$ of the IQCE expression level (data not shown), one cannot rule out missed variation(s) either in IQCE or in another gene. Whole genome sequencing might reveal intronic, intragenic or structural variations missed by the WES (Geoffroy, Stoetzel, et al., 2018). These two examples illustrate well how the incidental co- 
occurrence of 2 different conditions can mimic the clinical features of a known Mendelian disease (DeLuca, Weed, Haas, Halder, \& Stone, 2015; Karaca et al., 2018). This point out also the importance for searching for multiple hits in whole exome or whole genome sequencing data to explain some apparent syndromic phenotype such as ciliopathy-like phenotype. The occurrence of the other clinical manifestations, overweight or learning disability, in these families seemed to be due to other factors than the presence of neurosensory impairment (RP or deafness). However, we should take into consideration the appreciable variations of systemic features in individuals affected by pathogenic variants in different ciliopathy genes (Carmi, Elbedour, Stone, \& Sheffield, 1995; Estrada-Cuzcano et al., 2012; Waters \& Beales, 2011).

Vertebrate limb patterning is controlled by two signaling centers, the zone of polarizing activity (ZPA) and the distal posterior mesenchyme and the apical ectodermal ridge (AER) at the distal limb bud. The ZPA and AER produce signaling molecules -Hh and FGFs respectively- that control the formation of anteroposterior and proximodistal limb axes in a cooperative manner (Yamamoto et al., 2012). Additionally, Hh can induce a positive feedback Igf signaling during osteoblast differentiation (mostly lgf2), regulating in this way the long bone growth (Fisher, Meyer, Garber, \& Dealy, 2005; Shi, Chen, Karner, \& Long, 2015). Until recently, the combination of temporal and spatial exposure to long-range Hh signaling was accepted as the main molecular mechanism underlying digit patterning (Ahn \& Joyner, 2004; Harfe et al., 2004). However, current investigations in chicken limb buds show that mesenchymal cells in the posterior limb bud produce actin-based filopodia that accumulate particles of $\mathrm{Hh}$, allowing the $\mathrm{Hh}$ particles to act at a distance without extracellular diffusion (Sanders, Llagostera, \& Barna, 2013). This study suggested that proper activation of the Hh target genes is more critical for digit patterning than diffusible longrange $\mathrm{Hh}$ morphogen threshold. The polydactyly phenotype of other carriers of IQCE variations supports the involvement of IQCE in the formation of limbs, process that is related to Hh signaling. At the base of the cilia, the EvC complex (constituted by EVC-EVC2 and EFCAB7-IQCE) is not essential for Hh target gene induction. However, it can reduce the Hh-stimulated induction of GLI1 
or PTCH1 (Pusapati et al., 2014). Interestingly, gene ontology analysis of misregulated transcripts revealed enrichment for skeletal system development. Notably, IGF2 - a critical protein for bone formation (Fisher et al., 2005; Shi et al., 2015)- is misregulated in absence of IQCE. Defects in IGF2 in humans cause Silver-Russell syndrome, ectrodactyly, under masculinized genitalia, developmental delay, and placental hypoplasia (Begemann et al., 2015; Yamoto et al., 2017). Additionally, we observed the up-regulation of IGFBP1 and IGFBP3, these two proteins bind IGF2 and their excess may limit IGF2 function (Chao \& D'Amore, 2008).

Knockdown of iqce in zebrafish showed the full spectrum of phenotypes associated with defective cilia: body curvature, kidney cysts, left right asymmetry, misdirected cilia in pronephric duct (Shi, Su, Lipschutz, \& Lobo, 2017) as well as retinal defects resembling very much those observed in bbs9 morphants (Veleri et al., 2012). In addition, full iqce knockout mouse showed short tibias, thin retinas, metabolic and behavioural alterations as well as polydactyly. Hh expression during limb development is pivotal for defining the identity and number of digits. When its expression is activated at an ectopic site in the limb bud this define a pathogenic mechanism that leads to preaxial polydactyly (Lettice et al., 2012). The iqce zebrafish model exhibits modified Hh signalling. Indeed, we observed a broadening of the Hh ptc1 receptor outside of the adaxial cell, as well as extended Engrailed expression. It is well known that the primary cilium acts as a Hh signal transduction machine (Goetz, Ocbina, \& Anderson, 2009). Recently it was shown that in a bbs1 $\mathrm{KO}$ cell line, the entry of Smo into cilia upon Hh stimulation was increased compared to the wild type cell line (Nozaki et al., 2018). This in turn activated ectopic engrailed expression as observed in iqce zebrafish morphants. This result was unexpected since Pusapati et al. provided evidence by analysing osteoblasts and the NIH3T3 cell line that the EFCAB7-IQCE module anchors the EVC-EVC2 complex in a signalling microdomain at the base of the cilia. Moreover, they showed that EFCAB7 depletion triggered the mislocalization of EVC-EVC2 within cilia and impaired activation of the transcription factor GLI2. These data suggest an impairment of Hh signalling 
rather than the gain of $\mathrm{Hh}$ signalling activity in the iqce mutant background that we observed in the zebrafish. However, in zebrafish, no Evc or Evc2 orthologues have been identified but only a paralog absent in human (Pusapati et al., 2014). Transcriptome analysis of the patient's cells under normal condition did not provide evidences of GLI genes modified expression compared to controls. However, under Hh activation, the patient's cells show a reduced expression of most of the key Hh pathway genes (GL/2, GL/3, EFCAB7, EVC, EVC2) (Supp. Figure 2). At the protein level, we observed a significant amount of EVC2 mislocalization at the base of the cilia (Figure 2B) as well as a reduced GLI2 protein expression (data not shown).

Blindness is not present in patients with only iqce defect. However, it has been previously observed in other cases that $\mathrm{KO}$ of genes in zebrafish can give additional phenotypes not observed in patients. For example, the zebrafish cc2d2 adult mutants exhibit a scoliosis that is not observed in Joubert syndrome (JBTS; MIM\# 213300) (Bachmann-Gagescu et al., 2011). The defective retina of iqce morphants is very similar to the bbs 9 morphant model showing a lack of lamination (Veleri et al., 2012). This observation fits with the implication of iqce in ciliopathies.

In conclusion, our findings confirm the role of IQCE in non-syndromic postaxial polydactyly, and its negative impact on the Hh signaling pathway. The differences between iqce zebrafish and mice models compared to humans raise the question of additional genetic factors. This study illustrates how detailed and accurate clinical data are needed to interpret exome-scale genetic results and translate those results in correct counselling and therapeutic intervention. 


\section{AVAILABILITY OF DATA AND MATERIAL}

Data generated or analysed during this study are included in the published article and the corresponding supplementary data. The raw sequencing data generated in the course of this study are not publicly available due to the protocol and the corresponding consents used that did not include such information. All variants have been submitted to ClinVar using the following range of accessions numbers SCV000929968 to SCV000929972 (https://www.ncbi.nlm.nih.gov/clinvar/). Anonymised NGS data and genomic variant data files will be made available upon request from qualified investigators studying the molecular basis of genomic disorders. Datasets can be obtained via the corresponding author on reasonable request.

\section{ACKNOWLEDGMENTS}

We would like to thank the patient's family for their participation and the patient's associations, Formicoeur, Bardet-Biedl France and Unadev, for their constant and strong support. We also would like to acknowledge the members of the diagnostic laboratories (molecular and cytogenetic units, in particular Manuela Antin, Anne-Sophie Leuvrey and Elsa Nourisson) at the University Hospital in Strasbourg for technical assistance. We thank Nadine Borel and the zebrafish house team. We also thank our collaborators for screening their negative cohort (KM. Bujakowska, E. Pierce, P. Beales, F. Hildebrandt). We thank the GenomEast platform (Strasbourg, France) especially Serge Vicaire for library preparation, Céline Keime for supervising the transcriptome analysis as well as Bernard Jost and Christelle Thibault. The computing resources for this work were provided by the BICS and BISTRO bioinformatics platforms in Strasbourg.

\section{FUNDING INFORMATION}


Whole exome sequencing was funded by Retina France (100 exomes Program), by Hôpitaux Universitaires de Strasbourg (API 2013-2014 HUS $N^{\circ}$ 5885). RNASeq analysis performed in patients with IQCE pathogenic variant and other patients were funded by Fondation Maladie Rare and Fondation Jérome Lejeune. CE and US were supported by the HGF Biointerfaces Programme. AEC is supported by Fondation JED-Belgique. CD is supported by a FRM grant (ECO20170637509).

\section{CONFLICTS OF INTEREST}

The authors declare no conflict of interest. 


\section{REFERENCES}

Abbasi, A. A. (2011). Evolution of vertebrate appendicular structures: Insight from genetic and palaeontological data. Dev Dyn, 240(5), 1005-1016. doi:10.1002/dvdy.22572

Abdelhamed, Z. A., Wheway, G., Szymanska, K., Natarajan, S., Toomes, C., Inglehearn, C., \& Johnson, C. A. (2013). Variable expressivity of ciliopathy neurological phenotypes that encompass MeckelGruber syndrome and Joubert syndrome is caused by complex de-regulated ciliogenesis, Shh and Wnt signalling defects. Hum Mol Genet, 22(7), 1358-1372. doi:10.1093/hmg/dds546

Ahn, S., \& Joyner, A. L. (2004). Dynamic changes in the response of cells to positive hedgehog signaling during mouse limb patterning. Cell, 118(4), 505-516. doi:10.1016/j.cell.2004.07.023

Altshuler, D. M., Durbin, R. M., Abecasis, G. R., Bentley, D. R., Chakravarti, A., Clark, A. G., . . Consortium, G. P. (2015). A global reference for human genetic variation. Nature, 526(7571), 68-+. doi:10.1038/nature15393

Armant, O., Marz, M., Schmidt, R., Ferg, M., Diotel, N., Ertzer, R., . . Rastegar, S. (2013). Genome-wide, whole mount in situ analysis of transcriptional regulators in zebrafish embryos. Dev Biol, 380(2), 351-362. doi:10.1016/j.ydbio.2013.05.006

Bachmann-Gagescu, R., Phelps, I. G., Stearns, G., Link, B. A., Brockerhoff, S. E., Moens, C. B., \& Doherty, D. (2011). The ciliopathy gene cc2d2a controls zebrafish photoreceptor outer segment development through a role in Rab8-dependent vesicle trafficking. Hum Mol Genet, 20(20), 4041-4055. doi:10.1093/hmg/ddr332

Backenroth, D., Homsy, J., Murillo, L. R., Glessner, J., Lin, E., Brueckner, M., .. Shen, Y. F. (2014). CANOES: detecting rare copy number variants from whole exome sequencing data. Nucleic Acids Research, 42(12). doi:ARTN e97 10.1093/nar/gku345

Barakat, M. T., Humke, E. W., \& Scott, M. P. (2010). Learning from Jekyll to control Hyde: Hedgehog signaling in development and cancer. Trends Mol Med, 16(8), 337-348. doi:10.1016/j.molmed.2010.05.003

Begemann, M., Zirn, B., Santen, G., Wirthgen, E., Soellner, L., Buttel, H. M., . . Eggermann, T. (2015). Paternally Inherited IGF2 Mutation and Growth Restriction. N Engl J Med, 373(4), 349-356. doi:10.1056/NEJMoa1415227

Breslow, D. K., Hoogendoorn, S., Kopp, A. R., Morgens, D. W., Vu, B. K., Kennedy, M. C., ... Nachury, M. V. (2018). A CRISPR-based screen for Hedgehog signaling provides insights into ciliary function and ciliopathies. Nat Genet. doi:10.1038/s41588-018-0054-7

Carmi, R., Elbedour, K., Stone, E. M., \& Sheffield, V. C. (1995). Phenotypic differences among patients with Bardet-Biedl syndrome linked to three different chromosome loci. Am J Med Genet, 59(2), 199203. doi:10.1002/ajmg.1320590216

Castilla, E., Paz, J., Mutchinick, O., Munoz, E., Giorgiutti, E., \& Gelman, Z. (1973). Polydactyly: a genetic study in South America. Am J Hum Genet, 25(4), 405-412. Retrieved from http://www.ncbi.nlm.nih.gov/pubmed/4716659

Chang, C. C., Chow, C. C., Tellier, L. C., Vattikuti, S., Purcell, S. M., \& Lee, J. J. (2015). Second-generation PLINK: rising to the challenge of larger and richer datasets. Gigascience, 4, 7. doi:10.1186/s13742015-0047-8

Chao, W., \& D'Amore, P. A. (2008). IGF2: epigenetic regulation and role in development and disease. Cytokine Growth Factor Rev, 19(2), 111-120. doi:10.1016/j.cytogfr.2008.01.005

Concordet, J. P., Lewis, K. E., Moore, J. W., Goodrich, L. V., Johnson, R. L., Scott, M. P., \& Ingham, P. W. (1996). Spatial regulation of a zebrafish patched homologue reflects the roles of sonic hedgehog and protein kinase A in neural tube and somite patterning. Development, 122(9), 2835-2846. Retrieved from http://www.ncbi.nlm.nih.gov/pubmed/8787757 
Costa, M. L., Escaleira, R. C., Rodrigues, V. B., Manasfi, M., \& Mermelstein, C. S. (2002). Some distinctive features of zebrafish myogenesis based on unexpected distributions of the muscle cytoskeletal proteins actin, myosin, desmin, alpha-actinin, troponin and titin. Mechanisms of Development, 116(1-2), 95-104. doi:Pii S0925-4773(02)00149-1

Doi 10.1016/S0925-4773(02)00149-1

Deluca, A. P., Weed, M. C., Haas, C. M., Halder, J. A., \& Stone, E. M. (2015). Apparent Usher Syndrome Caused by the Combination of BBS1-Associated Retinitis Pigmentosa and SLC26A4-Associated Deafness. JAMA Ophthalmol, 133(8), 967-968. doi:10.1001/jamaophthalmol.2015.1463

DePristo, M. A., Banks, E., Poplin, R., Garimella, K. V., Maguire, J. R., Hartl, C., . . . Daly, M. J. (2011). A framework for variation discovery and genotyping using next-generation DNA sequencing data. Nat Genet, 43(5), 491-+. doi:10.1038/ng.806

Estrada-Cuzcano, A., Koenekoop, R. K., Senechal, A., De Baere, E. B., de Ravel, T., Banfi, S., ... Klevering, B. J. (2012). BBS1 mutations in a wide spectrum of phenotypes ranging from nonsyndromic retinitis pigmentosa to Bardet-Biedl syndrome. Arch Ophthalmol, 130(11), 1425-1432. doi:10.1001/archophthalmol.2012.2434

Etard, C., Roostalu, U., \& Strahle, U. (2010). Lack of Apobec2-related proteins causes a dystrophic muscle phenotype in zebrafish embryos. J Cell Biol, 189(3), 527-539. doi:10.1083/jcb.200912125

Fisher, M. C., Meyer, C., Garber, G., \& Dealy, C. N. (2005). Role of IGFBP2, IGF-I and IGF-II in regulating long bone growth. Bone, 37(6), 741-750. doi:10.1016/j.bone.2005.07.024

Flannick, J., Thorleifsson, G., Beer, N. L., Jacobs, S. B., Grarup, N., Burtt, N. P., . . Altshuler, D. (2014). Lossof-function mutations in SLC30A8 protect against type 2 diabetes. Nat Genet, 46(4), 357-363. doi:10.1038/ng.2915

Geoffroy, V., Herenger, Y., Kress, A., Stoetzel, C., Piton, A., Dollfus, H., \& Muller, J. (2018). AnnotSV: An integrated tool for Structural Variations annotation. Bioinformatics. doi:10.1093/bioinformatics/bty304

Geoffroy, V., Pizot, C., Redin, C., Piton, A., Vasli, N., Stoetzel, C., ... Muller, J. (2015). VaRank: a simple and powerful tool for ranking genetic variants. PeerJ, 3, e796. doi:10.7717/peerj.796

Geoffroy, V., Stoetzel, C., Scheidecker, S., Schaefer, E., Perrault, I., Bar, S., . . Muller, J. (2018). Wholegenome sequencing in patients with ciliopathies uncovers a novel recurrent tandem duplication in IFT140. Hum Mutat, 39(7), 983-992. doi:10.1002/humu.23539

Goetz, S. C., \& Anderson, K. V. (2010). The primary cilium: a signalling centre during vertebrate development. Nature Reviews Genetics, 11(5), 331-344. doi:10.1038/nrg2774

Goetz, S. C., Ocbina, P. J. R., \& Anderson, K. V. (2009). The Primary Cilium as a Hedgehog Signal Transduction Machine. Primary Cilia, 94, 199-+. doi:10.1016/S0091-679x(08)94010-3

Gonzalez-Del Pozo, M., Mendez-Vidal, C., Santoyo-Lopez, J., Vela-Boza, A., Bravo-Gil, N., Rueda, A., . . . Antinolo, G. (2014). Deciphering intrafamilial phenotypic variability by exome sequencing in a Bardet-Biedl family. Mol Genet Genomic Med, 2(2), 124-133. doi:10.1002/mgg3.50

Hagstrom, S. A., North, M. A., Nishina, P. L., Berson, E. L., \& Dryja, T. P. (1998). Recessive mutations in the gene encoding the tubby-like protein TULP1 in patients with retinitis pigmentosa. Nat Genet, 18(2), 174-176. doi:10.1038/ng0298-174

Hanein, S., Perrault, I., Gerber, S., Tanguy, G., Barbet, F., Ducroq, D., . . Kaplan, J. (2004). Leber congenital amaurosis: comprehensive survey of the genetic heterogeneity, refinement of the clinical definition, and genotype-phenotype correlations as a strategy for molecular diagnosis. Hum Mutat, 23(4), 306-317. doi:10.1002/humu.20010

Harfe, B. D., Scherz, P. J., Nissim, S., Tian, H., McMahon, A. P., \& Tabin, C. J. (2004). Evidence for an expansion-based temporal Shh gradient in specifying vertebrate digit identities. Cell, 118(4), 517528. doi:10.1016/j.cell.2004.07.024 
Huang, P., \& Schier, A. F. (2009). Dampened Hedgehog signaling but normal Wnt signaling in zebrafish without cilia. Development, 136(18), 3089-3098. doi:10.1242/dev.041343

Ingham, P. W., Nakano, Y., \& Seger, C. (2011). Mechanisms and functions of Hedgehog signalling across the metazoa. Nature Reviews Genetics, 12(6), 393-406. doi:10.1038/nrg2984

Kancheva, D., Atkinson, D., De Rijk, P., Zimon, M., Chamova, T., Mitev, V., . . Jordanova, A. (2016). Novel mutations in genes causing hereditary spastic paraplegia and Charcot-Marie-Tooth neuropathy identified by an optimized protocol for homozygosity mapping based on whole-exome sequencing. Genet Med, 18(6), 600-607. doi:10.1038/gim.2015.139

Karaca, E., Posey, J. E., Coban Akdemir, Z., Pehlivan, D., Harel, T., Jhangiani, S. N., . . Lupski, J. R. (2018). Phenotypic expansion illuminates multilocus pathogenic variation. Genet Med. doi:10.1038/gim.2018.33

Katsanis, N., Ansley, S. J., Badano, J. L., Eichers, E. R., Lewis, R. A., Hoskins, B. E., . . Lupski, J. R. (2001). Triallelic inheritance in Bardet-Biedl syndrome, a Mendelian recessive disorder. Science, 293(5538), 2256-2259. doi:10.1126/science.1063525

Lek, M., Karczewski, K. J., Minikel, E. V., Samocha, K. E., Banks, E., Fennell, T., . . Consortium, E. A. (2016). Analysis of protein-coding genetic variation in 60,706 humans. Nature, 536(7616), 285-+. doi:10.1038/nature19057

Lettice, L. A., Williamson, I., Wiltshire, J. H., Peluso, S., Devenney, P. S., Hill, A. E., . . Hill, R. E. (2012). Opposing functions of the ETS factor family define Shh spatial expression in limb buds and underlie polydactyly. Dev Cell, 22(2), 459-467. doi:10.1016/j.devcel.2011.12.010

MacDonald, J. R., Ziman, R., Yuen, R. K. C., Feuk, L., \& Scherer, S. W. (2014). The Database of Genomic Variants: a curated collection of structural variation in the human genome. Nucleic Acids Research, 42(D1), D986-D992. doi:10.1093/nar/gkt958

Malik, S. (2014). Polydactyly: phenotypes, genetics and classification. Clin Genet, 85(3), 203-212. doi:10.1111/cge.12276

Malik, S., Ullah, S., Afzal, M., Lal, K., \& Haque, S. (2014). Clinical and descriptive genetic study of polydactyly: a Pakistani experience of 313 cases. Clin Genet, 85(5), 482-486. doi:10.1111/cge.12217

Metsalu, T., \& Vilo, J. (2015). ClustVis: a web tool for visualizing clustering of multivariate data using Principal Component Analysis and heatmap. Nucleic Acids Research, 43(W1), W566-570. doi:10.1093/nar/gkv468

Muller, F., Blader, P., Rastegar, S., Fischer, N., Knochel, W., \& Strahle, U. (1999). Characterization of zebrafish smad1, smad2 and smad5: the amino-terminus of Smad1 and Smad5 is required for specific function in the embryo. Mechanisms of Development, 88(1), 73-88. doi:Doi 10.1016/S0925-4773(99)00173-2

Muller, J., Stoetzel, C., Vincent, M. C., Leitch, C. C., Laurier, V., Danse, J. M., . . . Dollfus, H. (2010). Identification of 28 novel mutations in the Bardet-Biedl syndrome genes: the burden of private mutations in an extensively heterogeneous disease. Hum Genet, 127(5), 583-593. doi:10.1007/s00439-010-0804-9

North, M. A., Naggert, J. K., Yan, Y., Noben-Trauth, K., \& Nishina, P. M. (1997). Molecular characterization of TUB, TULP1, and TULP2, members of the novel tubby gene family and their possible relation to ocular diseases. Proc Natl Acad Sci U S A, 94(7), 3128-3133. Retrieved from http://www.ncbi.nlm.nih.gov/pubmed/9096357

Nozaki, S., Katoh, Y., Kobayashi, T., \& Nakayama, K. (2018). BBS1 is involved in retrograde trafficking of ciliary GPCRs in the context of the BBSome complex. PLoS One, 13(3), e0195005. doi:10.1371/journal.pone.0195005 
Oxtoby, E., \& Jowett, T. (1993). Cloning of the Zebrafish Krox-20 Gene (Krx-20) and Its Expression during Hindbrain Development. Nucleic Acids Research, 21(5), 1087-1095. doi:DOI $10.1093 /$ nar/21.5.1087

Petrova, R., \& Joyner, A. L. (2014). Roles for Hedgehog signaling in adult organ homeostasis and repair. Development, 141(18), 3445-3457. doi:10.1242/dev.083691

Phadke, S. R., \& Sankar, V. H. (2010). Polydactyly and genes. Indian J Pediatr, 77(3), 277-281. doi:10.1007/s12098-010-0033-1

Pusapati, G. V., Hughes, C. E., Dorn, K. V., Zhang, D., Sugianto, P., Aravind, L., \& Rohatgi, R. (2014). EFCAB7 and IQCE regulate hedgehog signaling by tethering the EVC-EVC2 complex to the base of primary cilia. Dev Cell, 28(5), 483-496. doi:10.1016/j.devcel.2014.01.021

Pusapati, G. V., Kong, J. H., Patel, B. B., Krishnan, A., Sagner, A., Kinnebrew, M., . . Rohatgi, R. (2018). CRISPR Screens Uncover Genes that Regulate Target Cell Sensitivity to the Morphogen Sonic Hedgehog. Dev Cell, 44(2), 271. doi:10.1016/j.devcel.2018.01.002

Redin, C., Le Gras, S., Mhamdi, O., Geoffroy, V., Stoetzel, C., Vincent, M. C., . . Muller, J. (2012). Targeted high-throughput sequencing for diagnosis of genetically heterogeneous diseases: efficient mutation detection in Bardet-Biedl and Alstrom syndromes. J Med Genet, 49(8), 502-512. doi:10.1136/jmedgenet-2012-100875

Richards, S., Aziz, N., Bale, S., Bick, D., Das, S., Gastier-Foster, J., . . Committee, A. L. Q. A. (2015). Standards and guidelines for the interpretation of sequence variants: a joint consensus recommendation of the American College of Medical Genetics and Genomics and the Association for Molecular Pathology. Genet Med, 17(5), 405-424. doi:10.1038/gim.2015.30

Robu, M. E., Larson, J. D., Nasevicius, A., Beiraghi, S., Brenner, C., Farber, S. A., \& Ekker, S. C. (2007). p53 activation by knockdown technologies. PLoS Genet, 3(5), e78. doi:10.1371/journal.pgen.0030078

Roostalu, U., \& Strahle, U. (2012). In Vivo Imaging of Molecular Interactions at Damaged Sarcolemma. Dev Cell, 22(3), 515-529. doi:10.1016/j.devcel.2011.12.008

Sanders, T. A., Llagostera, E., \& Barna, M. (2013). Specialized filopodia direct long-range transport of SHH during vertebrate tissue patterning. Nature, 497(7451), 628-632. doi:10.1038/nature12157

Shaheen, R., Szymanska, K., Basu, B., Patel, N., Ewida, N., Faqeih, E., . . . Alkuraya, F. S. (2016). Characterizing the morbid genome of ciliopathies. Genome Biol, 17(1), 242. doi:10.1186/s13059016-1099-5

Shi, Y., Chen, J., Karner, C. M., \& Long, F. (2015). Hedgehog signaling activates a positive feedback mechanism involving insulin-like growth factors to induce osteoblast differentiation. Proc Natl Acad Sci U S A, 112(15), 4678-4683. doi:10.1073/pnas.1502301112

Shi, Y., Su, Y., Lipschutz, J. H., \& Lobo, G. P. (2017). Zebrafish as models to study ciliopathies of the eye and kidney. Clin Nephrol Res, 1(1), 6-9. Retrieved from http://www.ncbi.nlm.nih.gov/pubmed/29553143

Singh, M. M., Kumar, R., Tewari, S., \& Agarwal, S. (2018). Association of GSTT1/GSTM1 and ApoE variants with left ventricular diastolic dysfunction in thalassaemia major patients. Hematology, 1-6. doi:10.1080/10245332.2018.1502397

Stamenkovic, M., Lukic, V., Suvakov, S., Simic, T., Sencanic, I., Pljesa-Ercegovac, M., . . Djukic, T. (2018). GSTM1-null and GSTT1-active genotypes as risk determinants of primary open angle glaucoma among smokers. Int J Ophthalmol, 11(9), 1514-1520. doi:10.18240/ijo.2018.09.14

Stooke-Vaughan, G. A., Huang, P., Hammond, K. L., Schier, A. F., \& Whitfield, T. T. (2012). The role of hair cells, cilia and ciliary motility in otolith formation in the zebrafish otic vesicle. Development, 139(10), 1777-1787. doi:10.1242/dev.079947

Tennessen, J. A., Bigham, A. W., O'Connor, T. D., Fu, W., Kenny, E. E., Gravel, S., . . Project, N. E. S. (2012). Evolution and functional impact of rare coding variation from deep sequencing of human exomes. Science, 337(6090), 64-69. doi:10.1126/science.1219240 
Ullah, I., Kakar, N., Schrauwen, I., Hussain, S., Chakchouk, I., Liaqat, K., . . Leal, S. M. (2019). Variants in KIAA0825 underlie autosomal recessive postaxial polydactyly. Hum Genet. doi:10.1007/s00439019-02000-0

Umair, M., Ahmad, F., Bilal, M., Ahmad, W., \& Alfadhel, M. (2018). Clinical Genetics of Polydactyly: An Updated Review. Front Genet, 9, 447. doi:10.3389/fgene.2018.00447

Umair, M., Shah, K., Alhaddad, B., Haack, T. B., Graf, E., Strom, T. M., . . . Ahmad, W. (2017). Exome sequencing revealed a splice site variant in the IQCE gene underlying post-axial polydactyly type $A$ restricted to lower limb. Eur J Hum Genet, 25(8), 960-965. doi:10.1038/ejhg.2017.83

Vargas-Poussou, R., Houillier, P., Le Pottier, N., Strompf, L., Loirat, C., Baudouin, V., .. Blanchard, A. (2006). Genetic investigation of autosomal recessive distal renal tubular acidosis: evidence for early sensorineural hearing loss associated with mutations in the ATP6VOA4 gene. J Am Soc Nephrol, 17(5), 1437-1443. doi:10.1681/ASN.2005121305

Veleri, S., Bishop, K., Nogare, D. E. D., English, M. A., Foskett, T. J., Chitnis, A., . . Swaroop, A. (2012). Knockdown of Bardet-Biedl Syndrome Gene BBS9/PTHB1 Leads to Cilia Defects. PLoS One, 7(3). doi:ARTN e34389 10.1371/journal.pone.0034389

Wang, J., Vasaikar, S., Shi, Z., Greer, M., \& Zhang, B. (2017). WebGestalt 2017: a more comprehensive, powerful, flexible and interactive gene set enrichment analysis toolkit. Nucleic Acids Research, 45(W1), W130-W137. doi:10.1093/nar/gkx356

Ware, S. M., Aygun, M. G., \& Hildebrandt, F. (2011). Spectrum of clinical diseases caused by disorders of primary cilia. Proc Am Thorac Soc, 8(5), 444-450. doi:10.1513/pats.201103-025SD

Waters, A. M., \& Beales, P. L. (2011). Ciliopathies: an expanding disease spectrum. Pediatr Nephrol, 26(7), 1039-1056. doi:10.1007/s00467-010-1731-7

Westerfield, M. (1993). The zebrafish book: a guide for the laboratory use of zebrafish (Brachydanio reriro). 300.

Wolff, C., Roy, S., Lewis, K. E., Schauerte, H., Joerg-Rauch, G., Kirn, A., . . Ingham, P. W. (2004). iguana encodes a novel zinc-finger protein with coiled-coil domains essential for Hedgehog signal transduction in the zebrafish embryo. Genes \& Development, 18(13), 1565-1576. doi:10.1101/gad.296004

Yamamoto, M., Matsuzaki, T., Takahashi, R., Adachi, E., Maeda, Y., Yamaguchi, S., . . Noda, M. (2012). The transformation suppressor gene Reck is required for postaxial patterning in mouse forelimbs. Biol Open, 1(5), 458-466. doi:10.1242/bio.2012638

Yamoto, K., Saitsu, H., Nakagawa, N., Nakajima, H., Hasegawa, T., Fujisawa, Y., . . Ogata, T. (2017). De novo IGF2 mutation on the paternal allele in a patient with Silver-Russell syndrome and ectrodactyly. Hum Mutat, 38(8), 953-958. doi:10.1002/humu.23253

Zhang, W., Taylor, S. P., Ennis, H. A., Forlenza, K. N., Duran, I., Li, B., . . Cohn, D. H. (2018). Expanding the genetic architecture and phenotypic spectrum in the skeletal ciliopathies. Hum Mutat, 39(1), 152166. doi:10.1002/humu.23362

Zhang, Z., Hao, C. J., Li, C. G., Zang, D. J., Zhao, J., Li, X. N., . . Li, W. (2014). Mutation of SLC35D3 causes metabolic syndrome by impairing dopamine signaling in striatal D1 neurons. PLoS Genet, 10(2), e1004124. doi:10.1371/journal.pgen.1004124

Zhao, C. T., \& Malicki, J. (2007). Genetic defects of pronephric cilia in zebrafish. Mechanisms of Development, 124(7-8), 605-616. doi:10.1016/j.mod.2007.04.004 


\begin{tabular}{|c|c|c|c|c|c|c|}
\hline & \multirow{2}{*}{$\begin{array}{c}\text { Family A } \\
\text { A.IV-1 }\end{array}$} & \multicolumn{3}{|c|}{ Family B } & \multicolumn{2}{|c|}{ Family C } \\
\hline & & B.II-1 & B.II-2 & B.II-3 & C.IV-3 & C.IV-4 \\
\hline IQCE & $\begin{array}{l}\text { p.[Val301Serfs*8]; } \\
\text { [Val301Serfs*8] }\end{array}$ & $\begin{array}{c}\text { p.[Val301Serfs*8]; } \\
\text { [Glu451fs*15] }\end{array}$ & $\begin{array}{c}\text { p.[Val301Serfs*8]; } \\
\text { [Glu451fs*15] }\end{array}$ & $\begin{array}{c}\text { p.[Val301Serfs*8]; } \\
\text { [Glu451fs*15] }\end{array}$ & $\begin{array}{l}\text { p.[Val301Serfs*8]; } \\
\text { [Val301Serfs*8] }\end{array}$ & p.[Val301Serfs*8];[=] \\
\hline Other gene & $\begin{array}{c}\text { TULP1 } \\
\text { p.[Arg400Trp];[Arg400Trp] }\end{array}$ & & & & & $\begin{array}{c}\text { ATP6V1B1 } \\
\text { c. }[175-1 \mathrm{G}>\mathrm{C}] ;[175-1 \mathrm{G}>\mathrm{C}]\end{array}$ \\
\hline \multicolumn{7}{|c|}{ Clinical features } \\
\hline \multicolumn{7}{|c|}{ Hands and feet abnormalities } \\
\hline Postaxial polydactyly & $\mathrm{Y}$ & $\mathrm{Y}$ & $\mathrm{Y}$ & $\mathrm{Y}$ & $\mathrm{Y}$ & $\mathrm{Y}$ \\
\hline Hands & Y & Y & $Y^{*}$ & $\mathrm{Y}^{*}$ & Y & Y \\
\hline Feet & $\mathrm{Y}$ & $\mathrm{Y}$ & $\mathrm{Y}^{*}$ & $\mathrm{Y}^{*}$ & $\mathrm{Y}$ & $\mathrm{Y}$ \\
\hline Brachydactyly & Y & Y & $\mathrm{Y}$ & $\mathrm{Y}$ & $\mathrm{N}$ & $\mathrm{N}$ \\
\hline Syndactyly & $\mathrm{Y}$ & $\mathrm{N}$ & $\mathrm{N}$ & $\mathrm{Y}$ & $\mathrm{N}$ & $\mathrm{N}$ \\
\hline \multicolumn{7}{|l|}{ Neurosensory disease } \\
\hline Retinal dystrophy & $\mathrm{Y}$ & $\mathrm{N}$ & $\mathrm{N}$ & $\mathrm{N}$ & $\mathrm{N}$ & $\mathrm{N}$ \\
\hline Deafness & $\mathrm{N}$ & $\mathrm{N}$ & $\mathrm{N}$ & $\mathrm{N}$ & $\mathrm{N}$ & $\mathrm{Y}$ \\
\hline \multicolumn{7}{|l|}{ Pondostatural evaluation } \\
\hline Overweight & $\mathrm{Y}$ & $\mathrm{Y}$ & $\mathrm{Y}$ & $\mathrm{N}$ & $\mathrm{Y}$ & $\mathrm{N}$ \\
\hline \multicolumn{7}{|c|}{ Development psychomotor } \\
\hline Intellectual abnormalities & $\mathrm{N}$ & $\mathrm{N}$ & $\mathrm{N}$ & $\mathrm{N}$ & $\mathrm{N}$ & $\mathrm{N}$ \\
\hline Learning disabilities & $\mathrm{N}$ & $\mathrm{N}$ & $\mathrm{N}$ & $\mathrm{N}$ & $\mathrm{Y}$ & $\mathrm{N}$ \\
\hline Speech delay & $\mathrm{Y}$ & $\mathrm{N}$ & $\mathrm{N}$ & $\mathrm{N}$ & $\mathrm{Y}$ & $\mathrm{Y}$ \\
\hline Ataxia & $\mathrm{N}$ & $\mathrm{N}$ & $\mathrm{N}$ & $\mathrm{N}$ & $\mathrm{N}$ & $\mathrm{N}$ \\
\hline \multicolumn{7}{|l|}{ Kidney function } \\
\hline Renal abnormality & $\mathrm{N}$ & $\mathrm{N}$ & $\mathrm{N}$ & $\mathrm{N}$ & $\mathrm{N}$ & $\begin{array}{c}\text { Tubulopathy - } \\
\text { Nephrocalcinosis }\end{array}$ \\
\hline \multicolumn{7}{|l|}{ Genital abnormalities } \\
\hline Hypogonadism & Micropenis & $\mathrm{N}$ & & & $\mathrm{N}$ & $\mathrm{N}$ \\
\hline
\end{tabular}

Table 1. Clinical characteristic of individuals with variants in IQCE. $Y=y e s, N=$ not present, " $=$ unilateral 
Figure 1. Clinical description and molecular genetic characterization of IQCE variations. (A) Graphic representation of three autosomal recessive families with complicated polydactyly in whom IQCE, TULP1 and ATP6V1B1 variations were identified. Sanger sequencing traces confirming the presence of the pathogenic variant and segregation in available family members is demonstrated. The "WT" symbol represents wild type and "M" symbol represents pathogenic variant. Blackened symbols indicate affected individuals. (B) Genomic structure (NC_000007.14) and schematic representation of the protein layout (NP_689771.3) of IQCE and location of currently known disease-associated variant (in bold, novel pathogenic variants identified in this study). Topology of IQCE represents one hydrophobic segment (HS), coil-coil conserved domain regions, three IQ calmodulin binding motifs (IQ) and one acid region (AR).

Figure 2. (A). Immunostaining of A.IV-1 and control ciliated fibroblasts, antibodies against human EVC2 (in green) and the ciliary marker anti acetylated alpha tubulin (in red), revealed that in absence of endogenous IQCE, EVC2 fails to localize at the base of the cilia. (B). Count of mislocalized EVC2 at the base of the cilia in fibroblasts under ciliated conditions (-FCS) between A.IV-1 and control (3 times $\sim 100$ cells per condition). Statistical significance was determined using the Student's t-test, NS, non-significant, $\left.p=0.0004{ }^{(* * *}\right)$. (C). Heat map generated using ClustVis (Metsalu \& Vilo, 2015) from the transcriptome sequencing data reflecting gene counts of the significantly differentially expressed genes across patient and control samples. (D). Volcano plot of overrepresentation enrichment analysis of significantly misregulated transcripts in A.IV-1 fibroblast compared to wild type. These results were obtained using WebGestalt 2019, (Supp. Table 6). (E). Real-time quantitative polymerase chain reaction analysis from 3 technical replicates of A.IV-1 and 3 controls fibroblasts showed relative expression levels (normalized to GAPDH) of 9 significantly differentially expressed genes, data includes $\pm S D$. NS $=$ no significative, ${ }^{*}=p$-value $<0.05,{ }^{* *}=p$-value $<0.01,{ }^{* * *}=p$-value $<0.001$ (alpha risk $5 \%$ ). Scale bar $5 \mu \mathrm{m}$.

Figure 3. iqce morphants present ciliopathic phenotypes. (A-B). iqce morphants exhibit a curved body axis A: uninjected B: iqce-mo morphants, star indicates hydrocephalus. (C). Cysts in the paired pronephric glomeruli in iqce morphants (arrows). (D). Rescue of iqce-mo $(6 \mu \mathrm{M})$ injected embryos with different concentrations of iqce mRNA. $10 \mathrm{ng} / \mu \mathrm{l}(\mathrm{n}=121), 20 \mathrm{ng} / \mu \mathrm{l}(\mathrm{n}=163), 30 \mathrm{ng} / \mu \mathrm{l}$ $(n=193)$. The rescue was evaluated by the loss of curved body, data includes \pm SD only when 
needed (WT and the best condition (i.e. $30 \mathrm{ng} / \mu \mathrm{l})$ ). (E-G). Cilia in the pronephric duct of iqce morphants are misdirected, shortened, and malformed $(F)$ whereas in uninjected $(E)$ or control morpholinos (G) they are elongated. bar: $11.6 \mu \mathrm{m}$. (H-J). Cilia in nasal placode are reduced in number and size in morphants $(\mathrm{I}, \mathrm{n}=5)$ compared to uninjected $(\mathrm{H}, \mathrm{n}=5)$ and morpholino control $(\mathrm{J}, \mathrm{n}=5)$. bar: $11.6 \mu \mathrm{m}$. Scheme: Square shows the area of observation for pronephric cilia, and circle for nasal placode cilia. (K-L). $5 \mu \mathrm{M}$ sections through eyes of uninjected or iqce-mo injected embryos stained with toluidine blue (4 dpf). The uninjected embryos show normal lamination whereas morphants lack a properly formed retina. PRs: photoreceptors; ONL: outer nuclear layer; INL: inner nuclear layer; GCL: ganglion cell layer. Star indicates the outer segments of photoreceptors. Scale bar: $0.2 \mathrm{~cm}$. (M). Chart indicating the percentage of normal and situs inversus or heterotaxia in uninjected $(n=70)$, iqce-mo $(n=250)$ or iqce-mocont $(n=160)$ injected embryos, data includes \pm SD. (N-P). patched1 (ptc1) in situ hybridization revealed expanded expression in iqce morphant $(\mathrm{O}, \mathrm{n}=81)$ but not in uninjected $(\mathrm{N}, \mathrm{n}=88)$ or control morpholino $(\mathrm{P}$, $\mathrm{n}=30$ ) injected embryos. (Q-S). In situ immunohistochemistry showing expanded engrailed expression in iqce morphants $(\mathrm{R}, \mathrm{n}=10)$ compared to uninjected $(\mathrm{Q}, \mathrm{n}=10)$ and iqce-mocont $(\mathrm{S}$, $\mathrm{n}=10$ ) injected embryos. Scale bars: $47.2 \mu \mathrm{M}$. A-J: 48 hpf. K-M: 72 hpf. N-P: 10 somites. Q-S: 24 hpf. 


\section{Novel IQCE variations confirm its role in postaxial polydactyly and cause ciliary defect phenotype in zebrafish.}

Alejandro Estrada-Cuzcano ${ }^{1 *}$, Christelle Etard ${ }^{2 *}$, Clarisse Delvallée ${ }^{1 *}$, Corinne Stoetzel ${ }^{1}$, Elise Schaefer ${ }^{1,3}$, Sophie Scheidecker ${ }^{1,4}$, Véronique Geoffroy ${ }^{1}$, Aline Schneider ${ }^{1}$, Fouzia Studer ${ }^{5}$, Francesca Mattioli ${ }^{6}$, Kirsley Chennen ${ }^{1,7}$, Sabine Sigaudy8, Damien Plassard ${ }^{9}$, Olivier Poch ${ }^{7}$, Amélie Piton ${ }^{4,6}$, Uwe Strahle ${ }^{2}$, Jean Muller ${ }^{1,4 *}$ and Hélène Dollfus ${ }^{1,3,5 *}$

1. Laboratoire de Génétique médicale, UMR S INSERM U1112, IGMA, Faculté de Médecine FMTS, Université de Strasbourg, Strasbourg, France.

2. Karlsruhe Institute of Technology (KIT), Institute of Toxicology and Genetics (ITG), Eggenstein-Leopoldshafen, Germany.

3. Service de Génétique Médicale, Institut de Génétique Médicale d'Alsace, Hôpitaux Universitaires de Strasbourg, Strasbourg, France.

4. Laboratoires de Diagnostic Génétique, Hôpitaux Universitaires de Strasbourg, Strasbourg, France.

5. Centre de Référence pour les affections rares en génétique ophtalmologique, CARGO, Filière SENSGENE, Hôpitaux Universitaires de Strasbourg, 67091 Strasbourg, France.

6. Institut de Génétique et de Biologie Moléculaire et Cellulaire, 67400 Illkirch-Graffenstaden, France; INSERM U1258, 67400 Illkirch-Graffenstaden, France; CNRS UMR 7104, 67400 Illkirch-Graffenstaden, France; Université de Strasbourg, 67400 Illkirch, France.

7. Complex Systems and Translational Bioinformatics, ICube UMR 7357, Université de Strasbourg, Fédération de Médecine Translationnelle, Strasbourg, France.

8. Département de Génétique Médicale, Hôpital de la Timone, Marseille, France.

9. Plateforme GenomEast, IGBMC, Illkirch, France.

*equal contributor

Corresponding Author: Jean Muller/Hélène Dollfus

Email address: jeanmuller@unistra.fr / dollfus@unistra.fr 


\section{ABSTRACT}

Polydactyly is one of the most frequent inherited defects of the limbs characterized by supernumerary digits and high genetic heterogeneity. Among the many genes involved, either in isolated or syndromic forms, 8 have been implicated in postaxial polydactyly (PAP). Among those IQCE has been recently identified in a single consanguineous family. Using whole-exome sequencing in patients with uncharacterized ciliopathies including PAP, we identified 3 families with biallelic pathogenic variations in IQCE. Interestingly, the c.895_904del (p.Val301Serfs*8) was found in all families without sharing a common haplotype, suggesting a recurrent mechanism. Moreover, in 2 families, the systemic phenotype could be explained by additional pathogenic variants in known genes (TULP1, ATP6V1B1). RNA expression analysis on patients' fibroblasts confirms that dysfunction of IQCE leads to dysregulation of genes associated with the hedgehogsignaling pathway and zebrafish experiments demonstrate a full spectrum of phenotypes linked to defective cilia: body curvature, kidney cysts, left right asymmetry, misdirected cilia in pronephric duct and retinal defects. In conclusion, we identified 3 additional families confirming IQCE as a non-syndromic PAP gene. Our data emphasize, the importance of taking into account the complete set of variations of each individual as each clinical presentation could finally be explained by multiple genes.

Keywords: IQCE, cilia, polydactyly, hedgehog signaling, zebrafish, RNA-seq 


\section{INTRODUCTION}

Polydactyly is one of the most frequent inherited defects of the limb and has a prevalence of 0.33.6/1000 in live-births (Castilla et al., 1973; Malik, Ullah, Afzal, Lal, \& Haque, 2014). It is characterized by supernumerary digits, ranging from extra additive soft tissue without bone structure to an integral and fully developed digit (Umair, Ahmad, Bilal, Ahmad, \& Alfadhel, 2018). According to the position of the extra digit, polydactyly has been classified into postaxial (PAP), mesoaxial and preaxial (Malik, 2014). This defect is also associated with at least 221 syndromes according to the London Dysmorphology Database (Phadke \& Sankar, 2010) covering 146 genes (Umair et al., 2018) and can be a hallmark for a group of syndromes such as for ciliopathies. For this group of diseases, in addition to skeletal malformations such as PAP, the phenotypic spectrum can associate retinal degeneration, obesity, kidney dysfunction and sometimes intellectual disability as observed in the classical Bardet-Biedl syndrome (BBS; MIM\# 209900) for which 22 genes have been identified. In such heterogeneous diseases, high throughput genetic testing has made possible the diagnosis for many individuals (Shaheen et al., 2016) but also revealed more complex cases. Indeed, multi loci bearing pathogenic variations have been identified in several known disease related loci in single patients with apparent syndromic presentation (Karaca et al., 2018).

To date, eight genes FAM92A1, GLI1, GLI3, IQCE, KIAA0825, MIPOL1, PITX1 and ZNF141 (Ullah et al., 2019; Umair et al., 2018) have been implicated in non-syndromic polydactyly accounting for 286 disease causing variations according to HGMD (2018.1). Among those genes, IQCE (IQ domain-containing protein E) encodes a ciliary protein located at the base of the primary cilia and is linked to the Hedgehog $(\mathrm{Hh})$ signaling pathway (Pusapati et al., 2014). Hh signaling is one of many major pathways that controls key steps of embryonic development (Ingham, Nakano, \& Seger, 2011), tissue homeostasis and regeneration (Petrova \& Joyner, 2014) and related to human cancers (Barakat, Humke, \& Scott, 2010). Human hand and feet development is based on 
a highly conserved pentadactyl pattern (Abbasi, 2011), the conserved Hh signaling pathway regulates the precise digital shape and pattern in the limbs. IQCE together with EFCAB7 have an important regulatory role in the EvC complex (EVC-EVC2), a positive tissue regulator of $\mathrm{Hh}$ signaling. Recently, a homozygous splice acceptor variation in IQCE has been described in a single consanguineous family with PAP (Umair et al., 2017).

In our study, we report three families with biallelic pathogenic variations in IQCE identified by whole exome sequencing. Interestingly, these families with PAP were initially recruited as syndromic ciliopathies and two have additional pathogenic variations in other genes explaining their apparent syndromic phenotype. Functional studies based on the patient's cells or zebrafish (Danio rerio) assays confirm the ciliary role of IQCE.

\section{MATERIALS AND METHODS}

\section{Subjects}

The patients were recruited by the Strasbourg University Hospital Medical Genetics Department as well as by the Center for Rare Genetic Ophthalmologic Diseases (CARGO, Hôpitaux Universitaires de Strasbourg, Strasbourg) because they were suspected to have a ciliopathy. Study protocols used in each cohort have been approved by the corresponding Institutional Review Board or equivalent committees (in Strasbourg, "Comité de Protection des Personnes" EST IV, NDC-20142222), and written informed consent was given by each participant or parents. Our research complies with the Declaration of Helsinki. Written informed consent for open-access publication was provided by the participants or their parents. 


\section{Whole-exome sequencing}

Whole exome sequencing was performed by IntegraGen (Evry, France) for family A, B and C in 2011, 2013 and 2018 respectively. After shearing of the genomic DNA, library preparation was performed using either the Agilent Human All Exon Kits v2 (family A and B) or the Twist Human Core Exome Enrichment System (Twist Bioscience) and IntegraGen Custom additions (family C). Sequencing was performed on an Illumina HiSeq 2000/4000 to generate 75-bp paired-end reads following the manufacturer's protocols. Image analysis and base calling were performed using Illumina Real Time Analysis (1.14/2.7.7) with default parameters.

\section{Bioinformatics analysis}

The reads were mapped to the reference human genome (GRCh37/hg19) using BWA v0.7.12 (Etard, Roostalu, \& Strahle, 2010). GATK UG v3.4-46 was used to call SNV and indel variations (DePristo et al., 2011). Annotation and ranking of SNV and indel were performed by VaRank $\underline{1.4 .3}$ (Geoffroy et al., 2015) in combination with the Alamut Batch software $(1.11$, Interactive Biosoftware, Rouen, France). Very stringent filtering criteria were applied to filter out nonpathogenic variants (Supp. Table 1): (i) variants represented with an allele frequency of more than $1 \%$ in public variation databases including the 1000 Genomes (Altshuler et al., 2015), the gnomAD database (Lek et al., 2016), the DGV (MacDonald, Ziman, Yuen, Feuk, \& Scherer, 2014) or our internal exome database, (ii) variants in $5^{\prime}$ and $3^{\prime}$ UTR, downstream, upstream, intronic and synonymous locations without pathogenic prediction of local splice effect. Structural variants were predicted using CANOES (Backenroth et al., 2014) and annotated by AnnotSV 2.0 (Geoffroy, Herenger, et al., 2018). Our analysis considered all inheritance modes but given that 2 families were consanguineous we focused on compound heterozygous and homozygous variants consistent with a recessive transmission. Variations have been classified according to the ACMG 
guidelines (Richards et al., 2015) and only class 3 (likely pathogenic) and class 4 (pathogenic) have been considered.

\section{Homozygosity mapping}

Based on the WES data, homozygosity mapping has been performed using PLINK 1.9 (Chang et al., 2015). We followed the parameters described by Kancheva et al (Kancheva et al., 2016). The results are available in Supp. Table 2.

\section{Cell culture}

Skin fibroblasts from patient A.IV-1 and age-matched healthy control fibroblasts cells were grown in DMEM supplemented with $10 \%$ fetal calf serum (FCS) and 1\% PSG (penicillin-streptomycinglutamin). To induce primary cilium formation, the cells were deprived of serum by growth for $24 \mathrm{~h}$ in DMEM with $1 \%$ PSG but only $0.1 \%$ FCS. In order to activate the Hh pathway, the cells were incubated in a DMEM medium with 1\% PSG without serum (-FCS) and with SAG (100nM). SAG (Smoothened Agonist) has been resuspended in DMSO at a stock concentration of $4 \mathrm{mM}$.

\section{RNA extraction, cDNA synthesis, q-PCR and Taqman}

For RNA seq validation, cells have been cultured in normal condition DMEM 1\% PSG, 10\% FCS. Total RNA was prepared from patient A.IV-1 fibroblast pellets (technical triplicate) and three controls pellets (biological triplicate) using QIAshredder Kit (Qiagen Ref 79654) and RNeasy Kit (Qiagen Ref 74104) followed by a DNAse treatment with the TURBO DNA-free ${ }^{T M}$. Experiments have been repeated 3 times. For the Hh genes expression analysis (qRT-PCR), cells have been cultured in DMEM 1\% PSG (-FCS) + SAG (100nM). Total RNA was prepared as described above and experiments have been repeated twice with 2 different control pellets (biological duplicate) 
and one patient pellet (technical triplicate). RNA integrity was assessed by gel electrophoresis and RNA concentration by Eppendorf Biophotometer Plus with the Hellma® Tray Cell. Reverse transcription of $1 \mu \mathrm{g}$ total RNA to cDNA was performed using the BioRad iScript ${ }^{\mathrm{TM}}$ cDNA synthesis kit. Real-time quantitative polymerase chain reaction amplification was performed in a BioRad CFX96 TM Real-Time System using the $\mathrm{iQ}^{\mathrm{TM}}$ SYBR® Green Supermix and primer sets (Supp. Table 3) optimized for tested targets for SYBR Green-based real-time PCR for the real-time PCR. The normalized fold expression of the target gene was calculated using the comparative cycle threshold $\left(\mathrm{C}_{t}\right)$ method by normalizing target mRNA $\mathrm{C}_{t}$ to those for both GAPDH and HPRT reference gene using the CFX Manager Software Version 1.5 and excel calculation. Statistical analysis have been done using the "Graphpad prism" software.

\section{Immunocytochemistry and fluorescence microscopy}

A.IV-1 and control ciliated fibroblasts were seeded on $12 \mathrm{~mm}$ diameter cover slips. Ciliogenesis has been induced by $24 \mathrm{~h}$ incubation in a DMEM, 1\% PSG medium, without serum (-FCS). Then, cells have been fixed with PFA-PBS 4\%. Subsequently, the cells were stained with the ciliary markers anti-acetylated alpha tubulin (Lys40) mouse monoclonal primary antibody (1:200, \# 322700 Invitrogen) and anti-EVC2 rabbit polyclonal primary antibody (1:250, \# ab198930, Abcam). As secondary antibody, donkey anti-mouse Alexa 594 (1:1000, Invitrogen) and donkey anti-rabbit DyLight 488 (1:1000, \#611-741-127, Rockland - Tebu-bio) were used. Ciliated cells count between A.IV-1 and control was performed manually using $\sim 100$ cells per genotype (exact Fisher test was applied). Counts of EVC2 colocalization at the base of the cilia was performed manually using also 100 cells per genotype in triplicate (Student t-test has been applied). Pictures were taken with a Zeiss axio imager Z2 and the analysis with the ZEN2012 software (Carl Zeiss Inc., Oberkochen, Germany). 


\section{Transcriptome analysis}

RNA samples were extracted from two fibroblast replicates of A.IV-1 (A.IV-1.1 and A.IV-1.2), and replicates from 6 individuals affected by other ciliopathies or intellectual disabilities of known molecular origin (ARN1.1, ARN1.2, ARN2.1, ARN2.2, ARN3.1, ARN3.2, ARN4.1, ARN4.2, ARN5.1, ARN6.1, ARN6.2) using TRI reagent $₫$ (Molecular Research Center) or using the RNeasy Mini Kit Qiagen®. Both protocols included an additional step of DNAse I recombinant treatment (Sigma-Aldrich $®$ ). The integrity of the RNA was visualized on a $1 \%$ bleach agarose gel by electrophoresis (Aranda et al., 2012). Quantification and further quality analysis were performed using the Nanodrop $®$. Samples should have a 260/280 ratios around 2 and a 260/230 ratio above 1.7. The integrity and quality of the RNA were also evaluated by running samples on a RNA 6000 Nano Chip on the Bioanalyzer (Agilent Technologies) and samples should have a RNA integrity number (RIN) equal or above 8. Library preparation was performed at the GenomEast platform at the Institute of Genetics and Molecular and Cellular Biology (Strasbourg, France), using the TruSeq ${ }^{\circledR}$ stranded mRNA sample preparation kit (Illumina) starting from $1 \mu \mathrm{g}$ of extracted total RNA. Libraries were then $100+75 b p$ paired-end sequenced, with 2 samples per lane on an Illumina Hiseq4000 sequencer. The corresponding bioinformatics pipeline is described in detail in the supplementary information.

\section{Zebrafish stocks}

Fish were bred and raised at $28.5^{\circ} \mathrm{C}$ as described previously (Westerfield, 1993). The AB wildtype line (European Zebrafish Resource Centre (EZRC), Karlsruhe) was used for all the experiments. Zebrafish husbandry and experimental procedures were performed in accordance with German animal protection regulations (Regierungspräsidium Karlsruhe, Germany, AZ35- 
9185.81/G-137/10). Fish were crossed pairwise. For experiments, fertilized eggs were raised in 1× Instant Ocean salt solution (Aquarium Systems, Inc.) supplemented with $200 \mu$ M 1-phenyl 2thiourea (PTU) to suppress melanogenesis. For visualization of situs inversus, we used a transgenic line expressing GFP under the control of the skeletal and cardiac muscle specific promoter unc45b (Roostalu \& Strahle, 2012).

\section{In situ hybridization}

Whole-mount in situ hybridization was performed as previously described (Costa, Escaleira, Rodrigues, Manasfi, \& Mermelstein, 2002; Oxtoby \& Jowett, 1993). Ptc1 probe was obtained from (Concordet et al., 1996). wt1a probe was obtained from (Armant et al., 2013).

\section{Morpholinos and microinjections}

A search of the zebrafish genome (GRCz11) revealed a single orthologue of the human iqce gene (RefSeq: NM_001287204.1) encoding a protein sharing 58\% amino acid similarity with the human IQCE protein. Injections were performed as described previously (F. Muller et al., 1999). In brief, zebrafish eggs were collected shortly after being laid. Cleaned eggs were transferred to a petri dish with a minimal amount of water. Embryos were injected (FemtoJet; Eppendorf) through the chorion into the yolk at the one-cell stage. Injection needles were pulled from borosilicate glass capillary tubes with filament (Warner Instruments) using a micropipette puller (Sutter Instrument Co). Morpholinos were designed against the start codon of iqce (iqce-mo). Morpholino controls consist of the $5 \mathrm{bp}$ mismatch iqce-mo (iqce-mocont). Morpholinos (Gene Tools, LLC) were injected at the following concentrations: iqce-Mo: CAAGTTCTCCAGCTACCACAGACAT (0.6 $\mu \mathrm{M})$; iqcemocont; CAAcTTgTCgAGCTACCAgA cACAT $(0.6 \mu \mathrm{M})$. All dilutions were made in distilled water. Phenol red was added to the samples before injection ( $0.1 \%$ final concentration). Iqce-gfp plasmid was injected at the final concentration of $40 \mathrm{ng} / \mu \mathrm{l}$. When injected at $0.6 \mu \mathrm{M}$, neither the anti iqce 
nor the iqce-mocont (control) morpholino elicited necrosis, an unspecific effect of morpholino injections occasionally observed with some morpholinos (Robu et al., 2007). The efficiency of iqce-mo to block translation was assessed by co-injection of iqce-mo with a plasmid containing the iqce cDNA in-frame with gfp (iqce-gfp). As expected the embryos were depleted of GFP expression, indicating that the morpholinos indeed target efficiently iqce. In contrast, the 5mismatch iqce-mocont was unable to abrogate the translation of the fusion gene (Supp. Figure $3 A)$.

\section{PCR amplification}

Total RNA was isolated from 24 to $72 \mathrm{hpf}$ (hours post fertilization) embryos using Tri-reagent (Invitrogen, Carlsbad, CA). RT-PCR was carried out following standard protocols. For iqce in situ hybridization probe, a fragment of 580 bp was amplified by PCR with the following primers iqcewm5: GCTGCATCATGTAGGACGATG iqcewm3: CACTCTGAAGCTGACTCAAG, and cloned into pgem-t-easy vector (Promega). lqce full-length sequence was amplified from zebrafish cDNA with the following primers: iqce5: tcgGAATTCATGTCTGTGGTAGCTGGAGAAC; iqce3': agactcgagggTAAATCAAGTCA TCGGCACTG and cloned into pcs2+GFP with EcoRI-Xhol. For the rescue experiment iqce5'modif: tcgGAATTCATGTCGGTTGTAGCGGGCGAACTAG was used in combination with iqce3', and cloned into $p C S 2+G F P$ Morpholino-resistant iqce mRNA was synthesized with the SP6kit (Promega).

\section{Immunohistochemistry}

Fish embryos were fixed in 4\% paraformaldehyde/1x PBS one hour at room temperature. Embryos were permeabilized 7 min at $-20^{\circ} \mathrm{C}$ with cold acetone, rinsed 5 times, 5 min with $1 x$ PBS and blocked $4 \mathrm{~h}$ with BDP (1\% DMSO, 5\% BSA, 1x PBS). Incubation with primary antibody occurred 
over night at $4^{\circ} \mathrm{C}$, followed by thorough washing with PTW (1x PBS, $0.1 \%$ Tween 20$)$. Embryos were incubated with secondary antibody two hours at room temperature, and washed with PTW. Antibodies dilutions: Acetylated-tubulin (Sigma-Aldrich) 1/500; engrailed (Developmental Studies Hybridoma Bank, The University of lowa): 1/50; goat anti-rabbit alexa 488 (Invitrogen): 1/1000, goat anti-mouse alexa 488 (Invitrogen): 1/1000. For nucleus staining, we used Draq5 at 1/1000 dilution. Staining was analyzed by confocal microscopy (TCS SP2, Leica). For sectioning of the eyes, embryos were fixed in 4\% paraformaldehyde/PBS, progressively dehydrated in ethanol and stepwise transferred into Epon resin. Five $\mu \mathrm{m}$ sections were cut with a Leica microtome and stained with toluidine blue.

\section{RESULTS}

\section{Clinical features}

Three families with ciliopathy like phenotypes were referred to our laboratory as they remained unsolved following standard genetic testing. They all shared postaxial polydactyly (PAP) as a common feature but each presented other symptoms as described below (Figure 1A and Table 1). The affected patient of family A (A.IV-1) was born after an unremarkable pregnancy and is the only child of related Algerian parents. Bilateral polydactyly and brachydactyly of hands and feet were noted at birth associated with a syndactyly of the $2^{\text {nd }}$ and $3^{\text {rd }}$ digits of the feet. At the age of six years old, he was diagnosed with a decreased visual acuity and his ophthalmologic evaluation revealed retinal degeneration. He had hypogonadism and overweight was noted during his last clinical evaluation (15 years old). Family B is composed of three children presenting with PAP of hands and feet (bilateral for B.II-1 and unilateral for B.II-2 and B.II-3). The older one and the twin sister presented overweight respectively at the age of 7 and 5 years old in a context of obese parents. The ophthalmologic evaluation, including electroretinography, was normal for all the 
siblings. In family C, two affected children were reported. Individual C.IV-3 is the last child of related Moroccan parents and presented with PAP of both hands and one foot, learning disabilities and overweight. Her two sisters and parents share also the two last features. Her cousin, patient C.IV-4, born also from related parents is more severe. He had also PAP of the four limbs associated with renal anomalies, deafness and speech delay. The observed kidney disease consisted of a tubular nephropathy with nephrocalcinosis diagnosed at one year old evocative of a distal renal tubular acidosis. Deafness was detected at the age of two years old. His development was in the normal range, except for a language delay, in the context of hearing loss, which improved with speech therapy (Table 1).

The association of PAP and the other clinical features of each family member was assumed to be compatible with a syndromic ciliopathy (Wolff et al., 2004) and in particular to the Bardet-Biedl syndrome (BBS; MIM\# 209900). In line with the described phenotypes, the three families have been screened without success for the known BBS genes at that time (BBS1-BBS18) using either Sanger or targeted exome sequencing (J. Muller et al., 2010; Redin et al., 2012).

\section{Identification of IQCE pathogenic variations}

Whole exome sequencing (WES) was applied to all three families, generating on average a total of 81000 single nucleotide variants (SNVs), 11400 insertions-deletions (indels) and 21 structural variants (SV) (Supp. Table 1). In family A, as the proband (A.IV-1) was supposedly born from consanguineous parents, we identified 39 sizeable homozygous regions encompassing $238 \mathrm{Mb}$ (Supp. Table 2); supporting the assumption of consanguinity. In this case, autosomal recessive inheritance is more likely and we thus focused on homozygous variants, reducing the number of variants to 7 in 6 genes (DNAAF1, IQCE, MADD, SHANK2, TULP1 and ZDHHC2) (Supp. Table 1). A.IV-1 is affected by polydactyly, retinal dystrophy and hypogonadism, which made us, 
concentrate on 2 genes. The first one is IQCE (NM_152558.5), a gene that was just described as associated to PAP (Umair et al., 2017), with a homozygous 10 bp deletion in exon 12 c.895_904del (p.Val301Serfs ${ }^{\star}$ ) (Figure 1B) located in a conspicuous homozygous region of $2.4 \mathrm{Mb}$. This variant is present in gnomAD at a very low combined allele frequency $(0.0009534)$ with two homozygous carriers, which is compatible with the frequency of the disease. Subsequently, we analyzed IQCE exons 12 in 96 matched controls of Algerian ethnicity and could not find this variant (supplementary methods). Interestingly, the second gene is TULP1 (NM_001289395.1) a gene reported in Leber congenital amaurosis or retinitis pigmentosa (Hagstrom, North, Nishina, Berson, \& Dryja, 1998; Hanein et al., 2004) for which we identified a known homozygous pathogenic (class 5) variation, c.1198C>T (p.Arg400Trp), also located in a homozygous region of $8.5 \mathrm{Mb}$. This variant does not appear in public databases (gnomAD, 1000Genomes). The variants in both genes (IQCE and TULP1) segregated with the phenotypes within the family (Figure 1B) and in combination explained most of the clinical features of this individual. In the non-consanguineous family $\mathrm{B}$ (Figure 1A), WES revealed only 2 class-4 variations in IQCE at compound heterozygous state: c.895_904del (p.Val301Serfs*8) in exon 12 and c.1350_1353delAGAG, (p.Glu451Argfs*15) in exon 16 (Figure 1B). Both variations segregated in all affected members (B.II-1, B.II-2 and B.II3). The second variation (c.1350_1353delAGAG) was found in gnomAD with a frequency of 0.0002381 with 66 heterozygous carriers. In family C, WES analysis of the most severe case, C.IV-4 (Figure 1A), revealed a known homozygous variant in ATP6V1B1 (NM_001692.3), a gene that is associated with distal renal tubular acidosis with deafness (Vargas-Poussou et al., 2006) (dRTA; MIM\# 267300). This class-4 variant, c.175-1G>C, is located in a canonical splice site and is predicted to cause the skipping of exon 3, this variation was not found in public databases (gnomAD, 1000Genomes). Interestingly, the c.895_904del (p.Val301Serfs8) variation in IQCE that has been already observed in family A and B (respectively at the homozygous and compound heterozygous state) was only found in C.IV-4 at the heterozygous state (whereas no second pathogenic allele could be identified by cDNA sequencing and quantification, data not shown). 
The WES did not reveal any other variations in known PAP genes for this individual. Given that patient C.IV-3 had only PAP and given the C.IV-4 variation in IQCE, we sequenced the IQCE gene in C.IV-3 and surprisingly found the c.895_904del (p.Val301Serfs*8) at the homozygous state (Figure 1).

Given the recurrence of the c.895_904del (p.Val301Serfs $\left.{ }^{*} 8\right)$ variant in our cohort, we investigated a possible founder effect. Nevertheless, no common haplotype could be observed in the corresponding region in family $A$ and $B$ (Supp. Table 4). Analysis of the gnomAD record of this variation revealed its presence in different ethnical groups at a very low allele frequency: European (221/126532 alleles), Ashkenazi (12/10134 alleles), Latino (17/34392 alleles), African (6/23966 alleles), Finish (2/25788 alleles), south Asian (1/30782 alleles). The diverse origin of individuals with this IQCE variant and the differences in the haplotype suggested a recurrent mutational mechanism.

In order to better understand the effect of IQCE variants, we investigated the expression and subcellular localization of IQCE interacting partners in patient cells. IQCE is ubiquitously expressed in human adult tissues with higher levels of mRNA expression in lung, brain and hippocampus (Supp. Figure 1A). Endogenous IQCE is located at the base of the cilia and forms a complex with EFCAB7, to anchor the EVC-EVC2 sub-complex at the base of the cilia (EvC zone) for propagation of $\mathrm{Hh}$ signaling (Pusapati et al., 2014). We investigated the impact of IQCE deficiency in the formation of the primary cilia and localization of EVC-EVC2 sub-complex. Immunohistochemical studies on ciliated fibroblasts from individual A.IV-1 showed no differences in the number of ciliated cells (Supp. Figure 1B). Nevertheless, for $77 \%$ of patient's cells, we did not observe colocalization of EVC2 at the base of the cilia (Figure 2A and B), that presumably results in mislocalization of the EVC-EVC2 sub-complex and may compromise the Hh signaling.

\section{Transcriptome analysis and Hh signaling effect}


In order to get insight into the mechanistic impact of IQCE, we explored the transcriptome variation of individual A.IV-1 by RNA sequencing. We considered the possible impact of TULP1 variation. However, under physiological conditions, TULP1 is expressed exclusively in the retina, where it is involved in protein trafficking which is essential for the transport of rhodopsin (North, Naggert, Yan, Noben-Trauth, \& Nishina, 1997). This was confirmed as no TULP1 expression could be observed in the patient's fibroblasts. Given the IQCE genotype (frameshift) for A.IV-1 no IQCE expression could be detected. RNA differential expression analysis resulted in 98 significantly differentially expressed genes (Figure 2C and Supp. Table 5). None of the known IQCE interactors - EVC, EVC2, EFCAB7 and TCC23 (Breslow et al., 2018; Pusapati et al., 2018) - showed significant variations in their expression levels neither did the downstream effectors of the $\mathrm{Hh}$ signaling pathway GL/1, GL/2 and GL/3. We identified among the most significantly up and downregulated genes SLC35D3, SLC30A8 and GSTT1 respectively. SLC35D3 has been shown to be implicated in metabolic syndrome and obesity (Z. Zhang et al., 2014) and SLC30A8 is associated with a protective role against diabetes type2 (Flannick et al., 2014).

Regarding GSTT1, the protein catalyzes the conjugation of glutathione to a wide range of potential toxins as the first step in detoxification and is identified as risk factor for open angle glaucoma or ventricular diastolic dysfunction (Singh, Kumar, Tewari, \& Agarwal, 2018; Stamenkovic et al., 2018). In order to obtain functional clues about the IQCE protein modules, we applied functional enrichment analysis (WebGestalt 2019) (Wang, Vasaikar, Shi, Greer, \& Zhang, 2017); the overrepresentation analysis identified 10 significant clusters involving tissue development, ossification and skeletal system development among others (Figure 2D). A number of genes associated with Hh signaling and limb formation are included in these protein clusters (ALPL, ALDH1A1, FGF9, HOXC10, IGF2, IGFBP1, MECOM, MLLT3, NDP, SFRP1 and SMOC1) (Supp. Table 6). Selected genes with significant | log2 fold-change | > 0.5 were tested compared to controls by RT-qPCR and we confirmed the expression change of ALPL, GSTT1, IGF2, IQCE, MECOM, RSPO1 and SLC30A8 but we did not detect significant differences in FGF9 and SFRP1 (Figure 2E). 
In order to assess whether the Hh signaling is affected, we determined the expression levels of key genes in the patient's cells both under ciliary conditions and Hh activation (-FCS + SAG treatment). qRT-PCR revealed a significant under-expression of genes involved in the EVC-zone complex including EFCAB7, EVC, EVC2 and also GL/2 and GLI3 (Supp. Figure 2).

\section{Lack of iqce causes ciliary defects in zebrafish embryos}

Considering the ciliary localization of the human IQCE and encouraged by the previous studies, where genetic screens in zebrafish identified numerous defects of subpopulations of cilia (Zhao \& Malicki, 2007), we investigated expression and function of iqce in zebrafish (Danio rerio). We first assessed the iqce expression in the developing embryo. In situ hybridisation of iqce mRNA showed ubiquitous expression at the 10-somites and $20 \mathrm{hpf}$ stages with higher expression in the pronephric ducts at $20 \mathrm{hpf}$. At $48 \mathrm{hpf}$, higher levels of iqce mRNA were found in the pronephric ducts and in the mid- and hindbrain (Supp. Figure 3B).

A morpholino directed against the iqce start codon (iqce-mo) and a morpholino with a 5 mismatch control sequence (iqce-mocont) were used to elucidate the function of iqce in zebrafish embryos. Embryos injected with iqce-mo $(77 \%, \mathrm{n}=126)$ but not with the iqce-mocont control $(2 \%, \mathrm{n}=221)$ showed ventral curvature of the body axis (Figure 3A-B) which is often found in cilia mutants (Supp. Figure 3C). Occasionally we observed hydrocephalus (Figure 3B). In order to prove that the observed phenotype is due to the specific knock-down of iqce, we co-injected iqce-mo together with iqce mRNA resistant to iqce-mo. $54 \%$ of embryos exhibited a characteristic curved phenotype compared to $77 \%$ for iqce-mo alone (Figure 3D, $n=193$ ). We conclude from this experiment that the iqce-mo effectively abrogate the iqce expression. 
Bilaterally dilated kidney cyst were present in 37\% of iqce-mo morphants, but neither in uninjected nor in iqce-mocont injected embryos (Figure 3C and Supp. Figure 3D). These cysts are localized within the glomerulus as indicated by wt1a staining (Supp. Figure 3E).

We next examined the cilia of the pronephric duct. Embryos at $48 \mathrm{hpf}$ were stained with acetylatedtubulin antibody. In iqce-mocont and uninjected embryos, cilia are aligned along the duct forming a thin line (Figure 3E, G). In contrast, the cilia organization in iqce morphants was severely perturbed showing cilia oriented in all directions (Figure 3F). The misorientation and shortening of pronephric duct cilia can be due to dilation of the tubules and suggest that pronephric fluid flow may be affected (Zhao \& Malicki, 2007). We next tested whether other cilia are also affected, the dense ring of cilia on the epithelium surrounding the olfactory placode of 48-hpf wild-type larvae was strongly reduced in morphants (Figure $3 \mathrm{H}-\mathrm{J}$ ). In addition, the photoreceptor outer segment connects to the cell body via a narrow constriction that forms around a ciliary axoneme, and is known as the connecting cilium. Defects of photoreceptor cilia are associated with outer segment abnormalities and degeneration (Zhao \& Malicki, 2007). Therefore, we analyzed eye sections of 4 day old morphants. In non-injected embryos or control morphants all the layers are properly formed (Figure 3K). In contrast in iqce morphants, the lamination is disrupted and the retinal layer altered with no visible photoreceptor outer segment (Figure 3L).

The perturbation of motile cilia often results in a problem of left-right asymmetry. We thus examined the percentage of situs inversus in iqce morphants. iqce-mo or iqce-mocont were injected into embryos carrying a transgene that expresses GFP in the heart (-1.8 unc45b-TFP, (Roostalu \& Strahle, 2012). This allowed us to observe the looping of the heart. $44 \%$ of morphant embryos exhibited situs inversus (with the ventricle on the right and atrium on the left) or heterotaxia demonstrating that cilia from the Kupffer's vesicle, the fish equivalent of the mouse node, were affected by the iqce knock-down. In contrast only $3 \%$ and $6 \%$ of uninjected and iqcemocont injected embryos, respectively, showed reverse heart jogging (Figure 3M). 
In mutants with defective cilia (iguana) or ciliary motility (Irrc50), otoliths are frequently found ectopically, untethered or fused (Stooke-Vaughan, Huang, Hammond, Schier, \& Whitfield, 2012). We thus examined the number of otoliths in iqce morphants at $2 \mathrm{dpf}$. At $48 \mathrm{dpf}$ each ear should contains 2 otoliths and it is the case for $86 \%$ of the morphant. However, for $14 \%$ of them one of the two ears presented either one extra otolith ( 3 instead of 2$)$ or only one ( 1 instead of 2 ) (data not shown, $\mathrm{n}=262$ embryos).

Cilia are necessary for normal activation as well as repression of the hedgehog $(\mathrm{Hh})$ signaling pathway (Goetz \& Anderson, 2010; Huang \& Schier, 2009; Wolff et al., 2004). It was shown that in the absence of the ciliary protein BBS1 and under stimulation of Hh, the ciliary localization of Smoothened (Smo) was further enhanced compared to wild-type cells (Nozaki, Katoh, Kobayashi, \& Nakayama, 2018). We thus examined the expression of the Hh receptor Patch1 (Ptc1) and the downstream gene engrailed (eng) in the developing somites in iqce morphants. At 10 somites stages, transcription of ptc1 was expanded to the myotome, whereas it is exclusively restricted to the adaxial cells in control or uninjected embryos (Figure 3N-PO and Supp. Figure 3F). Similarly, the domain of eng expression was significantly expanded among the fast muscle fibres by $24 \mathrm{hpf}$, indicative of an increased number of Hh-dependent muscle fast fibers (Figure $3 Q-S$ ). These results indicate that the Hh pathway is perturbed by the loss of iqce function.

\section{DISCUSSION}

An IQCE homozygous variant affecting the splicing of the gene was recently identified by whole exome sequencing in a single Pakistani family presenting with postaxial polydactyly type A limited to lower limb (Umair et al., 2017). Here, we report 3 additional families with pathogenic variations in IQCE referred initially as ciliopathies with postaxial polydactyly involving the upper and lower limbs. Interestingly, the 3 families shared the same c.895_904del (p.Val301Serfs $\left.{ }^{*} 8\right)$ variation in 
IQCE exon 12 in a homozygous or in a heterozygous state with another truncating variant. These two IQCE variations have already been observed in the largest public databases. The c.1350_1353del (p.Glu451Argfs*15) was described in Exome Variant Server (EVS) (Tennessen et al., 2012) with an average allele frequency $>1.5 \%$ with 90 homozygous calls. Initially, this overestimated allele frequency prevented its identification as candidate pathogenic variation. The higher false positive rate for indel calls was a known issue solved while integrating in ExAC/gnomAD (0.024\% and no homozygous calls).

The index case A.IV-1 had a suspected ciliopathy-like phenotype, due to the presence of retinitis pigmentosa (RP), hypogonadism, polydactyly and speech delay. Although the proband was assumed as a patient with one syndrome, the occurrence of pathogenic variations in both IQCE and TULP1 can explain independently the polydactyly and the RP. In the family C, the phenotypes of the patients were different; C.IV-3 has only PAP and overweight compared to her cousin who has PAP, deafness and renal anomalies. Phenotypic variations in a single family is not unusual in ciliopathies even with a single gene involved (Abdelhamed et al., 2013; Gonzalez-Del Pozo et al., 2014; Katsanis et al., 2001; Ware, Aygun, \& Hildebrandt, 2011; W. Zhang et al., 2018). The variations in IQCE, can clearly explain the isolated PAP in C.IV-3. However, in the C.IV-4 individual, the association of deafness and nephropathy could finally be explained by the identification of variations in the ATP6V1B1 gene (dRTA; MIM\# 267300), known to be responsible for renal tubular acidosis associated with deafness. Nevertheless, the identification of only a single pathogenic allele in IQCE and the absence of any other variation in known polydactyly genes do not provide any explanation to date for the PAP for this patient. Although, gene dosage and RNA expression have been performed for this individual revealing still $50 \%$ of the IQCE expression level (data not shown), one cannot rule out missed variation(s) either in IQCE or in another gene. Whole genome sequencing might reveal intronic, intragenic or structural variations missed by the WES (Geoffroy, Stoetzel, et al., 2018). These two examples illustrate well how the incidental co- 
occurrence of 2 different conditions can mimic the clinical features of a known Mendelian disease (DeLuca, Weed, Haas, Halder, \& Stone, 2015; Karaca et al., 2018). This point out also the importance for searching for multiple hits in whole exome or whole genome sequencing data to explain some apparent syndromic phenotype such as ciliopathy-like phenotype. The occurrence of the other clinical manifestations, overweight or learning disability, in these families seemed to be due to other factors than the presence of neurosensory impairment (RP or deafness). However, we should take into consideration the appreciable variations of systemic features in individuals affected by pathogenic variants in different ciliopathy genes (Carmi, Elbedour, Stone, \& Sheffield, 1995; Estrada-Cuzcano et al., 2012; Waters \& Beales, 2011).

Vertebrate limb patterning is controlled by two signaling centers, the zone of polarizing activity (ZPA) and the distal posterior mesenchyme and the apical ectodermal ridge (AER) at the distal limb bud. The ZPA and AER produce signaling molecules -Hh and FGFs respectively- that control the formation of anteroposterior and proximodistal limb axes in a cooperative manner (Yamamoto et al., 2012). Additionally, Hh can induce a positive feedback Igf signaling during osteoblast differentiation (mostly lgf2), regulating in this way the long bone growth (Fisher, Meyer, Garber, \& Dealy, 2005; Shi, Chen, Karner, \& Long, 2015). Until recently, the combination of temporal and spatial exposure to long-range Hh signaling was accepted as the main molecular mechanism underlying digit patterning (Ahn \& Joyner, 2004; Harfe et al., 2004). However, current investigations in chicken limb buds show that mesenchymal cells in the posterior limb bud produce actin-based filopodia that accumulate particles of $\mathrm{Hh}$, allowing the $\mathrm{Hh}$ particles to act at a distance without extracellular diffusion (Sanders, Llagostera, \& Barna, 2013). This study suggested that proper activation of the Hh target genes is more critical for digit patterning than diffusible longrange $\mathrm{Hh}$ morphogen threshold. The polydactyly phenotype of other carriers of IQCE variations supports the involvement of IQCE in the formation of limbs, process that is related to Hh signaling. At the base of the cilia, the EvC complex (constituted by EVC-EVC2 and EFCAB7-IQCE) is not essential for Hh target gene induction. However, it can reduce the Hh-stimulated induction of GLI1 
or PTCH1 (Pusapati et al., 2014). Interestingly, gene ontology analysis of misregulated transcripts revealed enrichment for skeletal system development. Notably, IGF2 - a critical protein for bone formation (Fisher et al., 2005; Shi et al., 2015)- is misregulated in absence of IQCE. Defects in IGF2 in humans cause Silver-Russell syndrome, ectrodactyly, under masculinized genitalia, developmental delay, and placental hypoplasia (Begemann et al., 2015; Yamoto et al., 2017). Additionally, we observed the up-regulation of IGFBP1 and IGFBP3, these two proteins bind IGF2 and their excess may limit IGF2 function (Chao \& D'Amore, 2008).

Knockdown of iqce in zebrafish showed the full spectrum of phenotypes associated with defective cilia: body curvature, kidney cysts, left right asymmetry, misdirected cilia in pronephric duct (Shi, Su, Lipschutz, \& Lobo, 2017) as well as retinal defects resembling very much those observed in bbs9 morphants (Veleri et al., 2012). In addition, full iqce knockout mouse showed short tibias, thin retinas, metabolic and behavioural alterations as well as polydactyly. Hh expression during limb development is pivotal for defining the identity and number of digits. When its expression is activated at an ectopic site in the limb bud this define a pathogenic mechanism that leads to preaxial polydactyly (Lettice et al., 2012). The iqce zebrafish model exhibits modified Hh signalling. Indeed, we observed a broadening of the Hh ptc1 receptor outside of the adaxial cell, as well as extended Engrailed expression. It is well known that the primary cilium acts as a Hh signal transduction machine (Goetz, Ocbina, \& Anderson, 2009). Recently it was shown that in a bbs1 $\mathrm{KO}$ cell line, the entry of Smo into cilia upon Hh stimulation was increased compared to the wild type cell line (Nozaki et al., 2018). This in turn activated ectopic engrailed expression as observed in iqce zebrafish morphants. This result was unexpected since Pusapati et al. provided evidence by analysing osteoblasts and the NIH3T3 cell line that the EFCAB7-IQCE module anchors the EVC-EVC2 complex in a signalling microdomain at the base of the cilia. Moreover, they showed that EFCAB7 depletion triggered the mislocalization of EVC-EVC2 within cilia and impaired activation of the transcription factor GLI2. These data suggest an impairment of Hh signalling 
rather than the gain of $\mathrm{Hh}$ signalling activity in the iqce mutant background that we observed in the zebrafish. However, in zebrafish, no Evc or Evc2 orthologues have been identified but only a paralog absent in human (Pusapati et al., 2014). Transcriptome analysis of the patient's cells under normal condition did not provide evidences of GLI genes modified expression compared to controls. However, under Hh activation, the patient's cells show a reduced expression of most of the key Hh pathway genes (GLI2, GLI3, EFCAB7, EVC, EVC2) (Supp. Figure 2). At the protein level, we observed a significant amount of EVC2 mislocalization at the base of the cilia (Figure 2B) as well as a reduced GLI2 protein expression (data not shown).

Blindness is not present in patients with only iqce defect. However, it has been previously observed in other cases that $\mathrm{KO}$ of genes in zebrafish can give additional phenotypes not observed in patients. For example, the zebrafish cc2d2 adult mutants exhibit a scoliosis that is not observed in Joubert syndrome (JBTS; MIM\# 213300) (Bachmann-Gagescu et al., 2011). The defective retina of iqce morphants is very similar to the bbs 9 morphant model showing a lack of lamination (Veleri et al., 2012). This observation fits with the implication of iqce in ciliopathies.

In conclusion, our findings confirm the role of IQCE in non-syndromic postaxial polydactyly, and its negative impact on the Hh signaling pathway. The differences between iqce zebrafish and mice models compared to humans raise the question of additional genetic factors. This study illustrates how detailed and accurate clinical data are needed to interpret exome-scale genetic results and translate those results in correct counselling and therapeutic intervention. 


\section{AVAILABILITY OF DATA AND MATERIAL}

Data generated or analysed during this study are included in the published article and the corresponding supplementary data. The raw sequencing data generated in the course of this study are not publicly available due to the protocol and the corresponding consents used that did not include such information. All variants have been submitted to ClinVar using the following range of accessions numbers SCV000929968 to SCV000929972 (https://www.ncbi.nlm.nih.gov/clinvar/). Anonymised NGS data and genomic variant data files will be made available upon request from qualified investigators studying the molecular basis of genomic disorders. Datasets can be obtained via the corresponding author on reasonable request.

\section{ACKNOWLEDGMENTS}

We would like to thank the patient's family for their participation and the patient's associations, Formicoeur, Bardet-Biedl France and Unadev, for their constant and strong support. We also would like to acknowledge the members of the diagnostic laboratories (molecular and cytogenetic units, in particular Manuela Antin, Anne-Sophie Leuvrey and Elsa Nourisson) at the University Hospital in Strasbourg for technical assistance. We thank Nadine Borel and the zebrafish house team. We also thank our collaborators for screening their negative cohort (KM. Bujakowska, E. Pierce, P. Beales, F. Hildebrandt). We thank the GenomEast platform (Strasbourg, France) especially Serge Vicaire for library preparation, Céline Keime for supervising the transcriptome analysis as well as Bernard Jost and Christelle Thibault. The computing resources for this work were provided by the BICS and BISTRO bioinformatics platforms in Strasbourg.

\section{FUNDING INFORMATION}


Whole exome sequencing was funded by Retina France (100 exomes Program), by Hôpitaux Universitaires de Strasbourg (API 2013-2014 HUS $N^{\circ}$ 5885). RNASeq analysis performed in patients with IQCE pathogenic variant and other patients were funded by Fondation Maladie Rare and Fondation Jérome Lejeune. CE and US were supported by the HGF Biointerfaces Programme. AEC is supported by Fondation JED-Belgique. CD is supported by a FRM grant (ECO20170637509).

\section{CONFLICTS OF INTEREST}

The authors declare no conflict of interest. 


\section{REFERENCES}

Abbasi, A. A. (2011). Evolution of vertebrate appendicular structures: Insight from genetic and palaeontological data. Dev Dyn, 240(5), 1005-1016. doi:10.1002/dvdy.22572

Abdelhamed, Z. A., Wheway, G., Szymanska, K., Natarajan, S., Toomes, C., Inglehearn, C., \& Johnson, C. A. (2013). Variable expressivity of ciliopathy neurological phenotypes that encompass MeckelGruber syndrome and Joubert syndrome is caused by complex de-regulated ciliogenesis, Shh and Wnt signalling defects. Hum Mol Genet, 22(7), 1358-1372. doi:10.1093/hmg/dds546

Ahn, S., \& Joyner, A. L. (2004). Dynamic changes in the response of cells to positive hedgehog signaling during mouse limb patterning. Cell, 118(4), 505-516. doi:10.1016/j.cell.2004.07.023

Altshuler, D. M., Durbin, R. M., Abecasis, G. R., Bentley, D. R., Chakravarti, A., Clark, A. G., . . Consortium, G. P. (2015). A global reference for human genetic variation. Nature, 526(7571), 68-+. doi:10.1038/nature15393

Armant, O., Marz, M., Schmidt, R., Ferg, M., Diotel, N., Ertzer, R., . . Rastegar, S. (2013). Genome-wide, whole mount in situ analysis of transcriptional regulators in zebrafish embryos. Dev Biol, 380(2), 351-362. doi:10.1016/j.ydbio.2013.05.006

Bachmann-Gagescu, R., Phelps, I. G., Stearns, G., Link, B. A., Brockerhoff, S. E., Moens, C. B., \& Doherty, D. (2011). The ciliopathy gene cc2d2a controls zebrafish photoreceptor outer segment development through a role in Rab8-dependent vesicle trafficking. Hum Mol Genet, 20(20), 4041-4055. doi:10.1093/hmg/ddr332

Backenroth, D., Homsy, J., Murillo, L. R., Glessner, J., Lin, E., Brueckner, M., . . Shen, Y. F. (2014). CANOES: detecting rare copy number variants from whole exome sequencing data. Nucleic Acids Research, 42(12). doi:ARTN e97 10.1093/nar/gku345

Barakat, M. T., Humke, E. W., \& Scott, M. P. (2010). Learning from Jekyll to control Hyde: Hedgehog signaling in development and cancer. Trends Mol Med, 16(8), 337-348. doi:10.1016/j.molmed.2010.05.003

Begemann, M., Zirn, B., Santen, G., Wirthgen, E., Soellner, L., Buttel, H. M., . . Eggermann, T. (2015). Paternally Inherited IGF2 Mutation and Growth Restriction. N Engl J Med, 373(4), 349-356. doi:10.1056/NEJMoa1415227

Breslow, D. K., Hoogendoorn, S., Kopp, A. R., Morgens, D. W., Vu, B. K., Kennedy, M. C., ... Nachury, M. V. (2018). A CRISPR-based screen for Hedgehog signaling provides insights into ciliary function and ciliopathies. Nat Genet. doi:10.1038/s41588-018-0054-7

Carmi, R., Elbedour, K., Stone, E. M., \& Sheffield, V. C. (1995). Phenotypic differences among patients with Bardet-Biedl syndrome linked to three different chromosome loci. Am J Med Genet, 59(2), 199203. doi:10.1002/ajmg.1320590216

Castilla, E., Paz, J., Mutchinick, O., Munoz, E., Giorgiutti, E., \& Gelman, Z. (1973). Polydactyly: a genetic study in South America. Am J Hum Genet, 25(4), 405-412. Retrieved from http://www.ncbi.nlm.nih.gov/pubmed/4716659

Chang, C. C., Chow, C. C., Tellier, L. C., Vattikuti, S., Purcell, S. M., \& Lee, J. J. (2015). Second-generation PLINK: rising to the challenge of larger and richer datasets. Gigascience, 4, 7. doi:10.1186/s13742015-0047-8

Chao, W., \& D'Amore, P. A. (2008). IGF2: epigenetic regulation and role in development and disease. Cytokine Growth Factor Rev, 19(2), 111-120. doi:10.1016/j.cytogfr.2008.01.005

Concordet, J. P., Lewis, K. E., Moore, J. W., Goodrich, L. V., Johnson, R. L., Scott, M. P., \& Ingham, P. W. (1996). Spatial regulation of a zebrafish patched homologue reflects the roles of sonic hedgehog and protein kinase A in neural tube and somite patterning. Development, 122(9), 2835-2846. Retrieved from http://www.ncbi.nlm.nih.gov/pubmed/8787757 
Costa, M. L., Escaleira, R. C., Rodrigues, V. B., Manasfi, M., \& Mermelstein, C. S. (2002). Some distinctive features of zebrafish myogenesis based on unexpected distributions of the muscle cytoskeletal proteins actin, myosin, desmin, alpha-actinin, troponin and titin. Mechanisms of Development, 116(1-2), 95-104. doi:Pii S0925-4773(02)00149-1

Doi 10.1016/S0925-4773(02)00149-1

Deluca, A. P., Weed, M. C., Haas, C. M., Halder, J. A., \& Stone, E. M. (2015). Apparent Usher Syndrome Caused by the Combination of BBS1-Associated Retinitis Pigmentosa and SLC26A4-Associated Deafness. JAMA Ophthalmol, 133(8), 967-968. doi:10.1001/jamaophthalmol.2015.1463

DePristo, M. A., Banks, E., Poplin, R., Garimella, K. V., Maguire, J. R., Hartl, C., . . . Daly, M. J. (2011). A framework for variation discovery and genotyping using next-generation DNA sequencing data. Nat Genet, 43(5), 491-+. doi:10.1038/ng.806

Estrada-Cuzcano, A., Koenekoop, R. K., Senechal, A., De Baere, E. B., de Ravel, T., Banfi, S., ... Klevering, B. J. (2012). BBS1 mutations in a wide spectrum of phenotypes ranging from nonsyndromic retinitis pigmentosa to Bardet-Biedl syndrome. Arch Ophthalmol, 130(11), 1425-1432. doi:10.1001/archophthalmol.2012.2434

Etard, C., Roostalu, U., \& Strahle, U. (2010). Lack of Apobec2-related proteins causes a dystrophic muscle phenotype in zebrafish embryos. J Cell Biol, 189(3), 527-539. doi:10.1083/jcb.200912125

Fisher, M. C., Meyer, C., Garber, G., \& Dealy, C. N. (2005). Role of IGFBP2, IGF-I and IGF-II in regulating long bone growth. Bone, 37(6), 741-750. doi:10.1016/j.bone.2005.07.024

Flannick, J., Thorleifsson, G., Beer, N. L., Jacobs, S. B., Grarup, N., Burtt, N. P., . . Altshuler, D. (2014). Lossof-function mutations in SLC30A8 protect against type 2 diabetes. Nat Genet, 46(4), 357-363. doi:10.1038/ng.2915

Geoffroy, V., Herenger, Y., Kress, A., Stoetzel, C., Piton, A., Dollfus, H., \& Muller, J. (2018). AnnotSV: An integrated tool for Structural Variations annotation. Bioinformatics. doi:10.1093/bioinformatics/bty304

Geoffroy, V., Pizot, C., Redin, C., Piton, A., Vasli, N., Stoetzel, C., ... Muller, J. (2015). VaRank: a simple and powerful tool for ranking genetic variants. PeerJ, 3, e796. doi:10.7717/peerj.796

Geoffroy, V., Stoetzel, C., Scheidecker, S., Schaefer, E., Perrault, I., Bar, S., . . Muller, J. (2018). Wholegenome sequencing in patients with ciliopathies uncovers a novel recurrent tandem duplication in IFT140. Hum Mutat, 39(7), 983-992. doi:10.1002/humu.23539

Goetz, S. C., \& Anderson, K. V. (2010). The primary cilium: a signalling centre during vertebrate development. Nature Reviews Genetics, 11(5), 331-344. doi:10.1038/nrg2774

Goetz, S. C., Ocbina, P. J. R., \& Anderson, K. V. (2009). The Primary Cilium as a Hedgehog Signal Transduction Machine. Primary Cilia, 94, 199-+. doi:10.1016/S0091-679x(08)94010-3

Gonzalez-Del Pozo, M., Mendez-Vidal, C., Santoyo-Lopez, J., Vela-Boza, A., Bravo-Gil, N., Rueda, A., . . . Antinolo, G. (2014). Deciphering intrafamilial phenotypic variability by exome sequencing in a Bardet-Biedl family. Mol Genet Genomic Med, 2(2), 124-133. doi:10.1002/mgg3.50

Hagstrom, S. A., North, M. A., Nishina, P. L., Berson, E. L., \& Dryja, T. P. (1998). Recessive mutations in the gene encoding the tubby-like protein TULP1 in patients with retinitis pigmentosa. Nat Genet, 18(2), 174-176. doi:10.1038/ng0298-174

Hanein, S., Perrault, I., Gerber, S., Tanguy, G., Barbet, F., Ducroq, D., . . Kaplan, J. (2004). Leber congenital amaurosis: comprehensive survey of the genetic heterogeneity, refinement of the clinical definition, and genotype-phenotype correlations as a strategy for molecular diagnosis. Hum Mutat, 23(4), 306-317. doi:10.1002/humu.20010

Harfe, B. D., Scherz, P. J., Nissim, S., Tian, H., McMahon, A. P., \& Tabin, C. J. (2004). Evidence for an expansion-based temporal Shh gradient in specifying vertebrate digit identities. Cell, 118(4), 517528. doi:10.1016/j.cell.2004.07.024 
Huang, P., \& Schier, A. F. (2009). Dampened Hedgehog signaling but normal Wnt signaling in zebrafish without cilia. Development, 136(18), 3089-3098. doi:10.1242/dev.041343

Ingham, P. W., Nakano, Y., \& Seger, C. (2011). Mechanisms and functions of Hedgehog signalling across the metazoa. Nature Reviews Genetics, 12(6), 393-406. doi:10.1038/nrg2984

Kancheva, D., Atkinson, D., De Rijk, P., Zimon, M., Chamova, T., Mitev, V., . . Jordanova, A. (2016). Novel mutations in genes causing hereditary spastic paraplegia and Charcot-Marie-Tooth neuropathy identified by an optimized protocol for homozygosity mapping based on whole-exome sequencing. Genet Med, 18(6), 600-607. doi:10.1038/gim.2015.139

Karaca, E., Posey, J. E., Coban Akdemir, Z., Pehlivan, D., Harel, T., Jhangiani, S. N., . . Lupski, J. R. (2018). Phenotypic expansion illuminates multilocus pathogenic variation. Genet Med. doi:10.1038/gim.2018.33

Katsanis, N., Ansley, S. J., Badano, J. L., Eichers, E. R., Lewis, R. A., Hoskins, B. E., . . Lupski, J. R. (2001). Triallelic inheritance in Bardet-Biedl syndrome, a Mendelian recessive disorder. Science, 293(5538), 2256-2259. doi:10.1126/science.1063525

Lek, M., Karczewski, K. J., Minikel, E. V., Samocha, K. E., Banks, E., Fennell, T., . . Consortium, E. A. (2016). Analysis of protein-coding genetic variation in 60,706 humans. Nature, 536(7616), 285-+. doi:10.1038/nature19057

Lettice, L. A., Williamson, I., Wiltshire, J. H., Peluso, S., Devenney, P. S., Hill, A. E., . . Hill, R. E. (2012). Opposing functions of the ETS factor family define Shh spatial expression in limb buds and underlie polydactyly. Dev Cell, 22(2), 459-467. doi:10.1016/j.devcel.2011.12.010

MacDonald, J. R., Ziman, R., Yuen, R. K. C., Feuk, L., \& Scherer, S. W. (2014). The Database of Genomic Variants: a curated collection of structural variation in the human genome. Nucleic Acids Research, 42(D1), D986-D992. doi:10.1093/nar/gkt958

Malik, S. (2014). Polydactyly: phenotypes, genetics and classification. Clin Genet, 85(3), 203-212. doi:10.1111/cge.12276

Malik, S., Ullah, S., Afzal, M., Lal, K., \& Haque, S. (2014). Clinical and descriptive genetic study of polydactyly: a Pakistani experience of 313 cases. Clin Genet, 85(5), 482-486. doi:10.1111/cge.12217

Metsalu, T., \& Vilo, J. (2015). ClustVis: a web tool for visualizing clustering of multivariate data using Principal Component Analysis and heatmap. Nucleic Acids Research, 43(W1), W566-570. doi:10.1093/nar/gkv468

Muller, F., Blader, P., Rastegar, S., Fischer, N., Knochel, W., \& Strahle, U. (1999). Characterization of zebrafish smad1, smad2 and smad5: the amino-terminus of Smad1 and Smad5 is required for specific function in the embryo. Mechanisms of Development, 88(1), 73-88. doi:Doi 10.1016/S0925-4773(99)00173-2

Muller, J., Stoetzel, C., Vincent, M. C., Leitch, C. C., Laurier, V., Danse, J. M., . . . Dollfus, H. (2010). Identification of 28 novel mutations in the Bardet-Biedl syndrome genes: the burden of private mutations in an extensively heterogeneous disease. Hum Genet, 127(5), 583-593. doi:10.1007/s00439-010-0804-9

North, M. A., Naggert, J. K., Yan, Y., Noben-Trauth, K., \& Nishina, P. M. (1997). Molecular characterization of TUB, TULP1, and TULP2, members of the novel tubby gene family and their possible relation to ocular diseases. Proc Natl Acad Sci U S A, 94(7), 3128-3133. Retrieved from http://www.ncbi.nlm.nih.gov/pubmed/9096357

Nozaki, S., Katoh, Y., Kobayashi, T., \& Nakayama, K. (2018). BBS1 is involved in retrograde trafficking of ciliary GPCRs in the context of the BBSome complex. PLoS One, 13(3), e0195005. doi:10.1371/journal.pone.0195005 
Oxtoby, E., \& Jowett, T. (1993). Cloning of the Zebrafish Krox-20 Gene (Krx-20) and Its Expression during Hindbrain Development. Nucleic Acids Research, 21(5), 1087-1095. doi:DOI $10.1093 /$ nar/21.5.1087

Petrova, R., \& Joyner, A. L. (2014). Roles for Hedgehog signaling in adult organ homeostasis and repair. Development, 141(18), 3445-3457. doi:10.1242/dev.083691

Phadke, S. R., \& Sankar, V. H. (2010). Polydactyly and genes. Indian J Pediatr, 77(3), 277-281. doi:10.1007/s12098-010-0033-1

Pusapati, G. V., Hughes, C. E., Dorn, K. V., Zhang, D., Sugianto, P., Aravind, L., \& Rohatgi, R. (2014). EFCAB7 and IQCE regulate hedgehog signaling by tethering the EVC-EVC2 complex to the base of primary cilia. Dev Cell, 28(5), 483-496. doi:10.1016/j.devcel.2014.01.021

Pusapati, G. V., Kong, J. H., Patel, B. B., Krishnan, A., Sagner, A., Kinnebrew, M., . . Rohatgi, R. (2018). CRISPR Screens Uncover Genes that Regulate Target Cell Sensitivity to the Morphogen Sonic Hedgehog. Dev Cell, 44(2), 271. doi:10.1016/j.devcel.2018.01.002

Redin, C., Le Gras, S., Mhamdi, O., Geoffroy, V., Stoetzel, C., Vincent, M. C., . . Muller, J. (2012). Targeted high-throughput sequencing for diagnosis of genetically heterogeneous diseases: efficient mutation detection in Bardet-Biedl and Alstrom syndromes. J Med Genet, 49(8), 502-512. doi:10.1136/jmedgenet-2012-100875

Richards, S., Aziz, N., Bale, S., Bick, D., Das, S., Gastier-Foster, J., . . Committee, A. L. Q. A. (2015). Standards and guidelines for the interpretation of sequence variants: a joint consensus recommendation of the American College of Medical Genetics and Genomics and the Association for Molecular Pathology. Genet Med, 17(5), 405-424. doi:10.1038/gim.2015.30

Robu, M. E., Larson, J. D., Nasevicius, A., Beiraghi, S., Brenner, C., Farber, S. A., \& Ekker, S. C. (2007). p53 activation by knockdown technologies. PLoS Genet, 3(5), e78. doi:10.1371/journal.pgen.0030078

Roostalu, U., \& Strahle, U. (2012). In Vivo Imaging of Molecular Interactions at Damaged Sarcolemma. Dev Cell, 22(3), 515-529. doi:10.1016/j.devcel.2011.12.008

Sanders, T. A., Llagostera, E., \& Barna, M. (2013). Specialized filopodia direct long-range transport of SHH during vertebrate tissue patterning. Nature, 497(7451), 628-632. doi:10.1038/nature12157

Shaheen, R., Szymanska, K., Basu, B., Patel, N., Ewida, N., Faqeih, E., . . . Alkuraya, F. S. (2016). Characterizing the morbid genome of ciliopathies. Genome Biol, 17(1), 242. doi:10.1186/s13059016-1099-5

Shi, Y., Chen, J., Karner, C. M., \& Long, F. (2015). Hedgehog signaling activates a positive feedback mechanism involving insulin-like growth factors to induce osteoblast differentiation. Proc Natl Acad Sci U S A, 112(15), 4678-4683. doi:10.1073/pnas.1502301112

Shi, Y., Su, Y., Lipschutz, J. H., \& Lobo, G. P. (2017). Zebrafish as models to study ciliopathies of the eye and kidney. Clin Nephrol Res, 1(1), 6-9. Retrieved from http://www.ncbi.nlm.nih.gov/pubmed/29553143

Singh, M. M., Kumar, R., Tewari, S., \& Agarwal, S. (2018). Association of GSTT1/GSTM1 and ApoE variants with left ventricular diastolic dysfunction in thalassaemia major patients. Hematology, 1-6. doi:10.1080/10245332.2018.1502397

Stamenkovic, M., Lukic, V., Suvakov, S., Simic, T., Sencanic, I., Pljesa-Ercegovac, M., . . Djukic, T. (2018). GSTM1-null and GSTT1-active genotypes as risk determinants of primary open angle glaucoma among smokers. Int J Ophthalmol, 11(9), 1514-1520. doi:10.18240/ijo.2018.09.14

Stooke-Vaughan, G. A., Huang, P., Hammond, K. L., Schier, A. F., \& Whitfield, T. T. (2012). The role of hair cells, cilia and ciliary motility in otolith formation in the zebrafish otic vesicle. Development, 139(10), 1777-1787. doi:10.1242/dev.079947

Tennessen, J. A., Bigham, A. W., O'Connor, T. D., Fu, W., Kenny, E. E., Gravel, S., . . Project, N. E. S. (2012). Evolution and functional impact of rare coding variation from deep sequencing of human exomes. Science, 337(6090), 64-69. doi:10.1126/science.1219240 
Ullah, I., Kakar, N., Schrauwen, I., Hussain, S., Chakchouk, I., Liaqat, K., . . Leal, S. M. (2019). Variants in KIAA0825 underlie autosomal recessive postaxial polydactyly. Hum Genet. doi:10.1007/s00439019-02000-0

Umair, M., Ahmad, F., Bilal, M., Ahmad, W., \& Alfadhel, M. (2018). Clinical Genetics of Polydactyly: An Updated Review. Front Genet, 9, 447. doi:10.3389/fgene.2018.00447

Umair, M., Shah, K., Alhaddad, B., Haack, T. B., Graf, E., Strom, T. M., . . . Ahmad, W. (2017). Exome sequencing revealed a splice site variant in the IQCE gene underlying post-axial polydactyly type $A$ restricted to lower limb. Eur J Hum Genet, 25(8), 960-965. doi:10.1038/ejhg.2017.83

Vargas-Poussou, R., Houillier, P., Le Pottier, N., Strompf, L., Loirat, C., Baudouin, V., .. Blanchard, A. (2006). Genetic investigation of autosomal recessive distal renal tubular acidosis: evidence for early sensorineural hearing loss associated with mutations in the ATP6VOA4 gene. J Am Soc Nephrol, 17(5), 1437-1443. doi:10.1681/ASN.2005121305

Veleri, S., Bishop, K., Nogare, D. E. D., English, M. A., Foskett, T. J., Chitnis, A., . . Swaroop, A. (2012). Knockdown of Bardet-Biedl Syndrome Gene BBS9/PTHB1 Leads to Cilia Defects. PLoS One, 7(3). doi:ARTN e34389 10.1371/journal.pone.0034389

Wang, J., Vasaikar, S., Shi, Z., Greer, M., \& Zhang, B. (2017). WebGestalt 2017: a more comprehensive, powerful, flexible and interactive gene set enrichment analysis toolkit. Nucleic Acids Research, 45(W1), W130-W137. doi:10.1093/nar/gkx356

Ware, S. M., Aygun, M. G., \& Hildebrandt, F. (2011). Spectrum of clinical diseases caused by disorders of primary cilia. Proc Am Thorac Soc, 8(5), 444-450. doi:10.1513/pats.201103-025SD

Waters, A. M., \& Beales, P. L. (2011). Ciliopathies: an expanding disease spectrum. Pediatr Nephrol, 26(7), 1039-1056. doi:10.1007/s00467-010-1731-7

Westerfield, M. (1993). The zebrafish book: a guide for the laboratory use of zebrafish (Brachydanio reriro). 300.

Wolff, C., Roy, S., Lewis, K. E., Schauerte, H., Joerg-Rauch, G., Kirn, A., . . Ingham, P. W. (2004). iguana encodes a novel zinc-finger protein with coiled-coil domains essential for Hedgehog signal transduction in the zebrafish embryo. Genes \& Development, 18(13), 1565-1576. doi:10.1101/gad.296004

Yamamoto, M., Matsuzaki, T., Takahashi, R., Adachi, E., Maeda, Y., Yamaguchi, S., . . Noda, M. (2012). The transformation suppressor gene Reck is required for postaxial patterning in mouse forelimbs. Biol Open, 1(5), 458-466. doi:10.1242/bio.2012638

Yamoto, K., Saitsu, H., Nakagawa, N., Nakajima, H., Hasegawa, T., Fujisawa, Y., . . Ogata, T. (2017). De novo IGF2 mutation on the paternal allele in a patient with Silver-Russell syndrome and ectrodactyly. Hum Mutat, 38(8), 953-958. doi:10.1002/humu.23253

Zhang, W., Taylor, S. P., Ennis, H. A., Forlenza, K. N., Duran, I., Li, B., . . Cohn, D. H. (2018). Expanding the genetic architecture and phenotypic spectrum in the skeletal ciliopathies. Hum Mutat, 39(1), 152166. doi:10.1002/humu.23362

Zhang, Z., Hao, C. J., Li, C. G., Zang, D. J., Zhao, J., Li, X. N., . . Li, W. (2014). Mutation of SLC35D3 causes metabolic syndrome by impairing dopamine signaling in striatal D1 neurons. PLoS Genet, 10(2), e1004124. doi:10.1371/journal.pgen.1004124

Zhao, C. T., \& Malicki, J. (2007). Genetic defects of pronephric cilia in zebrafish. Mechanisms of Development, 124(7-8), 605-616. doi:10.1016/j.mod.2007.04.004 
$\begin{array}{lll}\text { Family A } & \text { Family B } & \text { Family C }\end{array}$

A.IV-1

B.II-1

B.II-2

B.II-3

C.IV-3

C.IV-4

\begin{tabular}{|c|c|c|c|c|c|c|}
\hline IQCE & $\begin{array}{l}\left.\text { p.[Val301Serfs }{ }^{*} 8\right] ; \\
\text { [Val301Serfs } 8]\end{array}$ & $\begin{array}{l}\text { p.[Val301Serfs*8]; } \\
\left.\text { [Glu451fs' }{ }^{*} 15\right]\end{array}$ & $\begin{array}{l}\text { p.[Val301Serfs*8]; } \\
\left.\text { [Glu451fs }{ }^{*} 15\right]\end{array}$ & $\begin{array}{l}\text { p.[Val301Serfs*8]; } \\
\left.\text { [Glu451fs }{ }^{*} 15\right]\end{array}$ & $\begin{array}{l}\text { p.[Val301Serfs*8]; } \\
\text { [Val301Serfs } 8]\end{array}$ & p.[Val301Serfs*8];[=] \\
\hline Other gene & $\begin{array}{c}\text { TULP1 } \\
\text { p.[Arg400Trp];[Arg400Trp] }\end{array}$ & & & & & $\begin{array}{c}\text { ATP6V1B1 } \\
\text { c. }[175-1 \mathrm{G}>\mathrm{C}] ;[175-1 \mathrm{G}>\mathrm{C}]\end{array}$ \\
\hline
\end{tabular}

\section{Clinical features}

Hands and feet abnormalities

Postaxial polydactyly

Hands

Feet

Brachydactyly

Syndactyly

Neurosensory disease

Retinal dystrophy

Deafness

$\mathrm{Y}$

Deafness

tural evaluation

Overweight

Development psychomotor

Intellectual abnormalities

Learning disabilities

Speech delay

Ataxia

Kidney function

Renal abnormality

Genital abnormalities

Hypogonadism

$\mathrm{Y}$
$\mathrm{Y}$
$\mathrm{Y}$
$\mathrm{Y}$
$\mathrm{Y}$

$\begin{array}{ll}Y & Y \\ Y & Y\end{array}$

$\begin{array}{ll}Y & Y \\ N & Y\end{array}$

$Y^{*}$
$Y^{*}$
$Y^{*}$
$Y$
$N$

$Y$
$Y^{*}$
$Y^{*}$
$Y$
$Y$

\begin{tabular}{ll} 
& \\
$Y$ & $Y$ \\
$Y$ & $Y$ \\
$N$ & $Y$ \\
$N$ & $N$ \\
& $N$ \\
\hline
\end{tabular}

$\mathrm{Y} \quad \mathrm{N}$

$\mathrm{N} \quad \mathrm{N}$

$\mathrm{N}$

$\mathrm{N}$

N

$\begin{array}{llllll} & & & \\ Y & Y & Y & N & N\end{array}$

$\mathrm{N} \quad \mathrm{N}$

$\mathrm{N}$
$\mathrm{N}$
$\mathrm{Y}$
$\mathrm{N}$

$\mathrm{N}$
$\mathrm{N}$
$\mathrm{N}$

$\mathrm{N}$
$\mathrm{N}$
$\mathrm{N}$
$\mathrm{N}$

$\mathrm{N}$

$N$
$N$

$\mathrm{N}$
$\mathrm{Y}$
$\mathrm{N}$

$\mathrm{N}$

$\mathrm{N} N$

$\mathrm{N}$

$\mathrm{N}$

N

N

N

$N$
$N$
$Y$
$N$

N

Table 1. Clinical characteristic of individuals with variants in IQCE. $\mathrm{Y}=\mathrm{yes}, \mathrm{N}=$ not present, ${ }^{*}=$ unilateral 
Figure 1. Clinical description and molecular genetic characterization of IQCE variations. (A) Graphic representation of three autosomal recessive families with complicated polydactyly in whom IQCE, TULP1 and ATP6V1B1 variations were identified. Sanger sequencing traces confirming the presence of the pathogenic variant and segregation in available family members is demonstrated. The "WT" symbol represents wild type and "M" symbol represents pathogenic variant. Blackened symbols indicate affected individuals. (B) Genomic structure (NC_000007.14) and schematic representation of the protein layout (NP_689771.3) of IQCE and location of currently known disease-associated variant (in bold, novel pathogenic variants identified in this study). Topology of IQCE represents one hydrophobic segment (HS), coil-coil conserved domain regions, three IQ calmodulin binding motifs (IQ) and one acid region (AR).

Figure 2. (A). Immunostaining of A.IV-1 and control ciliated fibroblasts, antibodies against human EVC2 (in green) and the ciliary marker anti acetylated alpha tubulin (in red), revealed that in absence of endogenous IQCE, EVC2 fails to localize at the base of the cilia. (B). Count of mislocalized EVC2 at the base of the cilia in fibroblasts under ciliated conditions (-FCS) between A.IV-1 and control (3 times $~ 100$ cells per condition). Statistical significance was determined using the Student's t-test, NS, non-significant, $\left.p=0.0004{ }^{(* * *}\right)$. (C). Heat map generated using ClustVis (Metsalu \& Vilo, 2015) from the transcriptome sequencing data reflecting gene counts of the significantly differentially expressed genes across patient and control samples. (D). Volcano plot of overrepresentation enrichment analysis of significantly misregulated transcripts in A.IV-1 fibroblast compared to wild type. These results were obtained using WebGestalt 2019, (Supp. Table 6). (E). Real-time quantitative polymerase chain reaction analysis from 3 technical replicates of A.IV-1 and 3 controls fibroblasts showed relative expression levels (normalized to GAPDH) of 9 significantly differentially expressed genes, data includes $\pm S D$. NS $=$ no significative, ${ }^{*}=p$-value $<0.05,{ }^{* *}=p$-value $<0.01,{ }^{* * *}=p$-value $<0.001$ (alpha risk $5 \%$ ). Scale bar $5 \mu \mathrm{m}$.

Figure 3. iqce morphants present ciliopathic phenotypes. (A-B). iqce morphants exhibit a curved body axis A: uninjected B: iqce-mo morphants, star indicates hydrocephalus. (C). Cysts in the paired pronephric glomeruli in iqce morphants (arrows). (D). Rescue of iqce-mo $(6 \mu \mathrm{M})$ injected embryos with different concentrations of iqce mRNA. $10 \mathrm{ng} / \mu \mathrm{l}(\mathrm{n}=121), 20 \mathrm{ng} / \mu \mathrm{l}(\mathrm{n}=163), 30 \mathrm{ng} / \mu \mathrm{l}$ $(n=193)$. The rescue was evaluated by the loss of curved body, data includes \pm SD only when 
needed (WT and the best condition (i.e. $30 \mathrm{ng} / \mu \mathrm{l})$ ). (E-G). Cilia in the pronephric duct of iqce morphants are misdirected, shortened, and malformed $(F)$ whereas in uninjected $(E)$ or control morpholinos (G) they are elongated. bar: $11.6 \mu \mathrm{m}$. (H-J). Cilia in nasal placode are reduced in number and size in morphants $(\mathrm{I}, \mathrm{n}=5)$ compared to uninjected $(\mathrm{H}, \mathrm{n}=5)$ and morpholino control $(\mathrm{J}, \mathrm{n}=5)$. bar: $11.6 \mu \mathrm{m}$. Scheme: Square shows the area of observation for pronephric cilia, and circle for nasal placode cilia. (K-L). $5 \mu \mathrm{M}$ sections through eyes of uninjected or iqce-mo injected embryos stained with toluidine blue (4 dpf). The uninjected embryos show normal lamination whereas morphants lack a properly formed retina. PRs: photoreceptors; ONL: outer nuclear layer; INL: inner nuclear layer; GCL: ganglion cell layer. Star indicates the outer segments of photoreceptors. Scale bar: $0.2 \mathrm{~cm}$. (M). Chart indicating the percentage of normal and situs inversus or heterotaxia in uninjected $(n=70)$, iqce-mo $(n=250)$ or iqce-mocont $(n=160)$ injected embryos, data includes \pm SD. (N-P). patched1 (ptc1) in situ hybridization revealed expanded expression in iqce morphant $(\mathrm{O}, \mathrm{n}=81)$ but not in uninjected $(\mathrm{N}, \mathrm{n}=88)$ or control morpholino $(\mathrm{P}$, $\mathrm{n}=30$ ) injected embryos. (Q-S). In situ immunohistochemistry showing expanded engrailed expression in iqce morphants $(\mathrm{R}, \mathrm{n}=10)$ compared to uninjected $(\mathrm{Q}, \mathrm{n}=10)$ and iqce-mocont $(\mathrm{S}$, $\mathrm{n}=10$ ) injected embryos. Scale bars: $47.2 \mu \mathrm{M}$. A-J: 48 hpf. K-M: 72 hpf. N-P: 10 somites. Q-S: 24 hpf. 


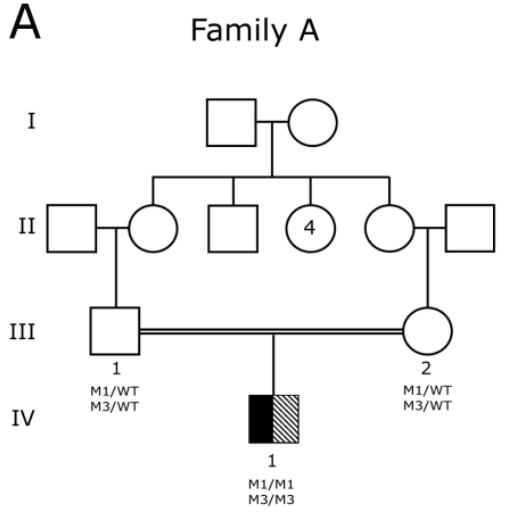

IV-1
M1

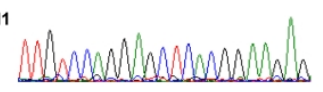

TGGCAAGCCTT TIGGCAGGAGC

M3

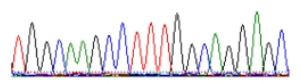

Family B

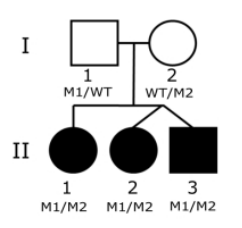

Family C

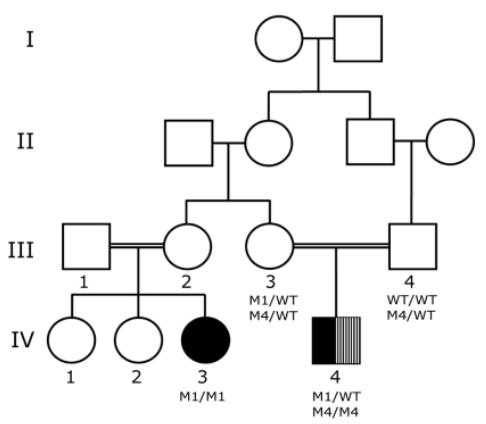

IV-3

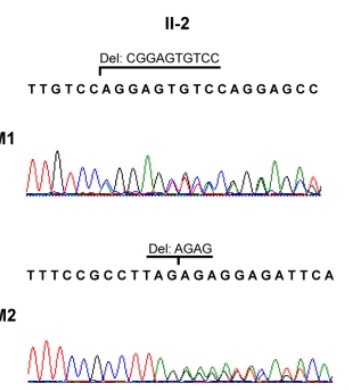
TTGTCCAGGAGCTCACGGAAGAG

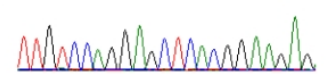

$$
\text { CccctcctgccaGtTtGCCCAGTA }
$$

M4

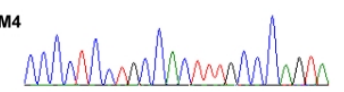

Polydactyly $\mathbb{E}$ Blindness $\quad$ Nephrocalcinosis and deafness

M1: IQCE c.895_904del; p.Val301Serfs*8 M2: IQCE c.1350_1353delAGAG; p. Glu451Argfs*15 M3: TULP1 c.1198C $>$ T; p.Arg400Trp M4: ATP6V1B1 C. $175-1 \mathrm{G}>\mathrm{C}$

B
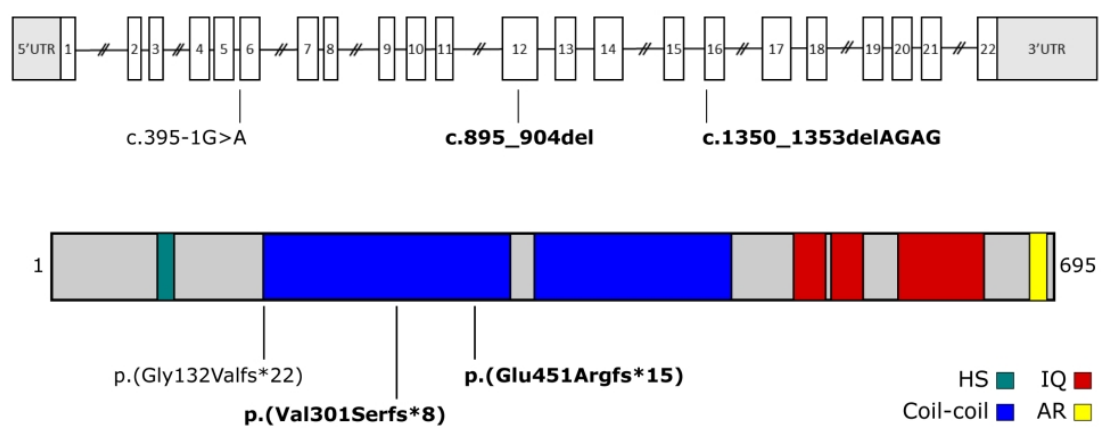

Figure 1. Clinical description and molecular genetic characterization of IQCE variations. (A) Graphic representation of three autosomal recessive families with complicated polydactyly in whom IQCE, TULP1 and ATP6V1B1 variations were identified. Sanger sequencing traces confirming the presence of the pathogenic variant and segregation in available family members is demonstrated. The "WT" symbol represents wild type and " $M$ " symbol represents pathogenic variant. Blackened symbols indicate affected individuals. (B) Genomic structure (NC_000007.14) and schematic representation of the protein layout (NP_689771.3) of IQCE and location of currently known disease-associated variant (in bold, pathogenic variants identified in this study). Topology of IQCE represents one hydrophobic segment (HS), coil-coil conserved domain regions, three IQ calmodulin binding motifs (IQ) and one acid region (AR). 
A
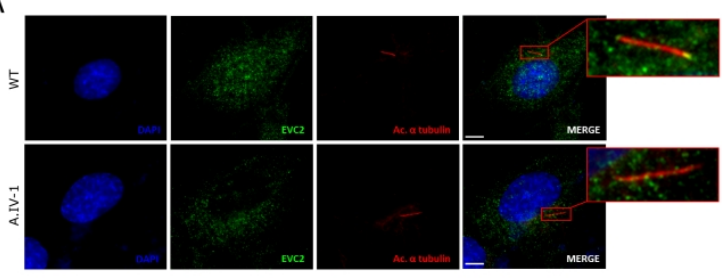

C

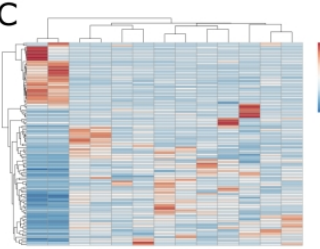

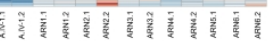

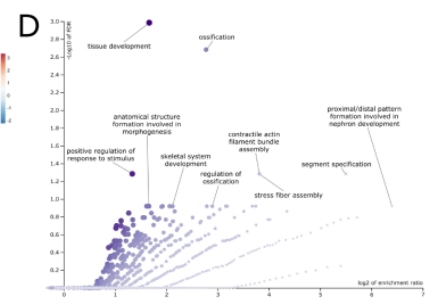

B

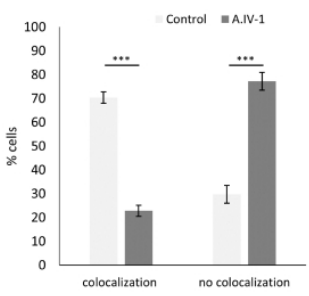

$E$

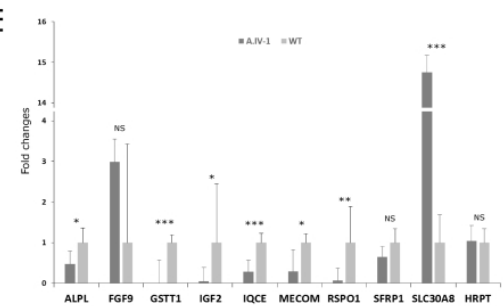

Figure 2. (A). Immunostaining of A.IV-1 and control ciliated fibroblasts, antibodies against human EVC2 (in green) and the ciliary marker anti acetylated alpha tubulin (in red), revealed that in absence of endogenous IQCE, EVC2 fails to localize at the base of the cilia. (B). Count of mislocalized EVC2 at the base of the cilia in fibroblasts under ciliated conditions (-FCS) between A.IV-1 and control ( 3 times 100 cells per condition). Statistical significance was determined using the Student's t-test, NS, non-significant, $\mathrm{p}=0.0004(* * *)$. (C). Heat map generated using ClustVis (Metsalu \& Vilo, 2015) from the transcriptome sequencing data reflecting gene counts of the significantly differentially expressed genes across patient and control samples. (D).

Volcano plot of overrepresentation enrichment analysis of significantly misregulated transcripts in A.IV-1 fibroblast compared to wild type. These results were obtained using WebGestalt 2019, (Supp. Table 6). (E).

Real-time quantitative polymerase chain reaction analysis from 3 technical replicates of A.IV-1 and 3 controls fibroblasts showed relative expression levels (normalized to GAPDH) of 9 significantly differentially expressed genes, data includes $\pm S D$. NS $=$ no significative, $*=p$-value $<0.05, * *=p$-value $<0.01, * * *=p-$ value $<0.001$ (alpha risk $5 \%$ ). Scale bar $5 \mu \mathrm{m}$. 

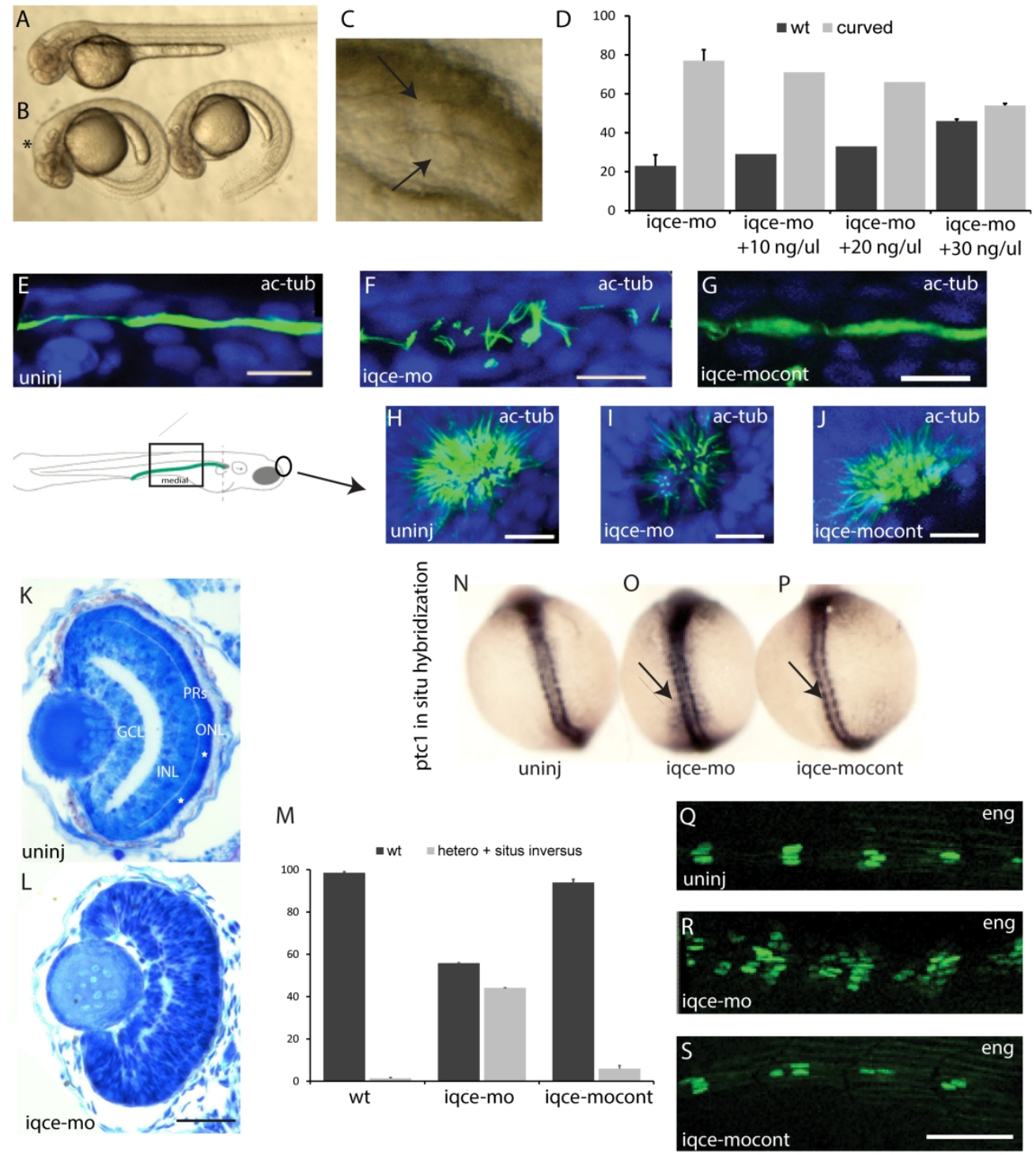

Figure 3. iqce morphants present ciliopathic phenotypes. (A-B). iqce morphants exhibit a curved body axis A: uninjected B: iqce-mo morphants, star indicates hydrocephalus. (C). Cysts in the paired pronephric glomeruli in iqce morphants (arrows). (D). Rescue of iqce-mo $(6 \mu \mathrm{M})$ injected embryos with different concentrations of iqce mRNA. $10 \mathrm{ng} / \mu \mathrm{l}(\mathrm{n}=121), 20 \mathrm{ng} / \mu \mathrm{l}(\mathrm{n}=163), 30 \mathrm{ng} / \mu \mathrm{l}(\mathrm{n}=193)$. The rescue was evaluated by the loss of curved body, data includes \pm SD only when needed (WT and the best condition (i.e. $30 \mathrm{ng} / \mu \mathrm{l}))$. (E-G). Cilia in the pronephric duct of iqce morphants are misdirected, shortened, and malformed

(F) whereas in uninjected (E) or control morpholinos (G) they are elongated. bar: $11.6 \mu \mathrm{m}$. $(\mathrm{H}-\mathrm{J})$. Cilia in nasal placode are reduced in number and size in morphants $(I, n=5)$ compared to uninjected $(H, n=5)$ and morpholino control $(\mathrm{J}, \mathrm{n}=5)$. bar: $11.6 \mu \mathrm{m}$. Scheme: Square shows the area of observation for pronephric cilia, and circle for nasal placode cilia. (K-L). $5 \mu \mathrm{M}$ sections through eyes of uninjected or iqce-mo injected embryos stained with toluidine blue (4 dpf). The uninjected embryos show normal lamination whereas morphants lack a properly formed retina. PRs: photoreceptors; ONL: outer nuclear layer; INL: inner nuclear layer; GCL: ganglion cell layer. Star indicates the outer segments of photoreceptors. Scale bar: $0.2 \mathrm{~cm}$. (M). Chart indicating the percentage of normal and situs inversus or heterotaxia in uninjected $(n=70)$, iqce-mo $(n=250)$ or iqce-mocont $(n=160)$ injected embryos, data includes \pm SD. (N-P). patched1 (ptc1) in situ hybridization revealed expanded expression in iqce morphant $(\mathrm{O}, \mathrm{n}=81)$ but not in uninjected $(\mathrm{N}, \mathrm{n}=88)$ or 
control morpholino $(P, n=30)$ injected embryos. (Q-S). In situ immunohistochemistry showing expanded engrailed expression in iqce morphants $(R, n=10)$ compared to uninjected $(Q, n=10)$ and iqce-mocont $(S$, $\mathrm{n}=10$ ) injected embryos. Scale bars: $47.2 \mu \mathrm{M}$. A-J: 48 hpf. K-M: 72 hpf. N-P: 10 somites. Q-S: 24 hpf. 


\begin{tabular}{|c|c|c|c|c|c|c|}
\hline & \multirow{2}{*}{$\begin{array}{c}\text { Family A } \\
\text { A.IV-1 }\end{array}$} & \multicolumn{3}{|c|}{ Family B } & \multicolumn{2}{|c|}{ Family C } \\
\hline & & B.Il-1 & B.II-2 & B.II-3 & C.IV-3 & C.IV-4 \\
\hline IQCE & $\begin{array}{l}\text { p.[Val301Serfs*8]; } \\
\text { [Val301Serfs } 8 \text {; } 8 \text {; }\end{array}$ & $\begin{array}{l}\text { p.[Val301Serfs*8]; } \\
\text { [Glu451fs' } 15]\end{array}$ & $\begin{array}{l}\text { p.[Val301Serfs* } 8] ; \\
\left.\text { [Glu4511fs }{ }^{*} 15\right]\end{array}$ & $\begin{array}{l}\text { p.[Val301Serfs*8]; } \\
\left.\text { [Glu451fs }{ }^{*} 15\right]\end{array}$ & $\begin{array}{l}\text { p.[Val301Serfs*8]; } \\
\text { [Val301Serfs" }{ }^{\star} \text { ] }\end{array}$ & p.[Val301Serfs"8];[=] \\
\hline Other gene & $\begin{array}{c}\text { TULP1 } \\
\text { p.[Arg400Trp];[Arg400Trp] }\end{array}$ & & & & & $\begin{array}{c}\text { ATP6V1B1 } \\
\text { c. }[175-1 \mathrm{G}>\mathrm{C}] ;[175-1 \mathrm{G}>\mathrm{C}]\end{array}$ \\
\hline \multicolumn{7}{|l|}{ Clinical features } \\
\hline \multicolumn{7}{|c|}{ Hands and feet abnormalities } \\
\hline Postaxial polydactyly & $\mathrm{Y}$ & $\mathrm{Y}$ & $\mathrm{Y}$ & $\mathrm{Y}$ & $\mathrm{Y}$ & $\mathrm{Y}$ \\
\hline Hands & Y & Y & $Y^{*}$ & $Y^{*}$ & Y & Y \\
\hline Feet & $\mathrm{Y}$ & $\mathrm{Y}$ & $Y^{*}$ & $Y^{*}$ & $\mathrm{Y}$ & Y \\
\hline Brachydactyly & Y & Y & $\mathrm{Y}$ & $\mathrm{Y}$ & $\mathrm{N}$ & $\mathrm{N}$ \\
\hline Syndactyly & Y & $\mathrm{N}$ & $\mathrm{N}$ & Y & $\mathrm{N}$ & $\mathrm{N}$ \\
\hline \multicolumn{7}{|l|}{ Neurosensory disease } \\
\hline Retinal dystrophy & $\mathrm{Y}$ & $\mathrm{N}$ & $\mathrm{N}$ & $\mathrm{N}$ & $\mathrm{N}$ & $\mathrm{N}$ \\
\hline Deafness & $\mathrm{N}$ & $\mathrm{N}$ & $\mathrm{N}$ & $\mathrm{N}$ & $\mathrm{N}$ & $\mathrm{Y}$ \\
\hline \multicolumn{7}{|l|}{ Pondostatural evaluation } \\
\hline Overweight & $\mathrm{Y}$ & $\mathrm{Y}$ & $\mathrm{Y}$ & $\mathrm{N}$ & $\mathrm{Y}$ & $\mathrm{N}$ \\
\hline \multicolumn{7}{|c|}{ Development psychomotor } \\
\hline Intellectual abnormalities & $\mathrm{N}$ & $\mathrm{N}$ & $\mathrm{N}$ & $\mathrm{N}$ & $\mathrm{N}$ & $\mathrm{N}$ \\
\hline Learning disabilities & $\mathrm{N}$ & $\mathrm{N}$ & $\mathrm{N}$ & $\mathrm{N}$ & $\mathrm{Y}$ & $\mathrm{N}$ \\
\hline Speech delay & $\mathrm{Y}$ & $\mathrm{N}$ & $\mathrm{N}$ & $\mathrm{N}$ & Y & $\mathrm{Y}$ \\
\hline Ataxia & $\mathrm{N}$ & $\mathrm{N}$ & $\mathrm{N}$ & $\mathrm{N}$ & $\mathrm{N}$ & $\mathrm{N}$ \\
\hline \multicolumn{7}{|l|}{ Kidney function } \\
\hline Renal abnormality & $\mathrm{N}$ & $\mathrm{N}$ & $\mathrm{N}$ & $\mathrm{N}$ & $\mathrm{N}$ & $\begin{array}{l}\text { Tubulopathy - } \\
\text { Nephrocalcinosis }\end{array}$ \\
\hline \multicolumn{7}{|l|}{ Genital abnormalities } \\
\hline Hypogonadism & Micropenis & $\mathrm{N}$ & & & $\mathrm{N}$ & $\mathrm{N}$ \\
\hline
\end{tabular}

Table 1. Clinical characteristic of individuals with variants in IQCE. $\mathrm{Y}=\mathrm{yes}, \mathrm{N}=$ not present, ${ }^{*}=$ unilateral 
$\begin{array}{lll}\text { Family A } & \text { Family B } & \text { Family C }\end{array}$

A.IV-1

B.II-1

B.II-2

B.II-3

C.IV-3

C.IV-4

\begin{tabular}{|c|c|c|c|c|c|c|}
\hline IQCE & $\begin{array}{l}\left.\text { p.[Val301Serfs }{ }^{*} 8\right] ; \\
\text { [Val301Serfs } 8]\end{array}$ & $\begin{array}{l}\text { p.[Val301Serfs*8]; } \\
\left.\text { [Glu451fs' }{ }^{*} 15\right]\end{array}$ & $\begin{array}{l}\text { p.[Val301Serfs*8]; } \\
\left.\text { [Glu451fs }{ }^{*} 15\right]\end{array}$ & $\begin{array}{l}\text { p.[Val301Serfs*8]; } \\
\left.\text { [Glu451fs }{ }^{*} 15\right]\end{array}$ & $\begin{array}{l}\text { p.[Val301Serfs*8]; } \\
\text { [Val301Serfs } 8]\end{array}$ & p.[Val301Serfs*8];[=] \\
\hline Other gene & $\begin{array}{c}\text { TULP1 } \\
\text { p.[Arg400Trp];[Arg400Trp] }\end{array}$ & & & & & $\begin{array}{c}\text { ATP6V1B1 } \\
\text { c. }[175-1 \mathrm{G}>\mathrm{C}] ;[175-1 \mathrm{G}>\mathrm{C}]\end{array}$ \\
\hline
\end{tabular}

\section{Clinical features}

Hands and feet abnormalities

Postaxial polydactyly

Hands

Feet

Brachydactyly

Syndactyly

Neurosensory disease

Retinal dystrophy

Deafness

$\mathrm{Y}$

Deafness

tural evaluation

Overweight

Development psychomotor

Intellectual abnormalities

Learning disabilities

Speech delay

Ataxia

Kidney function

Renal abnormality

Genital abnormalities

Hypogonadism

$\mathrm{Y}$
$\mathrm{Y}$
$\mathrm{Y}$
$\mathrm{Y}$
$\mathrm{Y}$

$\begin{array}{ll}Y & Y \\ Y & Y\end{array}$

$\begin{array}{ll}Y & Y \\ N & Y\end{array}$

$Y^{*}$
$Y^{*}$
$Y^{*}$
$Y$
$N$

$Y$
$Y^{*}$
$Y^{*}$
$Y$
$Y$

\begin{tabular}{ll} 
& \\
$Y$ & $Y$ \\
$Y$ & $Y$ \\
$N$ & $Y$ \\
$N$ & $N$ \\
& $N$ \\
\hline
\end{tabular}

$\mathrm{Y} \quad \mathrm{N}$

$\mathrm{N} \quad \mathrm{N}$

$\mathrm{N}$

$\mathrm{N}$

N

$\begin{array}{llllll} & & & \\ Y & Y & Y & N & N\end{array}$

$\mathrm{N} \quad \mathrm{N}$

$\mathrm{N}$
$\mathrm{N}$
$\mathrm{Y}$
$\mathrm{N}$

$\mathrm{N}$
$\mathrm{N}$
$\mathrm{N}$

$\mathrm{N}$
$\mathrm{N}$
$\mathrm{N}$

$\mathrm{N}$

$N$
$N$

$\mathrm{N}$
$\mathrm{Y}$
$\mathrm{N}$

$\mathrm{N}$

$\mathrm{N} N$

$\mathrm{N}$

$\mathrm{N}$

N

N

N

$N$
$N$
$Y$
$N$

N

Table 1. Clinical characteristic of individuals with variants in IQCE. $\mathrm{Y}=\mathrm{yes}, \mathrm{N}=$ not present, ${ }^{*}=$ unilateral 


\section{SUPPLEMENTARY INFORMATION}

\section{Sanger Sequencing}

Sanger sequencing was performed by way of PCR amplification with 50ng of genomic DNA template. The primers were designed with Primer 3 (http://frodo.wi.mit.edu/primer3). Bidirectional sequencing of the purified PCR products was performed by Eurofins Genomics Sequencing Facilities (https://www.eurofinsgenomics.eu/en/custom-dna-sequencing).

\section{Control screening}

IQCE exon 12 was tested in 93 control DNA of matched origin (Algerian) and 93 multi ethnic. High resolution melting (HRM) assay was performed in a $20 \mu$ reaction volume containing 50 ng of template DNA, $15 \mu$ Precision Melt Supermix (Bio-Rad) and $10 \mu \mathrm{mol}$ of each primer of the primer set. PCR was performed using a CFX96 (Bio-Rad) thermal cycler and consisted of predenaturation $\left(98^{\circ} \mathrm{C}\right.$ for $2 \mathrm{~min}$ ) followed by 35 cycles of amplification involving denaturation $\left(98^{\circ} \mathrm{C}\right.$ for $\left.5 \mathrm{~s}\right)$, annealing $\left(60^{\circ} \mathrm{C}\right.$ for $\left.10 \mathrm{~s}\right)$ and a melting curve involving denaturation $\left(95^{\circ} \mathrm{C}\right.$ for $30 \mathrm{~s})$, annealing $\left(70^{\circ} \mathrm{C}\right.$ for $\left.30 \mathrm{~s}\right)$ and a melt curve $\left(72-95^{\circ} \mathrm{C}\right.$ with an increment of $0.2^{\circ} \mathrm{C}$ per 10 s). Data generated were analyzed using Precision Melt Analysis software (Bio-Rad). In HRM analysis, differences in Tm and normalized curve shape were used together to discriminate even the most difficult-to-detect sequence variation.

\section{Bioinformatic pipeline RNA seq}

Image analysis and base calling were performed using RTA v.2.7.3 and blc2fastq v.2.17.1.14 (Illumina). Sequence reads were mapped onto hg19/GRCh37 assembly of human genome using Tophat 2.0.14 (Kim et al., 2013) and bowtie version 2-2.1.0 (Langmead, Trapnell, Pop, \& Salzberg, 2009), A summary of the mapping results is available in Supplementary Table 7. Gene expression was quantified from uniquely aligned reads using HTSeq-0.6.1 with gene annotations from Ensembl release 75 and intersection non-empty mode.

\section{Differential expression RNA-seq}

In order to identify variation in mRNA expression due to the absence of IQCE in the patient cells, comparisons of read counts were performed using $\mathrm{R} 3.2 .5$ with the statistical method proposed by Anders and Huber implemented in the DESeq2 v.1.10.1 Bioconductor package 
(Love, Huber, \& Anders, 2014). The generated data were explored and visualized to assess and check data quality and to eventually remove bad quality data. Especially, PCA was built after stabilizing variance using the regularized log transformation method or RSeqQC was used to do some quality controls such as gene body coverage. Only genes with | log2 foldchange $\mid>0.5$ were considered, as we expected to identify a large amount of change between transcriptomic results from one patient compared to other individuals (indirect consequences of the pathogenic mutation or unrelated events due to variability between humans). To avoid as much as possible false positive variants, we filtered out all genes with a Benjamini and Hochberg adjusted p-value above 0.05 . 
A

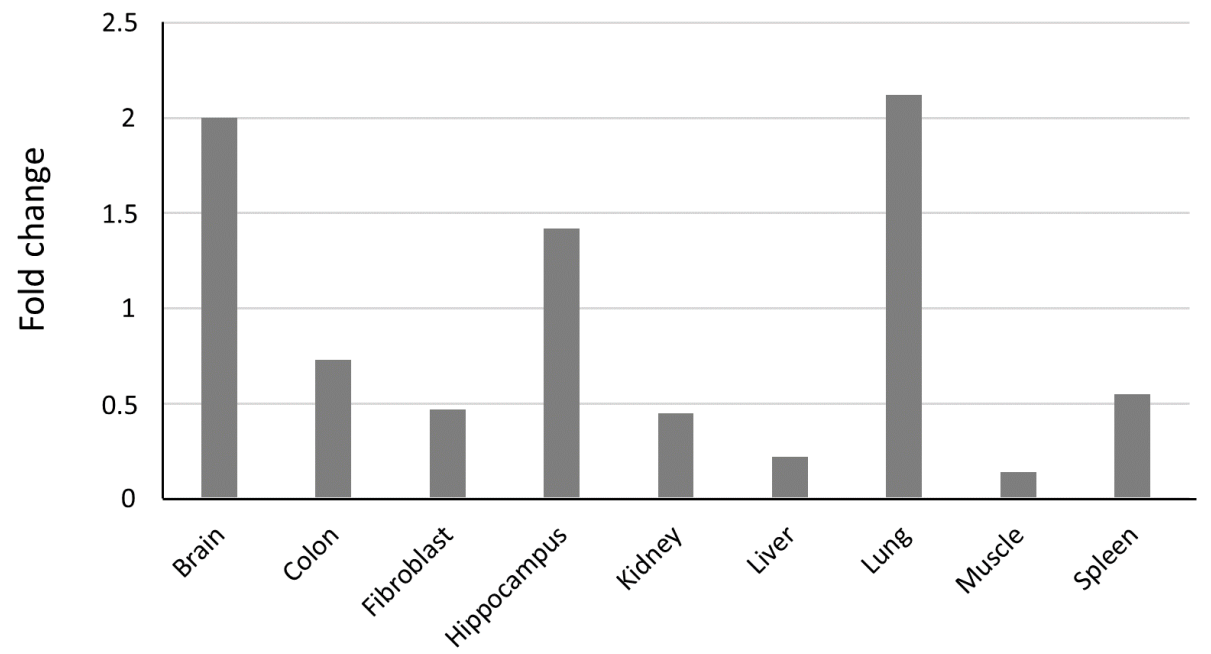

B

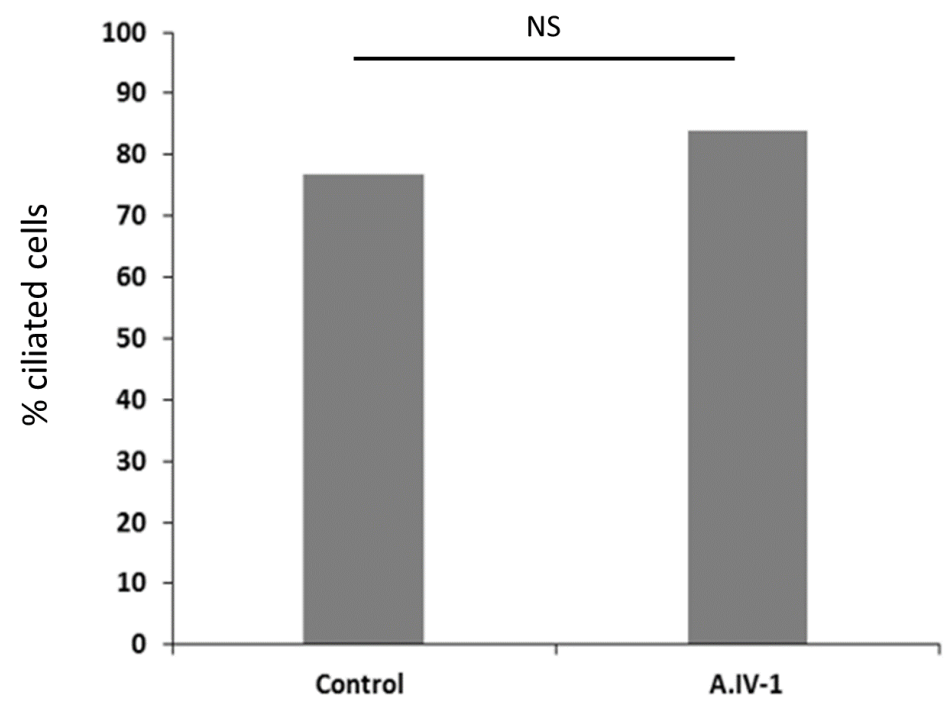

Supplementary Figure S1. A. Highest mRNA expression of IQCE in adult human brain/hippocampus and lung. B. Count of ciliated fibroblasts between A.IV-1 and control ( 100 cells per condition). Percentages are shown in histogram, N.S.: non-significant, Fisher exact test $0.224, P<0.05)$. 


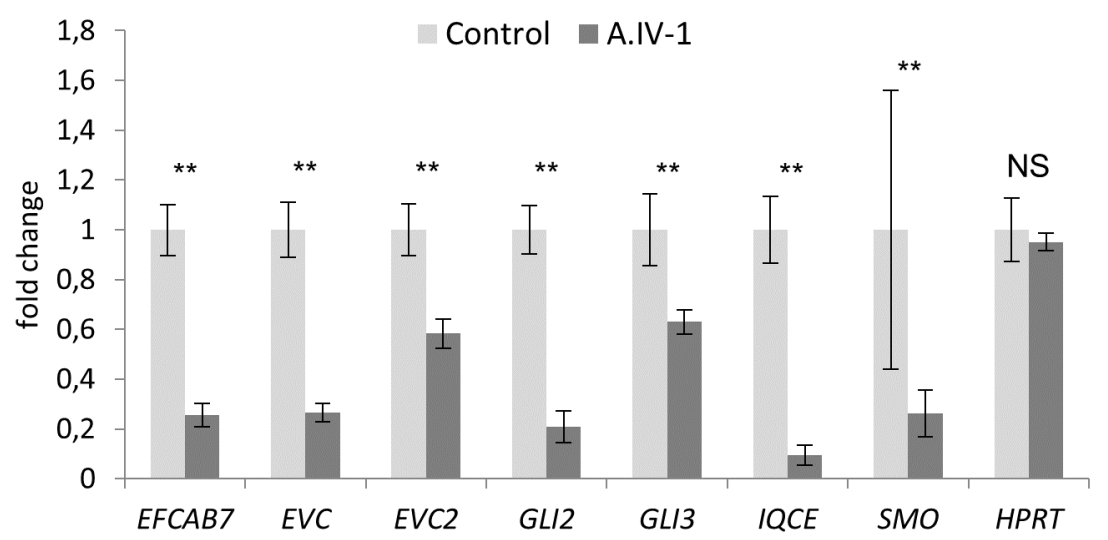

\section{Supplementary Figure S2. Expression levels of EVC-zone and GLI genes upon} ciliogenesis and Hh activation. Patient's (A.IV-1) and control cells upon treatment (-FCS + SAG $100 n M)$ were compared ( $n=4$ controls, $n=6$ patient). Statistical significance has been performed using the non-parametric Mann-Whitney test, $p=0.0095\left(^{* *}\right)$, NS : non-significant. 
A

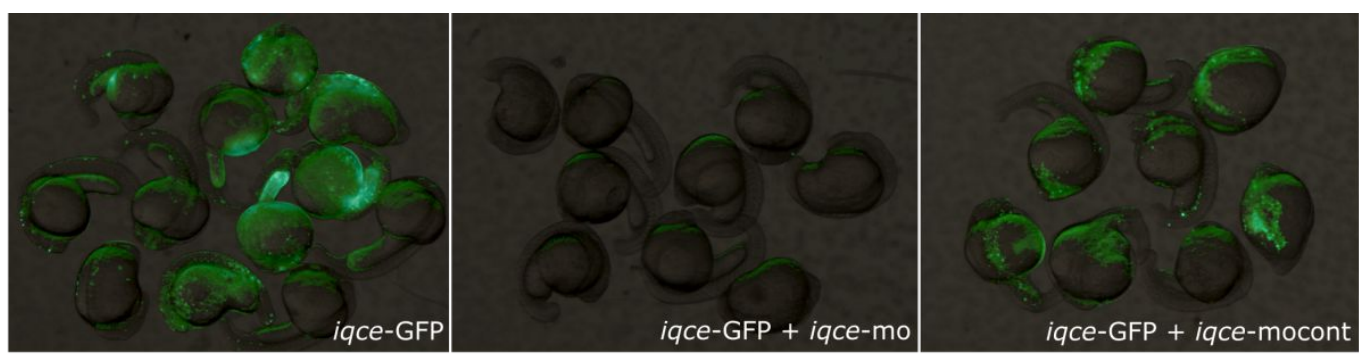

B
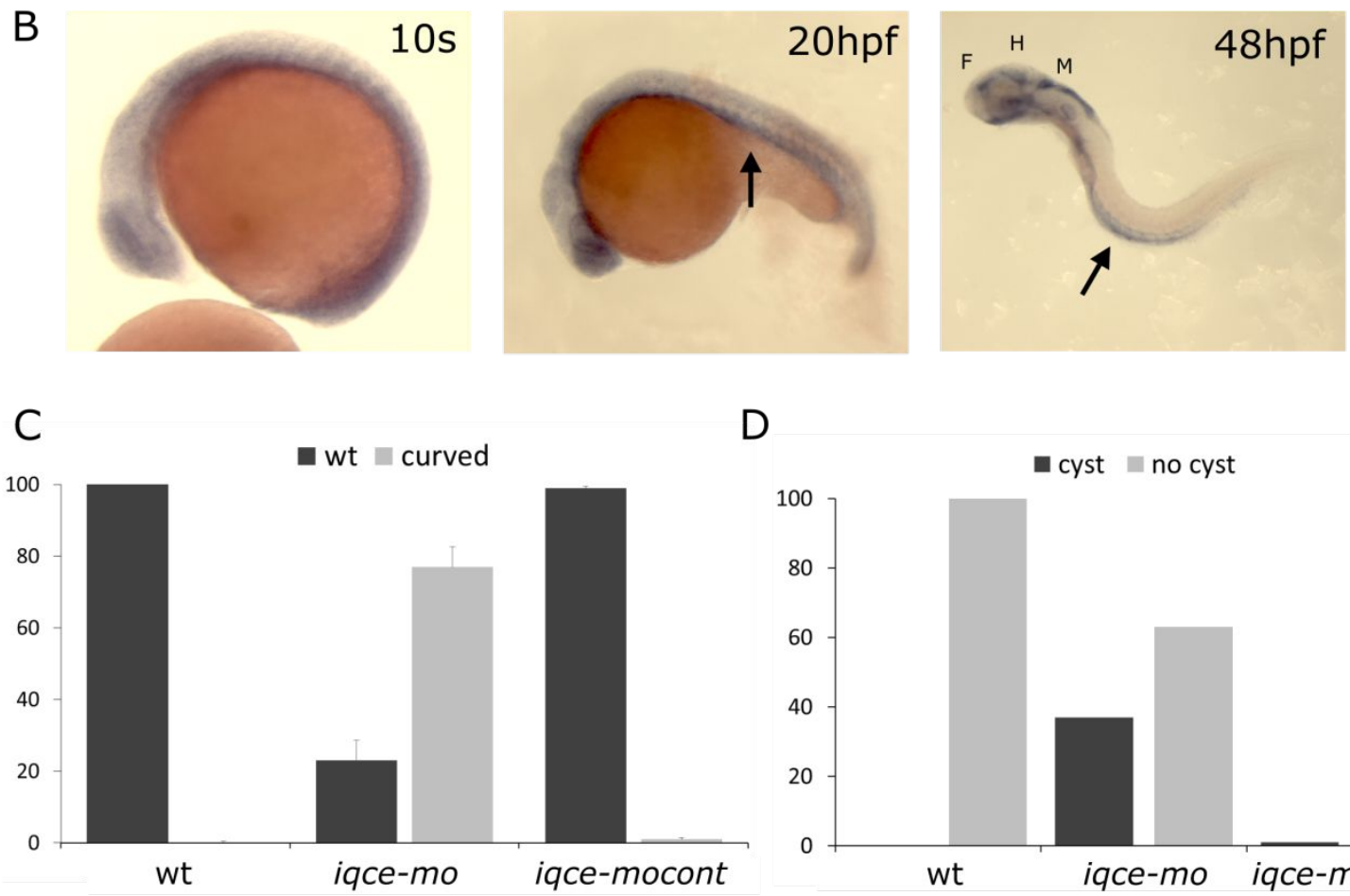

D

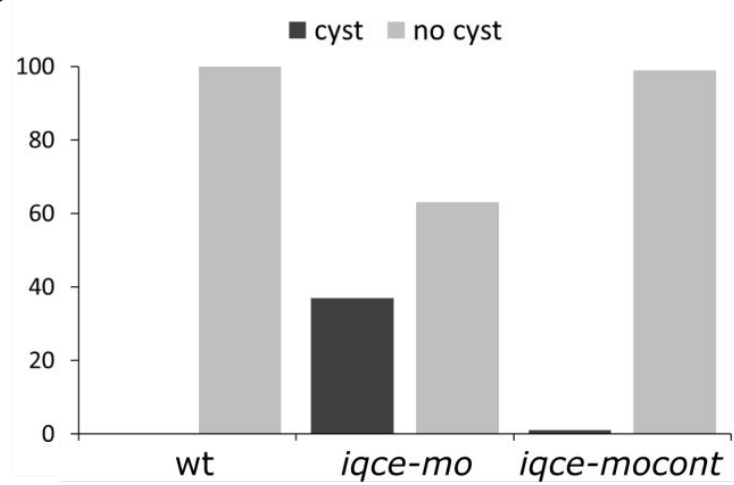

$\mathrm{E}$

$\mathrm{F}$
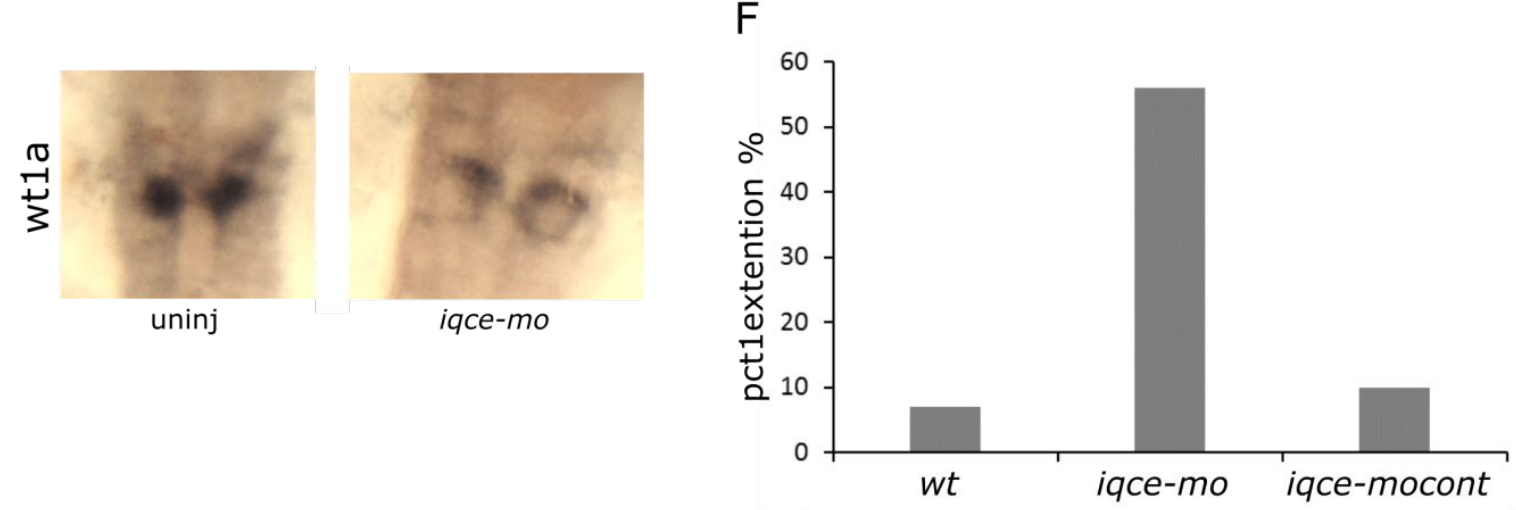

Supplementary Figure S3. Efficacy of iqce morpholinos. A. Morpholinos directed against the start codon of iqce blocks the expression of a construct containing iqce cDNA in frame with gfp (central panel). In contrast, 5 mismatch morpholinos did not reduce expression of the chimeric protein (left panel) compared to iqce-gfp alone (right panel). $24 \mathrm{hpf}$. B. in situ hybridization showing iqce expression pattern in 10 somites, 20 and 48 hpf embryos. Arrow indicates the pronephric duct. $\mathrm{F}$ : forebrain ventricule; $\mathrm{H}$ : hindbrain ventricule; $\mathrm{M}$ : midbrain ventricule. C. Chart showing the percentage of curved embryos in uninjected $(n=200)$, iqce- 
mo $(\mathrm{n}=120)$; and iqce-mocont $(\mathrm{n}=221)$ injected embryos. $48 \mathrm{hpf}$, data includes $\pm \mathrm{SD}$ and statistical significance was determined using the Student's t-test, all comparisons were significant with a p-value<0.05. D. Chart showing the percentage of cysts in uninjected $(\mathrm{n}=101)$, iqce-mo $(\mathrm{n}=70)$ and iqce-mocont $(\mathrm{n}=90)$ injected embryos. Statistical significance was determined using the Student's t-test, all comparisons were significant with a p-value<0.05. E. wt1a in situ hybridization showing expression within glomeruli in non-injected embryos and at the periphery of the cyst in morphants (48 hpf). F. Chart showing the percentage of embryos with ptc1 extension in 10 somites embryos $(n=191)$. 
Supplementary Table S1. Summary of the whole exome sequencing results. SNV: Single nucleotide variation, indel: gain or loss of up to 50 nucleotides at a single locus, SV: Structural Variation. Annotations are gathered using Alamut Batch v1.11, especially for the variation databases including gnomAD (v2.0.2, Oct. 2017), 1000 Genomes Project phase3 release (version 20150813 v5b) and the following predictions including phastCons (UCSC, 44 vertebrates). Effect on the splice has been evaluated using the MaxEntScan (Yeo \& Burge, 2004), NNSPLICE 0.9 (Reese, Eeckman, Kulp, \& Haussler, 1997) and Splice Site Finder (Shapiro \& Senapathy, 1987) by calculating score change between the wild type and the mutated sequences expressed as a percent differences. Missenses have been evaluated using default parameters from PolyPhen-2 (2.2.2) (Adzhubei et al., 2010) and SIFT 4.0.3 (Kumar, Henikoff, \& Ng, 2009). Default cut-offs used have been described in VaRank (Geoffroy et al., 2015) for both type of predictions. Exclusion of SV with a DGV (Gold standard from 20160515) frequency $>1 \%$ is done only with studies of more than 1000 individuals.

\begin{tabular}{|c|c|c|c|c|c|c|c|c|c|c|c|c|}
\hline & \multicolumn{3}{|c|}{ A.IV-1 } & \multicolumn{3}{|c|}{ B.II-2 } & \multicolumn{3}{|c|}{ B.II-3 } & \multicolumn{3}{|c|}{ C.IV-4 } \\
\hline & SNV & indel & sv & SNV & indel & sv & SNV & Indel & sv & SNV & Indel & sv \\
\hline Total number of variants & 83129 & 11664 & 31 & 81972 & 11319 & 17 & 82116 & 11195 & 16 & 78906 & 11727 & 31 \\
\hline $\begin{array}{l}\text { After exclusion of variants with an allele frequency }>1 \% \\
\text { (gnomAD, 1000G, internal exome database, DGV) }\end{array}$ & 9771 & 1125 & 16 & 7069 & 993 & 6 & 6856 & 943 & 9 & 5323 & 982 & 17 \\
\hline $\begin{array}{l}\text { After exclusion of SNV/indel in 5'UTR, 3'UTR, downstream, } \\
\text { upstream, intron and synonymous locations without local splice } \\
\text { effect prediction }\end{array}$ & 788 & 76 & I & 553 & 59 & l & 560 & 65 & 1 & 753 & 113 & 1 \\
\hline $\begin{array}{l}\text { After exclusion of missense with no deleterious effect } \\
\text { according to SIFT, PPH2 or PhastCons }\end{array}$ & 669 & 76 & 1 & 458 & 59 & I & 465 & 65 & 1 & 641 & 113 & l \\
\hline $\begin{array}{l}\text { After exclusion of variants not in a ciliary genes list (Nevers et } \\
\text { al., 2017) and not in non-syndromic polydactyly known genes }\end{array}$ & 52 & 6 & 1 & 31 & 2 & 2 & 40 & 4 & 2 & 78 & 15 & 1 \\
\hline $\begin{array}{l}\text { After selection of variants consistent with recessive } \\
\text { transmission (compound heterozygous, homozygous variants) }\end{array}$ & $\begin{array}{r}7 \text { hom. } \\
\text { SHA } \\
3 \text { comp. h }\end{array}$ & $\begin{array}{l}\text { IAAF1, IC } \\
\text { ? TULP1 } \\
\text { ARMC3, }\end{array}$ & $\begin{array}{l}1 A D D \\
111, M G A\end{array}$ & & $\begin{array}{l}0 \mathrm{hom} \\
1 \mathrm{com}\end{array}$ & $\begin{array}{l}\text { ozyg } \\
\text { ip. he }\end{array}$ & $\begin{array}{l}\text { ous vari } \\
\text { et. In } I Q C\end{array}$ & & & $\begin{array}{r}3 \text { hom.: } 2 \\
11 \mathrm{com} \\
D N A H 7 \\
\text { LRRCC1, } \\
\text { SMA }\end{array}$ & $\begin{array}{l}\text { T1, AGTF } \\
\text { et: CTDS } \\
\text { 'NLL1, El } \\
\text { C46, MR } \\
\text { 5, TMEN }\end{array}$ & $\begin{array}{l}\text { KDM4C } \\
\text { DDX1, } \\
\text { IFT20, } \\
\text {, PRKDC, } \\
\text { TPTE }\end{array}$ \\
\hline
\end{tabular}


Supplementary Table S2. Homozygous regions identified in individual A.IV-1.

\begin{tabular}{|c|c|c|c|c|c|c|c|c|}
\hline Chr & SNP1 & SNP2 & POS1 & POS2 & MB & \#SNP & DENSITY & \\
\hline 1 & rs4147830 & rs11431894 & 94544276 & 100680594 & 6.14 & 33 & 185.949 & \\
\hline 1 & rs4915053 & rs3761934 & 108113526 & 114189067 & 6.08 & 136 & 44.673 & \\
\hline 1 & 1_148741720_T_C & rs11428742 & 148741720 & 150550020 & 1.81 & 22 & 82.196 & \\
\hline 1 & rs 4636 & rs10927011 & 234744413 & 243579112 & 8.83 & 125 & 70.678 & \\
\hline 2 & rs7577088 & rs3764906 & 39233500 & 42588195 & 3.35 & 20 & 167.735 & \\
\hline 2 & rs522893 & 2_188250301_T_A & 169690729 & 188250301 & 18.56 & 224 & 82.855 & \\
\hline 2 & rs2304704 & rs12477076 & 190430177 & 191848312 & 1.42 & 23 & 61.658 & \\
\hline 3 & rs901854 & rs113574694 & 4856234 & 10089550 & 5.23 & 58 & 90.230 & \\
\hline 3 & rs9814557 & rs2246945 & 135720540 & 137843476 & 2.12 & 21 & 101.092 & \\
\hline 4 & rs13146318 & rs1051447 & 41615690 & 49063872 & 7.45 & 68 & 109.532 & \\
\hline 4 & rs4864727 & rs12621 & 54011424 & 57897570 & 3.89 & 61 & 63.707 & \\
\hline 4 & rs10434219 & rs344122 & 62598689 & 77637502 & 15.04 & 175 & 85.936 & \\
\hline 6 & rs2076506 & 6_32485853_C_T & 22570064 & 32485853 & 9.92 & 646 & 15.350 & \\
\hline 6 & rs1059547 & rs12189627 & 32557529 & 41059458 & 8.50 & 309 & 27.514 & TULP1 \\
\hline 7 & rs4916935 & rs798479 & 197267 & 2691201 & 2.49 & 41 & 60.828 & $I Q C E$ \\
\hline 7 & rs7794563 & rs80024169 & 5347295 & 14775824 & 9.43 & 78 & 120.879 & \\
\hline 7 & rs4718101 & rs6460315 & 64152353 & 66289040 & 2.14 & 38 & 56.229 & \\
\hline 8 & rs3989699 & rs13271468 & 7218621 & 12236202 & 5.02 & 132 & 38.012 & \\
\hline 8 & 8_12273335_G_A & rs66480340 & 12273335 & 24211885 & 11.94 & 202 & 59.102 & \\
\hline 8 & rs10086200 & rs59331088 & 61193588 & 74005131 & 12.81 & 72 & 177.938 & \\
\hline 8 & rs11777189 & rs12545587 & 99057150 & 136560952 & 37.50 & 204 & 183.842 & \\
\hline 9 & rs4977555 & rs11515 & 19550308 & 21968199 & 2.42 & 30 & 80.596 & \\
\hline 9 & rs7863560 & rs3217100 & 119495697 & 123734288 & 4.24 & 30 & 141.286 & \\
\hline 11 & 11_32452000_GG_- & rs2076623 & 32452000 & 33564123 & 1.11 & 25 & 44.485 & \\
\hline 11 & rs12274095 & rs7101792 & 69063767 & 71249386 & 2.19 & 28 & 78.058 & \\
\hline 11 & rs3740912 & rs4937391 & 125891269 & 128786294 & 2.90 & 32 & 90.470 & \\
\hline 12 & rs11054683 & rs1600 & 12247616 & 14656768 & 2.41 & 31 & 77.715 & \\
\hline 12 & rs3088008 & rs3210837 & 49724955 & 51685831 & 1.96 & 55 & 35.652 & \\
\hline 12 & rs74651927 & rs2242497 & 52695754 & 56992630 & 4.30 & 185 & 23.226 & \\
\hline 12 & rs1663564 & rs7488309 & 105546172 & 118520170 & 12.97 & 152 & 85.355 & \\
\hline 12 & rs1794956 & rs11059841 & 124848137 & 129190021 & 4.34 & 37 & 117.348 & \\
\hline 14 & rs34800262 & rs2249922 & 51370852 & 52906081 & 1.54 & 34 & 45.154 & \\
\hline 14 & rs2297113 & rs863091 & 58036494 & 59112475 & 1.08 & 23 & 46.782 & \\
\hline 14 & rs35533709 & rs2057482 & 60433392 & 62213848 & 1.78 & 30 & 59.349 & \\
\hline 15 & rs4923929 & rs555001 & 42439376 & 43545728 & 1.11 & 37 & 29.901 & \\
\hline 15 & rs11351249 & rs2623989 & 72105929 & 73545732 & 1.44 & 23 & 62.600 & \\
\hline 15 & rs752270 & rs4777755 & 87099537 & 93510603 & 6.41 & 171 & 37.492 & \\
\hline 16 & rs6564764 & rs931713 & 80581631 & 85023855 & 4.44 & 121 & 36.713 & \\
\hline 17 & rs8071836 & rs67749219 & 47238028 & 49711042 & 2.47 & 40 & 61.825 & \\
\hline
\end{tabular}




\section{Supplementary Table S3. List and characteristics of primers used in this study}

\begin{tabular}{|c|c|c|c|c|c|}
\hline Application & Gene & Exon & Forward (5'-3') & Reverse (5'-3') & $\begin{array}{l}\text { Size } \\
\text { (bp) }\end{array}$ \\
\hline \multirow[t]{4}{*}{ DNA } & IQCE & 12 & СTCTGAGCTCACAACCAACAG & GTTGCCCAAGGGTTCACA & 399 \\
\hline & & 16 & TGGTTTCTGGTGTTGTCAGG & TTTTCAGTGGCTGGTCAGAA & 382 \\
\hline & ATP6V1B1 & 3 & AGCCGTGGGAAGTAGGTGTT & ATGTCCCATTGACCCCACT & 385 \\
\hline & TULP1 & $12 / 13$ & GGGATGTAGGATCCCCTCA & CATGCCAGGAATGATGACG & 481 \\
\hline \multirow[t]{17}{*}{ cDNA } & $I Q C E$ & $3-4 / 5$ & ACATCGCCAAAGTCACCTTATC & AGGGCCTGGGTCAGACTT & 158 \\
\hline & GSTT1 & $2-3 / 3$ & ACCTTGACGGAGAGTGTGG & GCAGCTTCTCCGCAGAGT & 144 \\
\hline & SLC30A8 & $9-10 / 10$ & ССАTCTTACTCATGGAAGGTGTG & GCTGTAGCAACATGAGCTGAGA & 154 \\
\hline & RSPO1 & $4 / 5$ & TGGAGAGGAACGACATCCG & CCTTACACTTGGTGCAGAAGTTA & 156 \\
\hline & FGF9 & $2 / 3$ & GGCCTGGTCAGCATTCGAG & GTATCGCCTTCCAGTGTCCAC & 180 \\
\hline & SFRP1 & $1 / 2$ & GCTTCTACTGGCCCGAGATG & TGGCCTCAGATTTCAACTCGT & 180 \\
\hline & MECOM & $4 / 5$ & AAACTCGAAAGCGAGAATGATCT & TGGTGGCGAATTAAATTGGACTT & 185 \\
\hline & $A L P L$ & $6-7 / 7$ & AACATCAGGGACATTGACGTG & GTATCTCGGTTTGAAGCTCTTCC & 159 \\
\hline & IGF2 & $2-3 / 3-4$ & TTCTACTTCAGCAGGCCCG & GAAGTTGTCCGGAAGCACG & 168 \\
\hline & GAPDH & $4-5 / 6$ & GGAGCGAGATCCCTCCAAAAT & GGCTGTTGTCATACTTCTCATGG & 197 \\
\hline & HPRT1 & $1-2 / 2-3$ & CCTGGCGTCGTGATTAGTGAT & AGACGTTCAGTCCTGTCCATAA & 131 \\
\hline & $E F C A B 7$ & $6 / 6-7$ & GTCAGCAACCAGGAAGTTCA & CATGTGTTGCCAGTCCTTTATT & 115 \\
\hline & EVC2 & $6-7 / 8$ & CTCGTCACGGAACAGAACAC & CAGGTCAGCACAAGGGAGAG & 135 \\
\hline & $E V C$ & $2 / 4$ & TCGAAGGACAAGGAAGCTGT & TTTCATGCAGAGACGGGTTG & 148 \\
\hline & GLI2 & $2 / 3$ & AAGCAAGAAGCCAAAAGTGG & TGGTACCTTCCTTCCTGGTG & 188 \\
\hline & GL/3 & $2 / 3$ & GGCATTTTTTGGTCGAAGAGA & GGACATTCTGTGGCTGCATA & 238 \\
\hline & SMO & $2 / 3$ & TGCCCAAGTGTGAGAATGAC & TACCAGCTCTTGGGGTTGTC & 229 \\
\hline
\end{tabular}


Supplementary Table S4. Comparison of haplotypes of individuals A.IV-1 and B.II-2.

\begin{tabular}{|c|c|c|c|c|c|c|c|}
\hline ID & Gene & Chr & Position & Allele_1_A.IV-1 & Allele_2_A.IV-1 & $\begin{array}{l}\text { Allele_1_II- } \\
\text { 2_Family2 }\end{array}$ & $\begin{array}{l}\text { Allele_1_II- } \\
\text { 2_Family2 }\end{array}$ \\
\hline 7_2255974_C_T & MAD1L1 & 7 & 2255974 & $\mathrm{C}$ & C & C & $\mathrm{T}$ \\
\hline 7_2256017_C_A & MAD1L1 & 7 & 2256017 & $\mathrm{C}$ & C & C & $A$ \\
\hline 7_2257612_G_A & MAD1L1 & 7 & 2257612 & $A$ & $A$ & A & $G$ \\
\hline 7_2259134_C_T & MAD1L1 & 7 & 2259134 & C & C & C & $\mathrm{T}$ \\
\hline 7_2260715_G_T & MAD1L1 & 7 & 2260715 & $\mathrm{~T}$ & $\mathrm{~T}$ & $\mathrm{~T}$ & $\mathrm{~T}$ \\
\hline 7_2269552_G_T & MAD1L1 & 7 & 2269552 & $\mathrm{~T}$ & $\mathrm{~T}$ & $\mathrm{~T}$ & $G$ \\
\hline 7_2278663_T_A & FTSJ2 & 7 & 2278663 & $\mathrm{~T}$ & $\mathrm{~T}$ & A & A \\
\hline 7_2279482_A_C & FTSJ2 & 7 & 2279482 & $A$ & $A$ & $\mathrm{C}$ & C \\
\hline 7_2279851_T_C & FTSJ2 & 7 & 2279851 & $\mathrm{~T}$ & $\mathrm{~T}$ & C & C \\
\hline 7_2281754_C_T & FTSJ2 & 7 & 2281754 & C & C & C & $\mathrm{T}$ \\
\hline 7_2290328_C_T & SNX8 & 7 & 2290328 & C & C & C & $\mathrm{T}$ \\
\hline 7_2290336_T_G & $S N X 8$ & 7 & 2290336 & $\mathrm{~T}$ & $\mathrm{~T}$ & $\mathrm{~T}$ & G \\
\hline 7_2290372_C_T & $S N X 8$ & 7 & 2290372 & C & C & $\mathrm{T}$ & $\mathrm{T}$ \\
\hline 7_2290522_C_T & SNX8 & 7 & 2290522 & $\mathrm{~T}$ & $\mathrm{~T}$ & C & C \\
\hline 7_2292881_-_TG & $S N X 8$ & 7 & 2292881 & - & - & TG & TG \\
\hline 7_2296493_A_G & SNX8 & 7 & 2296493 & G & G & G & $G$ \\
\hline 7_2297006_A_G & SNX8 & 7 & 2297006 & A & A & G & G \\
\hline 7_2303000_G_A & $S N X 8$ & 7 & 2303000 & $A$ & $A$ & G & G \\
\hline 7_2303109_G_A & $S N X 8$ & 7 & 2303109 & G & G & G & $A$ \\
\hline 7_2303986_T_C & SNX8 & 7 & 2303986 & C & C & C & C \\
\hline 7_2304191_A_C & $S N X 8$ & 7 & 2304191 & A & A & C & C \\
\hline 7_2309159_-_A & SNX8 & 7 & 2309159 & - & - & - & A \\
\hline 7_2309174_G_A & SNX8 & 7 & 2309174 & G & G & G & A \\
\hline 7_2309185_G_A & SNX8 & 7 & 2309185 & G & G & G & $A$ \\
\hline 7_2309209_C_T & SNX8 & 7 & 2309209 & C & C & C & $\mathrm{T}$ \\
\hline 7_2314652_G_A & $S N X 8$ & 7 & 2314652 & G & G & G & A \\
\hline 7_2315008_G_C & SNX8 & 7 & 2315008 & G & G & C & C \\
\hline 7_2318254_ACTC_- & SNX8 & 7 & 2318254 & - & - & АСTC & АСTC \\
\hline 7_2318268_C_T & SNX8 & 7 & 2318268 & $\mathrm{~T}$ & $\mathrm{~T}$ & C & $\mathrm{C}$ \\
\hline 7_2318463_C_T & SNX8 & 7 & 2318463 & C & $\mathrm{T}$ & C & C \\
\hline 7_2318472_C_A & SNX8 & 7 & 2318472 & C & $A$ & C & C \\
\hline 7_2349470_T_C & $S N X 8$ & 7 & 2349470 & $\mathrm{~T}$ & $\mathrm{~T}$ & $\mathrm{~T}$ & C \\
\hline 7_2394991_C_T & EIF3B & 7 & 2394991 & $\mathrm{C}$ & C & C & $\mathrm{T}$ \\
\hline 7_2400306_T_C & EIF3B & 7 & 2400306 & C & C & C & $\mathrm{T}$ \\
\hline 7_2405885_C_A & EIF3B & 7 & 2405885 & A & A & A & C \\
\hline 7_2409035_C_T & EIF3B & 7 & 2409035 & $\mathrm{C}$ & C & C & $\mathrm{T}$ \\
\hline 7_2414142_A_G & EIF3B & 7 & 2414142 & G & G & G & A \\
\hline 7_2414365_A_G & EIF3B & 7 & 2414365 & A & A & A & G \\
\hline 7_2416822_A_G & EIF3B & 7 & 2416822 & $A$ & A & A & G \\
\hline 7_2418645_A_G & EIF3B & 7 & 2418645 & A & A & A & G \\
\hline 7_2433729_C_A & NA & 7 & 2433729 & C & C & C & A \\
\hline 7_2434522_G_A & NA & 7 & 2434522 & G & G & $A$ & A \\
\hline 7_2434596_TT_- & NA & 7 & 2434596 & TT & TT & TT & - \\
\hline 7_2434597_T_- & NA & 7 & 2434597 & $\mathrm{~T}$ & $\mathrm{~T}$ & $\mathrm{~T}$ & - \\
\hline
\end{tabular}




\begin{tabular}{|c|c|c|c|c|c|c|c|}
\hline 7_2472429_C_A & CHST12 & 7 & 2472429 & $\mathrm{C}$ & $\mathrm{C}$ & $\mathrm{C}$ & $A$ \\
\hline 7_2472455_A_T & CHST12 & 7 & 2472455 & A & $A$ & $A$ & $\mathrm{~T}$ \\
\hline 7_2489311_C_G & NA & 7 & 2489311 & C & C & C & G \\
\hline $\begin{array}{l}\text { 7_2489336_GTGTGT } \\
\text { GCACCT_- }\end{array}$ & NA & 7 & 2489336 & GTGTGTGCACCT & GTGTGTGCACCT & GTGTGTGCACCT & - \\
\hline 7_2489382_A_G & NA & 7 & 2489382 & A & A & A & G \\
\hline 7_2515382_-_C & NA & 7 & 2515382 & - & - & C & $\mathrm{C}$ \\
\hline 7_2517473_C_T & NA & 7 & 2517473 & C & $\mathrm{C}$ & $\mathrm{C}$ & $\mathrm{T}$ \\
\hline 7_2552986_A_C & LFNG & 7 & 2552986 & A & $A$ & $A$ & $\mathrm{C}$ \\
\hline 7_2553030_T_C & LFNG & 7 & 2553030 & $\mathrm{~T}$ & $\mathrm{~T}$ & $\mathrm{~T}$ & $\mathrm{C}$ \\
\hline 7_2557266_T_C & LFNG & 7 & 2557266 & $\mathrm{~T}$ & $\mathrm{~T}$ & C & C \\
\hline 7_2557377_T_C & LFNG & 7 & 2557377 & $\mathrm{~T}$ & $\mathrm{~T}$ & $\mathrm{~T}$ & $\mathrm{C}$ \\
\hline 7_2565268_C_A & LFNG/MIR4648 & 7 & 2565268 & C & C & $A$ & A \\
\hline 7_2566433_G_A & LFNG/MIR4648 & 7 & 2566433 & G & G & G & $A$ \\
\hline 7_2577691_C_G & BRAT1 & 7 & 2577691 & C & C & C & $\mathrm{G}$ \\
\hline 7_2577781_T_C & BRAT1 & 7 & 2577781 & $\mathrm{~T}$ & $\mathrm{~T}$ & $\mathrm{~T}$ & C \\
\hline 7_2578237_T_C & BRAT1 & 7 & 2578237 & $\mathrm{~T}$ & $\mathrm{~T}$ & $\mathrm{~T}$ & C \\
\hline 7_2578238_C_T & BRAT1 & 7 & 2578238 & C & C & C & $\mathrm{T}$ \\
\hline 7_2578434_G_T & BRAT1 & 7 & 2578434 & G & G & $G$ & $\mathrm{~T}$ \\
\hline 7_2578455_G_A & BRAT1 & 7 & 2578455 & G & $\mathrm{G}$ & G & A \\
\hline 7_2580914_C_T & BRAT1 & 7 & 2580914 & $\mathrm{C}$ & $\mathrm{C}$ & $\mathrm{C}$ & $\mathrm{T}$ \\
\hline 7_2581994_T_C & BRAT1 & 7 & 2581994 & $\mathrm{~T}$ & $\mathrm{~T}$ & $\mathrm{~T}$ & $\mathrm{C}$ \\
\hline $\begin{array}{l}7{ }^{7} 2582154{ }^{-} \\
\text {CCCCAGCTCCCG } \\
\text { GGTGT }\end{array}$ & BRAT1 & 7 & 2582154 & $\begin{array}{l}\text { CCCCAGCCTCC } \\
\text { CGGGTGT }\end{array}$ & $\begin{array}{c}\text { CCCCAGCCTCC } \\
\text { CGGGTGT }\end{array}$ & - & - \\
\hline 7_2582233_A_G & BRAT1 & 7 & 2582233 & A & $A$ & $A$ & G \\
\hline 7_2582381_T_A & BRAT1 & 7 & 2582381 & $\mathrm{~T}$ & $\mathrm{~T}$ & $\mathrm{~T}$ & $A$ \\
\hline 7_2582584_G_A & BRAT1 & 7 & 2582584 & G & G & G & $A$ \\
\hline 7_2583165_G_A & BRAT1 & 7 & 2583165 & G & G & G & A \\
\hline 7_2583328_C_T & BRAT1 & 7 & 2583328 & $\mathrm{C}$ & $\mathrm{C}$ & $\mathrm{C}$ & $\mathrm{T}$ \\
\hline 7_2584523_T_C & BRAT1 & 7 & 2584523 & $\mathrm{~T}$ & $\mathrm{~T}$ & $\mathrm{~T}$ & $\mathrm{C}$ \\
\hline 7_2587122_T_C & BRAT1 & 7 & 2587122 & $\mathrm{~T}$ & $\mathrm{~T}$ & $\mathrm{~T}$ & $\mathrm{C}$ \\
\hline 7_2598757_T_C & IQCE & 7 & 2598757 & $\mathrm{~T}$ & $\mathrm{~T}$ & $\mathrm{~T}$ & C \\
\hline 7_2604768_GTGT_- & $I Q C E$ & 7 & 2604768 & GTGT & GTGT & GTGT & - \\
\hline 7_2604769_T_* & IQCE & 7 & 2604769 & $\mathrm{~T}$ & $\mathrm{~T}$ & $\mathrm{~T}$ & * \\
\hline 7_2604816_C_T & $I Q C E$ & 7 & 2604816 & C & C & $\mathrm{C}$ & $\mathrm{T}$ \\
\hline 7_2611878_T_C & IQCE & 7 & 2611878 & $\mathrm{~T}$ & $\mathrm{~T}$ & $\mathrm{~T}$ & $\mathrm{C}$ \\
\hline 7_2612225_G_A & IQCE & 7 & 2612225 & A & $A$ & G & $G$ \\
\hline 7_2613042_G_C & $I Q C E$ & 7 & 2613042 & G & G & G & C \\
\hline 7_2618070_-_A & $I Q C E$ & 7 & 2618070 & - & - & - & $A$ \\
\hline 7_2619341_GT_- & IQCE & 7 & 2619341 & GT & GT & GT & - \\
\hline 7_2619363_G_C & $I Q C E$ & 7 & 2619363 & G & G & G & $\mathrm{C}$ \\
\hline 7_2623351_G_A & $I Q C E$ & 7 & 2623351 & G & G & G & $A$ \\
\hline 7_2623801_G_T & $I Q C E$ & 7 & 2623801 & G & G & G & $\mathrm{T}$ \\
\hline $\begin{array}{l}\text { 7_2625907_TGTCCC } \\
\text { GGAG_- }\end{array}$ & $I Q C E$ & 7 & 2625907 & - & - & - & TGTCCCGGAG \\
\hline $\begin{array}{l}\text { 7_2626039_CAGGGA } \\
\text { ATGG_- }\end{array}$ & $I Q C E$ & 7 & 2626039 & CAGGGAATGG & CAGGGAATGG & CAGGGAATGG & - \\
\hline 7_2627310_T_A & IQCE & 7 & 2627310 & $\mathrm{~T}$ & $\mathrm{~T}$ & $\mathrm{~T}$ & $A$ \\
\hline 7_2627579_AG_- & $I Q C E$ & 7 & 2627579 & $A G$ & $A G$ & - & - \\
\hline 7_2632477_A_G & IQCE & 7 & 2632477 & $A$ & $A$ & $A$ & $\mathrm{G}$ \\
\hline
\end{tabular}




\begin{tabular}{|c|c|c|c|c|c|c|}
\hline & & & & & & \\
\hline 7_2634518_AGAG_- & $I Q C E$ & 7 & 2634518 & AGAG & AGAG & AGAG \\
\hline 7_2636689_C_T & $I Q C E$ & 7 & 2636689 & C & C & C \\
\hline 7_2638347_C_G & $I Q C E$ & 7 & 2638347 & $\mathrm{C}$ & C & C \\
\hline 7_2640985_G_C & $I Q C E$ & 7 & 2640985 & G & G & $G$ \\
\hline 7_2647048_C_G & $I Q C E$ & 7 & 2647048 & C & C & C \\
\hline 7_2691201_C_A & TTYHЗ & 7 & 2691201 & A & A & C \\
\hline 7_2699687_-_G & TTYHЗ & 7 & 2699687 & - & - & G \\
\hline 7_2701809_T_C & TTYHЗ & 7 & 2701809 & $\mathrm{~T}$ & $\mathrm{C}$ & $\mathrm{T}$ \\
\hline 7_2742097_T_C & $A M Z 1$ & 7 & 2742097 & $\mathrm{C}$ & $\mathrm{C}$ & $\mathrm{T}$ \\
\hline 7_2749565_G_A & $A M Z 1$ & 7 & 2749565 & G & G & G \\
\hline 7_2752152_G_A & $A M Z 1$ & 7 & 2752152 & G & G & G \\
\hline 7_2752487_G_A & $A M Z 1$ & 7 & 2752487 & $A$ & $A$ & G \\
\hline 7_2773035_-_AT & GNA12 & 7 & 2773035 & - & - & AT \\
\hline 7_2802173_T_C & GNA12 & 7 & 2802173 & $\mathrm{~T}$ & $\mathrm{~T}$ & $\mathrm{~T}$ \\
\hline 7_2802522_T_C & GNA12 & 7 & 2802522 & $\mathrm{~T}$ & $\mathrm{~T}$ & $\mathrm{~T}$ \\
\hline 7_2834869_C_T & GNA12 & 7 & 2834869 & C & C & $\mathrm{T}$ \\
\hline 7_2854010_G_A & GNA12 & 7 & 2854010 & G & G & A \\
\hline 7_2946461_T_C & $C A R D 11$ & 7 & 2946461 & $\mathrm{C}$ & $\mathrm{C}$ & C \\
\hline 7_2952912_G_A & CARD11 & 7 & 2952912 & G & G & G \\
\hline 7_2957005_T_C & $C A R D 11$ & 7 & 2957005 & $\mathrm{C}$ & $\mathrm{C}$ & C \\
\hline 7_2958298_C_T & CARD11 & 7 & 2958298 & $\mathrm{C}$ & $\mathrm{C}$ & $\mathrm{T}$ \\
\hline 7_2962241_G_A & $C A R D 11$ & 7 & 2962241 & $A$ & $A$ & G \\
\hline 7_2962753_G_A & CARD11 & 7 & 2962753 & $G$ & $\mathrm{G}$ & G \\
\hline 7_2966445_T_G & $C A R D 11$ & 7 & 2966445 & $\mathrm{~T}$ & $\mathrm{~T}$ & $\mathrm{~T}$ \\
\hline 7_2966466_GT_- & CARD11 & 7 & 2966466 & GT & GT & GT \\
\hline 7_2968195_G_A & $C A R D 11$ & 7 & 2968195 & G & G & G \\
\hline 7_2968361_G_A & CARD11 & 7 & 2968361 & $G$ & $G$ & $\mathrm{G}$ \\
\hline 7_2968486_G_C & CARD11 & 7 & 2968486 & G & G & G \\
\hline 7_2985364_AC_- & CARD11 & 7 & 2985364 & $A$ & C & - \\
\hline
\end{tabular}


Supplementary Table S5. Differentially expressed transcripts in A.IV-1 fibroblasts.

\begin{tabular}{|c|c|c|c|c|c|}
\hline Gene name & Ensembl gene id & Description & $\log 2(\mathrm{FC})$ & P-value & Adjusted $p$-value \\
\hline$A B C A 8$ & ENSG00000141338 & $\begin{array}{l}\text { ATP-binding cassette, sub-family A } \\
\text { (ABC1), member } 8\end{array}$ & -4.205829134 & $3.52 \mathrm{E}-05$ & 0.019362186 \\
\hline AC011294.3 & ENSG00000233539 & Uncharacterized protein & 3.130226421 & $2.55 \mathrm{E}-07$ & 0.000427239 \\
\hline$A C C S$ & ENSG00000110455 & $\begin{array}{l}\text { 1-aminocyclopropane-1-carboxylate } \\
\text { synthase homolog (Arabidopsis)(non- } \\
\text { functional) }\end{array}$ & -0.953037549 & $1.28 \mathrm{E}-07$ & 0.00025011 \\
\hline ADAMTSL4 & ENSG00000143382 & ADAMTS-like 4 & -3.153983754 & $5.00 \mathrm{E}-07$ & 0.000732904 \\
\hline$A L D H 1 A 1$ & ENSG00000165092 & $\begin{array}{l}\text { aldehyde dehydrogenase } 1 \text { family, } \\
\text { member } \mathrm{A} 1\end{array}$ & -6.206001924 & 1.99E-08 & 5.40E-05 \\
\hline$A L P L$ & ENSG00000162551 & \begin{tabular}{|l} 
alkaline phosphatase, \\
liver/bone/kidney
\end{tabular} & -3.039649649 & 0.000113858 & 0.038536558 \\
\hline APBA2 & ENSG00000034053 & $\begin{array}{l}\text { amyloid beta (A4) precursor protein- } \\
\text { binding, family A, member } 2\end{array}$ & -2.732442847 & $6.79 \mathrm{E}-05$ & 0.028790694 \\
\hline ARHGAP28 & ENSG00000088756 & Rho GTPase activating protein 28 & 2.800539497 & $1.58 \mathrm{E}-05$ & 0.010684692 \\
\hline C10orf54 & ENSG00000107738 & $\begin{array}{l}\text { chromosome } 10 \text { open reading frame } \\
54\end{array}$ & -1.64124697 & $9.82 \mathrm{E}-05$ & 0.035643913 \\
\hline $\begin{array}{l}\text { C1QTNF9B- } \\
\text { AS1 }\end{array}$ & ENSG00000205861 & C1QTNF9B antisense RNA 1 & -2.047191404 & 3.88E-05 & 0.019780947 \\
\hline$C 2 C D 4 A$ & ENSG00000198535 & $\begin{array}{l}\text { C2 calcium-dependent domain } \\
\text { containing } 4 \mathrm{~A}\end{array}$ & 5.96982663 & 8.95E-07 & 0.001085914 \\
\hline$C 4 A$ & ENSG00000244731 & $\begin{array}{l}\text { complement component 4A (Rodgers } \\
\text { blood group) }\end{array}$ & -6.329532092 & $5.21 \mathrm{E}-11$ & 3.66E-07 \\
\hline CCDC180 & ENSG00000197816 & coiled-coil domain containing 180 & 2.351169896 & 3.81E-05 & 0.019780947 \\
\hline$C D 200$ & ENSG00000091972 & CD200 molecule & -4.053753384 & 0.000135641 & 0.044622222 \\
\hline CHI3L1 & ENSG00000133048 & $\begin{array}{l}\text { chitinase 3-like } 1 \text { (cartilage } \\
\text { glycoprotein-39) }\end{array}$ & 4.852100597 & $6.43 \mathrm{E}-07$ & 0.000837885 \\
\hline CHN1 & ENSG00000128656 & chimerin 1 & -1.617295158 & $2.25 \mathrm{E}-09$ & $1.32 \mathrm{E}-05$ \\
\hline CHRDL2 & ENSG00000054938 & chordin-like 2 & -4.241249176 & 0.000101018 & 0.03591767 \\
\hline CNTN1 & ENSG00000018236 & contactin 1 & -4.135254047 & $8.76 \mathrm{E}-05$ & 0.032879001 \\
\hline COL11A1 & ENSG00000060718 & collagen, type XI, alpha 1 & -4.558619592 & 6.30E-05 & 0.027357715 \\
\hline CSF2RA & ENSG00000198223 & $\begin{array}{l}\text { colony stimulating factor } 2 \text { receptor, } \\
\text { alpha, low-affinity (granulocyte- } \\
\text { macrophage) }\end{array}$ & 3.072895701 & $6.90 \mathrm{E}-05$ & 0.028906369 \\
\hline CYP21A1P & ENSG00000204338 & $\begin{array}{l}\text { cytochrome P450, family } 21 \text {, subfamily } \\
\text { A, polypeptide } 1 \text { pseudogene }\end{array}$ & -4.61429374 & $6.95 \mathrm{E}-06$ & 0.005439088 \\
\hline$D U S P 2$ & ENSG00000158050 & dual specificity phosphatase 2 & 3.527490256 & $1.70 \mathrm{E}-05$ & 0.01128586 \\
\hline ECM1 & ENSG00000143369 & extracellular matrix protein 1 & -1.261675626 & 5.81E-07 & 0.000786019 \\
\hline$E M C N$ & ENSG00000164035 & endomucin & 3.361027658 & 4.95E-05 & 0.0229427 \\
\hline EYA4 & ENSG00000112319 & eyes absent homolog 4 (Drosophila) & -4.200503943 & $4.26 \mathrm{E}-05$ & 0.020550766 \\
\hline$F A M 21 B$ & ENSG00000152726 & $\begin{array}{l}\text { family with sequence similarity } 21, \\
\text { member B }\end{array}$ & -4.995162913 & 7.93E-08 & 0.000164108 \\
\hline FGF9 & ENSG00000102678 & fibroblast growth factor 9 & 4.555259473 & 5.95E-08 & 0.000133394 \\
\hline$F O S B$ & ENSG00000125740 & $\begin{array}{l}\text { FBJ murine osteosarcoma viral } \\
\text { oncogene homolog B }\end{array}$ & 3.628551087 & 8.80E-05 & 0.032879001 \\
\hline$F Y B$ & ENSG00000082074 & FYN binding protein & -4.90648728 & 3.03E-05 & 0.016914087 \\
\hline GRPR & ENSG00000126010 & gastrin-releasing peptide receptor & -4.422707449 & 0.000144048 & 0.046095412 \\
\hline GSTT1 & ENSG00000184674 & glutathione S-transferase theta 1 & -9.843482395 & $6.94 \mathrm{E}-60$ & $2.44 \mathrm{E}-55$ \\
\hline HIVEP3 & ENSG00000127124 & $\begin{array}{l}\text { human immunodeficiency virus type I } \\
\text { enhancer binding protein } 3\end{array}$ & -1.599082154 & 6.64E-05 & 0.028486515 \\
\hline HOXC10 & ENSG00000180818 & homeobox C10 & -4.942455716 & $2.36 \mathrm{E}-05$ & 0.014065434 \\
\hline HOXC-AS3 & ENSG00000251151 & HOXC cluster antisense RNA 3 & -5.14737488 & 6.01E-05 & 0.026775571 \\
\hline IGF2 & ENSG00000167244 & $\begin{array}{l}\text { insulin-like growth factor } 2 \\
\text { (somatomedin } \mathrm{A})\end{array}$ & -4.063105964 & 3.83E-05 & 0.019780947 \\
\hline IGFBP1 & ENSG00000146678 & $\begin{array}{l}\text { insulin-like growth factor binding } \\
\text { protein } 1\end{array}$ & 2.926653654 & $1.58 \mathrm{E}-05$ & 0.010684692 \\
\hline
\end{tabular}




\begin{tabular}{|c|c|c|c|c|c|}
\hline IGFBP3 & ENSG00000146674 & $\begin{array}{l}\text { insulin-like growth factor binding } \\
\text { protein } 3\end{array}$ & 1.932377253 & 2.71E-06 & 0.002574523 \\
\hline IL17RB & ENSG00000056736 & interleukin 17 receptor B & 3.133016005 & 8.91E-05 & 0.032879001 \\
\hline IL20RA & ENSG00000016402 & interleukin 20 receptor, alpha & 3.104556086 & 3.12E-06 & 0.002749475 \\
\hline IQCE & ENSG00000106012 & IQ motif containing $E$ & -2.198435274 & $1.75 \mathrm{E}-43$ & 3.08E-39 \\
\hline IRF6 & ENSG00000117595 & interferon regulatory factor 6 & -3.931349479 & 7.74E-05 & 0.030946745 \\
\hline$I R X 1$ & ENSG00000170549 & iroquois homeobox 1 & 1.496134127 & $2.70 \mathrm{E}-05$ & 0.015596484 \\
\hline$I R X 2$ & ENSG00000170561 & iroquois homeobox 2 & 1.161748374 & 4.51E-09 & 1.77E-05 \\
\hline JAZF1-AS1 & ENSG00000234336 & JAZF1 antisense RNA 1 & -2.132735824 & 0.000107563 & 0.037119743 \\
\hline$K L K B 1$ & ENSG00000164344 & kallikrein B, plasma (Fletcher factor) 1 & 2.478417669 & 8.84E-05 & 0.032879001 \\
\hline LAMC1 & ENSG00000135862 & laminin, gamma 1 (formerly LAMB2) & -1.201150815 & $2.84 \mathrm{E}-07$ & 0.000453983 \\
\hline$L H X 9$ & ENSG00000143355 & LIM homeobox 9 & 4.298162126 & $1.45 \mathrm{E}-06$ & 0.001650048 \\
\hline LINC00537 & ENSG00000232815 & $\begin{array}{l}\text { long intergenic non-protein coding } \\
\text { RNA } 537\end{array}$ & -3.494641854 & 3.83E-05 & 0.019780947 \\
\hline LINC00578 & ENSG00000228221 & $\begin{array}{l}\text { long intergenic non-protein coding } \\
\text { RNA } 578\end{array}$ & -4.541164267 & 7.46E-09 & 2.39E-05 \\
\hline LINC01048 & ENSG00000230390 & $\begin{array}{l}\text { long intergenic non-protein coding } \\
\text { RNA } 1048\end{array}$ & 2.214309895 & 3.99E-05 & 0.019780947 \\
\hline LINC01117 & ENSG00000224577 & $\begin{array}{l}\text { long intergenic non-protein coding } \\
\text { RNA } 1117\end{array}$ & -2.17227763 & 2.17E-05 & 0.01316806 \\
\hline$L R R C 16 A$ & ENSG00000079691 & leucine rich repeat containing $16 \mathrm{~A}$ & -1.784218256 & $2.43 \mathrm{E}-05$ & 0.014245821 \\
\hline MAP6 & ENSG00000171533 & microtubule-associated protein 6 & -1.79656788 & $1.30 \mathrm{E}-06$ & 0.001530605 \\
\hline MAP7D2 & ENSG00000184368 & MAP7 domain containing 2 & 2.359306267 & 2.66E-06 & 0.002574523 \\
\hline MBP & ENSG00000197971 & myelin basic protein & -3.402601353 & 7.14E-05 & 0.029220727 \\
\hline MCCC1 & ENSG00000078070 & $\begin{array}{l}\text { methylcrotonoyl-CoA carboxylase } 1 \\
\text { (alpha) }\end{array}$ & -0.554719912 & 0.000128238 & 0.042584679 \\
\hline MECOM & ENSG00000085276 & MDS1 and EVI1 complex locus & -4.511721791 & 3.14E-09 & $1.58 \mathrm{E}-05$ \\
\hline METTL9 & ENSG00000197006 & methyltransferase like 9 & 0.906519962 & $3.86 \mathrm{E}-08$ & $9.70 \mathrm{E}-05$ \\
\hline MFGE8 & ENSG00000140545 & milk fat globule-EGF factor 8 protein & -2.694104724 & 7.44E-07 & 0.000934855 \\
\hline MLLT3 & ENSG00000171843 & $\begin{array}{l}\text { myeloid/lymphoid or mixed-lineage } \\
\text { leukemia (trithorax homolog, } \\
\text { Drosophila); translocated to, } 3\end{array}$ & 0.969013225 & $2.10 \mathrm{E}-05$ & 0.01316806 \\
\hline MRVI1-AS1 & ENSG00000177112 & MRVI1 antisense RNA 1 & -3.884388307 & $1.04 \mathrm{E}-05$ & 0.007509783 \\
\hline MYO16 & ENSG00000041515 & myosin XVI & -1.962481605 & 0.000117031 & 0.039233207 \\
\hline NDP & ENSG00000124479 & Norrie disease (pseudoglioma) & 3.545635871 & $1.83 \mathrm{E}-05$ & 0.011727901 \\
\hline NR5A2 & ENSG00000116833 & $\begin{array}{l}\text { nuclear receptor subfamily } 5 \text {, group A, } \\
\text { member } 2\end{array}$ & -4.138425423 & 8.61E-06 & 0.006447287 \\
\hline NTRK3 & ENSG00000140538 & $\begin{array}{l}\text { neurotrophic tyrosine kinase, receptor, } \\
\text { type } 3\end{array}$ & 2.777060262 & 2.95E-05 & 0.016740854 \\
\hline$P K I A$ & ENSG00000171033 & $\begin{array}{l}\text { protein kinase (cAMP-dependent, } \\
\text { catalytic) inhibitor alpha }\end{array}$ & -2.779337899 & 4.26E-05 & 0.020550766 \\
\hline$P N L D C 1$ & ENSG00000146453 & $\begin{array}{l}\text { poly(A)-specific ribonuclease (PARN)- } \\
\text { like domain containing } 1\end{array}$ & 2.95490495 & 2.27E-07 & 0.000399745 \\
\hline PNMA6C & ENSG00000235961 & $\begin{array}{l}\text { paraneoplastic Ma antigen family } \\
\text { member } 6 \mathrm{C}\end{array}$ & -4.033899706 & 0.000151809 & 0.047958078 \\
\hline POMZP3 & ENSG00000146707 & POM121 and ZP3 fusion & -1.717492119 & $3.12 \mathrm{E}-06$ & 0.002749475 \\
\hline POSTN & ENSG00000133110 & periostin, osteoblast specific factor & 2.83484857 & 2.14E-05 & 0.01316806 \\
\hline PRKAR1B & ENSG00000188191 & $\begin{array}{l}\text { protein kinase, cAMP-dependent, } \\
\text { regulatory, type I, beta }\end{array}$ & -1.732349926 & $1.78 \mathrm{E}-05$ & 0.011579369 \\
\hline PRLR & ENSG00000113494 & prolactin receptor & 3.300099077 & 0.000140582 & 0.045398939 \\
\hline PTK2B & ENSG00000120899 & protein tyrosine kinase 2 beta & -1.47679232 & $6.10 \mathrm{E}-05$ & 0.026827045 \\
\hline$R G C C$ & ENSG00000102760 & regulator of cell cycle & -3.93550099 & 7.06E-05 & 0.029220727 \\
\hline RHBDD3 & ENSG00000100263 & rhomboid domain containing 3 & -0.996433377 & $1.05 \mathrm{E}-05$ & 0.007509783 \\
\hline RSPO1 & ENSG00000169218 & R-spondin 1 & -4.313022549 & $5.98 \mathrm{E}-05$ & 0.026775571 \\
\hline S1PR1 & ENSG00000170989 & sphingosine-1-phosphate receptor 1 & -3.093512898 & $5.52 \mathrm{E}-06$ & 0.00452094 \\
\hline
\end{tabular}




\begin{tabular}{|c|c|c|c|c|c|}
\hline SCN1A & ENSG00000144285 & $\begin{array}{l}\text { sodium channel, voltage-gated, type I, } \\
\text { alpha subunit }\end{array}$ & -4.906276861 & 3.80E-06 & 0.003184547 \\
\hline SCN9A & ENSG00000169432 & $\begin{array}{l}\text { sodium channel, voltage-gated, type } \\
\text { IX, alpha subunit }\end{array}$ & -3.142530704 & $1.83 \mathrm{E}-06$ & 0.001898572 \\
\hline SDK1 & ENSG00000146555 & sidekick cell adhesion molecule 1 & 3.188897352 & 4.55E-05 & 0.021372366 \\
\hline SFRP1 & ENSG00000104332 & secreted frizzled-related protein 1 & -4.10661345 & $5.21 \mathrm{E}-07$ & 0.000732904 \\
\hline SHE & ENSG00000169291 & Src homology 2 domain containing $\mathrm{E}$ & 3.455259022 & 1.63E-06 & 0.001733572 \\
\hline$S L A$ & ENSG00000155926 & Src-like-adaptor & -2.701260288 & 3.99E-05 & 0.019780947 \\
\hline SLC16A11 & ENSG00000174326 & solute carrier family 16 , member 11 & -2.677392243 & 4.37E-05 & 0.020765473 \\
\hline SLC30A8 & ENSG00000164756 & $\begin{array}{l}\text { solute carrier family } 30 \text { (zinc } \\
\text { transporter), member } 8\end{array}$ & 6.104148946 & $1.35 \mathrm{E}-07$ & 0.000250798 \\
\hline SLC35D3 & ENSG00000182747 & solute carrier family 35 , member D3 & 9.179494017 & $2.46 \mathrm{E}-31$ & 2.89E-27 \\
\hline$S L C 4 A 3$ & ENSG00000114923 & $\begin{array}{l}\text { solute carrier family } 4 \text { (anion } \\
\text { exchanger), member } 3\end{array}$ & -2.366325383 & $7.28 \mathrm{E}-05$ & 0.029469479 \\
\hline SMOC1 & ENSG00000198732 & $\begin{array}{l}\text { SPARC related modular calcium } \\
\text { binding } 1\end{array}$ & -5.03813069 & 0.000104067 & 0.036268803 \\
\hline SPON2 & ENSG00000159674 & spondin 2 , extracellular matrix protein & -2.639592845 & 0.000112611 & 0.038484373 \\
\hline$S R D 5 A 2$ & ENSG00000049319 & \begin{tabular}{|l|} 
steroid-5-alpha-reductase, alpha \\
polypeptide 2 (3-oxo-5 alpha-steroid \\
delta 4-dehydrogenase alpha 2) \\
\end{tabular} & 4.448074003 & 8.97E-05 & 0.032879001 \\
\hline STAG3L2 & ENSG00000160828 & stromal antigen 3-like 2 (pseudogene) & -0.881204831 & $9.94 \mathrm{E}-05$ & 0.035716372 \\
\hline TCF21 & ENSG00000118526 & transcription factor 21 & -6.04609974 & $1.52 \mathrm{E}-06$ & 0.001676294 \\
\hline ULK4 & ENSG00000168038 & unc-51 like kinase 4 & 1.147447551 & 6.46E-09 & $2.28 \mathrm{E}-05$ \\
\hline WASF3 & ENSG00000132970 & WAS protein family, member 3 & 0.972046987 & 2.81E-06 & 0.002603617 \\
\hline$X K R 9$ & ENSG00000221947 & $\begin{array}{l}\text { XK, Kell blood group complex subunit- } \\
\text { related family, member } 9\end{array}$ & -1.989973823 & 0.000159916 & 0.049814665 \\
\hline ZBTB16 & ENSG00000109906 & $\begin{array}{l}\text { zinc finger and BTB domain containing } \\
16\end{array}$ & -4.844890791 & 7.83E-05 & 0.030946745 \\
\hline ZFPM2 & ENSG00000169946 & $\begin{array}{l}\text { zinc finger protein, FOG family } \\
\text { member } 2\end{array}$ & -3.60011069 & $5.25 \mathrm{E}-14$ & $4.62 \mathrm{E}-10$ \\
\hline ZNF804A & ENSG00000170396 & zinc finger protein $804 \mathrm{~A}$ & -5.483825868 & 4.34E-09 & 1.77E-05 \\
\hline
\end{tabular}


Supplementary Table S6. GO enrichment analysis. Show the distribution of GO terms exhibiting statistical significant differences of mis-regulated transcripts in A.IV-1 fibroblast using WebGestalt 2019 (Wang, Vasaikar, Shi, Greer, \& Zhang, 2017).

\begin{tabular}{|c|c|c|c|c|c|c|c|c|c|}
\hline GO terms & Description & $\begin{array}{c}\text { Gene } \\
\text { set size }\end{array}$ & Overlap & Expected Value & Enrichment Ratio & pValue & FDR & overlap_ld & user_Id \\
\hline GO:0009888 & tissue development & 1613 & 24 & 7.625171279802685 & 3.1474702822178497 & $1.528766712111107 \mathrm{e}-7$ & 0012670418509976855 & 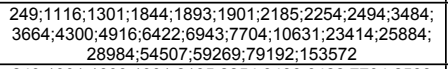 & 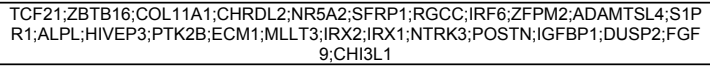 \\
\hline GO:0001503 & ossification & 339 & 11 & 1.6025623458481775 & 6.864007524261469 & $4.940246167706164 \mathrm{e}-7$ & 0020472380118974343 & $249 ; 1301 ; 1893 ; 1901 ; 2185 ; 2254 ; 33486 ; 6422 ; 7704 ; 2588$ & SMOC1;ZBTB16;COL11A1;CHRDL2;SFRP1;S1PR1;ALPL;PTK2B;ECM1;|GFBP3;FGF9 \\
\hline Go:0030038 & $\begin{array}{l}\text { Iractile actin filamen } \\
\text { assembly }\end{array}$ & 76 & 5 & 0.35927651411345574 & 13.916857360793287 & $2.9031950272262463 \mathrm{e}-5$ & 0.060154200964127824 & $1901 ; 2185 ; 6422 ; 28984 ; 55604$ & SFRP1;RGCC;S1PR1;CARMLL1;PTK2B \\
\hline G0:0043149 & stress fiber as & 76 & 5 & 0.35927651411345574 & 13.916857360793287 & $2.9031950272262463 \mathrm{e}-5$ & 0.060154200964127824 & $: 2185 ; 6422$ & SFRP1;RGCC;S1PR1;CARMIL1;PTK2B \\
\hline Go:0048584 & $\begin{array}{l}\text { positive regulation of response to } \\
\text { stimulus }\end{array}$ & 1768 & 21 & 8.35790627569197 & 2.512590989573087 & 4.138463705938289e-5 & 0.06101540527461857 & $\begin{array}{l}1116 ; 1123 ; 1893 ; 1901 ; 2070 ; 2185 ; 2254 ; 2533 ; 3486 ; 3381 \\
8 ; 4300 ; 4916 ; 5618 ; 642226653 ; 101417 ; 28984 ; 53832 ; 555 \\
40 ; 126669 ; 284654\end{array}$ & 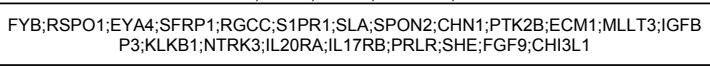 \\
\hline G0:0007379 & & $\frac{15}{160}$ & 3 & 0.07090 & 3768116 & $4.417138412737831 \mathrm{e}-5$ & 006101540527 & & \\
\hline G0:0030278 & & $\frac{169}{876}$ & $\begin{array}{ll}6 \\
13\end{array}$ & $\begin{array}{c}0.7989175116470265 \\
4.14113455741299\end{array}$ & $\begin{array}{l}7.510162078723952 \\
3.1392363179141025\end{array}$ & $\frac{1.4457514683041683 \mathrm{e}-4}{2.015277408380367 \mathrm{e}-4}$ & $\begin{array}{l}0.12546232977013955 \\
0.12546232977013955\end{array}$ & 6;;1301;1844; & 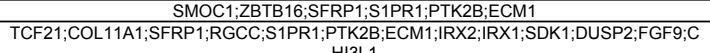 \\
\hline Go:0001501 & letal system development & 439 & 9 & 2.075294601260619 & 4.33673368327226 & $2.1232290176698143 \mathrm{e}-4$ & 0.12546232977013955 & $\begin{aligned} 84 ; 79192 ; 153532 ; 2221935 \\
249 ; 1116 ; 1301 ; 2254 ; 3226 ; 6422 ; 704 ; 10631 ; 25884 \\
\end{aligned}$ & $\begin{array}{c}\text { H13L1 } \\
\text { HOXC10;ZBTB16;COL11A1;CHRLL2;SFR1;ALPL;POSTN;FGF9;CHI3L1 }\end{array}$ \\
\hline GO:0072047 & $\begin{array}{l}\text { proximal/distal pattern formation } \\
\text { invulued in nephron development }\end{array}$ & 5 & 2 & 0.023636612770622088 & 84.61449275362318 & $2.182366244216638 \mathrm{e}-4$ & 0.12546232977013955 & $79192 ; 153572$ & 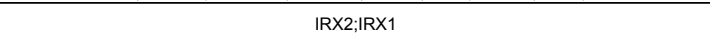 \\
\hline
\end{tabular}


Supplementary Table S7. Summary of transcriptome mapping results.

\begin{tabular}{|c|c|c|c|c|c|}
\hline Sample ID & \#total reads & \#aligned reads & $\begin{array}{c}\text { \% aligned } \\
\text { reads }\end{array}$ & $\begin{array}{c}\text { \#uniquely } \\
\text { mapped }\end{array}$ & $\begin{array}{c}\text { \% uniquely } \\
\text { aligned reads }\end{array}$ \\
\hline A.IV-1.1 & $306,265,974$ & $303,190,132$ & 99.00 & $293,189,972$ & 96.70 \\
\hline A.IV-1.2 & $344,782,588$ & $341,370,324$ & 99.01 & $330,182,914$ & 96.72 \\
\hline ARN1.1 & $338,129,498$ & $335,557,854$ & 99.24 & $325,618,112$ & 97.04 \\
\hline ARN1.2 & $301,339,526$ & $298,096,006$ & 98.92 & $289,521,446$ & 97.12 \\
\hline ARN2.1 & $311,917,536$ & $307,641,094$ & 98.63 & $296,837,798$ & 96.49 \\
\hline ARN2.2 & $336,751,354$ & $333,728,602$ & 99.10 & $322,903,810$ & 96.76 \\
\hline ARN3.1 & $403,718,870$ & $400,433,942$ & 99.19 & $383,740,470$ & 95.83 \\
\hline ARN3.2 & $299,879,030$ & $296,763,108$ & 98.96 & $286,203,188$ & 96.44 \\
\hline ARN4.1 & $318,588,494$ & $315,510,058$ & 99.03 & $304,438,002$ & 96.49 \\
\hline ARN4.2 & $320,107,070$ & $316,729,460$ & 98.94 & $304,559,776$ & 96.16 \\
\hline ARN5.1 & $363,584,984$ & $360,496,138$ & 99.15 & $349,081,194$ & 96.83 \\
\hline ARN6.1 & $324,984,406$ & $321,703,444$ & 98.99 & $311,631,678$ & 96.87 \\
\hline ARN6.2 & $364,763,088$ & $361,575,516$ & 99.13 & $349,853,468$ & 96.76 \\
\hline
\end{tabular}




\section{SUPPLEMENTARY REFERENCES}

Adzhubei, I. A., Schmidt, S., Peshkin, L., Ramensky, V. E., Gerasimova, A., Bork, P., . . Sunyaev, S. R. (2010). A method and server for predicting damaging missense mutations. Nature Methods, 7(4), 248249. doi:10.1038/nmeth0410-248

Geoffroy, V., Pizot, C., Redin, C., Piton, A., Vasli, N., Stoetzel, C., . . Muller, J. (2015). VaRank: a simple and powerful tool for ranking genetic variants. PeerJ, 3, e796. doi:10.7717/peerj.796

Kim, D., Pertea, G., Trapnell, C., Pimentel, H., Kelley, R., \& Salzberg, S. L. (2013). TopHat2: accurate alignment of transcriptomes in the presence of insertions, deletions and gene fusions. Genome Biol, 14(4), R36. doi:10.1186/gb-2013-14-4-r36

Kumar, P., Henikoff, S., \& Ng, P. C. (2009). Predicting the effects of coding non-synonymous variants on protein function using the SIFT algorithm. Nature protocols, 4(7), 1073-1081. doi:10.1038/nprot.2009.86

Langmead, B., Trapnell, C., Pop, M., \& Salzberg, S. L. (2009). Ultrafast and memory-efficient alignment of short DNA sequences to the human genome. Genome Biol, 10(3), R25. doi:10.1186/gb-2009-10-3r25

Love, M. I., Huber, W., \& Anders, S. (2014). Moderated estimation of fold change and dispersion for RNA-seq data with DESeq2. Genome Biol, 15(12), 550. doi:10.1186/s13059-014-0550-8

Nevers, Y., Prasad, M. K., Poidevin, L., Chennen, K., Allot, A., Kress, A., . . Lecompte, O. (2017). Insights into Ciliary Genes and Evolution from Multi-Level Phylogenetic Profiling. Mol Biol Evol, 34(8), 20162034. doi:10.1093/molbev/msx146

Reese, M. G., Eeckman, F. H., Kulp, D., \& Haussler, D. (1997). Improved splice site detection in Genie. J Comput Biol, 4(3), 311-323.

Shapiro, M. B., \& Senapathy, P. (1987). RNA splice junctions of different classes of eukaryotes: sequence statistics and functional implications in gene expression. Nucleic Acids Res, 15(17), 71557174.

Wang, J., Vasaikar, S., Shi, Z., Greer, M., \& Zhang, B. (2017). WebGestalt 2017: a more comprehensive, powerful, flexible and interactive gene set enrichment analysis toolkit. Nucleic Acids Research, 45(W1), W130-W137. doi:10.1093/nar/gkx356

Yeo, G., \& Burge, C. B. (2004). Maximum entropy modeling of short sequence motifs with applications to RNA splicing signals. J Comput Biol, 11(2-3), 377-394. doi:10.1089/1066527041410418 


\section{SUPPLEMENTARY INFORMATION}

\section{Sanger Sequencing}

Sanger sequencing was performed by way of PCR amplification with 50ng of genomic DNA template. The primers were designed with Primer 3 (http://frodo.wi.mit.edu/primer3). Bidirectional sequencing of the purified PCR products was performed by Eurofins Genomics Sequencing Facilities (https://www.eurofinsgenomics.eu/en/custom-dna-sequencing).

\section{Control screening}

IQCE exon 12 was tested in 93 control DNA of matched origin (Algerian) and 93 multi ethnic. High resolution melting (HRM) assay was performed in a $20 \mu$ reaction volume containing 50 ng of template DNA, $15 \mu$ Precision Melt Supermix (Bio-Rad) and $10 \mu \mathrm{mol}$ of each primer of the primer set. PCR was performed using a CFX96 (Bio-Rad) thermal cycler and consisted of predenaturation $\left(98^{\circ} \mathrm{C}\right.$ for $2 \mathrm{~min}$ ) followed by 35 cycles of amplification involving denaturation $\left(98^{\circ} \mathrm{C}\right.$ for $\left.5 \mathrm{~s}\right)$, annealing $\left(60^{\circ} \mathrm{C}\right.$ for $\left.10 \mathrm{~s}\right)$ and a melting curve involving denaturation $\left(95^{\circ} \mathrm{C}\right.$ for $30 \mathrm{~s})$, annealing $\left(70^{\circ} \mathrm{C}\right.$ for $\left.30 \mathrm{~s}\right)$ and a melt curve $\left(72-95^{\circ} \mathrm{C}\right.$ with an increment of $0.2^{\circ} \mathrm{C}$ per 10 s). Data generated were analyzed using Precision Melt Analysis software (Bio-Rad). In HRM analysis, differences in Tm and normalized curve shape were used together to discriminate even the most difficult-to-detect sequence variation.

\section{Bioinformatic pipeline RNA seq}

Image analysis and base calling were performed using RTA v.2.7.3 and blc2fastq v.2.17.1.14 (Illumina). Sequence reads were mapped onto hg19/GRCh37 assembly of human genome using Tophat 2.0.14 (Kim et al., 2013) and bowtie version 2-2.1.0 (Langmead, Trapnell, Pop, \& Salzberg, 2009), A summary of the mapping results is available in Supplementary Table 7. Gene expression was quantified from uniquely aligned reads using HTSeq-0.6.1 with gene annotations from Ensembl release 75 and intersection non-empty mode.

\section{Differential expression RNA-seq}

In order to identify variation in mRNA expression due to the absence of IQCE in the patient cells, comparisons of read counts were performed using $\mathrm{R} 3.2 .5$ with the statistical method proposed by Anders and Huber implemented in the DESeq2 v.1.10.1 Bioconductor package 
(Love, Huber, \& Anders, 2014). The generated data were explored and visualized to assess and check data quality and to eventually remove bad quality data. Especially, PCA was built after stabilizing variance using the regularized log transformation method or RSeqQC was used to do some quality controls such as gene body coverage. Only genes with | log2 foldchange $\mid>0.5$ were considered, as we expected to identify a large amount of change between transcriptomic results from one patient compared to other individuals (indirect consequences of the pathogenic mutation or unrelated events due to variability between humans). To avoid as much as possible false positive variants, we filtered out all genes with a Benjamini and Hochberg adjusted p-value above 0.05 . 
A

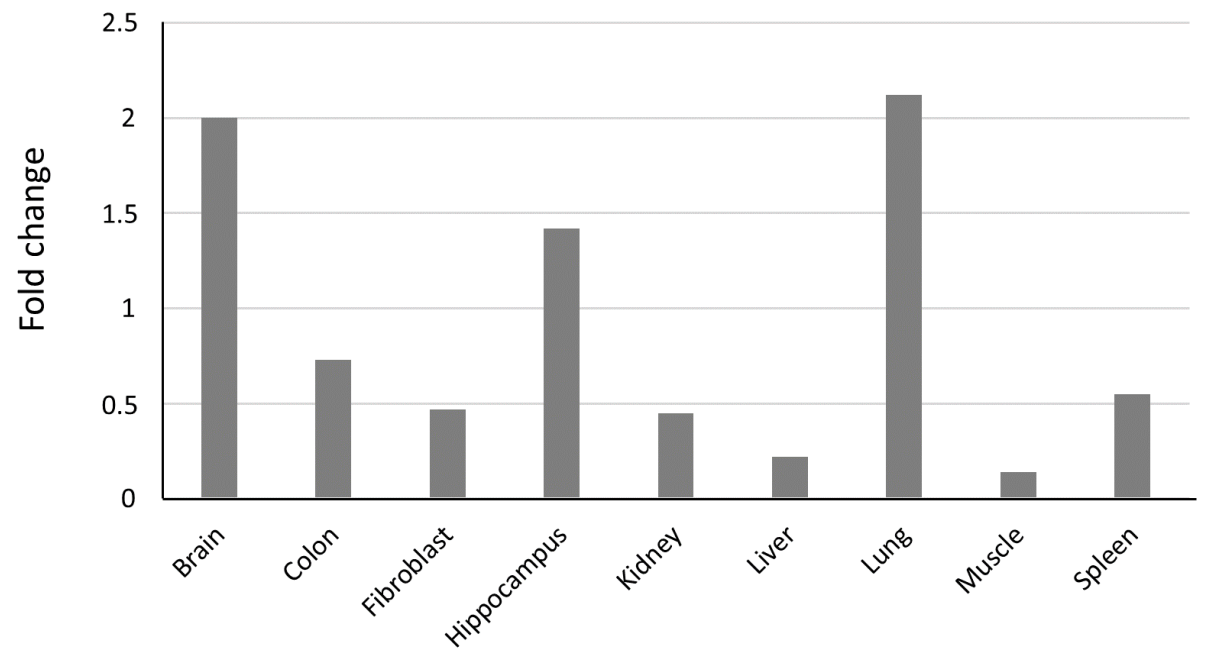

B

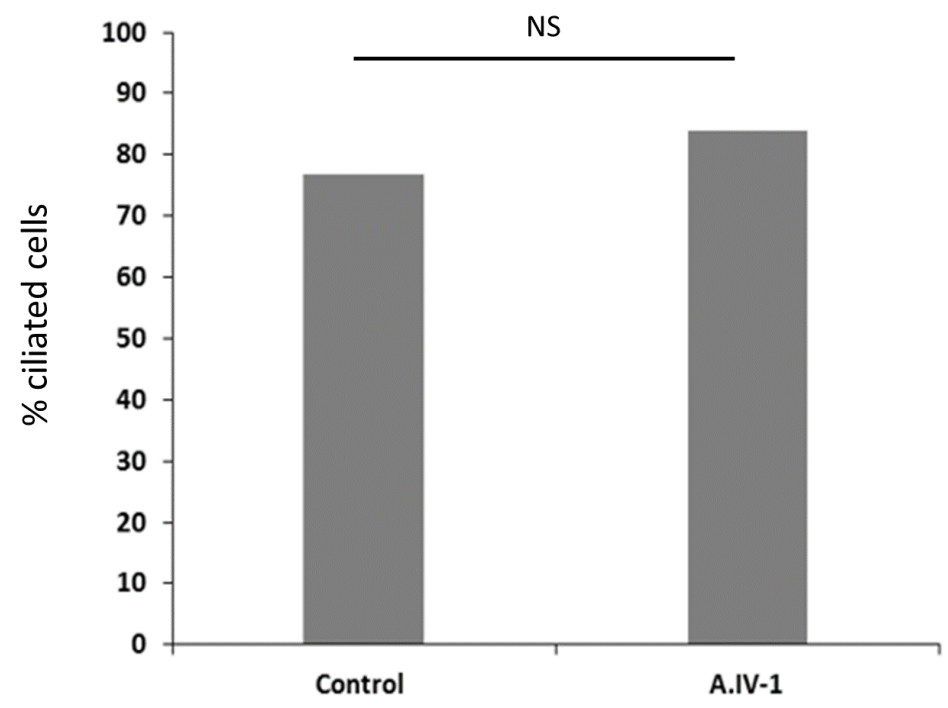

Supplementary Figure S1. A. Highest mRNA expression of IQCE in adult human brain/hippocampus and lung. B. Count of ciliated fibroblasts between A.IV-1 and control ( 100 cells per condition). Percentages are shown in histogram, N.S.: non-significant, Fisher exact test $0.224, P<0.05)$. 


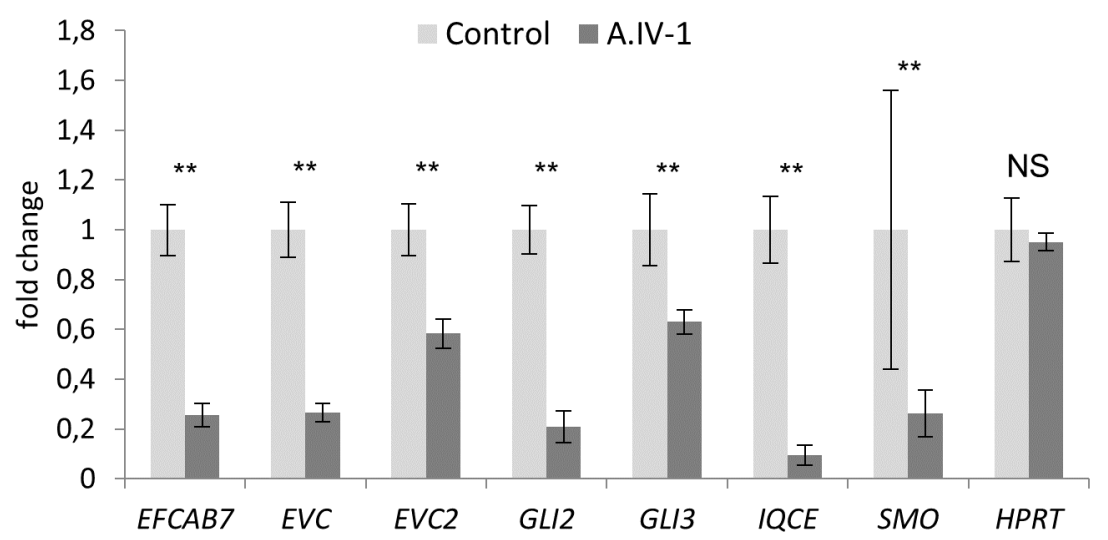

Supplementary Figure S2. Expression levels of EVC-zone and GLI genes upon ciliogenesis and Hh activation. Patient's (A.IV-1) and control cells upon treatment (-FCS + SAG $100 n M$ ) were compared ( $n=4$ controls, $n=6$ patient). Statistical significance has been performed using the non-parametric Mann-Whitney test, $p=0.0095\left(^{* *}\right)$, NS : non-significant. 
A

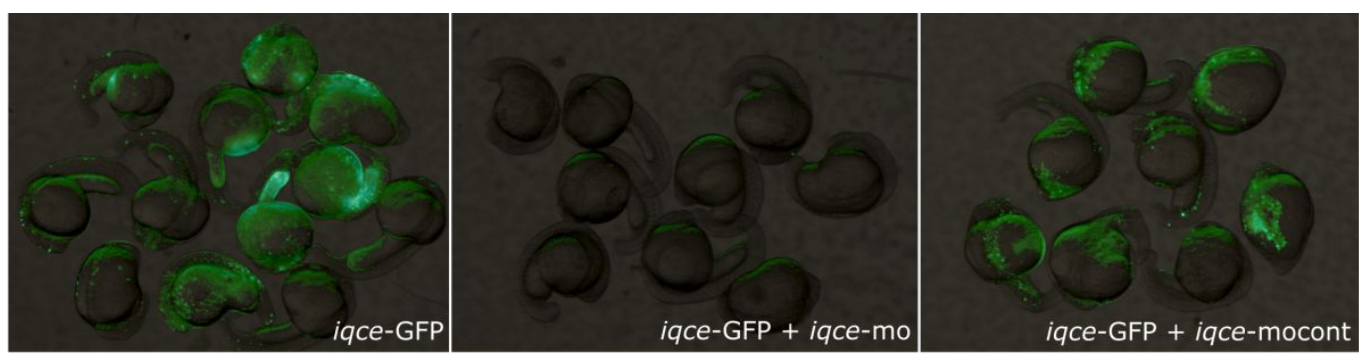

B
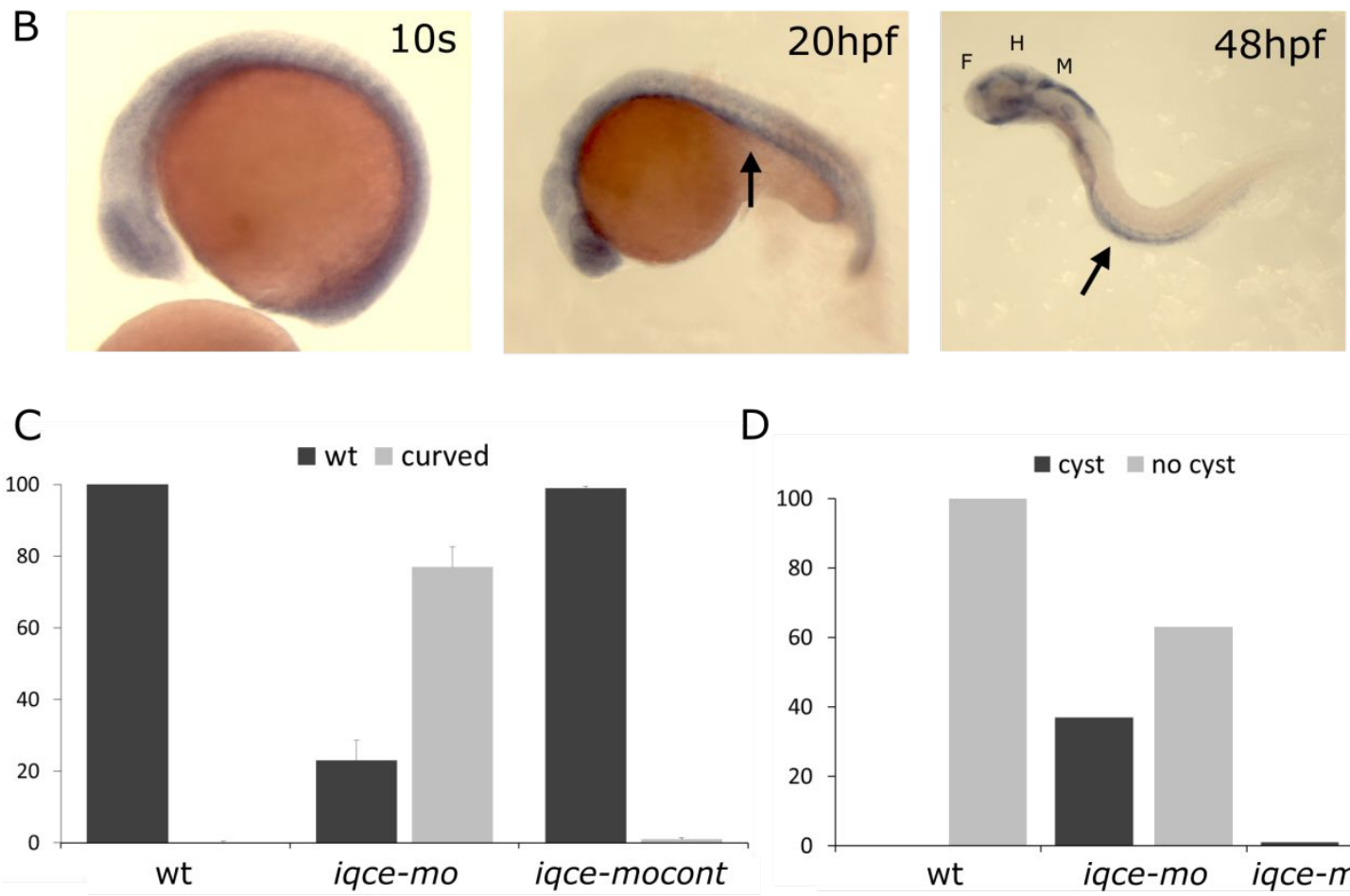

D

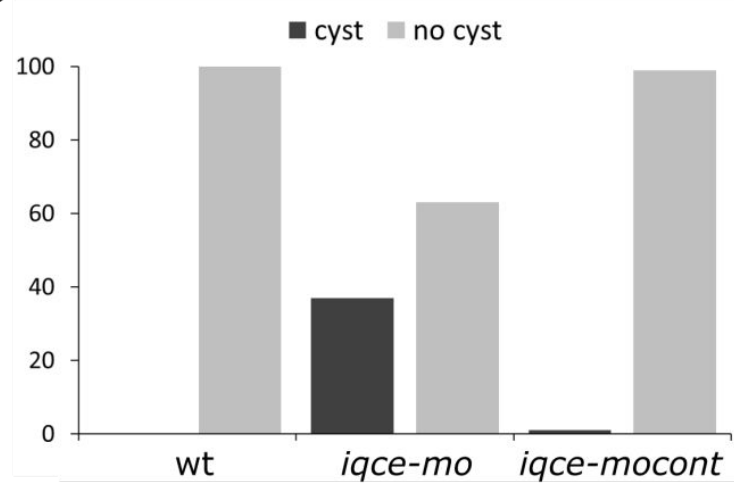

$\mathrm{E}$

$\mathrm{F}$
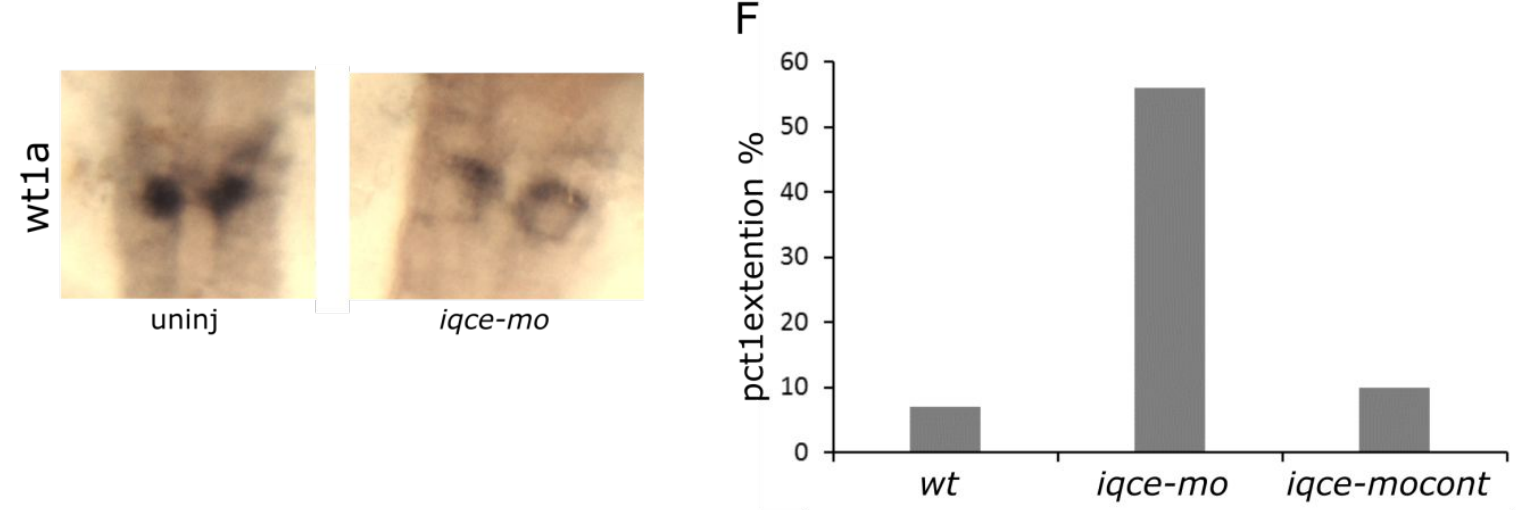

Supplementary Figure S3. Efficacy of iqce morpholinos. A. Morpholinos directed against the start codon of iqce blocks the expression of a construct containing iqce cDNA in frame with gfp (central panel). In contrast, 5 mismatch morpholinos did not reduce expression of the chimeric protein (left panel) compared to iqce-gfp alone (right panel). $24 \mathrm{hpf}$. B. in situ hybridization showing iqce expression pattern in 10 somites, 20 and 48 hpf embryos. Arrow indicates the pronephric duct. $\mathrm{F}$ : forebrain ventricule; $\mathrm{H}$ : hindbrain ventricule; $\mathrm{M}$ : midbrain ventricule. C. Chart showing the percentage of curved embryos in uninjected $(n=200)$, iqce- 
mo $(\mathrm{n}=120)$; and iqce-mocont $(\mathrm{n}=221)$ injected embryos. $48 \mathrm{hpf}$, data includes $\pm \mathrm{SD}$ and statistical significance was determined using the Student's t-test, all comparisons were significant with a p-value<0.05. D. Chart showing the percentage of cysts in uninjected $(\mathrm{n}=101)$, iqce-mo $(\mathrm{n}=70)$ and iqce-mocont $(\mathrm{n}=90)$ injected embryos. Statistical significance was determined using the Student's t-test, all comparisons were significant with a p-value<0.05. E. wt1a in situ hybridization showing expression within glomeruli in non-injected embryos and at the periphery of the cyst in morphants (48 hpf). F. Chart showing the percentage of embryos with ptc1 extension in 10 somites embryos $(n=191)$. 
Supplementary Table S1. Summary of the whole exome sequencing results. SNV: Single nucleotide variation, indel: gain or loss of up to 50 nucleotides at a single locus, SV: Structural Variation. Annotations are gathered using Alamut Batch v1.11, especially for the variation databases including gnomAD (v2.0.2, Oct. 2017), 1000 Genomes Project phase3 release (version 20150813 v5b) and the following predictions including phastCons (UCSC, 44 vertebrates). Effect on the splice has been evaluated using the MaxEntScan (Yeo \& Burge, 2004), NNSPLICE 0.9 (Reese, Eeckman, Kulp, \& Haussler, 1997) and Splice Site Finder (Shapiro \& Senapathy, 1987) by calculating score change between the wild type and the mutated sequences expressed as a percent differences. Missenses have been evaluated using default parameters from PolyPhen-2 (2.2.2) (Adzhubei et al., 2010) and SIFT 4.0.3 (Kumar, Henikoff, \& Ng, 2009). Default cut-offs used have been described in VaRank (Geoffroy et al., 2015) for both type of predictions. Exclusion of SV with a DGV (Gold standard from 20160515) frequency $>1 \%$ is done only with studies of more than 1000 individuals.

\begin{tabular}{|c|c|c|c|c|c|c|c|c|c|c|c|c|}
\hline & \multicolumn{3}{|c|}{ A.IV-1 } & \multicolumn{3}{|c|}{ B.II-2 } & \multicolumn{3}{|c|}{ B.II-3 } & \multicolumn{3}{|c|}{ C.IV-4 } \\
\hline & SNV & indel & sv & SNV & indel & sv & SNV & Indel & sv & SNV & Indel & sv \\
\hline Total number of variants & 83129 & 11664 & 31 & 81972 & 11319 & 17 & 82116 & 11195 & 16 & 78906 & 11727 & 31 \\
\hline $\begin{array}{l}\text { After exclusion of variants with an allele frequency }>1 \% \\
\text { (gnomAD, 1000G, internal exome database, DGV) }\end{array}$ & 9771 & 1125 & 16 & 7069 & 993 & 6 & 6856 & 943 & 9 & 5323 & 982 & 17 \\
\hline $\begin{array}{l}\text { After exclusion of SNV/indel in 5'UTR, 3'UTR, downstream, } \\
\text { upstream, intron and synonymous locations without local splice } \\
\text { effect prediction }\end{array}$ & 788 & 76 & 1 & 553 & 59 & 1 & 560 & 65 & 1 & 753 & 113 & 1 \\
\hline $\begin{array}{l}\text { After exclusion of missense with no deleterious effect } \\
\text { according to SIFT, PPH2 or PhastCons }\end{array}$ & 669 & 76 & 1 & 458 & 59 & 1 & 465 & 65 & 1 & 641 & 113 & 1 \\
\hline $\begin{array}{l}\text { After exclusion of variants not in a ciliary genes list (Nevers et } \\
\text { al., 2017) and not in non-syndromic polydactyly known genes }\end{array}$ & 52 & 6 & 1 & 31 & 2 & 2 & 40 & 4 & 2 & 78 & 15 & 1 \\
\hline $\begin{array}{l}\text { After selection of variants consistent with recessive } \\
\text { transmission (compound heterozygous, homozygous variants) }\end{array}$ & $\begin{array}{r}7 \text { hom. } \\
\text { SHA } \\
3 \text { comp. } \mathrm{h}\end{array}$ & $\begin{array}{l}\text { IAAF1, IC } \\
\text { TULP1 } \\
\text { ARMC3, }\end{array}$ & $\begin{array}{l}1 A D D \\
111, M G A\end{array}$ & & $\begin{array}{l}0 \mathrm{hom} \\
1 \mathrm{com}\end{array}$ & $\begin{array}{l}\text { ozyg } \\
\text { ip. he }\end{array}$ & $\begin{array}{l}\text { ous vari } \\
\text { et. In } I Q C\end{array}$ & iant & & $\begin{array}{r}3 \text { hom.: } \\
11 \mathrm{com} \\
\text { DNAH7 } \\
\text { LRRCC1, } \\
\text { SMA }\end{array}$ & $\begin{array}{l}\text { T1, AGTF } \\
\text { et: CTDS } \\
\text { 'NLL1, EF } \\
\text { ?C46, MR } \\
\text { I5, TMEM }\end{array}$ & $\begin{array}{l}\text { KDM4C } \\
D D X 1, \\
\text { IFT20, } \\
\text { PRKDC, } \\
\text { PTE }\end{array}$ \\
\hline
\end{tabular}


Supplementary Table S2. Homozygous regions identified in individual A.IV-1.

\begin{tabular}{|c|c|c|c|c|c|c|c|c|}
\hline Chr & SNP1 & SNP2 & POS1 & POS2 & MB & \#SNP & DENSITY & \\
\hline 1 & rs4147830 & rs11431894 & 94544276 & 100680594 & 6.14 & 33 & 185.949 & \\
\hline 1 & rs4915053 & rs3761934 & 108113526 & 114189067 & 6.08 & 136 & 44.673 & \\
\hline 1 & 1_148741720_T_C & rs11428742 & 148741720 & 150550020 & 1.81 & 22 & 82.196 & \\
\hline 1 & rs4636 & rs10927011 & 234744413 & 243579112 & 8.83 & 125 & 70.678 & \\
\hline 2 & rs7577088 & rs3764906 & 39233500 & 42588195 & 3.35 & 20 & 167.735 & \\
\hline 2 & rs522893 & 2_188250301_T_A & 169690729 & 188250301 & 18.56 & 224 & 82.855 & \\
\hline 2 & rs2304704 & rs12477076 & 190430177 & 191848312 & 1.42 & 23 & 61.658 & \\
\hline 3 & rs901854 & rs113574694 & 4856234 & 10089550 & 5.23 & 58 & 90.230 & \\
\hline 3 & rs9814557 & rs2246945 & 135720540 & 137843476 & 2.12 & 21 & 101.092 & \\
\hline 4 & rs13146318 & rs1051447 & 41615690 & 49063872 & 7.45 & 68 & 109.532 & \\
\hline 4 & rs4864727 & rs12621 & 54011424 & 57897570 & 3.89 & 61 & 63.707 & \\
\hline 4 & rs10434219 & rs344122 & 62598689 & 77637502 & 15.04 & 175 & 85.936 & \\
\hline 6 & rs2076506 & 6_32485853_C_T & 22570064 & 32485853 & 9.92 & 646 & 15.350 & \\
\hline 6 & rs1059547 & rs12189627 & 32557529 & 41059458 & 8.50 & 309 & 27.514 & TULP1 \\
\hline 7 & rs4916935 & rs798479 & 197267 & 2691201 & 2.49 & 41 & 60.828 & IQCE \\
\hline 7 & rs7794563 & rs80024169 & 5347295 & 14775824 & 9.43 & 78 & 120.879 & \\
\hline 7 & rs4718101 & rs6460315 & 64152353 & 66289040 & 2.14 & 38 & 56.229 & \\
\hline 8 & rs3989699 & rs13271468 & 7218621 & 12236202 & 5.02 & 132 & 38.012 & \\
\hline 8 & 8_12273335_G_A & rs66480340 & 12273335 & 24211885 & 11.94 & 202 & 59.102 & \\
\hline 8 & rs10086200 & rs59331088 & 61193588 & 74005131 & 12.81 & 72 & 177.938 & \\
\hline 8 & rs11777189 & rs12545587 & 99057150 & 136560952 & 37.50 & 204 & 183.842 & \\
\hline 9 & rs4977555 & rs11515 & 19550308 & 21968199 & 2.42 & 30 & 80.596 & \\
\hline 9 & rs7863560 & rs3217100 & 119495697 & 123734288 & 4.24 & 30 & 141.286 & \\
\hline 11 & 11_32452000_GG_- & rs2076623 & 32452000 & 33564123 & 1.11 & 25 & 44.485 & \\
\hline 11 & rs12274095 & rs7101792 & 69063767 & 71249386 & 2.19 & 28 & 78.058 & \\
\hline 11 & rs3740912 & rs4937391 & 125891269 & 128786294 & 2.90 & 32 & 90.470 & \\
\hline 12 & rs11054683 & rs1600 & 12247616 & 14656768 & 2.41 & 31 & 77.715 & \\
\hline 12 & rs3088008 & rs3210837 & 49724955 & 51685831 & 1.96 & 55 & 35.652 & \\
\hline 12 & rs74651927 & rs2242497 & 52695754 & 56992630 & 4.30 & 185 & 23.226 & \\
\hline 12 & rs1663564 & rs7488309 & 105546172 & 118520170 & 12.97 & 152 & 85.355 & \\
\hline 12 & rs1794956 & rs11059841 & 124848137 & 129190021 & 4.34 & 37 & 117.348 & \\
\hline 14 & rs34800262 & rs2249922 & 51370852 & 52906081 & 1.54 & 34 & 45.154 & \\
\hline 14 & rs2297113 & rs863091 & 58036494 & 59112475 & 1.08 & 23 & 46.782 & \\
\hline 14 & rs35533709 & rs2057482 & 60433392 & 62213848 & 1.78 & 30 & 59.349 & \\
\hline 15 & rs4923929 & rs555001 & 42439376 & 43545728 & 1.11 & 37 & 29.901 & \\
\hline 15 & rs11351249 & rs2623989 & 72105929 & 73545732 & 1.44 & 23 & 62.600 & \\
\hline 15 & rs752270 & rs4777755 & 87099537 & 93510603 & 6.41 & 171 & 37.492 & \\
\hline 16 & rs6564764 & rs931713 & 80581631 & 85023855 & 4.44 & 121 & 36.713 & \\
\hline 17 & rs8071836 & rs67749219 & 47238028 & 49711042 & 2.47 & 40 & 61.825 & \\
\hline
\end{tabular}


Supplementary Table S3. List and characteristics of primers used in this study

\begin{tabular}{|c|c|c|c|c|c|}
\hline Application & Gene & Exon & Forward (5'-3') & Reverse (5'-3') & $\begin{array}{l}\text { Size } \\
\text { (bp) }\end{array}$ \\
\hline \multirow[t]{4}{*}{ DNA } & IQCE & 12 & СTCTGAGCTCACAACCAACAG & GTTGCCCAAGGGTTCACA & 399 \\
\hline & & 16 & TGGTTTCTGGTGTTGTCAGG & TTTTCAGTGGCTGGTCAGAA & 382 \\
\hline & ATP6V1B1 & 3 & AGCCGTGGGAAGTAGGTGTT & ATGTCCCATTGACCCCACT & 385 \\
\hline & TULP1 & $12 / 13$ & GGGATGTAGGATCCCСTCA & CATGCCAGGAATGATGACG & 481 \\
\hline \multirow[t]{17}{*}{ cDNA } & $I Q C E$ & $3-4 / 5$ & ACATCGCCAAAGTCACCTTATC & AGGGCCTGGGTCAGACTT & 158 \\
\hline & GSTT1 & $2-3 / 3$ & ACCTTGACGGAGAGTGTGG & GCAGCTTCTCCGCAGAGT & 144 \\
\hline & SLC30A8 & $9-10 / 10$ & CCATCTTACTCATGGAAGGTGTG & GCTGTAGCAACATGAGCTGAGA & 154 \\
\hline & RSPO1 & $4 / 5$ & TGGAGAGGAACGACATCCG & CCTTACACTTGGTGCAGAAGTTA & 156 \\
\hline & FGF9 & $2 / 3$ & GGCCTGGTCAGCATTCGAG & GTATCGCCTTCCAGTGTCCAC & 180 \\
\hline & SFRP1 & $1 / 2$ & GCTTCTACTGGCCCGAGATG & TGGCCTCAGATTTCAACTCGT & 180 \\
\hline & MECOM & $4 / 5$ & AAACTCGAAAGCGAGAATGATCT & TGGTGGCGAATTAAATTGGACTT & 185 \\
\hline & $A L P L$ & $6-7 / 7$ & AACATCAGGGACATTGACGTG & GTATCTCGGTTTGAAGCTCTTCC & 159 \\
\hline & IGF2 & $2-3 / 3-4$ & TTCTACTTCAGCAGGCCCG & GAAGTTGTCCGGAAGCACG & 168 \\
\hline & GAPDH & $4-5 / 6$ & GGAGCGAGATCCCTCCAAAAT & GGCTGTTGTCATACTTCTCATGG & 197 \\
\hline & HPRT1 & $1-2 / 2-3$ & CCTGGCGTCGTGATTAGTGAT & AGACGTTCAGTCCTGTCCATAA & 131 \\
\hline & EFCAB7 & $6 / 6-7$ & GTCAGCAACCAGGAAGTTCA & CATGTGTTGCCAGTCCTTTATT & 115 \\
\hline & EVC2 & $6-7 / 8$ & CTCGTCACGGAACAGAACAC & CAGGTCAGCACAAGGGAGAG & 135 \\
\hline & EVC & $2 / 4$ & TCGAAGGACAAGGAAGCTGT & TTTCATGCAGAGACGGGTTG & 148 \\
\hline & GLI2 & $2 / 3$ & AAGCAAGAAGCCAAAAGTGG & TGGTACCTTCCTTCCTGGTG & 188 \\
\hline & GL/3 & $2 / 3$ & GGCATTTTTGGTCGAAGAGA & GGACATTCTGTGGCTGCATA & 238 \\
\hline & SMO & $2 / 3$ & TGCCCAAGTGTGAGAATGAC & TACCAGCTCTTGGGGTTGTC & 229 \\
\hline
\end{tabular}


Supplementary Table S4. Comparison of haplotypes of individuals A.IV-1 and B.II-2.

\begin{tabular}{|c|c|c|c|c|c|c|c|}
\hline ID & Gene & Chr & Position & Allele_1_A.IV-1 & Allele_2_A.IV-1 & $\begin{array}{l}\text { Allele_1_II- } \\
\text { 2_Family2 }\end{array}$ & $\begin{array}{l}\text { Allele_1_II- } \\
\text { 2_Family2 }\end{array}$ \\
\hline 7_2255974_C_T & MAD1L1 & 7 & 2255974 & $\mathrm{C}$ & C & C & $\mathrm{T}$ \\
\hline 7_2256017_C_A & MAD1L1 & 7 & 2256017 & $\mathrm{C}$ & C & C & $A$ \\
\hline 7_2257612_G_A & MAD1L1 & 7 & 2257612 & $A$ & $A$ & A & $G$ \\
\hline 7_2259134_C_T & MAD1L1 & 7 & 2259134 & C & C & C & $\mathrm{T}$ \\
\hline 7_2260715_G_T & MAD1L1 & 7 & 2260715 & $\mathrm{~T}$ & $\mathrm{~T}$ & $\mathrm{~T}$ & $\mathrm{~T}$ \\
\hline 7_2269552_G_T & MAD1L1 & 7 & 2269552 & $\mathrm{~T}$ & $\mathrm{~T}$ & $\mathrm{~T}$ & $G$ \\
\hline 7_2278663_T_A & FTSJ2 & 7 & 2278663 & $\mathrm{~T}$ & $\mathrm{~T}$ & A & A \\
\hline 7_2279482_A_C & FTSJ2 & 7 & 2279482 & $A$ & $A$ & $\mathrm{C}$ & C \\
\hline 7_2279851_T_C & FTSJ2 & 7 & 2279851 & $\mathrm{~T}$ & $\mathrm{~T}$ & C & C \\
\hline 7_2281754_C_T & FTSJ2 & 7 & 2281754 & C & C & C & $\mathrm{T}$ \\
\hline 7_2290328_C_T & SNX8 & 7 & 2290328 & C & C & C & $\mathrm{T}$ \\
\hline 7_2290336_T_G & $S N X 8$ & 7 & 2290336 & $\mathrm{~T}$ & $\mathrm{~T}$ & $\mathrm{~T}$ & G \\
\hline 7_2290372_C_T & $S N X 8$ & 7 & 2290372 & C & C & $\mathrm{T}$ & $\mathrm{T}$ \\
\hline 7_2290522_C_T & SNX8 & 7 & 2290522 & $\mathrm{~T}$ & $\mathrm{~T}$ & C & C \\
\hline 7_2292881_-_TG & $S N X 8$ & 7 & 2292881 & - & - & TG & TG \\
\hline 7_2296493_A_G & SNX8 & 7 & 2296493 & G & G & G & $G$ \\
\hline 7_2297006_A_G & SNX8 & 7 & 2297006 & A & A & G & G \\
\hline 7_2303000_G_A & $S N X 8$ & 7 & 2303000 & $A$ & $A$ & G & G \\
\hline 7_2303109_G_A & $S N X 8$ & 7 & 2303109 & G & G & G & $A$ \\
\hline 7_2303986_T_C & SNX8 & 7 & 2303986 & C & C & C & C \\
\hline 7_2304191_A_C & $S N X 8$ & 7 & 2304191 & A & A & C & C \\
\hline 7_2309159_-_A & SNX8 & 7 & 2309159 & - & - & - & A \\
\hline 7_2309174_G_A & SNX8 & 7 & 2309174 & G & G & G & A \\
\hline 7_2309185_G_A & SNX8 & 7 & 2309185 & G & G & G & $A$ \\
\hline 7_2309209_C_T & SNX8 & 7 & 2309209 & C & C & C & $\mathrm{T}$ \\
\hline 7_2314652_G_A & $S N X 8$ & 7 & 2314652 & G & G & G & A \\
\hline 7_2315008_G_C & SNX8 & 7 & 2315008 & G & G & C & C \\
\hline 7_2318254_ACTC_- & SNX8 & 7 & 2318254 & - & - & АСTC & АСTC \\
\hline 7_2318268_C_T & SNX8 & 7 & 2318268 & $\mathrm{~T}$ & $\mathrm{~T}$ & C & $\mathrm{C}$ \\
\hline 7_2318463_C_T & SNX8 & 7 & 2318463 & C & $\mathrm{T}$ & C & C \\
\hline 7_2318472_C_A & SNX8 & 7 & 2318472 & C & $A$ & C & C \\
\hline 7_2349470_T_C & $S N X 8$ & 7 & 2349470 & $\mathrm{~T}$ & $\mathrm{~T}$ & $\mathrm{~T}$ & C \\
\hline 7_2394991_C_T & EIF3B & 7 & 2394991 & $\mathrm{C}$ & C & C & $\mathrm{T}$ \\
\hline 7_2400306_T_C & EIF3B & 7 & 2400306 & C & C & C & $\mathrm{T}$ \\
\hline 7_2405885_C_A & EIF3B & 7 & 2405885 & A & A & A & C \\
\hline 7_2409035_C_T & EIF3B & 7 & 2409035 & $\mathrm{C}$ & C & C & $\mathrm{T}$ \\
\hline 7_2414142_A_G & EIF3B & 7 & 2414142 & G & G & G & A \\
\hline 7_2414365_A_G & EIF3B & 7 & 2414365 & A & A & A & G \\
\hline 7_2416822_A_G & EIF3B & 7 & 2416822 & $A$ & A & A & G \\
\hline 7_2418645_A_G & EIF3B & 7 & 2418645 & A & A & A & G \\
\hline 7_2433729_C_A & NA & 7 & 2433729 & C & C & C & A \\
\hline 7_2434522_G_A & NA & 7 & 2434522 & G & G & $A$ & A \\
\hline 7_2434596_TT_- & NA & 7 & 2434596 & TT & TT & TT & - \\
\hline 7_2434597_T_- & NA & 7 & 2434597 & $\mathrm{~T}$ & $\mathrm{~T}$ & $\mathrm{~T}$ & - \\
\hline
\end{tabular}




\begin{tabular}{|c|c|c|c|c|c|c|c|}
\hline 7_2472429_C_A & CHST12 & 7 & 2472429 & $\mathrm{C}$ & $\mathrm{C}$ & $\mathrm{C}$ & $A$ \\
\hline 7_2472455_A_T & CHST12 & 7 & 2472455 & A & $A$ & $A$ & $\mathrm{~T}$ \\
\hline 7_2489311_C_G & NA & 7 & 2489311 & C & C & C & G \\
\hline $\begin{array}{l}\text { 7_2489336_GTGTGT } \\
\text { GCACCT_- }\end{array}$ & NA & 7 & 2489336 & GTGTGTGCACCT & GTGTGTGCACCT & GTGTGTGCACCT & - \\
\hline 7_2489382_A_G & NA & 7 & 2489382 & A & A & A & G \\
\hline 7_2515382_-_C & NA & 7 & 2515382 & - & - & C & $\mathrm{C}$ \\
\hline 7_2517473_C_T & NA & 7 & 2517473 & C & $\mathrm{C}$ & $\mathrm{C}$ & $\mathrm{T}$ \\
\hline 7_2552986_A_C & LFNG & 7 & 2552986 & A & $A$ & $A$ & $\mathrm{C}$ \\
\hline 7_2553030_T_C & LFNG & 7 & 2553030 & $\mathrm{~T}$ & $\mathrm{~T}$ & $\mathrm{~T}$ & $\mathrm{C}$ \\
\hline 7_2557266_T_C & LFNG & 7 & 2557266 & $\mathrm{~T}$ & $\mathrm{~T}$ & C & C \\
\hline 7_2557377_T_C & LFNG & 7 & 2557377 & $\mathrm{~T}$ & $\mathrm{~T}$ & $\mathrm{~T}$ & $\mathrm{C}$ \\
\hline 7_2565268_C_A & LFNG/MIR4648 & 7 & 2565268 & C & C & $A$ & A \\
\hline 7_2566433_G_A & LFNG/MIR4648 & 7 & 2566433 & G & G & G & $A$ \\
\hline 7_2577691_C_G & BRAT1 & 7 & 2577691 & C & C & C & $\mathrm{G}$ \\
\hline 7_2577781_T_C & BRAT1 & 7 & 2577781 & $\mathrm{~T}$ & $\mathrm{~T}$ & $\mathrm{~T}$ & C \\
\hline 7_2578237_T_C & BRAT1 & 7 & 2578237 & $\mathrm{~T}$ & $\mathrm{~T}$ & $\mathrm{~T}$ & C \\
\hline 7_2578238_C_T & BRAT1 & 7 & 2578238 & C & C & C & $\mathrm{T}$ \\
\hline 7_2578434_G_T & BRAT1 & 7 & 2578434 & G & G & $G$ & $\mathrm{~T}$ \\
\hline 7_2578455_G_A & BRAT1 & 7 & 2578455 & G & $\mathrm{G}$ & G & A \\
\hline 7_2580914_C_T & BRAT1 & 7 & 2580914 & $\mathrm{C}$ & $\mathrm{C}$ & $\mathrm{C}$ & $\mathrm{T}$ \\
\hline 7_2581994_T_C & BRAT1 & 7 & 2581994 & $\mathrm{~T}$ & $\mathrm{~T}$ & $\mathrm{~T}$ & $\mathrm{C}$ \\
\hline $\begin{array}{l}7{ }^{7} 2582154{ }^{-} \\
\text {CCCCAGCTCCCG } \\
\text { GGTGT }\end{array}$ & BRAT1 & 7 & 2582154 & $\begin{array}{l}\text { CCCCAGCCTCC } \\
\text { CGGGTGT }\end{array}$ & $\begin{array}{c}\text { CCCCAGCCTCC } \\
\text { CGGGTGT }\end{array}$ & - & - \\
\hline 7_2582233_A_G & BRAT1 & 7 & 2582233 & A & $A$ & $A$ & G \\
\hline 7_2582381_T_A & BRAT1 & 7 & 2582381 & $\mathrm{~T}$ & $\mathrm{~T}$ & $\mathrm{~T}$ & $A$ \\
\hline 7_2582584_G_A & BRAT1 & 7 & 2582584 & G & G & G & $A$ \\
\hline 7_2583165_G_A & BRAT1 & 7 & 2583165 & G & G & G & A \\
\hline 7_2583328_C_T & BRAT1 & 7 & 2583328 & $\mathrm{C}$ & $\mathrm{C}$ & $\mathrm{C}$ & $\mathrm{T}$ \\
\hline 7_2584523_T_C & BRAT1 & 7 & 2584523 & $\mathrm{~T}$ & $\mathrm{~T}$ & $\mathrm{~T}$ & $\mathrm{C}$ \\
\hline 7_2587122_T_C & BRAT1 & 7 & 2587122 & $\mathrm{~T}$ & $\mathrm{~T}$ & $\mathrm{~T}$ & $\mathrm{C}$ \\
\hline 7_2598757_T_C & IQCE & 7 & 2598757 & $\mathrm{~T}$ & $\mathrm{~T}$ & $\mathrm{~T}$ & C \\
\hline 7_2604768_GTGT_- & $I Q C E$ & 7 & 2604768 & GTGT & GTGT & GTGT & - \\
\hline 7_2604769_T_* & IQCE & 7 & 2604769 & $\mathrm{~T}$ & $\mathrm{~T}$ & $\mathrm{~T}$ & * \\
\hline 7_2604816_C_T & $I Q C E$ & 7 & 2604816 & C & C & $\mathrm{C}$ & $\mathrm{T}$ \\
\hline 7_2611878_T_C & IQCE & 7 & 2611878 & $\mathrm{~T}$ & $\mathrm{~T}$ & $\mathrm{~T}$ & $\mathrm{C}$ \\
\hline 7_2612225_G_A & IQCE & 7 & 2612225 & A & $A$ & G & $G$ \\
\hline 7_2613042_G_C & $I Q C E$ & 7 & 2613042 & G & G & G & C \\
\hline 7_2618070_-_A & $I Q C E$ & 7 & 2618070 & - & - & - & $A$ \\
\hline 7_2619341_GT_- & IQCE & 7 & 2619341 & GT & GT & GT & - \\
\hline 7_2619363_G_C & $I Q C E$ & 7 & 2619363 & G & G & G & $\mathrm{C}$ \\
\hline 7_2623351_G_A & $I Q C E$ & 7 & 2623351 & G & G & G & $A$ \\
\hline 7_2623801_G_T & $I Q C E$ & 7 & 2623801 & G & G & G & $\mathrm{T}$ \\
\hline $\begin{array}{l}\text { 7_2625907_TGTCCC } \\
\text { GGAG_- }\end{array}$ & $I Q C E$ & 7 & 2625907 & - & - & - & TGTCCCGGAG \\
\hline $\begin{array}{l}\text { 7_2626039_CAGGGA } \\
\text { ATGG_- }\end{array}$ & $I Q C E$ & 7 & 2626039 & CAGGGAATGG & CAGGGAATGG & CAGGGAATGG & - \\
\hline 7_2627310_T_A & IQCE & 7 & 2627310 & $\mathrm{~T}$ & $\mathrm{~T}$ & $\mathrm{~T}$ & $A$ \\
\hline 7_2627579_AG_- & $I Q C E$ & 7 & 2627579 & $A G$ & $A G$ & - & - \\
\hline 7_2632477_A_G & IQCE & 7 & 2632477 & $A$ & $A$ & $A$ & $\mathrm{G}$ \\
\hline
\end{tabular}




$\begin{array}{llllllll}\text { 7_2634518_AGAG_- } & \text { IQCE } & 7 & 2634518 & \text { AGAG } & \text { AGAG } & \text { AGAG } & \text { - } \\ \text { 7_2636689_C_T } & \text { IQCE } & 7 & 2636689 & \text { C } & \text { C } & \text { C } & \text { T } \\ \text { 7_2638347_C_G } & \text { IQCE } & 7 & 2638347 & \text { C } & \text { C } & \text { C } & \text { G } \\ \text { 7_2640985_G_C } & \text { IQCE } & 7 & 2640985 & \text { G } & \text { G } & \text { G } & \text { C } \\ \text { 7_2647048_C_G } & \text { IQCE } & 7 & 2647048 & \text { C } & \text { C } & \text { C } & \text { G } \\ \text { 7_2691201_C_A } & \text { TTYH3 } & 7 & 2691201 & \text { A } & \text { A } & \text { C } & \text { C } \\ \text { 7_2699687_-G } & \text { TTYH3 } & 7 & 2699687 & - & - & \text { G } & \text { G } \\ \text { 7_2701809_T_C } & \text { TTYH3 } & 7 & 2701809 & \text { T } & \text { C } & \text { T } & \text { T } \\ \text { 7_2742097_T_C } & \text { AMZ1 } & 7 & 2742097 & \text { C } & \text { C } & \text { T } & \text { T } \\ \text { 7_2749565_G_A } & \text { AMZ1 } & 7 & 2749565 & \text { G } & \text { G } & \text { G } & \text { A } \\ \text { 7_2752152_G_A } & \text { AMZ1 } & 7 & 2752152 & \text { G } & \text { G } & \text { G } & \text { A } \\ \text { 7_2752487_G_A } & \text { AMZ1 } & 7 & 2752487 & \text { A } & \text { A } & \text { G } & \text { G } \\ \text { 7_2773035_-_AT } & \text { GNA12 } & 7 & 2773035 & - & - & \text { AT } & \text { AT } \\ \text { 7_2802173_TC } & \text { GNA12 } & 7 & 2802173 & \text { T } & \text { T } & \text { T } & \text { C } \\ \text { 7_2802522_T_C } & \text { GNA12 } & 7 & 2802522 & \text { T } & \text { T } & \text { T } & \text { C } \\ \text { 7_2834869_C_T } & \text { GNA12 } & 7 & 2834869 & \text { C } & \text { C } & \text { T } & \text { T } \\ \text { 7_2854010_G_A } & \text { GNA12 } & 7 & 2854010 & \text { G } & \text { G } & \text { A } & \text { A } \\ \text { 7_2946461_T_C } & \text { CARD11 } & 7 & 2946461 & \text { C } & \text { C } & \text { C } & \text { T } \\ \text { 7_2952912_G_A } & \text { CARD11 } & 7 & 2952912 & \text { G } & \text { G } & \text { G } & \text { A } \\ \text { 7_2957005_TCC } & \text { CARD11 } & 7 & 2957005 & \text { C } & \text { C } & \text { C } & \text { T } \\ \text { 7_2958298_C_T } & \text { CARD11 } & 7 & 2958298 & \text { C } & \text { C } & \text { T } & \text { T } \\ \text { 7_2962241_G_A } & \text { CARD11 } & 7 & 2962241 & \text { A } & \text { A } & \text { G } & \text { G } \\ \text { 7_2962753_G_A } & \text { CARD11 } & 7 & 2962753 & \text { G } & \text { G } & \text { G } & \text { A } \\ \text { 7_2966445_TG } & \text { CARD11 } & 7 & 2966445 & \text { T } & \text { T } & \text { T } & \text { G } \\ \text { 7_2966466_GT_- } & \text { CARD11 } & 7 & 2966466 & \text { GT } & \text { GT } & \text { GT } & \text { - } \\ \text { 7_2968195_G_A } & \text { CARD11 } & 7 & 2968195 & \text { G } & \text { G } & \text { G } & \text { A } \\ \text { 7_2968361_G_A } & \text { CARD11 } & 7 & 2968361 & \text { G } & \text { G } & \text { G } & \text { A } \\ \text { 7_2968486_G_C } & \text { CARD11 } & 7 & 2968486 & \text { G } & \text { G } & \text { G } & \text { C } \\ \text { 7_2985364_AC_- } & \text { CARD11 } & 7 & 2985364 & \text { A } & \text { C } & \text { - } & \text { - }\end{array}$


Supplementary Table S5. Differentially expressed transcripts in A.IV-1 fibroblasts.

\begin{tabular}{|c|c|c|c|c|c|}
\hline Gene name & Ensembl gene id & Description & $\log 2(\mathrm{FC})$ & P-value & Adjusted p-value \\
\hline$A B C A 8$ & ENSG00000141338 & $\begin{array}{l}\text { ATP-binding cassette, sub-family A } \\
\text { (ABC1), member } 8\end{array}$ & -4.205829134 & $3.52 \mathrm{E}-05$ & 0.019362186 \\
\hline AC011294.3 & ENSG00000233539 & Uncharacterized protein & 3.130226421 & $2.55 \mathrm{E}-07$ & 0.000427239 \\
\hline$A C C S$ & ENSG00000110455 & $\begin{array}{l}\text { 1-aminocyclopropane-1-carboxylate } \\
\text { synthase homolog (Arabidopsis)(non- } \\
\text { functional) }\end{array}$ & -0.953037549 & $1.28 \mathrm{E}-07$ & 0.00025011 \\
\hline ADAMTSL4 & ENSG00000143382 & ADAMTS-like 4 & -3.153983754 & $5.00 \mathrm{E}-07$ & 0.000732904 \\
\hline$A L D H 1 A 1$ & ENSG00000165092 & $\begin{array}{l}\text { aldehyde dehydrogenase } 1 \text { family, } \\
\text { member } \mathrm{A} 1\end{array}$ & -6.206001924 & 1.99E-08 & $5.40 \mathrm{E}-05$ \\
\hline$A L P L$ & ENSG00000162551 & $\begin{array}{l}\text { alkaline phosphatase, } \\
\text { liver/bone/kidney }\end{array}$ & -3.039649649 & 0.000113858 & 0.038536558 \\
\hline APBA2 & ENSG00000034053 & $\begin{array}{l}\text { amyloid beta (A4) precursor protein- } \\
\text { binding, family A, member } 2\end{array}$ & -2.732442847 & $6.79 E-05$ & 0.028790694 \\
\hline ARHGAP28 & ENSG00000088756 & Rho GTPase activating protein 28 & 2.800539497 & $1.58 \mathrm{E}-05$ & 0.010684692 \\
\hline C10orf54 & ENSG00000107738 & $\begin{array}{l}\text { chromosome } 10 \text { open reading frame } \\
54\end{array}$ & -1.64124697 & $9.82 E-05$ & 0.035643913 \\
\hline $\begin{array}{l}\text { C1QTNF9B- } \\
\text { AS1 }\end{array}$ & ENSG00000205861 & C1QTNF9B antisense RNA 1 & -2.047191404 & $3.88 \mathrm{E}-05$ & 0.019780947 \\
\hline$C 2 C D 4 A$ & ENSG00000198535 & $\begin{array}{l}\text { C2 calcium-dependent domain } \\
\text { containing 4A }\end{array}$ & 5.96982663 & 8.95E-07 & 0.001085914 \\
\hline$C 4 A$ & ENSG00000244731 & $\begin{array}{l}\text { complement component 4A (Rodgers } \\
\text { blood group) }\end{array}$ & -6.329532092 & $5.21 \mathrm{E}-11$ & $3.66 \mathrm{E}-07$ \\
\hline$C C D C 180$ & ENSG00000197816 & coiled-coil domain containing 180 & 2.351169896 & $3.81 \mathrm{E}-05$ & 0.019780947 \\
\hline$C D 200$ & ENSG00000091972 & CD200 molecule & -4.053753384 & 0.000135641 & 0.044622222 \\
\hline CHI3L1 & ENSG00000133048 & $\begin{array}{l}\text { chitinase 3-like } 1 \text { (cartilage } \\
\text { glycoprotein-39) }\end{array}$ & 4.852100597 & $6.43 \mathrm{E}-07$ & 0.000837885 \\
\hline CHN1 & ENSG00000128656 & chimerin 1 & -1.617295158 & 2.25E-09 & $1.32 \mathrm{E}-05$ \\
\hline CHRDL2 & ENSG00000054938 & chordin-like 2 & -4.241249176 & 0.000101018 & 0.03591767 \\
\hline CNTN1 & ENSG00000018236 & contactin 1 & -4.135254047 & 8.76E-05 & 0.032879001 \\
\hline COL11A1 & ENSG00000060718 & collagen, type XI, alpha 1 & -4.558619592 & $6.30 \mathrm{E}-05$ & 0.027357715 \\
\hline CSF2RA & ENSG00000198223 & $\begin{array}{l}\text { colony stimulating factor } 2 \text { receptor, } \\
\text { alpha, low-affinity (granulocyte- } \\
\text { macrophage) }\end{array}$ & 3.072895701 & $6.90 \mathrm{E}-05$ & 0.028906369 \\
\hline CYP21A1P & ENSG00000204338 & $\begin{array}{l}\text { cytochrome P450, family } 21 \text {, subfamily } \\
\text { A, polypeptide } 1 \text { pseudogene }\end{array}$ & -4.61429374 & $6.95 \mathrm{E}-06$ & 0.005439088 \\
\hline$D U S P 2$ & ENSG00000158050 & dual specificity phosphatase 2 & 3.527490256 & $1.70 \mathrm{E}-05$ & 0.01128586 \\
\hline ECM1 & ENSG00000143369 & extracellular matrix protein 1 & -1.261675626 & 5.81E-07 & 0.000786019 \\
\hline$E M C N$ & ENSG00000164035 & endomucin & 3.361027658 & 4.95E-05 & 0.0229427 \\
\hline EYA4 & ENSG00000112319 & eyes absent homolog 4 (Drosophila) & -4.200503943 & 4.26E-05 & 0.020550766 \\
\hline$F A M 21 B$ & ENSG00000152726 & $\begin{array}{l}\text { family with sequence similarity } 21, \\
\text { member B }\end{array}$ & -4.995162913 & 7.93E-08 & 0.000164108 \\
\hline FGF9 & ENSG00000102678 & fibroblast growth factor 9 & 4.555259473 & 5.95E-08 & 0.000133394 \\
\hline$F O S B$ & ENSG00000125740 & $\begin{array}{l}\text { FBJ murine osteosarcoma viral } \\
\text { oncogene homolog B }\end{array}$ & 3.628551087 & 8.80E-05 & 0.032879001 \\
\hline$F Y B$ & ENSG00000082074 & FYN binding protein & -4.90648728 & 3.03E-05 & 0.016914087 \\
\hline GRPR & ENSG00000126010 & gastrin-releasing peptide receptor & -4.422707449 & 0.000144048 & 0.046095412 \\
\hline GSTT1 & ENSG00000184674 & glutathione S-transferase theta 1 & -9.843482395 & 6.94E-60 & $2.44 \mathrm{E}-55$ \\
\hline HIVEP3 & ENSG00000127124 & $\begin{array}{l}\text { human immunodeficiency virus type I } \\
\text { enhancer binding protein } 3\end{array}$ & -1.599082154 & $6.64 \mathrm{E}-05$ & 0.028486515 \\
\hline HOXC10 & ENSG00000180818 & homeobox $\mathrm{C} 10$ & -4.942455716 & 2.36E-05 & 0.014065434 \\
\hline HOXC-AS3 & ENSG00000251151 & HOXC cluster antisense RNA 3 & -5.14737488 & $6.01 \mathrm{E}-05$ & 0.026775571 \\
\hline IGF2 & ENSG00000167244 & $\begin{array}{l}\text { insulin-like growth factor } 2 \\
\text { (somatomedin A) }\end{array}$ & -4.063105964 & 3.83E-05 & 0.019780947 \\
\hline IGFBP1 & ENSG00000146678 & $\begin{array}{l}\text { insulin-like growth factor binding } \\
\text { protein } 1\end{array}$ & 2.926653654 & $1.58 \mathrm{E}-05$ & 0.010684692 \\
\hline
\end{tabular}




\begin{tabular}{|c|c|c|c|c|c|}
\hline IGFBP3 & ENSG00000146674 & $\begin{array}{l}\text { insulin-like growth factor binding } \\
\text { protein } 3\end{array}$ & 1.932377253 & $2.71 \mathrm{E}-06$ & 0.002574523 \\
\hline IL17RB & ENSG00000056736 & interleukin 17 receptor $B$ & 3.133016005 & 8.91E-05 & 0.032879001 \\
\hline IL20RA & ENSG00000016402 & interleukin 20 receptor, alpha & 3.104556086 & 3.12E-06 & 0.002749475 \\
\hline IQCE & ENSG00000106012 & IQ motif containing $E$ & -2.198435274 & $1.75 \mathrm{E}-43$ & 3.08E-39 \\
\hline IRF6 & ENSG00000117595 & interferon regulatory factor 6 & -3.931349479 & 7.74E-05 & 0.030946745 \\
\hline IRX1 & ENSG00000170549 & iroquois homeobox 1 & 1.496134127 & $2.70 \mathrm{E}-05$ & 0.015596484 \\
\hline$I R X 2$ & ENSG00000170561 & iroquois homeobox 2 & 1.161748374 & $4.51 \mathrm{E}-09$ & 1.77E-05 \\
\hline JAZF1-AS1 & ENSG00000234336 & JAZF1 antisense RNA 1 & -2.132735824 & 0.000107563 & 0.037119743 \\
\hline$K L K B 1$ & ENSG00000164344 & kallikrein B, plasma (Fletcher factor) 1 & 2.478417669 & 8.84E-05 & 0.032879001 \\
\hline LAMC1 & ENSG00000135862 & Iaminin, gamma 1 (formerly LAMB2) & -1.201150815 & $2.84 \mathrm{E}-07$ & 0.000453983 \\
\hline$L H X 9$ & ENSG00000143355 & LIM homeobox 9 & 4.298162126 & $1.45 \mathrm{E}-06$ & 0.001650048 \\
\hline LINC00537 & ENSG00000232815 & $\begin{array}{l}\text { long intergenic non-protein coding } \\
\text { RNA } 537\end{array}$ & -3.494641854 & 3.83E-05 & 0.019780947 \\
\hline LINC00578 & ENSG00000228221 & $\begin{array}{l}\text { long intergenic non-protein coding } \\
\text { RNA } 578\end{array}$ & -4.541164267 & 7.46E-09 & 2.39E-05 \\
\hline LINC01048 & ENSG00000230390 & $\begin{array}{l}\text { long intergenic non-protein coding } \\
\text { RNA } 1048\end{array}$ & 2.214309895 & 3.99E-05 & 0.019780947 \\
\hline LINC01117 & ENSG00000224577 & $\begin{array}{l}\text { long intergenic non-protein coding } \\
\text { RNA } 1117\end{array}$ & -2.17227763 & 2.17E-05 & 0.01316806 \\
\hline LRRC16A & ENSG00000079691 & leucine rich repeat containing $16 \mathrm{~A}$ & -1.784218256 & $2.43 \mathrm{E}-05$ & 0.014245821 \\
\hline MAP6 & ENSG00000171533 & microtubule-associated protein 6 & -1.79656788 & $1.30 \mathrm{E}-06$ & 0.001530605 \\
\hline MAP7D2 & ENSG00000184368 & MAP7 domain containing 2 & 2.359306267 & $2.66 \mathrm{E}-06$ & 0.002574523 \\
\hline MBP & ENSG00000197971 & myelin basic protein & -3.402601353 & 7.14E-05 & 0.029220727 \\
\hline MCCC1 & ENSG00000078070 & $\begin{array}{l}\text { methylcrotonoyl-CoA carboxylase } 1 \\
\text { (alpha) }\end{array}$ & -0.554719912 & 0.000128238 & 0.042584679 \\
\hline MECOM & ENSG00000085276 & MDS1 and EVI1 complex locus & -4.511721791 & 3.14E-09 & $1.58 \mathrm{E}-05$ \\
\hline METTL9 & ENSG00000197006 & methyltransferase like 9 & 0.906519962 & $3.86 \mathrm{E}-08$ & $9.70 \mathrm{E}-05$ \\
\hline MFGE8 & ENSG00000140545 & milk fat globule-EGF factor 8 protein & -2.694104724 & $7.44 \mathrm{E}-07$ & 0.000934855 \\
\hline MLLT3 & ENSG00000171843 & $\begin{array}{l}\text { myeloid/lymphoid or mixed-lineage } \\
\text { leukemia (trithorax homolog, } \\
\text { Drosophila); translocated to, } 3\end{array}$ & 0.969013225 & $2.10 \mathrm{E}-05$ & 0.01316806 \\
\hline MRVI1-AS1 & ENSG00000177112 & MRVI1 antisense RNA 1 & -3.884388307 & $1.04 \mathrm{E}-05$ & 0.007509783 \\
\hline MYO16 & ENSG00000041515 & myosin XVI & -1.962481605 & 0.000117031 & 0.039233207 \\
\hline NDP & ENSG00000124479 & Norrie disease (pseudoglioma) & 3.545635871 & $1.83 \mathrm{E}-05$ & 0.011727901 \\
\hline NR5A2 & ENSG00000116833 & $\begin{array}{l}\text { nuclear receptor subfamily } 5 \text {, group A, } \\
\text { member } 2\end{array}$ & -4.138425423 & 8.61E-06 & 0.006447287 \\
\hline NTRK3 & ENSG00000140538 & $\begin{array}{l}\text { neurotrophic tyrosine kinase, receptor, } \\
\text { type } 3\end{array}$ & 2.777060262 & 2.95E-05 & 0.016740854 \\
\hline PKIA & ENSG00000171033 & $\begin{array}{l}\text { protein kinase (cAMP-dependent, } \\
\text { catalytic) inhibitor alpha }\end{array}$ & -2.779337899 & 4.26E-05 & 0.020550766 \\
\hline$P N L D C 1$ & ENSG00000146453 & $\begin{array}{l}\text { poly(A)-specific ribonuclease (PARN)- } \\
\text { like domain containing } 1\end{array}$ & 2.95490495 & 2.27E-07 & 0.000399745 \\
\hline PNMA6C & ENSG00000235961 & $\begin{array}{l}\text { paraneoplastic Ma antigen family } \\
\text { member 6C }\end{array}$ & -4.033899706 & 0.000151809 & 0.047958078 \\
\hline POMZP3 & ENSG00000146707 & POM121 and ZP3 fusion & -1.717492119 & $3.12 \mathrm{E}-06$ & 0.002749475 \\
\hline POSTN & ENSG00000133110 & periostin, osteoblast specific factor & 2.83484857 & 2.14E-05 & 0.01316806 \\
\hline PRKAR1B & ENSG00000188191 & $\begin{array}{l}\text { protein kinase, cAMP-dependent, } \\
\text { regulatory, type I, beta }\end{array}$ & -1.732349926 & $1.78 \mathrm{E}-05$ & 0.011579369 \\
\hline PRLR & ENSG00000113494 & prolactin receptor & 3.300099077 & 0.000140582 & 0.045398939 \\
\hline PTK2B & ENSG00000120899 & protein tyrosine kinase 2 beta & -1.47679232 & $6.10 \mathrm{E}-05$ & 0.026827045 \\
\hline$R G C C$ & ENSG00000102760 & regulator of cell cycle & -3.93550099 & 7.06E-05 & 0.029220727 \\
\hline RHBDD3 & ENSG00000100263 & rhomboid domain containing 3 & -0.996433377 & $1.05 \mathrm{E}-05$ & 0.007509783 \\
\hline RSPO1 & ENSG00000169218 & R-spondin 1 & -4.313022549 & $5.98 \mathrm{E}-05$ & 0.026775571 \\
\hline S1PR1 & ENSG00000170989 & sphingosine-1-phosphate receptor 1 & -3.093512898 & $5.52 \mathrm{E}-06$ & 0.00452094 \\
\hline
\end{tabular}




\begin{tabular}{|c|c|c|c|c|c|}
\hline SCN1A & ENSG00000144285 & $\begin{array}{l}\text { sodium channel, voltage-gated, type I, } \\
\text { alpha subunit }\end{array}$ & -4.906276861 & 3.80E-06 & 0.003184547 \\
\hline SCN9A & ENSG00000169432 & $\begin{array}{l}\text { sodium channel, voltage-gated, type } \\
\text { IX, alpha subunit }\end{array}$ & -3.142530704 & $1.83 \mathrm{E}-06$ & 0.001898572 \\
\hline SDK1 & ENSG00000146555 & sidekick cell adhesion molecule 1 & 3.188897352 & 4.55E-05 & 0.021372366 \\
\hline SFRP1 & ENSG00000104332 & secreted frizzled-related protein 1 & -4.10661345 & $5.21 \mathrm{E}-07$ & 0.000732904 \\
\hline SHE & ENSG00000169291 & Src homology 2 domain containing $\mathrm{E}$ & 3.455259022 & 1.63E-06 & 0.001733572 \\
\hline$S L A$ & ENSG00000155926 & Src-like-adaptor & -2.701260288 & 3.99E-05 & 0.019780947 \\
\hline SLC16A11 & ENSG00000174326 & solute carrier family 16 , member 11 & -2.677392243 & 4.37E-05 & 0.020765473 \\
\hline SLC30A8 & ENSG00000164756 & $\begin{array}{l}\text { solute carrier family } 30 \text { (zinc } \\
\text { transporter), member } 8\end{array}$ & 6.104148946 & $1.35 \mathrm{E}-07$ & 0.000250798 \\
\hline SLC35D3 & ENSG00000182747 & solute carrier family 35 , member D3 & 9.179494017 & $2.46 \mathrm{E}-31$ & 2.89E-27 \\
\hline$S L C 4 A 3$ & ENSG00000114923 & $\begin{array}{l}\text { solute carrier family } 4 \text { (anion } \\
\text { exchanger), member } 3\end{array}$ & -2.366325383 & $7.28 \mathrm{E}-05$ & 0.029469479 \\
\hline SMOC1 & ENSG00000198732 & $\begin{array}{l}\text { SPARC related modular calcium } \\
\text { binding } 1\end{array}$ & -5.03813069 & 0.000104067 & 0.036268803 \\
\hline SPON2 & ENSG00000159674 & spondin 2 , extracellular matrix protein & -2.639592845 & 0.000112611 & 0.038484373 \\
\hline$S R D 5 A 2$ & ENSG00000049319 & \begin{tabular}{|l|} 
steroid-5-alpha-reductase, alpha \\
polypeptide 2 (3-oxo-5 alpha-steroid \\
delta 4-dehydrogenase alpha 2) \\
\end{tabular} & 4.448074003 & 8.97E-05 & 0.032879001 \\
\hline STAG3L2 & ENSG00000160828 & stromal antigen 3-like 2 (pseudogene) & -0.881204831 & $9.94 \mathrm{E}-05$ & 0.035716372 \\
\hline TCF21 & ENSG00000118526 & transcription factor 21 & -6.04609974 & $1.52 \mathrm{E}-06$ & 0.001676294 \\
\hline ULK4 & ENSG00000168038 & unc-51 like kinase 4 & 1.147447551 & 6.46E-09 & $2.28 \mathrm{E}-05$ \\
\hline WASF3 & ENSG00000132970 & WAS protein family, member 3 & 0.972046987 & 2.81E-06 & 0.002603617 \\
\hline$X K R 9$ & ENSG00000221947 & $\begin{array}{l}\text { XK, Kell blood group complex subunit- } \\
\text { related family, member } 9\end{array}$ & -1.989973823 & 0.000159916 & 0.049814665 \\
\hline ZBTB16 & ENSG00000109906 & $\begin{array}{l}\text { zinc finger and BTB domain containing } \\
16\end{array}$ & -4.844890791 & 7.83E-05 & 0.030946745 \\
\hline ZFPM2 & ENSG00000169946 & $\begin{array}{l}\text { zinc finger protein, FOG family } \\
\text { member } 2\end{array}$ & -3.60011069 & $5.25 \mathrm{E}-14$ & $4.62 \mathrm{E}-10$ \\
\hline ZNF804A & ENSG00000170396 & zinc finger protein $804 \mathrm{~A}$ & -5.483825868 & 4.34E-09 & 1.77E-05 \\
\hline
\end{tabular}


Supplementary Table S6. GO enrichment analysis. Show the distribution of GO terms exhibiting statistical significant differences of mis-regulated transcripts in A.IV-1 fibroblast using WebGestalt 2019 (Wang, Vasaikar, Shi, Greer, \& Zhang, 2017).

\begin{tabular}{|c|c|c|c|c|c|c|c|c|c|}
\hline GO terms & Description & $\begin{array}{c}\text { Gene } \\
\text { set size }\end{array}$ & Overlap & Expected Value & Enrichment Ratio & pValue & FDR & overlap_ld & user_Id \\
\hline GO:0009888 & tissue development & 1613 & 24 & 7.625171279802685 & 3.1474702822178497 & $1.528766712111107 \mathrm{e}-7$ & 0012670418509976855 & 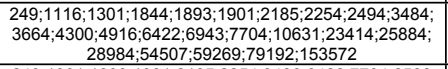 & 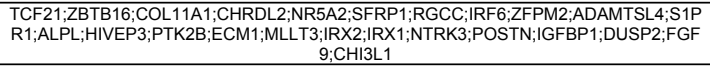 \\
\hline GO:0001503 & ossification & 339 & 11 & 1.6025623458481775 & 6.864007524261469 & $4.940246167706164 \mathrm{e}-7$ & 0020472380118974343 & $249 ; 1301 ; 1893 ; 1901 ; 2185 ; 2254 ; 33486 ; 6422 ; 7704 ; 2588$ & SMOC1;ZBTB16;COL11A1;CHRDL2;SFRP1;S1PR1;ALPL;PTK2B;ECM1;|GFBP3;FGF9 \\
\hline Go:0030038 & $\begin{array}{l}\text { Iractile actin filamen } \\
\text { assembly }\end{array}$ & 76 & 5 & 0.35927651411345574 & 13.916857360793287 & $2.9031950272262463 \mathrm{e}-5$ & 0.060154200964127824 & $1901 ; 2185 ; 6422 ; 28984 ; 55604$ & SFRP1;RGCC;S1PR1;CARMLL1;PTK2B \\
\hline G0:0043149 & stress fiber as & 76 & 5 & 0.35927651411345574 & 13.916857360793287 & $2.9031950272262463 \mathrm{e}-5$ & 0.060154200964127824 & $: 2185 ; 6422$ & SFRP1;RGCC;S1PR1;CARMIL1;PTK2B \\
\hline Go:0048584 & $\begin{array}{l}\text { positive regulation of response to } \\
\text { stimulus }\end{array}$ & 1768 & 21 & 8.35790627569197 & 2.512590989573087 & 4.138463705938289e-5 & 0.06101540527461857 & $\begin{array}{l}1116 ; 1123 ; 1893 ; 1901 ; 2070 ; 2185 ; 2254 ; 2533 ; 3486 ; 3381 \\
8 ; 4300 ; 4916 ; 5618 ; 642226653 ; 101417 ; 28984 ; 53832 ; 555 \\
40 ; 126669 ; 284654\end{array}$ & 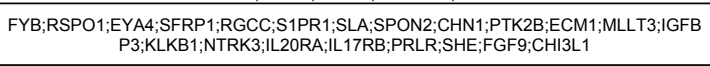 \\
\hline G0:0007379 & & $\frac{15}{160}$ & 3 & 0.07090 & 3768116 & $4.417138412737831 \mathrm{e}-5$ & 006101540527 & & \\
\hline G0:0030278 & & $\frac{169}{876}$ & $\begin{array}{ll}6 \\
13\end{array}$ & $\begin{array}{c}0.7989175116470265 \\
4.14113455741299\end{array}$ & $\begin{array}{l}7.510162078723952 \\
3.1392363179141025\end{array}$ & $\frac{1.4457514683041683 \mathrm{e}-4}{2.015277408380367 \mathrm{e}-4}$ & $\begin{array}{l}0.12546232977013955 \\
0.12546232977013955\end{array}$ & 6;;1301;1844; & 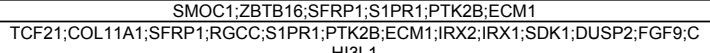 \\
\hline Go:0001501 & letal system development & 439 & 9 & 2.075294601260619 & 4.33673368327226 & $2.1232290176698143 \mathrm{e}-4$ & 0.12546232977013955 & $\begin{aligned} 84 ; 79192 ; 153532 ; 2221935 \\
249 ; 1116 ; 1301 ; 2254 ; 3226 ; 6422 ; 704 ; 10631 ; 25884 \\
\end{aligned}$ & $\begin{array}{c}\text { H13L1 } \\
\text { HOXC10;ZBTB16;COL11A1;CHRLL2;SFR1;ALPL;POSTN;FGF9;CHI3L1 }\end{array}$ \\
\hline GO:0072047 & $\begin{array}{l}\text { proximal/distal pattern formation } \\
\text { invulued in nephron development }\end{array}$ & 5 & 2 & 0.023636612770622088 & 84.61449275362318 & $2.182366244216638 \mathrm{e}-4$ & 0.12546232977013955 & $79192 ; 153572$ & 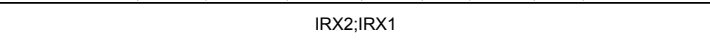 \\
\hline
\end{tabular}




\section{Supplementary Table S7. Summary of transcriptome mapping results.}

\begin{tabular}{|c|c|c|c|c|c|}
\hline Sample ID & \#total reads & \#aligned reads & $\begin{array}{c}\text { \% aligned } \\
\text { reads }\end{array}$ & $\begin{array}{c}\text { \#uniquely } \\
\text { mapped }\end{array}$ & $\begin{array}{c}\text { \% uniquely } \\
\text { aligned reads }\end{array}$ \\
\hline A.IV-1.1 & $306,265,974$ & $303,190,132$ & 99.00 & $293,189,972$ & 96.70 \\
\hline A.IV-1.2 & $344,782,588$ & $341,370,324$ & 99.01 & $330,182,914$ & 96.72 \\
\hline ARN1.1 & $338,129,498$ & $335,557,854$ & 99.24 & $325,618,112$ & 97.04 \\
\hline ARN1.2 & $301,339,526$ & $298,096,006$ & 98.92 & $289,521,446$ & 97.12 \\
\hline ARN2.1 & $311,917,536$ & $307,641,094$ & 98.63 & $296,837,798$ & 96.49 \\
\hline ARN2.2 & $336,751,354$ & $333,728,602$ & 99.10 & $322,903,810$ & 96.76 \\
\hline ARN3.1 & $403,718,870$ & $400,433,942$ & 99.19 & $383,740,470$ & 95.83 \\
\hline ARN3.2 & $299,879,030$ & $296,763,108$ & 98.96 & $286,203,188$ & 96.44 \\
\hline ARN4.1 & $318,588,494$ & $315,510,058$ & 99.03 & $304,438,002$ & 96.49 \\
\hline ARN4.2 & $320,107,070$ & $316,729,460$ & 98.94 & $304,559,776$ & 96.16 \\
\hline ARN5.1 & $363,584,984$ & $360,496,138$ & 99.15 & $349,081,194$ & 96.83 \\
\hline ARN6.1 & $324,984,406$ & $321,703,444$ & 98.99 & $311,631,678$ & 96.87 \\
\hline ARN6.2 & $364,763,088$ & $361,575,516$ & 99.13 & $349,853,468$ & 96.76 \\
\hline
\end{tabular}




\section{SUPPLEMENTARY REFERENCES}

Adzhubei, I. A., Schmidt, S., Peshkin, L., Ramensky, V. E., Gerasimova, A., Bork, P., . . Sunyaev, S. R. (2010). A method and server for predicting damaging missense mutations. Nature Methods, 7(4), 248-249. doi:10.1038/nmeth0410-248

Geoffroy, V., Pizot, C., Redin, C., Piton, A., Vasli, N., Stoetzel, C., .. Muller, J. (2015). VaRank: a simple and powerful tool for ranking genetic variants. PeerJ, 3, e796. doi:10.7717/peerj.796

Kim, D., Pertea, G., Trapnell, C., Pimentel, H., Kelley, R., \& Salzberg, S. L. (2013). TopHat2: accurate alignment of transcriptomes in the presence of insertions, deletions and gene fusions. Genome Biol, 14(4), R36. doi:10.1186/gb-2013-14-4-r36

Kumar, P., Henikoff, S., \& Ng, P. C. (2009). Predicting the effects of coding non-synonymous variants on protein function using the SIFT algorithm. Nature protocols, 4(7), 1073-1081. doi:10.1038/nprot.2009.86

Langmead, B., Trapnell, C., Pop, M., \& Salzberg, S. L. (2009). Ultrafast and memory-efficient alignment of short DNA sequences to the human genome. Genome Biol, 10(3), R25. doi:10.1186/gb-2009-10-3-r25

Love, M. I., Huber, W., \& Anders, S. (2014). Moderated estimation of fold change and dispersion for RNA-seq data with DESeq2. Genome Biol, 15(12), 550. doi:10.1186/s13059-014-0550-8

Nevers, Y., Prasad, M. K., Poidevin, L., Chennen, K., Allot, A., Kress, A., . . Lecompte, O. (2017). Insights into Ciliary Genes and Evolution from Multi-Level Phylogenetic Profiling. Mol Biol Evol, 34(8), 2016-2034. doi:10.1093/molbev/msx146

Reese, M. G., Eeckman, F. H., Kulp, D., \& Haussler, D. (1997). Improved splice site detection in Genie. J Comput Biol, 4(3), 311-323.

Shapiro, M. B., \& Senapathy, P. (1987). RNA splice junctions of different classes of eukaryotes: sequence statistics and functional implications in gene expression. Nucleic Acids Res, 15(17), 7155-7174.

Wang, J., Vasaikar, S., Shi, Z., Greer, M., \& Zhang, B. (2017). WebGestalt 2017: a more comprehensive, powerful, flexible and interactive gene set enrichment analysis toolkit. Nucleic Acids Research, 45(W1), W130-W137. doi:10.1093/nar/gkx356

Yeo, G., \& Burge, C. B. (2004). Maximum entropy modeling of short sequence motifs with applications to RNA splicing signals. J Comput Biol, 11(2-3), 377-394. doi:10.1089/1066527041410418 


\section{SUPPLEMENTARY INFORMATION}

\section{Sanger Sequencing}

Sanger sequencing was performed by way of PCR amplification with 50ng of genomic DNA template. The primers were designed with Primer 3 (http://frodo.wi.mit.edu/primer3). Bidirectional sequencing of the purified PCR products was performed by Eurofins Genomics Sequencing Facilities (https://www.eurofinsgenomics.eu/en/custom-dna-sequencing).

\section{Control screening}

IQCE exon 12 was tested in 93 control DNA of matched origin (Algerian) and 93 multi ethnic. High resolution melting (HRM) assay was performed in a $20 \mu$ reaction volume containing 50 $\mathrm{ng}$ of template DNA, $15 \mu \mathrm{l}$ Precision Melt Supermix (Bio-Rad) and $10 \mu \mathrm{mol}$ of each primer of the primer set. PCR was performed using a CFX96 (Bio-Rad) thermal cycler and consisted of predenaturation $\left(98^{\circ} \mathrm{C}\right.$ for $2 \mathrm{~min}$ ) followed by 35 cycles of amplification involving denaturation $\left(98^{\circ} \mathrm{C}\right.$ for $\left.5 \mathrm{~s}\right)$, annealing $\left(60^{\circ} \mathrm{C}\right.$ for $\left.10 \mathrm{~s}\right)$ and a melting curve involving denaturation $\left(95^{\circ} \mathrm{C}\right.$ for $30 \mathrm{~s})$, annealing $\left(70^{\circ} \mathrm{C}\right.$ for $\left.30 \mathrm{~s}\right)$ and a melt curve $\left(72-95^{\circ} \mathrm{C}\right.$ with an increment of $0.2^{\circ} \mathrm{C}$ per 10 s). Data generated were analyzed using Precision Melt Analysis software (Bio-Rad). In HRM analysis, differences in Tm and normalized curve shape were used together to discriminate even the most difficult-to-detect sequence variation.

\section{Bioinformatic pipeline RNA seq}

Image analysis and base calling were performed using RTA v.2.7.3 and blc2fastq v.2.17.1.14 (Illumina). Sequence reads were mapped onto hg19/GRCh37 assembly of human genome using Tophat 2.0.14 (Kim et al., 2013) and bowtie version 2-2.1.0 (Langmead, Trapnell, Pop, \& Salzberg, 2009), A summary of the mapping results is available in Supplementary Table 6. Gene expression was quantified from uniquely aligned reads using HTSeq-0.6.1 with gene annotations from Ensembl release 75 and intersection non-empty mode.

\section{Differential expression RNA-seq}

In order to identify variation in mRNA expression due to the absence of IQCE in the patient cells, comparisons of read counts were performed using $R$ 3.2.5 with the statistical method proposed by Anders and Huber implemented in the DESeq2 v.1.10.1 Bioconductor package 
(Love, et al., 2014). The generated data were explored and visualized to assess and check data quality and to eventually remove bad quality data. Especially, PCA was built after stabilizing variance using the regularized log transformation method or RSeqQC was used to do some quality controls such as gene body coverage. Only genes with | log2 fold-change | > 0,5 were considered, as we expected to identify a large amount of change between transcriptomic results from one patient compared to other individuals (indirect consequences of the pathogenic mutation or unrelated events due to variability between humans). To avoid as much as possible false positive variants, we filtered out all genes with a Benjamini and Hochberg adjusted p-value above 0.05 . 
A

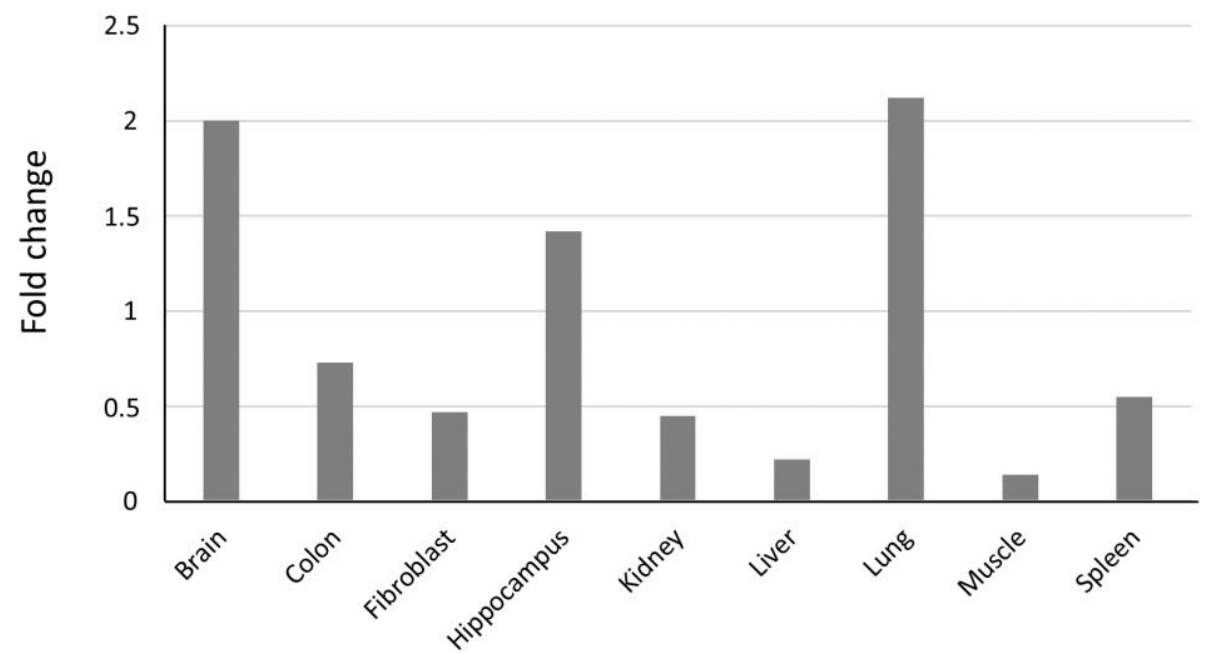

B

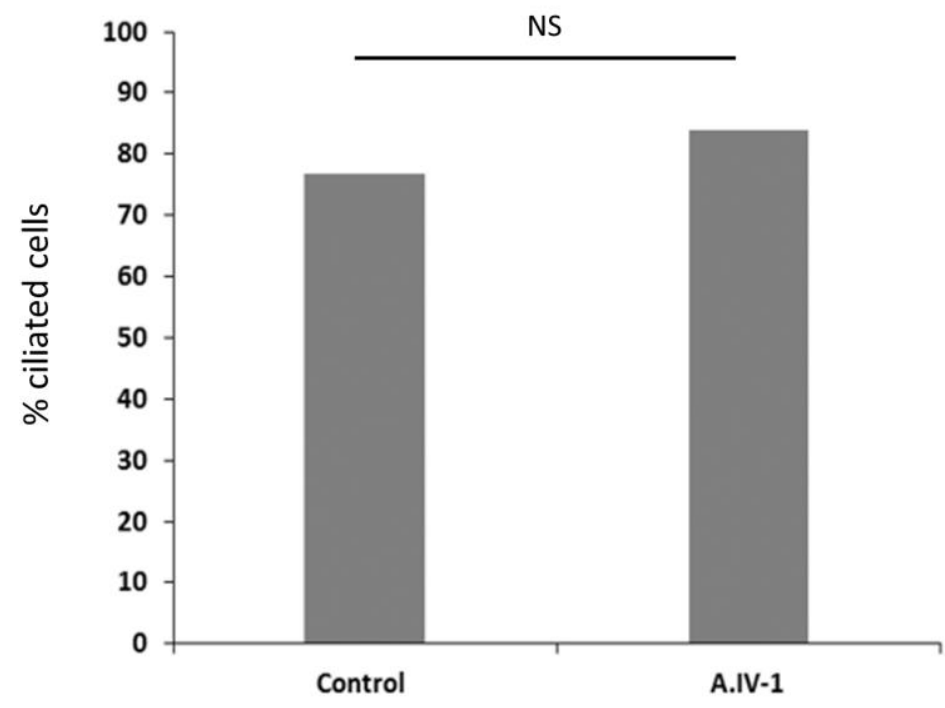

Supplementary Figure S1. A. Highest mRNA expression of IQCE in adult human brain/hippocampus and lung. B. Count of ciliated fibroblasts between A.IV-1 and control ( 100 cells per condition). Percentages are shown in histogram, N.S.: non-significant, Fisher exact test $0.224, P<0.05)$. 


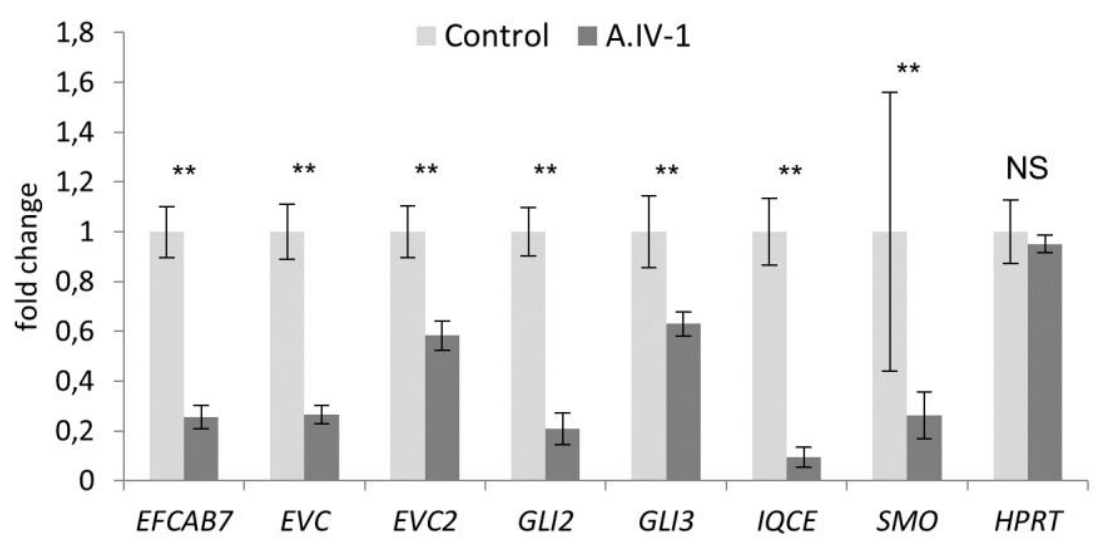

Supplementary Figure S2. Expression levels of EVC-zone and GLI genes upon ciliogenesis and Hh activation. Patient's (A.IV-1) and control cells upon treatment (-FCS + SAG $100 n M$ ) were compared ( $n=4$ controls, $n=6$ patient). Statistical significance has been performed using the non-parametric Mann-Whitney test, $p=0.0095\left(^{* \star}\right)$, NS : non-significant. 
A

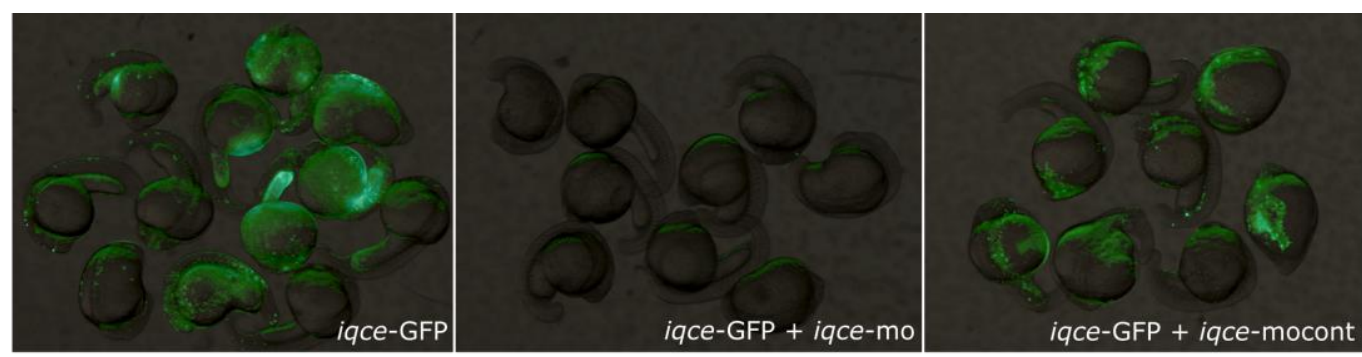

B
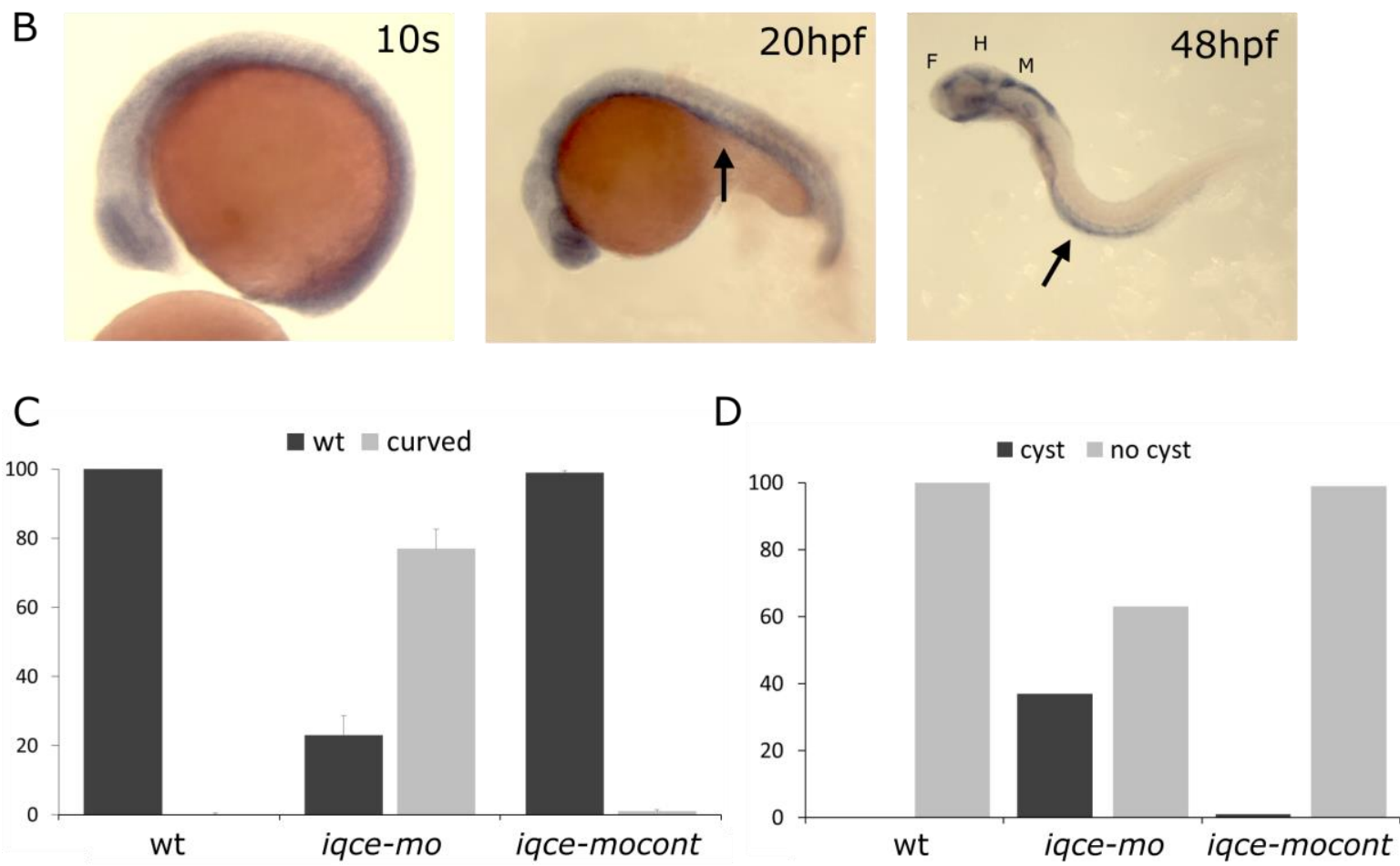

$\mathrm{E}$

$\mathrm{F}$
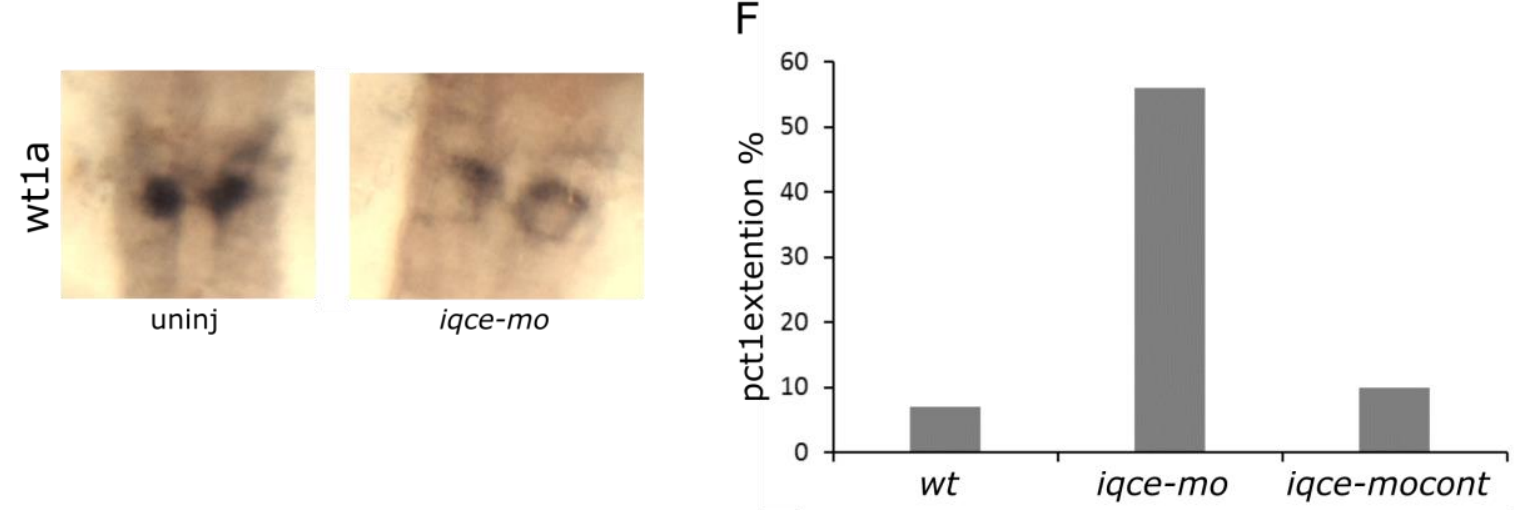

Supplementary Figure S3. Efficacy of iqce morpholinos. A. Morpholinos directed against the start codon of iqce blocks the expression of a construct containing iqce cDNA in frame with gfp (central panel). In contrast, 5 mismatch morpholinos did not reduce expression of the chimeric protein (left panel) compared to iqce-gfp alone (right panel). $24 \mathrm{hpf}$. B. in situ hybridization showing iqce expression pattern in 10 somites, 20 and $48 \mathrm{hpf}$ embryos. Arrow indicates the pronephric duct. $\mathrm{F}$ : forebrain ventricule; $\mathrm{H}$ : hindbrain ventricule; $\mathrm{M}$ : midbrain ventricule. C. Chart showing the percentage of curved embryos in uninjected $(n=200)$, iqce- 
mo ( $\mathrm{n}=120)$; and iqce-mocont $(\mathrm{n}=221)$ injected embryos. $48 \mathrm{hpf}$, data includes $\pm S D$ and statistical significance was determined using the Student's t-test, all comparisons were significant with a $p$-value<0.05. D. Chart showing the percentage of cysts in uninjected $(\mathrm{n}=101)$, iqce-mo $(\mathrm{n}=70)$ and iqce-mocont $(\mathrm{n}=90)$ injected embryos. Statistical significance was determined using the Student's t-test, all comparisons were significant with a p-value<0.05. E. wt1a in situ hybridization showing expression within glomeruli in non-injected embryos and at the periphery of the cyst in morphants (48 hpf). F. Chart showing the percentage of embryos with ptc1 extension in 10 somites embryos $(n=191)$. 
Supplementary Table S1. Summary of the whole exome sequencing results. SNV: Single nucleotide variation, indel: gain or loss of up to 50 nucleotides at a single locus, SV: Structural Variation. Exclusion of SV with a DGV frequency $>1 \%$ is done only with studies of more than 1000 individuals

\begin{tabular}{|c|c|c|c|c|c|c|c|c|c|c|c|c|}
\hline & \multicolumn{3}{|c|}{ A.IV-1 } & \multicolumn{3}{|c|}{ B.II-2 } & \multicolumn{3}{|c|}{ B.II-3 } & \multicolumn{3}{|c|}{ C.IV-4 } \\
\hline & SNV & indel & sv & SNV & indel & sv & SNV & Indel & sv & SNV & Indel & sv \\
\hline Total number of variants & 83129 & 11664 & 31 & 81972 & 11319 & 17 & 82116 & 11195 & 16 & 78906 & 11727 & 31 \\
\hline $\begin{array}{l}\text { After exclusion of variants with an allele frequency }>1 \% \\
\text { (gnomAD, } 1000 \mathrm{G} \text {, internal exome database, DGV) }\end{array}$ & 9771 & 1125 & 16 & 7069 & 993 & 6 & 6856 & 943 & 9 & 5323 & 982 & 17 \\
\hline $\begin{array}{l}\text { After exclusion of SNV/indel in 5'UTR, 3'UTR, downstream, } \\
\text { upstream, intron and synonymous locations without local splice } \\
\text { effect prediction }\end{array}$ & 788 & 76 & 1 & 553 & 59 & 1 & 560 & 65 & 1 & 753 & 113 & l \\
\hline $\begin{array}{l}\text { After exclusion of missense with no deleterious effect } \\
\text { according to SIFT, PPH2 or PhastCons }\end{array}$ & 669 & 76 & l & 458 & 59 & l & 465 & 65 & 1 & 641 & 113 & l \\
\hline $\begin{array}{l}\text { After exclusion of variants not in a ciliary genes list (Nevers et } \\
\text { al., 2017) and not in non-syndromic polydactyly known genes }\end{array}$ & 52 & 6 & 1 & 31 & 2 & 2 & 40 & 4 & 2 & 78 & 15 & 1 \\
\hline $\begin{array}{l}\text { After selection of variants consistent with recessive } \\
\text { transmission (compound heterozygous, homozygous variants) }\end{array}$ & $\begin{array}{r}7 \text { hom. } \\
\text { SHA } \\
3 \text { comp. h }\end{array}$ & $\begin{array}{l}\text { JAAF1, IC } \\
\text { TULP1, } \\
\text { ARMC3, }\end{array}$ & $\begin{array}{l}\text { IADD, } \\
\text { HC2 } \\
11, M G A\end{array}$ & & $\begin{array}{l}0 \text { hom } \\
1 \mathrm{com}\end{array}$ & $\begin{array}{l}\text { ozyg } \\
\text { ip. he }\end{array}$ & $\begin{array}{l}\text { ous vari } \\
\text { et. In } I Q C\end{array}$ & & & $\begin{array}{r}3 \text { hom.: } \\
11 \mathrm{com} \\
\text { DNAH7 } \\
\text { LRRCC1, } \\
\text { SMA }\end{array}$ & $\begin{array}{l}\text { T1, AGTP } \\
\text { et: CTDS } \\
\text { NLL1, EF } \\
\text { C46, MR } \\
\text { 5, TMEM }\end{array}$ & $\begin{array}{l}\text {, KDM4C } \\
\text { DDX1, } \\
\text {, IFT20, } \\
\text {, PRKDC, } \\
\text { TPTE }\end{array}$ \\
\hline
\end{tabular}


Supplementary Table S2. Homozygous regions identified in individual A.IV-1.

\begin{tabular}{|c|c|c|c|c|c|c|c|c|}
\hline Chr & SNP1 & SNP2 & POS1 & POS2 & MB & \#SNP & DENSITY & \\
\hline 1 & rs4147830 & rs11431894 & 94544276 & 100680594 & 6.14 & 33 & 185.949 & \\
\hline 1 & rs4915053 & rs3761934 & 108113526 & 114189067 & 6.08 & 136 & 44.673 & \\
\hline 1 & 1_148741720_T_C & rs11428742 & 148741720 & 150550020 & 1.81 & 22 & 82.196 & \\
\hline 1 & rs 4636 & rs10927011 & 234744413 & 243579112 & 8.83 & 125 & 70.678 & \\
\hline 2 & rs7577088 & rs3764906 & 39233500 & 42588195 & 3.35 & 20 & 167.735 & \\
\hline 2 & rs522893 & 2_188250301_T_A & 169690729 & 188250301 & 18.56 & 224 & 82.855 & \\
\hline 2 & rs2304704 & rs12477076 & 190430177 & 191848312 & 1.42 & 23 & 61.658 & \\
\hline 3 & rs901854 & rs113574694 & 4856234 & 10089550 & 5.23 & 58 & 90.230 & \\
\hline 3 & rs9814557 & rs2246945 & 135720540 & 137843476 & 2.12 & 21 & 101.092 & \\
\hline 4 & rs13146318 & rs1051447 & 41615690 & 49063872 & 7.45 & 68 & 109.532 & \\
\hline 4 & rs4864727 & rs12621 & 54011424 & 57897570 & 3.89 & 61 & 63.707 & \\
\hline 4 & rs10434219 & rs344122 & 62598689 & 77637502 & 15.04 & 175 & 85.936 & \\
\hline 6 & rs2076506 & 6_32485853_C_T & 22570064 & 32485853 & 9.92 & 646 & 15.350 & \\
\hline 6 & rs1059547 & rs12189627 & 32557529 & 41059458 & 8.50 & 309 & 27.514 & TULP1 \\
\hline 7 & rs4916935 & rs798479 & 197267 & 2691201 & 2.49 & 41 & 60.828 & $I Q C E$ \\
\hline 7 & rs7794563 & rs80024169 & 5347295 & 14775824 & 9.43 & 78 & 120.879 & \\
\hline 7 & rs4718101 & rs6460315 & 64152353 & 66289040 & 2.14 & 38 & 56.229 & \\
\hline 8 & rs3989699 & rs13271468 & 7218621 & 12236202 & 5.02 & 132 & 38.012 & \\
\hline 8 & 8_12273335_G_A & rs66480340 & 12273335 & 24211885 & 11.94 & 202 & 59.102 & \\
\hline 8 & rs10086200 & rs59331088 & 61193588 & 74005131 & 12.81 & 72 & 177.938 & \\
\hline 8 & rs11777189 & rs12545587 & 99057150 & 136560952 & 37.50 & 204 & 183.842 & \\
\hline 9 & rs4977555 & rs11515 & 19550308 & 21968199 & 2.42 & 30 & 80.596 & \\
\hline 9 & rs7863560 & rs3217100 & 119495697 & 123734288 & 4.24 & 30 & 141.286 & \\
\hline 11 & 11_32452000_GG_- & rs2076623 & 32452000 & 33564123 & 1.11 & 25 & 44.485 & \\
\hline 11 & rs12274095 & rs7101792 & 69063767 & 71249386 & 2.19 & 28 & 78.058 & \\
\hline 11 & rs3740912 & rs4937391 & 125891269 & 128786294 & 2.90 & 32 & 90.470 & \\
\hline 12 & rs11054683 & rs1600 & 12247616 & 14656768 & 2.41 & 31 & 77.715 & \\
\hline 12 & rs3088008 & rs3210837 & 49724955 & 51685831 & 1.96 & 55 & 35.652 & \\
\hline 12 & rs74651927 & rs2242497 & 52695754 & 56992630 & 4.30 & 185 & 23.226 & \\
\hline 12 & rs1663564 & rs7488309 & 105546172 & 118520170 & 12.97 & 152 & 85.355 & \\
\hline 12 & rs1794956 & rs11059841 & 124848137 & 129190021 & 4.34 & 37 & 117.348 & \\
\hline 14 & rs34800262 & rs2249922 & 51370852 & 52906081 & 1.54 & 34 & 45.154 & \\
\hline 14 & rs2297113 & rs863091 & 58036494 & 59112475 & 1.08 & 23 & 46.782 & \\
\hline 14 & rs35533709 & rs2057482 & 60433392 & 62213848 & 1.78 & 30 & 59.349 & \\
\hline 15 & rs4923929 & rs555001 & 42439376 & 43545728 & 1.11 & 37 & 29.901 & \\
\hline 15 & rs11351249 & rs2623989 & 72105929 & 73545732 & 1.44 & 23 & 62.600 & \\
\hline 15 & rs752270 & rs4777755 & 87099537 & 93510603 & 6.41 & 171 & 37.492 & \\
\hline 16 & rs6564764 & rs931713 & 80581631 & 85023855 & 4.44 & 121 & 36.713 & \\
\hline 17 & rs8071836 & rs67749219 & 47238028 & 49711042 & 2.47 & 40 & 61.825 & \\
\hline
\end{tabular}


Supplementary Table S3. List and characteristics of primers used in this study

\begin{tabular}{|c|c|c|c|c|c|}
\hline Application & Gene & Exon & Forward (5'-3') & Reverse (5'-3') & $\begin{array}{l}\text { Size } \\
\text { (bp) }\end{array}$ \\
\hline \multirow[t]{4}{*}{ DNA } & IQCE & 12 & CTCTGAGCTCACAACCAACAG & GTTGCCCAAGGGTTCACA & 399 \\
\hline & & 16 & TGGTTTCTGGTGTTGTCAGG & TTTTCAGTGGCTGGTCAGAA & 382 \\
\hline & ATP6V1B1 & 3 & AGCCGTGGGAAGTAGGTGTT & ATGTCCCATTGACCCCACT & 385 \\
\hline & TULP1 & $12 / 13$ & GGGATGTAGGATCCCCTCA & CATGCCAGGAATGATGACG & 481 \\
\hline \multirow[t]{17}{*}{ cDNA } & $I Q C E$ & $3-4 / 5$ & ACATCGCCAAAGTCACCTTATC & AGGGCCTGGGTCAGACTT & 158 \\
\hline & GSTT1 & $2-3 / 3$ & ACCTTGACGGAGAGTGTGG & GCAGCTTCTCCGCAGAGT & 144 \\
\hline & SLC30A8 & $9-10 / 10$ & CCATCTTACTCATGGAAGGTGTG & GCTGTAGCAACATGAGCTGAGA & 154 \\
\hline & RSPO1 & $4 / 5$ & TGGAGAGGAACGACATCCG & CCTTACACTTGGTGCAGAAGTTA & 156 \\
\hline & FGF9 & $2 / 3$ & GGCCTGGTCAGCATTCGAG & GTATCGCCTTCCAGTGTCCAC & 180 \\
\hline & SFRP1 & $1 / 2$ & GCTTCTACTGGCCCGAGATG & TGGCCTCAGATTTCAACTCGT & 180 \\
\hline & MECOM & $4 / 5$ & AAACTCGAAAGCGAGAATGATCT & TGGTGGCGAATTAAATTGGACTT & 185 \\
\hline & $A L P L$ & $6-7 / 7$ & AACATCAGGGACATTGACGTG & GTATCTCGGTTTGAAGCTCTTCC & 159 \\
\hline & IGF2 & $2-3 / 3-4$ & TTCTACTTCAGCAGGCCCG & GAAGTTGTCCGGAAGCACG & 168 \\
\hline & $G A P D H$ & $4-5 / 6$ & GGAGCGAGATCCCTCCAAAAT & GGCTGTTGTCATACTTCTCATGG & 197 \\
\hline & HPRT1 & $1-2 / 2-3$ & CCTGGCGTCGTGATTAGTGAT & AGACGTTCAGTCCTGTCCATAA & 131 \\
\hline & $E F C A B 7$ & $6 / 6-7$ & GTCAGCAACCAGGAAGTTCA & CATGTGTTGCCAGTCCTTTATT & 115 \\
\hline & EVC2 & $6-7 / 8$ & CTCGTCACGGAACAGAACAC & CAGGTCAGCACAAGGGAGAG & 135 \\
\hline & $E V C$ & $2 / 4$ & TCGAAGGACAAGGAAGCTGT & TTTCATGCAGAGACGGGTTG & 148 \\
\hline & GLI2 & $2 / 3$ & AAGCAAGAAGCCAAAAGTGG & TGGTACCTTCCTTCCTGGTG & 188 \\
\hline & GLI3 & $2 / 3$ & GGCATTTTTTGGTCGAAGAGA & GGACATTCTGTGGCTGCATA & 238 \\
\hline & SMO & $2 / 3$ & TGCCCAAGTGTGAGAATGAC & TACCAGCTCTTGGGGTTGTC & 229 \\
\hline
\end{tabular}


Supplementary Table S4. Comparison of haplotypes of individuals A.IV-1 and B.II-2.

\begin{tabular}{|c|c|c|c|c|c|c|c|}
\hline ID & Gene & Chr & Position & Allele_1_A.IV-1 & Allele_2_A.IV-1 & $\begin{array}{l}\text { Allele_1_II- } \\
\text { 2_Family2 }\end{array}$ & $\begin{array}{l}\text { Allele_1_II- } \\
\text { 2_Family2 }\end{array}$ \\
\hline 7_2255974_C_T & MAD1L1 & 7 & 2255974 & $\mathrm{C}$ & C & C & $\mathrm{T}$ \\
\hline 7_2256017_C_A & MAD1L1 & 7 & 2256017 & $\mathrm{C}$ & C & $\mathrm{C}$ & $A$ \\
\hline 7_2257612_G_A & MAD1L1 & 7 & 2257612 & A & $A$ & $A$ & $\mathrm{G}$ \\
\hline 7_2259134_C_T & MAD1L1 & 7 & 2259134 & C & C & C & $\mathrm{T}$ \\
\hline 7_2260715_G_T & MAD1L1 & 7 & 2260715 & $\mathrm{~T}$ & $\mathrm{~T}$ & $\mathrm{~T}$ & $\mathrm{~T}$ \\
\hline 7_2269552_G_T & MAD1L1 & 7 & 2269552 & $\mathrm{~T}$ & $\mathrm{~T}$ & $\mathrm{~T}$ & $\mathrm{G}$ \\
\hline 7_2278663_T_A & FTSJ2 & 7 & 2278663 & $\mathrm{~T}$ & $\mathrm{~T}$ & $A$ & $A$ \\
\hline 7_2279482_A_C & FTSJ2 & 7 & 2279482 & A & $A$ & C & C \\
\hline 7_2279851_T_C & FTSJ2 & 7 & 2279851 & $\mathrm{~T}$ & $\mathrm{~T}$ & C & C \\
\hline 7_2281754_C_T & FTSJ2 & 7 & 2281754 & C & C & C & $\mathrm{T}$ \\
\hline 7_2290328_C_T & SNX8 & 7 & 2290328 & C & C & C & $\mathrm{T}$ \\
\hline 7_2290336_T_G & SNX8 & 7 & 2290336 & $\mathrm{~T}$ & $\mathrm{~T}$ & $\mathrm{~T}$ & $G$ \\
\hline 7_2290372_C_T & SNX8 & 7 & 2290372 & C & C & $\mathrm{T}$ & $\mathrm{T}$ \\
\hline 7_2290522_C_T & SNX8 & 7 & 2290522 & $\mathrm{~T}$ & $\mathrm{~T}$ & C & C \\
\hline 7_2292881_-_TG & SNX8 & 7 & 2292881 & - & - & $\mathrm{TG}$ & $\mathrm{TG}$ \\
\hline 7_2296493_A_G & SNX8 & 7 & 2296493 & $\mathrm{G}$ & $G$ & $\mathrm{G}$ & $\mathrm{G}$ \\
\hline 7_2297006_A_G & SNX8 & 7 & 2297006 & A & A & $\mathrm{G}$ & $\mathrm{G}$ \\
\hline 7_2303000_G_A & SNX8 & 7 & 2303000 & $A$ & $A$ & $\mathrm{G}$ & $\mathrm{G}$ \\
\hline 7_2303109_G_A & SNX8 & 7 & 2303109 & $\mathrm{G}$ & G & G & A \\
\hline 7_2303986_T_C & SNX8 & 7 & 2303986 & C & C & C & C \\
\hline 7_2304191_A_C & SNX8 & 7 & 2304191 & $A$ & A & $\mathrm{C}$ & C \\
\hline 7_2309159_-_A & SNX8 & 7 & 2309159 & - & - & - & A \\
\hline 7_2309174_G_A & SNX8 & 7 & 2309174 & $\mathrm{G}$ & G & $\mathrm{G}$ & A \\
\hline 7_2309185_G_A & SNX8 & 7 & 2309185 & $\mathrm{G}$ & G & $\mathrm{G}$ & A \\
\hline 7_2309209_C_T & SNX8 & 7 & 2309209 & C & C & C & $\mathrm{T}$ \\
\hline 7_2314652_G_A & SNX8 & 7 & 2314652 & $G$ & $\mathrm{G}$ & $\mathrm{G}$ & A \\
\hline 7_2315008_G_C & SNX8 & 7 & 2315008 & $\mathrm{G}$ & G & C & C \\
\hline 7_2318254_ACTC_- & SNX8 & 7 & 2318254 & - & - & ACTC & ACTC \\
\hline 7_2318268_C_T & SNX8 & 7 & 2318268 & $\mathrm{~T}$ & $\mathrm{~T}$ & C & C \\
\hline 7_2318463_C_T & SNX8 & 7 & 2318463 & C & $\mathrm{T}$ & C & C \\
\hline 7_2318472_C_A & SNX8 & 7 & 2318472 & C & A & C & C \\
\hline 7_2349470_T_C & SNX8 & 7 & 2349470 & $\mathrm{~T}$ & $\mathrm{~T}$ & $\mathrm{~T}$ & C \\
\hline 7_2394991_C_T & EIF3B & 7 & 2394991 & C & C & C & $\mathrm{T}$ \\
\hline 7_2400306_T_C & EIF3B & 7 & 2400306 & C & C & C & $\mathrm{T}$ \\
\hline 7_2405885_C_A & EIF3B & 7 & 2405885 & $A$ & $A$ & $A$ & C \\
\hline 7_2409035_C_T & EIF3B & 7 & 2409035 & $\mathrm{C}$ & C & C & $\mathrm{T}$ \\
\hline 7_2414142_A_G & EIF3B & 7 & 2414142 & $\mathrm{G}$ & G & $\mathrm{G}$ & A \\
\hline 7_2414365_A_G & EIF3B & 7 & 2414365 & A & A & A & G \\
\hline 7_2416822_A_G & EIF3B & 7 & 2416822 & $A$ & $A$ & $A$ & $\mathrm{G}$ \\
\hline 7_2418645_A_G & EIF3B & 7 & 2418645 & A & A & A & G \\
\hline 7_2433729_C_A & NA & 7 & 2433729 & C & C & C & A \\
\hline 7_2434522_G_A & NA & 7 & 2434522 & $G$ & G & A & A \\
\hline 7_2434596_TT_- & NA & 7 & 2434596 & $\mathrm{TT}$ & $\mathrm{TT}$ & $\mathrm{TT}$ & - \\
\hline 7_2434597_T_- & NA & 7 & 2434597 & $\mathrm{~T}$ & $\mathrm{~T}$ & $\mathrm{~T}$ & - \\
\hline
\end{tabular}




\begin{tabular}{|c|c|c|c|c|c|c|c|}
\hline 7_2472429_C_A & CHST12 & 7 & 2472429 & C & $\mathrm{C}$ & C & $A$ \\
\hline 7_2472455_A_T & CHST12 & 7 & 2472455 & $A$ & $A$ & $A$ & $\mathrm{~T}$ \\
\hline 7_2489311_C_G & NA & 7 & 2489311 & C & C & C & $G$ \\
\hline $\begin{array}{l}\text { 7_2489336_GTGTGT } \\
\text { GCACCT__- }\end{array}$ & NA & 7 & 2489336 & GTGTGTGCACCT & GTGTGTGCACCT & GTGTGTGCACCT & - \\
\hline 7_2489382_A_G & NA & 7 & 2489382 & $A$ & $A$ & A & $\mathrm{G}$ \\
\hline 7_2515382_-_C & NA & 7 & 2515382 & - & - & C & C \\
\hline 7_2517473_C_T & NA & 7 & 2517473 & $\mathrm{C}$ & C & C & $\mathrm{T}$ \\
\hline 7_2552986_A_C & $L F N G$ & 7 & 2552986 & $A$ & $A$ & $A$ & $\mathrm{C}$ \\
\hline 7_2553030_T_C & $L F N G$ & 7 & 2553030 & $\mathrm{~T}$ & $\mathrm{~T}$ & $\mathrm{~T}$ & C \\
\hline 7_2557266_T_C & $L F N G$ & 7 & 2557266 & $\mathrm{~T}$ & $\mathrm{~T}$ & C & C \\
\hline 7_2557377_T_C & $L F N G$ & 7 & 2557377 & $\mathrm{~T}$ & $\mathrm{~T}$ & $\mathrm{~T}$ & C \\
\hline 7_2565268_C_A & LFNG/MIR4648 & 7 & 2565268 & C & C & $A$ & $A$ \\
\hline 7_2566433_G_A & LFNG/MIR4648 & 7 & 2566433 & $G$ & $G$ & $G$ & $A$ \\
\hline 7_2577691_C_G & BRAT1 & 7 & 2577691 & C & C & C & $G$ \\
\hline 7_2577781_T_C & BRAT1 & 7 & 2577781 & $\mathrm{~T}$ & $\mathrm{~T}$ & $\mathrm{~T}$ & C \\
\hline 7_2578237_T_C & BRAT1 & 7 & 2578237 & $\mathrm{~T}$ & $\mathrm{~T}$ & $\mathrm{~T}$ & C \\
\hline 7_2578238_C_T & BRAT1 & 7 & 2578238 & C & C & C & $\mathrm{T}$ \\
\hline 7_2578434_G_T & BRAT1 & 7 & 2578434 & $\mathrm{G}$ & $G$ & $G$ & $\mathrm{~T}$ \\
\hline 7_2578455_G_A & BRAT1 & 7 & 2578455 & $\mathrm{G}$ & $\mathrm{G}$ & $\mathrm{G}$ & $A$ \\
\hline 7_2580914_C_T & BRAT1 & 7 & 2580914 & $\mathrm{C}$ & C & $\mathrm{C}$ & $\mathrm{T}$ \\
\hline 7_2581994_T_C & BRAT1 & 7 & 2581994 & $\mathrm{~T}$ & $\mathrm{~T}$ & $\mathrm{~T}$ & C \\
\hline $\begin{array}{l}\text { 7_2582154_- } \\
\text { CCCCAGC̄CTCCCG } \\
\text { GGTGT }\end{array}$ & BRAT1 & 7 & 2582154 & $\begin{array}{l}\text { CCCCAGCCTCC } \\
\text { CGGGTGT }\end{array}$ & $\begin{array}{l}\text { CCCCAGCCTCC } \\
\text { CGGGTGT }\end{array}$ & - & - \\
\hline 7_2582233_A_G & BRAT1 & 7 & 2582233 & $A$ & $A$ & $A$ & $\mathrm{G}$ \\
\hline 7_2582381_T_A & BRAT1 & 7 & 2582381 & $\mathrm{~T}$ & $\mathrm{~T}$ & $\mathrm{~T}$ & $A$ \\
\hline 7_2582584_G_A & BRAT1 & 7 & 2582584 & $G$ & $\mathrm{G}$ & $G$ & $A$ \\
\hline 7_2583165_G_A & BRAT1 & 7 & 2583165 & $G$ & $G$ & $\mathrm{G}$ & A \\
\hline 7_2583328_C_T & BRAT1 & 7 & 2583328 & $\mathrm{C}$ & $\mathrm{C}$ & $\mathrm{C}$ & $\mathrm{T}$ \\
\hline 7_2584523_T_C & BRAT1 & 7 & 2584523 & $\mathrm{~T}$ & $\mathrm{~T}$ & $\mathrm{~T}$ & $\mathrm{C}$ \\
\hline 7_2587122_T_C & BRAT1 & 7 & 2587122 & $\mathrm{~T}$ & $\mathrm{~T}$ & $\mathrm{~T}$ & C \\
\hline 7_2598757_T_C & $I Q C E$ & 7 & 2598757 & $\mathrm{~T}$ & $\mathrm{~T}$ & $\mathrm{~T}$ & C \\
\hline 7_2604768_GTGT_- & $I Q C E$ & 7 & 2604768 & GTGT & GTGT & GTGT & - \\
\hline 7_2604769_T_* & IQCE & 7 & 2604769 & $\mathrm{~T}$ & $\mathrm{~T}$ & $\mathrm{~T}$ & * \\
\hline 7_2604816_C_T & IQCE & 7 & 2604816 & $\mathrm{C}$ & C & C & $\mathrm{T}$ \\
\hline 7_2611878_T_C & $I Q C E$ & 7 & 2611878 & $\mathrm{~T}$ & $\mathrm{~T}$ & $\mathrm{~T}$ & C \\
\hline 7_2612225_G_A & $I Q C E$ & 7 & 2612225 & A & $A$ & $\mathrm{G}$ & $\mathrm{G}$ \\
\hline 7_2613042_G_C & IQCE & 7 & 2613042 & $G$ & G & G & C \\
\hline 7_2618070_-_A & $I Q C E$ & 7 & 2618070 & - & - & - & $A$ \\
\hline 7_2619341_GT_- & $I Q C E$ & 7 & 2619341 & GT & GT & GT & - \\
\hline 7_2619363_G_C & $I Q C E$ & 7 & 2619363 & $\mathrm{G}$ & $\mathrm{G}$ & $\mathrm{G}$ & C \\
\hline 7_2623351_G_A & IQCE & 7 & 2623351 & $G$ & $G$ & $\mathrm{G}$ & A \\
\hline 7_2623801_G_T & IQCE & 7 & 2623801 & $G$ & $G$ & $\mathrm{G}$ & $\mathrm{T}$ \\
\hline $\begin{array}{l}\text { 7_2625907_TGTCCC } \\
\text { GGAG_- }\end{array}$ & $I Q C E$ & 7 & 2625907 & - & - & - & TGTCCCGGAG \\
\hline $\begin{array}{l}\text { 7_2626039_CAGGGA } \\
\text { ATGG_- }\end{array}$ & $I Q C E$ & 7 & 2626039 & CAGGGAATGG & CAGGGAATGG & CAGGGAATGG & - \\
\hline 7_2627310_T_A & $I Q C E$ & 7 & 2627310 & $\mathrm{~T}$ & $\mathrm{~T}$ & $\mathrm{~T}$ & A \\
\hline 7_2627579_AG_- & $I Q C E$ & 7 & 2627579 & $A G$ & $A G$ & - & - \\
\hline 7_2632477_A_G & IQCE & 7 & 2632477 & A & $A$ & $A$ & $G$ \\
\hline
\end{tabular}




\begin{tabular}{|c|c|c|c|c|c|c|}
\hline 7_2634518_AGAG_- & $I Q C E$ & 7 & 2634518 & AGAG & AGAG & AGAG \\
\hline 7_2636689_C_T & $I Q C E$ & 7 & 2636689 & $\mathrm{C}$ & C & C \\
\hline 7_2638347_C_G & IQCE & 7 & 2638347 & C & C & C \\
\hline 7_2640985_G_C & IQCE & 7 & 2640985 & $G$ & $G$ & $G$ \\
\hline 7_2647048_C_G & $I Q C E$ & 7 & 2647048 & $\mathrm{C}$ & C & C \\
\hline 7_2691201_C_A & ТTYHЗ & 7 & 2691201 & $A$ & $A$ & $\mathrm{C}$ \\
\hline 7_2699687_-_G & TTYH3 & 7 & 2699687 & - & - & $\mathrm{G}$ \\
\hline 7_2701809_T_C & TTYH3 & 7 & 2701809 & $\mathrm{~T}$ & C & $\mathrm{T}$ \\
\hline 7_2742097_T_C & $A M Z 1$ & 7 & 2742097 & $\mathrm{C}$ & $\mathrm{C}$ & $\mathrm{T}$ \\
\hline 7_2749565_G_A & $A M Z 1$ & 7 & 2749565 & $G$ & $G$ & $G$ \\
\hline 7_2752152_G_A & $A M Z 1$ & 7 & 2752152 & $G$ & $G$ & $G$ \\
\hline 7_2752487_G_A & $A M Z 1$ & 7 & 2752487 & A & $A$ & $G$ \\
\hline 7_2773035_-_AT & GNA12 & 7 & 2773035 & - & - & AT \\
\hline 7_2802173_T_C & GNA12 & 7 & 2802173 & $\mathrm{~T}$ & $\mathrm{~T}$ & $\mathrm{~T}$ \\
\hline 7_2802522_T_C & GNA12 & 7 & 2802522 & $\mathrm{~T}$ & $\mathrm{~T}$ & $\mathrm{~T}$ \\
\hline 7_2834869_C_T & GNA12 & 7 & 2834869 & C & C & $\mathrm{T}$ \\
\hline 7_2854010_G_A & GNA12 & 7 & 2854010 & $G$ & $G$ & $A$ \\
\hline 7_2946461_T_C & CARD11 & 7 & 2946461 & C & C & $\mathrm{C}$ \\
\hline 7_2952912_G_A & $C A R D 11$ & 7 & 2952912 & $\mathrm{G}$ & $\mathrm{G}$ & $G$ \\
\hline 7_2957005_T_C & CARD11 & 7 & 2957005 & C & C & C \\
\hline 7_2958298_C_T & $C A R D 11$ & 7 & 2958298 & C & C & $\mathrm{T}$ \\
\hline 7_2962241_G_A & CARD11 & 7 & 2962241 & $A$ & $A$ & $G$ \\
\hline 7_2962753_G_A & $C A R D 11$ & 7 & 2962753 & $G$ & $G$ & $G$ \\
\hline 7_2966445_T_G & $C A R D 11$ & 7 & 2966445 & $\mathrm{~T}$ & $\mathrm{~T}$ & $\mathrm{~T}$ \\
\hline 7_2966466_GT_- & CARD11 & 7 & 2966466 & GT & GT & GT \\
\hline 7_2968195_G_A & CARD11 & 7 & 2968195 & $G$ & $G$ & $G$ \\
\hline 7_2968361_G_A & CARD11 & 7 & 2968361 & $G$ & $G$ & $G$ \\
\hline 7_2968486_G_C & CARD11 & 7 & 2968486 & $G$ & $G$ & $\mathrm{G}$ \\
\hline 7_2985364_AC_- & $C A R D 11$ & 7 & 2985364 & $A$ & $\mathrm{C}$ & - \\
\hline
\end{tabular}


Supplementary Table S5. Differentially expressed transcripts in A.IV-1 fibroblasts.

\begin{tabular}{|c|c|c|c|c|c|}
\hline Gene name & Ensembl gene id & Description & $\log 2(\mathrm{FC})$ & P-value & Adjusted $p$-value \\
\hline$A B C A 8$ & ENSG00000141338 & $\begin{array}{l}\text { ATP-binding cassette, sub-family A } \\
\text { (ABC1), member } 8\end{array}$ & -4.205829134 & $3.52 \mathrm{E}-05$ & 0.019362186 \\
\hline AC011294.3 & ENSG00000233539 & Uncharacterized protein & 3.130226421 & $2.55 \mathrm{E}-07$ & 0.000427239 \\
\hline ACCS & ENSG00000110455 & $\begin{array}{l}\text { 1-aminocyclopropane-1-carboxylate } \\
\text { synthase homolog (Arabidopsis)(non- } \\
\text { functional) }\end{array}$ & -0.953037549 & $1.28 \mathrm{E}-07$ & 0.00025011 \\
\hline ADAMTSL4 & ENSG00000143382 & ADAMTS-like 4 & -3.153983754 & $5.00 \mathrm{E}-07$ & 0.000732904 \\
\hline$A L D H 1 A 1$ & ENSG00000165092 & $\begin{array}{l}\text { aldehyde dehydrogenase } 1 \text { family, } \\
\text { member } \mathrm{A} 1\end{array}$ & -6.206001924 & $1.99 \mathrm{E}-08$ & $5.40 \mathrm{E}-05$ \\
\hline$A L P L$ & ENSG00000162551 & $\begin{array}{l}\text { alkaline phosphatase, } \\
\text { liver/bone/kidney }\end{array}$ & -3.039649649 & 0.000113858 & 0.038536558 \\
\hline APBA2 & ENSG00000034053 & $\begin{array}{l}\text { amyloid beta (A4) precursor protein- } \\
\text { binding, family A, member } 2\end{array}$ & -2.732442847 & 6.79E-05 & 0.028790694 \\
\hline ARHGAP28 & ENSG00000088756 & Rho GTPase activating protein 28 & 2.800539497 & $1.58 \mathrm{E}-05$ & 0.010684692 \\
\hline C10orf54 & ENSG00000107738 & $\begin{array}{l}\text { chromosome } 10 \text { open reading frame } \\
54\end{array}$ & -1.64124697 & 9.82E-05 & 0.035643913 \\
\hline $\begin{array}{l}\text { C1QTNF9B- } \\
\text { AS1 }\end{array}$ & ENSG00000205861 & C1QTNF9B antisense RNA 1 & -2.047191404 & $3.88 \mathrm{E}-05$ & 0.019780947 \\
\hline$C 2 C D 4 A$ & ENSG00000198535 & $\begin{array}{l}\text { C2 calcium-dependent domain } \\
\text { containing 4A }\end{array}$ & 5.96982663 & 8.95E-07 & 0.001085914 \\
\hline$C 4 A$ & ENSG00000244731 & $\begin{array}{l}\text { complement component 4A (Rodgers } \\
\text { blood group) }\end{array}$ & -6.329532092 & $5.21 \mathrm{E}-11$ & $3.66 \mathrm{E}-07$ \\
\hline$C C D C 180$ & ENSG00000197816 & coiled-coil domain containing 180 & 2.351169896 & 3.81E-05 & 0.019780947 \\
\hline CD200 & ENSG00000091972 & CD200 molecule & -4.053753384 & 0.000135641 & 0.044622222 \\
\hline CHI3L1 & ENSG00000133048 & $\begin{array}{l}\text { chitinase 3-like } 1 \text { (cartilage } \\
\text { glycoprotein-39) }\end{array}$ & 4.852100597 & $6.43 \mathrm{E}-07$ & 0.000837885 \\
\hline CHN1 & ENSG00000128656 & chimerin 1 & -1.617295158 & 2.25E-09 & $1.32 \mathrm{E}-05$ \\
\hline CHRDL2 & ENSG00000054938 & chordin-like 2 & -4.241249176 & 0.000101018 & 0.03591767 \\
\hline CNTN1 & ENSG00000018236 & contactin 1 & -4.135254047 & 8.76E-05 & 0.032879001 \\
\hline COL11A1 & ENSG00000060718 & collagen, type XI, alpha 1 & -4.558619592 & $6.30 \mathrm{E}-05$ & 0.027357715 \\
\hline CSF2RA & ENSG00000198223 & $\begin{array}{l}\text { colony stimulating factor } 2 \text { receptor, } \\
\text { alpha, low-affinity (granulocyte- } \\
\text { macrophage) }\end{array}$ & 3.072895701 & $6.90 \mathrm{E}-05$ & 0.028906369 \\
\hline CYP21A1P & ENSG00000204338 & $\begin{array}{l}\text { cytochrome P450, family } 21 \text {, subfamily } \\
\text { A, polypeptide } 1 \text { pseudogene }\end{array}$ & -4.61429374 & $6.95 \mathrm{E}-06$ & 0.005439088 \\
\hline DUSP2 & ENSG00000158050 & dual specificity phosphatase 2 & 3.527490256 & $1.70 \mathrm{E}-05$ & 0.01128586 \\
\hline ECM1 & ENSG00000143369 & extracellular matrix protein 1 & -1.261675626 & $5.81 \mathrm{E}-07$ & 0.000786019 \\
\hline$E M C N$ & ENSG00000164035 & endomucin & 3.361027658 & 4.95E-05 & 0.0229427 \\
\hline EYA4 & ENSG00000112319 & eyes absent homolog 4 (Drosophila) & -4.200503943 & 4.26E-05 & 0.020550766 \\
\hline FAM21B & ENSG00000152726 & $\begin{array}{l}\text { family with sequence similarity } 21, \\
\text { member B }\end{array}$ & -4.995162913 & 7.93E-08 & 0.000164108 \\
\hline FGF9 & ENSG00000102678 & fibroblast growth factor 9 & 4.555259473 & 5.95E-08 & 0.000133394 \\
\hline FOSB & ENSG00000125740 & $\begin{array}{l}\text { FBJ murine osteosarcoma viral } \\
\text { oncogene homolog B }\end{array}$ & 3.628551087 & $8.80 \mathrm{E}-05$ & 0.032879001 \\
\hline FYB & ENSG00000082074 & FYN binding protein & -4.90648728 & 3.03E-05 & 0.016914087 \\
\hline GRPR & ENSG00000126010 & gastrin-releasing peptide receptor & -4.422707449 & 0.000144048 & 0.046095412 \\
\hline GSTT1 & ENSG00000184674 & glutathione S-transferase theta 1 & -9.843482395 & $6.94 \mathrm{E}-60$ & $2.44 \mathrm{E}-55$ \\
\hline HIVEP3 & ENSG00000127124 & $\begin{array}{l}\text { human immunodeficiency virus type I } \\
\text { enhancer binding protein } 3\end{array}$ & -1.599082154 & $6.64 \mathrm{E}-05$ & 0.028486515 \\
\hline $\mathrm{HOXC10}$ & ENSG00000180818 & homeobox $\mathrm{C} 10$ & -4.942455716 & 2.36E-05 & 0.014065434 \\
\hline HOXC-AS3 & ENSG00000251151 & HOXC cluster antisense RNA 3 & -5.14737488 & 6.01E-05 & 0.026775571 \\
\hline IGF2 & ENSG00000167244 & $\begin{array}{l}\text { insulin-like growth factor } 2 \\
\text { (somatomedin A) }\end{array}$ & -4.063105964 & 3.83E-05 & 0.019780947 \\
\hline IGFBP1 & ENSG00000146678 & $\begin{array}{l}\text { insulin-like growth factor binding } \\
\text { protein } 1\end{array}$ & 2.926653654 & $1.58 \mathrm{E}-05$ & 0.010684692 \\
\hline
\end{tabular}




\begin{tabular}{|c|c|c|c|c|c|}
\hline IGFBP3 & ENSG00000146674 & $\begin{array}{l}\text { insulin-like growth factor binding } \\
\text { protein } 3\end{array}$ & 1.932377253 & $2.71 \mathrm{E}-06$ & 0.002574523 \\
\hline IL17RB & ENSG00000056736 & interleukin 17 receptor B & 3.133016005 & 8.91E-05 & 0.032879001 \\
\hline IL20RA & ENSG00000016402 & interleukin 20 receptor, alpha & 3.104556086 & $3.12 \mathrm{E}-06$ & 0.002749475 \\
\hline IQCE & ENSG00000106012 & IQ motif containing $E$ & -2.198435274 & $1.75 \mathrm{E}-43$ & 3.08E-39 \\
\hline IRF6 & ENSG00000117595 & interferon regulatory factor 6 & -3.931349479 & 7.74E-05 & 0.030946745 \\
\hline $\operatorname{IRX1}$ & ENSG00000170549 & iroquois homeobox 1 & 1.496134127 & $2.70 \mathrm{E}-05$ & 0.015596484 \\
\hline $\operatorname{IRX} 2$ & ENSG00000170561 & iroquois homeobox 2 & 1.161748374 & $4.51 \mathrm{E}-09$ & 1.77E-05 \\
\hline$J A Z F 1-A S 1$ & ENSG00000234336 & JAZF1 antisense RNA 1 & -2.132735824 & 0.000107563 & 0.037119743 \\
\hline$K L K B 1$ & ENSG00000164344 & kallikrein B, plasma (Fletcher factor) 1 & 2.478417669 & 8.84E-05 & 0.032879001 \\
\hline LAMC1 & ENSG00000135862 & laminin, gamma 1 (formerly LAMB2) & -1.201150815 & 2.84E-07 & 0.000453983 \\
\hline LHX9 & ENSG00000143355 & LIM homeobox 9 & 4.298162126 & $1.45 \mathrm{E}-06$ & 0.001650048 \\
\hline LINC00537 & ENSG00000232815 & $\begin{array}{l}\text { long intergenic non-protein coding } \\
\text { RNA } 537\end{array}$ & -3.494641854 & 3.83E-05 & 0.019780947 \\
\hline LINC00578 & ENSG00000228221 & $\begin{array}{l}\text { long intergenic non-protein coding } \\
\text { RNA } 578\end{array}$ & -4.541164267 & 7.46E-09 & 2.39E-05 \\
\hline LINC01048 & ENSG00000230390 & $\begin{array}{l}\text { long intergenic non-protein coding } \\
\text { RNA } 1048\end{array}$ & 2.214309895 & 3.99E-05 & 0.019780947 \\
\hline LINC01117 & ENSG00000224577 & $\begin{array}{l}\text { long intergenic non-protein coding } \\
\text { RNA } 1117\end{array}$ & -2.17227763 & 2.17E-05 & 0.01316806 \\
\hline LRRC16A & ENSG00000079691 & leucine rich repeat containing $16 \mathrm{~A}$ & -1.784218256 & 2.43E-05 & 0.014245821 \\
\hline MAP6 & ENSG00000171533 & microtubule-associated protein 6 & -1.79656788 & $1.30 \mathrm{E}-06$ & 0.001530605 \\
\hline MAP7D2 & ENSG00000184368 & MAP7 domain containing 2 & 2.359306267 & 2.66E-06 & 0.002574523 \\
\hline MBP & ENSG00000197971 & myelin basic protein & -3.402601353 & 7.14E-05 & 0.029220727 \\
\hline MCCC1 & ENSG00000078070 & $\begin{array}{l}\text { methylcrotonoyl-CoA carboxylase } 1 \\
\text { (alpha) }\end{array}$ & -0.554719912 & 0.000128238 & 0.042584679 \\
\hline MECOM & ENSG00000085276 & MDS1 and EVI1 complex locus & -4.511721791 & 3.14E-09 & $1.58 \mathrm{E}-05$ \\
\hline METTL9 & ENSG00000197006 & methyltransferase like 9 & 0.906519962 & $3.86 \mathrm{E}-08$ & $9.70 \mathrm{E}-05$ \\
\hline MFGE8 & ENSG00000140545 & milk fat globule-EGF factor 8 protein & -2.694104724 & $7.44 \mathrm{E}-07$ & 0.000934855 \\
\hline MLLT3 & ENSG00000171843 & $\begin{array}{l}\text { myeloid/lymphoid or mixed-lineage } \\
\text { leukemia (trithorax homolog, } \\
\text { Drosophila); translocated to, } 3 \\
\end{array}$ & 0.969013225 & 2.10E-05 & 0.01316806 \\
\hline MRVI1-AS1 & ENSG00000177112 & MRVI1 antisense RNA 1 & -3.884388307 & 1.04E-05 & 0.007509783 \\
\hline MYO16 & ENSG00000041515 & myosin XVI & -1.962481605 & 0.000117031 & 0.039233207 \\
\hline NDP & ENSG00000124479 & Norrie disease (pseudoglioma) & 3.545635871 & 1.83E-05 & 0.011727901 \\
\hline NR5A2 & ENSG00000116833 & $\begin{array}{l}\text { nuclear receptor subfamily 5, group A, } \\
\text { member } 2\end{array}$ & -4.138425423 & 8.61E-06 & 0.006447287 \\
\hline NTRK3 & ENSG00000140538 & $\begin{array}{l}\text { neurotrophic tyrosine kinase, receptor, } \\
\text { type } 3\end{array}$ & 2.777060262 & 2.95E-05 & 0.016740854 \\
\hline$P K I A$ & ENSG00000171033 & $\begin{array}{l}\text { protein kinase (cAMP-dependent, } \\
\text { catalytic) inhibitor alpha }\end{array}$ & -2.779337899 & $4.26 \mathrm{E}-05$ & 0.020550766 \\
\hline$P N L D C 1$ & ENSG00000146453 & $\begin{array}{l}\text { poly(A)-specific ribonuclease (PARN)- } \\
\text { like domain containing } 1\end{array}$ & 2.95490495 & 2.27E-07 & 0.000399745 \\
\hline PNMA6C & ENSG00000235961 & $\begin{array}{l}\text { paraneoplastic Ma antigen family } \\
\text { member } 6 \mathrm{C}\end{array}$ & -4.033899706 & 0.000151809 & 0.047958078 \\
\hline POMZP3 & ENSG00000146707 & POM121 and ZP3 fusion & -1.717492119 & 3.12E-06 & 0.002749475 \\
\hline POSTN & ENSG00000133110 & periostin, osteoblast specific factor & 2.83484857 & 2.14E-05 & 0.01316806 \\
\hline PRKAR1B & ENSG00000188191 & $\begin{array}{l}\text { protein kinase, cAMP-dependent, } \\
\text { regulatory, type I, beta }\end{array}$ & -1.732349926 & $1.78 \mathrm{E}-05$ & 0.011579369 \\
\hline$P R L R$ & ENSG00000113494 & prolactin receptor & 3.300099077 & 0.000140582 & 0.045398939 \\
\hline PTK2B & ENSG00000120899 & protein tyrosine kinase 2 beta & -1.47679232 & $6.10 \mathrm{E}-05$ & 0.026827045 \\
\hline$R G C C$ & ENSG00000102760 & regulator of cell cycle & -3.93550099 & 7.06E-05 & 0.029220727 \\
\hline$R H B D D 3$ & ENSG00000100263 & rhomboid domain containing 3 & -0.996433377 & $1.05 \mathrm{E}-05$ & 0.007509783 \\
\hline RSPO1 & ENSG00000169218 & R-spondin 1 & -4.313022549 & 5.98E-05 & 0.026775571 \\
\hline S1PR1 & ENSG00000170989 & sphingosine-1-phosphate receptor 1 & -3.093512898 & 5.52E-06 & 0.00452094 \\
\hline
\end{tabular}




\begin{tabular}{|c|c|c|c|c|c|}
\hline SCN1A & ENSG00000144285 & $\begin{array}{l}\text { sodium channel, voltage-gated, type I, } \\
\text { alpha subunit }\end{array}$ & -4.906276861 & $3.80 E-06$ & 0.003184547 \\
\hline SCN9A & ENSG00000169432 & $\begin{array}{l}\text { sodium channel, voltage-gated, type } \\
\text { IX, alpha subunit }\end{array}$ & -3.142530704 & $1.83 E-06$ & 0.001898572 \\
\hline$S D K 1$ & ENSG00000146555 & sidekick cell adhesion molecule 1 & 3.188897352 & 4.55E-05 & 0.021372366 \\
\hline SFRP1 & ENSG00000104332 & secreted frizzled-related protein 1 & -4.10661345 & $5.21 \mathrm{E}-07$ & 0.000732904 \\
\hline$S H E$ & ENSG00000169291 & Src homology 2 domain containing $E$ & 3.455259022 & 1.63E-06 & 0.001733572 \\
\hline$S L A$ & ENSG00000155926 & Src-like-adaptor & -2.701260288 & 3.99E-05 & 0.019780947 \\
\hline SLC16A11 & ENSG00000174326 & solute carrier family 16 , member 11 & -2.677392243 & 4.37E-05 & 0.020765473 \\
\hline SLC30A8 & ENSG00000164756 & $\begin{array}{l}\text { solute carrier family } 30 \text { (zinc } \\
\text { transporter), member } 8\end{array}$ & 6.104148946 & $1.35 \mathrm{E}-07$ & 0.000250798 \\
\hline SLC35D3 & ENSG00000182747 & solute carrier family 35, member D3 & 9.179494017 & $2.46 \mathrm{E}-31$ & 2.89E-27 \\
\hline$S L C 4 A 3$ & ENSG00000114923 & $\begin{array}{l}\text { solute carrier family } 4 \text { (anion } \\
\text { exchanger), member } 3\end{array}$ & -2.366325383 & $7.28 \mathrm{E}-05$ & 0.029469479 \\
\hline SMOC1 & ENSG00000198732 & $\begin{array}{l}\text { SPARC related modular calcium } \\
\text { binding } 1\end{array}$ & -5.03813069 & 0.000104067 & 0.036268803 \\
\hline SPON2 & ENSG00000159674 & spondin 2 , extracellular matrix protein & -2.639592845 & 0.000112611 & 0.038484373 \\
\hline$S R D 5 A 2$ & ENSG00000049319 & $\begin{array}{l}\text { steroid-5-alpha-reductase, alpha } \\
\text { polypeptide } 2 \text { (3-oxo-5 alpha-steroid } \\
\text { delta 4-dehydrogenase alpha 2) } \\
\end{array}$ & 4.448074003 & 8.97E-05 & 0.032879001 \\
\hline STAG3L2 & ENSG00000160828 & stromal antigen 3-like 2 (pseudogene) & -0.881204831 & $9.94 \mathrm{E}-05$ & 0.035716372 \\
\hline TCF21 & ENSG00000118526 & transcription factor 21 & -6.04609974 & $1.52 E-06$ & 0.001676294 \\
\hline ULK4 & ENSG00000168038 & unc-51 like kinase 4 & 1.147447551 & 6.46E-09 & $2.28 \mathrm{E}-05$ \\
\hline WASF3 & ENSG00000132970 & WAS protein family, member 3 & 0.972046987 & $2.81 \mathrm{E}-06$ & 0.002603617 \\
\hline$X K R 9$ & ENSG00000221947 & $\begin{array}{l}\text { XK, Kell blood group complex subunit- } \\
\text { related family, member } 9\end{array}$ & -1.989973823 & 0.000159916 & 0.049814665 \\
\hline ZBTB16 & ENSG00000109906 & $\begin{array}{l}\text { zinc finger and BTB domain containing } \\
16\end{array}$ & -4.844890791 & 7.83E-05 & 0.030946745 \\
\hline ZFPM2 & ENSG00000169946 & $\begin{array}{l}\text { zinc finger protein, FOG family } \\
\text { member } 2\end{array}$ & -3.60011069 & $5.25 \mathrm{E}-14$ & $4.62 \mathrm{E}-10$ \\
\hline ZNF804A & ENSG00000170396 & zinc finger protein $804 \mathrm{~A}$ & -5.483825868 & 4.34E-09 & 1.77E-05 \\
\hline
\end{tabular}


Supplementary Table S6. GO enrichment analysis. Show the distribution of GO terms exhibiting statistical significant differences of mis-regulated transcripts in A.IV-1 fibroblast using WebGestalt 2019 (Wang, Vasaikar, Shi, Greer, \& Zhang, 2017).

\begin{tabular}{|c|c|c|c|c|c|c|c|c|c|}
\hline GO terms & Description & $\begin{array}{c}\text { Gene } \\
\text { set size }\end{array}$ & Overlap & Expected Value & Enrichment Ratio & pValue & FDR & overlap_ld & user_Id \\
\hline GO:0009888 & tissue development & 1613 & 24 & 7.625171279802685 & 3.1474702822178497 & $1.528766712111107 \mathrm{e}-7$ & 0.0012670418509976855 & 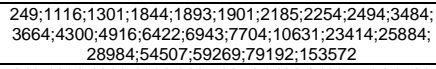 & 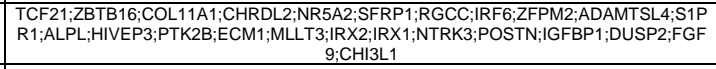 \\
\hline GO:0001503 & ossification & 339 & 11 & 1.6025623458481775 & 6.864007524261469 & $4.940246167706164 \mathrm{e}-7$ & 0.0020472380118974343 & $249 ; 1301 ; 1893 ; 1901 ; 2185 ; 22294 ; 3486 ; 6422 ; 7704 ; 2588$ & SMOC1;ZBTB16;COL11A1;CHRDL2;FFRP1;;1PR1;ALLPL;PTK2B;ECM1;/GFBP3;FGF9 \\
\hline GO:0030038 & $\begin{array}{l}\text { intractile aci } \\
\text { as }\end{array}$ & 76 & 5 & 0.35927651411345574 & 13.916857360793287 & $2.9031950272262463 \mathrm{e}-5$ & 0.060154200964127824 & $1901 ; 2185 ; 6422 ; 28984 ; 55604$ & SFRP1;RGCC;S1PR1;CARMLL1;PTK2B \\
\hline GO:0043149 & stress fiber & 76 & 5 & 0.35927651411345574 & 13.916857360793287 & $2.9031950272262463 \mathrm{e}-5$ & 0.060154200964127824 & $1901 ; 2185 ; 6422 ; ; 28984 ; 555604$ & SFRP1:RGCC;:S1PR1;CARMIL1;PTK2B \\
\hline GO:0048584 & $\begin{array}{l}\text { positive regulation of response to } \\
\text { stimulus }\end{array}$ & 1768 & 21 & 8.35790627569197 & 2.512590989573087 & 4. $138463705938289 e-5$ & 0.06101540527461857 & $\begin{array}{l}1116 ; 1123 ; 1893 ; 1901 ; 2070 ; 2185 ; 2254 ; 25333 ; 3486 ; 381 \\
8 ; 4300 ; 4916 ; 5618 ; 6422 ; 6503 ; 10417 ; ; 28984 ; 53832 ; 555 \\
40 ; 126669 ; 284654\end{array}$ & 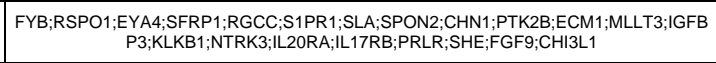 \\
\hline & & & 3 & 0.0709098 & & & 0.06101540527461857 & & \\
\hline & & & 6 & 0.7989175116470265 & 7.5101620787239525 & & 0.12546232977013955 & & $\begin{array}{l}\frac{1 ; Z Z B T B 16 ; S F R P 1 ;}{2} \\
\text { RGCC;S1PR1;PTK2 }\end{array}$ \\
\hline $\begin{array}{l}\text { GO:0048646 } \\
\text { GO:0001501 }\end{array}$ & & 876 & 13 & 4.14113455741299 & 3.1392363179141025 & $2.015277408380367 \mathrm{e}-4$ & 0.12546232977013955 & 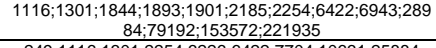 & 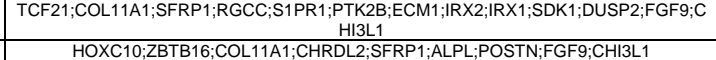 \\
\hline GO:0001501 & & 439 & 9 & 2.075294601260619 & 4.33673368327226 & $2.1232290176698143 \mathrm{e}-4$ & 0.12546232977013955 & $249 ; 1116 ; 1301 ; 2254 ; 3226 ; 6$ & HOXC10;ZBTB16;COL11A1;CHRDL2;SFRP1;ALLL;:POSTN;FGF9;CHIIL1 \\
\hline GO:0072047 & $\begin{array}{l}\text { proximalldistal pattern formation } \\
\text { involved in nephron develoment }\end{array}$ & 5 & 2 & .023636612770622088 & 84.61449275362318 & $2.182366244216638 \mathrm{e}-4$ & 0.12546232977013955 & 79192;153572 & \\
\hline
\end{tabular}




\section{Supplementary Table S7. Summary of transcriptome mapping results.}

\begin{tabular}{|c|c|c|c|c|c|}
\hline Sample ID & \#total reads & \#aligned reads & $\begin{array}{c}\text { \% aligned } \\
\text { reads }\end{array}$ & $\begin{array}{c}\text { \#uniquely } \\
\text { mapped }\end{array}$ & $\begin{array}{c}\text { \% uniquely } \\
\text { aligned reads }\end{array}$ \\
\hline A.IV-1.1 & $306,265,974$ & $303,190,132$ & 99.00 & $293,189,972$ & 96.70 \\
\hline A.IV-1.2 & $344,782,588$ & $341,370,324$ & 99.01 & $330,182,914$ & 96.72 \\
\hline ARN1.1 & $338,129,498$ & $335,557,854$ & 99.24 & $325,618,112$ & 97.04 \\
\hline ARN1.2 & $301,339,526$ & $298,096,006$ & 98.92 & $289,521,446$ & 97.12 \\
\hline ARN2.1 & $311,917,536$ & $307,641,094$ & 98.63 & $296,837,798$ & 96.49 \\
\hline ARN2.2 & $336,751,354$ & $333,728,602$ & 99.10 & $322,903,810$ & 96.76 \\
\hline ARN3.1 & $403,718,870$ & $400,433,942$ & 99.19 & $383,740,470$ & 95.83 \\
\hline ARN3.2 & $299,879,030$ & $296,763,108$ & 98.96 & $286,203,188$ & 96.44 \\
\hline ARN4.1 & $318,588,494$ & $315,510,058$ & 99.03 & $304,438,002$ & 96.49 \\
\hline ARN4.2 & $320,107,070$ & $316,729,460$ & 98.94 & $304,559,776$ & 96.16 \\
\hline ARN5.1 & $363,584,984$ & $360,496,138$ & 99.15 & $349,081,194$ & 96.83 \\
\hline ARN6.1 & $324,984,406$ & $321,703,444$ & 98.99 & $311,631,678$ & 96.87 \\
\hline ARN6.2 & $364,763,088$ & $361,575,516$ & 99.13 & $349,853,468$ & 96.76 \\
\hline
\end{tabular}




\section{SUPPLEMENTARY REFERENCES}

Kim, D., Pertea, G., Trapnell, C., Pimentel, H., Kelley, R., \& Salzberg, S. L. (2013). TopHat2: accurate alignment of transcriptomes in the presence of insertions, deletions and gene fusions. Genome Biol, 14(4), R36. doi:10.1186/gb-2013-14-4-r36

Langmead, B., Trapnell, C., Pop, M., \& Salzberg, S. L. (2009). Ultrafast and memory-efficient alignment of short DNA sequences to the human genome. Genome Biol, 10(3), R25. doi:10.1186/gb2009-10-3-r25

Nevers, Y., Prasad, M. K., Poidevin, L., Chennen, K., Allot, A., Kress, A., . . Lecompte, O. (2017). Insights into Ciliary Genes and Evolution from Multi-Level Phylogenetic Profiling. Mol Biol Evol, 34(8), 2016-2034. doi:10.1093/molbev/msx146

Wang, J., Vasaikar, S., Shi, Z., Greer, M., \& Zhang, B. (2017). WebGestalt 2017: a more comprehensive, powerful, flexible and interactive gene set enrichment analysis toolkit. Nucleic Acids Research, 45(W1), W130-W137. doi:10.1093/nar/gkx356 

Input
Status
NM_152558.4:c.895_904del OK
NM_003322.5:c.1198C >T OK
NM_152558.4:c.895_904del OK
NM_152558.4:c.1350_1353del OK
NM_001692.3:c.175-1G>C OK 